\title{
Minimal models for lipid membranes: local modifications around fusion objects
}

\section{Dissertation}

\author{
zur Erlangung des Doktorgrades
}

der Mathematisch-Naturwissenschaftlichen Fakultäten

der Georg-August-Universität zu Göttingen

vorgelegt von

\section{Giovanni Marelli}

aus Desenzano del Garda, Italien

Göttingen 2012 
D 7

Referent: Prof. Dr. Marcus Müller Koreferent: Prof. Dr. Bert de Groot

Tag der mündlichen Prüfung: 


\section{Contents}

$\begin{array}{lll}1 & \text { model } & 7\end{array}$

1.1 efficient modelling of collective phenomena . . . . . . . . . . . . 7

1.2 solvent-free model $\ldots \ldots \ldots \ldots \ldots \ldots$

1.3 dissipative particle dynamics $\ldots \ldots \ldots$. . . . . . . . . . . . . 12

1.4 homopolymer melt . . . . . . . . . . . . . . . . . . . . . . . . 15

1.5 bilayer membranes . . . . . . . . . . . . . . . . . . . . 17

2 setting

$\begin{array}{lr}\text { the Monte Carlo simulations } & 19\end{array}$

2.1 lipid reservoir . . . . . . . . . . . . . . . . . . . . . . . . . . . . . . 19

2.2 grand canonical Monte Carlo for lipid chains . . . . . . . . . . . . 19

2.3 configurational bias: insertion $\ldots \ldots \ldots \ldots \ldots . \ldots \ldots \ldots$

2.4 configurational bias: deletion . . . . . . . . . . . . . . . . . . 22

2.5 configurational bias: acceptance criterion . . . . . . . . . . . . 23

2.6 added energy $\ldots \ldots \ldots \ldots \ldots \ldots \ldots \ldots$

2.7 chemical potential of the molecules . . . . . . . . . . . . . . . . 24

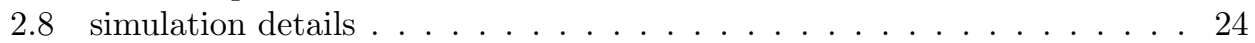

2.9 test runs for Monte Carlo simulations . . . . . . . . . . . . . 26

2.9 .1 lipids in a bilayer . . . . . . . . . . . . . . . 27

$\begin{array}{lll}3 & \text { inclusion stability } & 29\end{array}$

3.1 energetic stability of a hydrophobic inclusion in bilayer membranes . . . 29

3.2 model $\ldots \ldots \ldots \ldots \ldots$

3.3 simulation details $\ldots \ldots \ldots \ldots \ldots \ldots$. . . . . . . . . . . . . . . . . 32

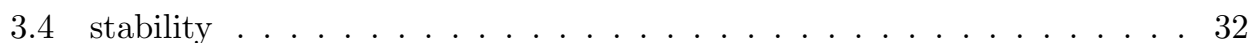

3.5 chemical potential of the nanoparticle . . . . . . . . . . . . . . 33

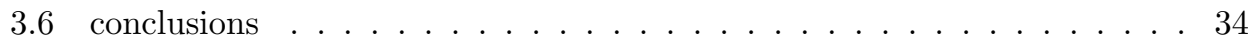

4 mechanical and phase properties of planar membranes 35

4.1 lipid composition . . . . . . . . . . . . . . . . 36

4.2 exploring the phase space $\ldots \ldots \ldots \ldots \ldots$

4.3 thickness $\ldots \ldots \ldots \ldots \ldots \ldots$

4.4 time scale . . . . . . . . . . . . . . . . . . . . . . . . 37

4.5 thickness, lateral area, and density fluctuations . . . . . . . . . . . . 39

4.6 energetic contributions $\ldots \ldots \ldots \ldots$. . . . . . . . . . . . . 40

4.7 tables $\ldots \ldots \ldots \ldots \ldots \ldots$. . . . . . . . . . . . . . . . . . . . 44

4.8 modeling of an experiment . . . . . . . . . . . . . . . . . 45

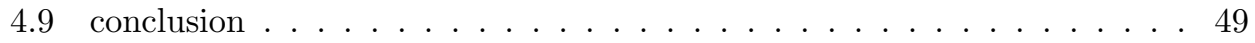

5 stalk dynamics

and morphology 51

5.1 introduction $\ldots \ldots \ldots \ldots \ldots \ldots \ldots$. . . . . . . . . . . . . . 51

5.2 Martini simulations . . . . . . . . . . . . . . . . . . . . . . 52

5.3 simulation details $\ldots \ldots \ldots \ldots \ldots \ldots$. . . . . . . . . . . . . . 53 
5.4 building a stalk . . . . . . . . . . . . . . . . . . 53

5.5 shape reconstruction of a linear stalk . . . . . . . . . . . . . . 54

5.6 position and size of a circular stalk . . . . . . . . . . . . . 55

5.7 line tension of the stalk $\ldots \ldots \ldots \ldots \ldots$

5.8 rigidity of the stalk $\ldots \ldots \ldots \ldots \ldots \ldots \ldots$

5.9 density profile $\ldots \ldots \ldots \ldots \ldots \ldots \ldots$

5.10 stalk elongation . . . . . . . . . . . . . . . . . . . . . . . 59

5.11 bilayer repulsion $\ldots \ldots \ldots$. . . . . . . . . . . . . . . . . . 60

5.12 conclusions $\ldots \ldots \ldots \ldots$. . . . . . . . . . . . . . 62

6 inclusion interactions $\quad 65$

6.1 minimal models for thinning of lipid membranes by transmembrane pro-

6.2 soft description . . . . . . . . . . . . . . . . . . . . 66

6.3 parameter space $\ldots \ldots \ldots \ldots$. . . . . . . . . . . . . . . . . 6 67

6.4 continuum model . . . . . . . . . . . . . . . . . . . . . . 67

6.5 simulation description $\ldots \ldots \ldots \ldots$. . . . . . . . . . . . 70

6.6 macroscopic scale . . . . . . . . . . . . . . . . . . . . . . 70

6.7 thickness characteristics $\ldots \ldots \ldots \ldots \ldots$. . . . . . . . . . . . . .

6.8 superposition of effects $\ldots \ldots \ldots \ldots \ldots \ldots$

6.9 multiple inclusions $\ldots \ldots \ldots \ldots$. . . . . . . . . . . . . . 73

6.10 microscopic scale . . . . . . . . . . . . . . . . . . . . . . . . . 74

6.11 conclusion . . . . . . . . . . . . . . . . . . . 76

\begin{tabular}{|ll|}
\hline $\mathbf{7}$ & line tension of the pore in presence \\
\hline of defectants & $\mathbf{7 7}$
\end{tabular}

7.1 pore-protein interactions . . . . . . . . . . . . . . . . . . . 77

7.2 center, area and acylindricity of the pore $\ldots \ldots \ldots \ldots 78$

7.3 thickness profile between nanoparticle and pore . . . . . . . . . . . . 80

7.4 line tension of the pore $\ldots \ldots \ldots \ldots$. . . . . . . . . . 80

7.5 partitioning of oil $\ldots \ldots \ldots \ldots \ldots$. . . . . . . . . . . . . 81

7.6 bond length $\ldots \ldots \ldots \ldots \ldots$. . . . . . . . . . . . . . . . . . . 82

7.7 lateral pressure profile $\ldots \ldots \ldots \ldots$. . . . . . . . . . . . . 84

8.8 conclusion $\ldots \ldots \ldots \ldots \ldots$

$\begin{array}{llr}8 \text { conclusions and outlook } & 87\end{array}$

8.1 summary . . . . . . . . . . . . . . . . . . . . 87

8.2 conclusions $\ldots \ldots \ldots \ldots$

8.3 outlook $\ldots \ldots \ldots \ldots \ldots \ldots$

\begin{tabular}{lr}
\hline appendix & 91
\end{tabular}

8.4 discrete solution of the continuum model . . . . . . . . . . . . . . . . 91

8.5 interpolation $/$ smoothing $\ldots \ldots \ldots \ldots$. . . . . . . . . . . . . . . . . . . . . . . . . . .

8.6 domain decomposition $\ldots \ldots \ldots \ldots$. . . . . . . . . . . . . . 94 


\section{introduction}

\section{functionality and structure of lipid membranes}

The functionality of cells and organelles is assured by the separation of the cytosol from the extracellular liquid Luckey (2008)]. The intracellular and extracellular liquids differ in their DNA/RNA, protein and ion content. The lipid membrane is the envelope that defines the boundary of the cell and prevents the mixing of liquid contents.

The exchange between the two liquids, like the transport of drugs and compounds, and changes in membrane topology (for example fusion and fission), involve a reordering of the lipids inside the membrane [Luckey (2008)]. The structure of the membrane depends on its lipid composition, temperature and external pressure. In membranes lipids are ordered into planar lamellar bilayers, they align parallel to each other and the polar head groups face the solvent and shield the apolar tails from unfavorable interactions with the solvent Luckey (2008)].

The membrane hence consists of two coupled leaflets of lipids, with interesting mechanical properties Helfrich (1985); Peliti and Leibler (1985); Discher et al. (1998); Fygenson and Libchaber (1997)]. A lipid membrane is about $4-5[\mathrm{~nm}]$ thick and extends in the lateral dimension over much larger orders of magnitude $\left(10-100\left[\mu \mathrm{m}^{2}\right]\right.$ in the case of giant unilamellar vesicles, GUV). On the macroscopic scale it can be described as a continuum elastic sheet, where the shape fluctuation is controlled by the bending and stretchability of the metric of a 2d surface [Seifert et al. (1991); Miao et al. (1991, 1994)].

The elastic properties of the membrane and its transport properties (e.g. permeability) is dictated by the lipid structure on the molecular scale.

\section{collective processes in lipid membrane}

The stabilisation of curved local structure and pores, the opening of membrane channels, the stages of membrane fusion such as the formation of a stalk (a lipid bridge between two opposing membranes), and membrane fission are processes that require local reordering of many lipids and are often driven by transmembrane or surface proteins Huang (1986b); Fattal and Ben-Shaul (1993); Helfrich and Jakobsson (1990a); Partenskii and Jordan (2002); Nielsen et al. (1998); Marčelja (1976)]. These proteins induce a local stress on the lipids around them and alter their equilibrium conformation, driving the lipids to a new transient or stable conformation. Stalk and pore formations are important steps for lowering the energetic barrier to membrane fusion Katsov et al. (2006); Schick et al. (2005); Risselada et al. (2012)]. The discovery and classification of fusion pathways highlights important features of the cell cycle. Fusion between cells is essential for synaptic transmission and viral infection, and the role of proteins in this process is a matter of debate.

\section{fusion processes}

The fusion process between two bilayers is a complex change in the topological structure of the bilayers that requires intermediate steps. The fundamental steps are the lipid mixing between the two membranes (stalk) and, possibly, the formation of a pore 
to allow the exchange between the internal and external liquids (see fig.1). There exists many different possibilities about the sequence of these steps and the pathways of the fusion process. One possibility considers the formation of the stalk as first, the expansion of this stalk in a hemifusion diaphragm, and the formation of a fusion pore in the hemi-fusion diaphragm [Jahn and Grubmüller (2002); Kozlovsky et al. (2004)]. An alternative pathway considers that after the formation of the stalk a pore is created close to the ends of the stalk and the stalk elongates around the pore. When the stalk has encircled the pore the fusion pore is created on the opposed bilayer Katsov et al. (2006); Schick et al. (2005); Müller et al. (2003)]. Alternatively the pore can be originated first by peptides and lowers the energy barrier for the formation of a stalk. At that point the stalk elongates around the pore and follows the terminal part of the pathway described above Risselada et al. (2012)]. These pathways are triggered by the presence of proteins embedded in the membrane and local tensions. The fusion objects (peptides, pores and stalks see fig 1) locally modify the membrane and interact by a long range perturbation of the lipid conformations.

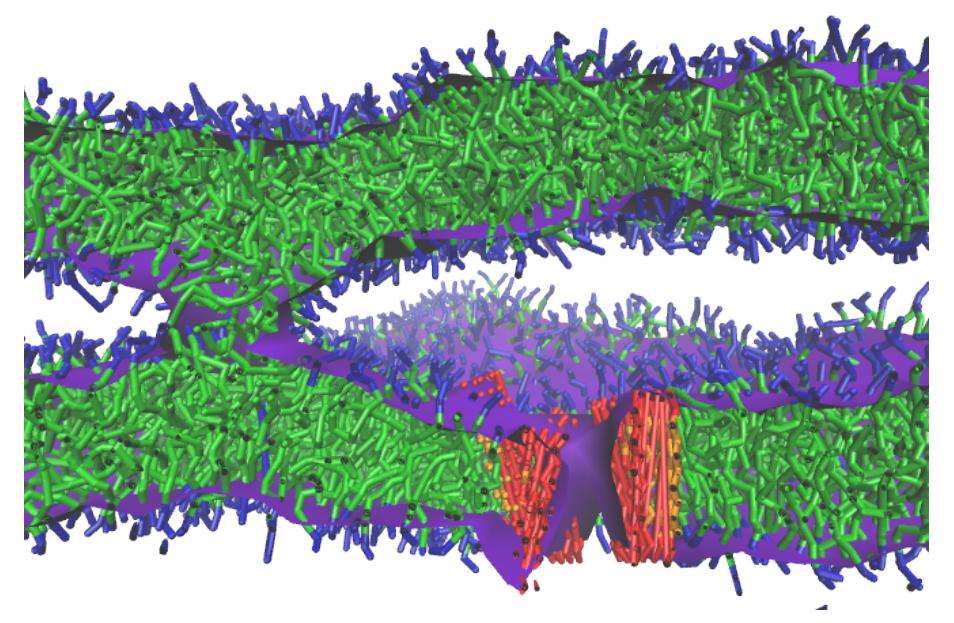

FIGURE 1: Cooperative behaviour between fusion objects. In the picture a pore is stabilized by transmembrane peptides and interacts with a stalk via the deformation of the membrane nearby. The bonds describing the peptides are colored in red and the violet surface corresponds to the isosurface of the hydrophobic density.

Modifications between neighboring objects interact via changes of the lipid bilayers whose effect is crucial in steering membrane fusion. Local modifications of membrane structure cause packing frustration of the lipids that are constrained to sit parallel to each other in the membrane. Short hydrophobic chains, like oil, embedded in the membrane can relax the frustration of the lipid chains and partition where most of this frustration is present.

\section{solvent-free coarse-grained simulations}

Fusion process pathways are far from the possibility of experimental observation due to spatial and temporal resolution. The start of their evolution involves only a handful of lipids over a time scale of about a nanosecond. The structural changes themselves, however, can involve a large number of lipids and require the study of the evolution of the system over many $[\mu s]$.The span of time and length scales makes coarse-grained models necessary and these models are well suited for fusion because there is universal behaviour (i.e. system with different interactions exhibit similar collective behaviour). Coarse-grained models are widely used in the physics of membranes VVenturoli et al. (2006); Müller et al. (2006)] and the most relevant applications of those models are : 
- mechanical properties of planar membrane: Brandt et al. (2011); Waheed and Edholm (2009); Shinoda and Okazi (1998); Neder et al. (2010); Zemel et al. (2008); Gao et al. (2007); Loison et al. (2003)],

- lipid diffusion: Javanainen et al. (2013); Apajalahti et al. (2010a); Klauda et al. (2006); Cooke and Deserno (2005)],

- lipid-protein interactions: May (2002); West et al. (2009); Branningan and Brown (2007, 2006); Niemelä et al. (2010); Venturoli et al. (2005); Reister and Seifert (2005); Sintes and Baumgärtner (1997); Schmidt et al. (2008); Fattal and Ben-Shaul (1993); Marčelja (1976)],

- stalk formation: Marrink and Mark (2003); Smirnova et al. (2010); Markvoort and Marrink (2011); Müller et al. (2012)],

- pore formation: Müller and Schick (1996); Wang and Frenkel (2005); Tolpekina et al. (2004); Rzepiela et al. (2009)],

- membrane fusion: Katsov et al. (2006); Schick et al. (2005); Müller et al. (2003); Risselada and Grubmüller (2012); Shillcock and Lipowsky (2007); Grafmüller et al. (2009); Risselada et al. (2012)],

- membrane fission: Yang and Ma (2012)].

\section{modeling}

Among all the different models present in the literature, we choose to construct a soft, solvent-free coarse-grained model that captures the essential properties of the membrane shape evolution that involve a large number of lipids, around 10000-100000 chains. The essential property of lipids is their amphiphilic nature (polar head groups and apolar tails), so we reduce the complexity of real lipids by dividing the chains into polar and apolar blocks Daoulas and Müller (2010); Hömberg and Müller (2010)]. The removal of solvent particles from the simulation solves the problem of equilibrating big structures under topology changes and reduces by a substantial fraction the number of interactions to be calculated. The removal of solvent molecules is performed comprising their contribution by rescaling the interactions of the polar and apolar beads. The rescaled interactions are derived from a density functional Hamiltonian that represents a third-order virial expansion in term of local densities. This expansion is the lowest-order approximation for a phase separation between liquid and vapor phases, and, by setting the virial coefficients properly, we can represent a hydrophobic chainmelt in good or bad solvent conditions. More precisely, the virial coefficients set the compressibility and the density of the melt and the incompatibility between the two species. The interactions are coarse-grained in the sense that we lump the interaction of many single atoms into one bead and we rescale the virial coefficients to be invariant with respect to the discretization of the molecular contour. The interactions are soft because there is no harsh excluded volume interactions. This is characteristic for coarse-grained models where an effective interaction center represents a group of atoms because the center of mass of these collection of atoms can overlap even if the atoms cannot. All the forces in the system (conservative, dissipative and random) are smoothed by weighting functions to improve their integrability (improved numerical stability and larger time steps) and the energy sampling (narrower distribution of the interaction values) Groot and Warren (1998); Trominov et al. (2002)]. The sampling of energetic contributions via thermodynamic fluctuations allows exploration of the configuration space to be achieved in a limited amount of time, avoiding the use of more advanced techniques (e.g. umbrella sampling) [Plischke and Bergensen (1994)]. 


\section{structure of the thesis}

This work is divided into seven chapters. The first two chapters are dedicated to the presentation of our model and simulation techniques. In the third chapter the calculation of the chemical potential of a hydrophobic inclusion inside the membrane is presented. In the fourth chapter, the mechanical properties of bilayer membranes are analyzed, for the parametrization of the continuum model, and to obtain important reference values. In the fifth chapter are discussed the static and dynamic properties of stalks. The sixth chapter studies the local modifications around a transmembrane protein and the predictions of the continuum model. In the last chapter we show how the line tension of pore changes in the presence of defectants and how oil partitions around fusion objects.

\section{[c III] inclusion stability}

In the third chapter, we model a hydrophobic inclusion as a spherical Lennard-Jones potential and explore the parameter space (the inclusion radius and surface energy) to isolate the parameters that assure stability inside the membrane. The presence of the inclusion modifies the surrounding membrane, and the embedding of the inclusion may not be energetically favourable, resulting in ejection of the nanoparticle and some coating lipids from the membrane. Therefore we calculate the chemical potential of the inclusion inside the membrane to compare it with the chemical potential of a nanoparticle with a lipid coating using thermodynamic integration in the grand canonical ensemble.

\section{[c IV] planar lamellar membranes}

Once we have set up the simulations, we study the influence of our model's parameters on the macroscopic quantities of the self-assembled structures. The search of the parameter range for fluid planar membranes proceeds in the following systematic order. First we study the influence of chain architecture on the self-assembled phases and identify the lipid architectures that self-assemble into inverted micelle, inverted hexagonal, rhomboidal and lamellar phase. Among the lipids corresponding to the lamellar phase, we study succesively the influence of model's parameters on the bending rigidity and area compressibility, and isolate the range where the membrane is in a stable fluid phase. The incompressibility parameter also controls the diffusivity of lipids and we calculate the different diffusion constants for different phases. For a fixed compressibility we use the hydrophobic thickness to set the length scale of the model.

\section{[c V] stalk morphology and evolution}

In this chapter we analyse the mechanical and dynamic properties of a stalk. The stalk is a lipid bridge between two opposed membranes, which has a similar shapes but different activation barrier depending on the lipid composition. We identify two lipids architectures that we call PE-like and PC-like lipids. The first architecture gives rise to linearly elongated stalks, the second forms circular ones. We develop a method to reconstruct the linear and the circular shape of the stalk, and we trace its position and shape deformation. We quantify how the contribution of the lateral tension and dehydration influences bilayer repulsion. Finally, we compare the shape of the stalk with different models and experiments and point out universal behaviours.

\section{[c VI] thickness profile around transmembrane proteins}

After having studied the bulk properties of planar membranes, we investigate how a transmembrane cylindrical hydrophobic inclusion (i.e. a coarse-grained representation of a protein) modifies the surrounding membrane. The local modification of the lipids 
around the protein can cause the stabilisation of a pore, the addition of local stress, and the lowering of the activation energy for the formation of a stalk. Starting works studying on lipid modification around a single protein in coarse-grained models Venturoli et al. (2006)], we want to extend this research to the effect of a cluster of proteins. We also test the ability of the continuum model to predict the behaviour observed in our simulations. The continuum model allows for a finer exploration of the parameter space (interficial energy, hydrophobic mismatch and radius of the single protein). We study the superposition of membrane deformation for a cluster of proteins and we find the minimal configuration for the stabilisation of a toroidal pore.

\section{[c VII] line tension of pores in the presence of defectants}

The presence of one protein lowers the energy barrier to formation of a pore, and we quantify this effect by calculating the line tension of the pore with and without external peptides. If we add short hydrophobic chains (oil) inside the bilayer, we observe an increase in the line tension of the pore and the mean separation between the pore and the protein. To study the effect of the oil, we calculate the partitioning of oil between the protein and the pore. More detailed information about the stress release due to oil is done by calculating the stress profile around the inclusion and the pore. The presence of oil modifies the thickness profile around the two objects and we calculate the pore peptide distance with and without oil. 


\section{Chapter 1}

\section{model}

\section{1 efficient modelling of collective phenomena}

In this chapter we develop a minimalistic model to study collective phenomena of many lipids in bilayer membranes. The model we use includes an implicit solvent and reduces the complexity of the molecular architecture of lipids into diblock chains, where the atomistic description is substituted with a polar/apolar duality. We use soft and coarse-grained interactions where each of the beads represents a collection of atoms and interacts with its neighbours by effective, mesoscopic interactions. These interactions should take into account the effect of the missing water molecule and represent the phase separation between a melt of apolar chains and the polar solvent. The coarse-graining procedure and the lack of solvent requires the addition of a thermostat. To accurately calculate membrane fluctuations and large scale phenomena involving ten thousands of chains, we have to assure correct conservation of the momentum. The dissipative particle dynamics thermostat (DPD) Pagonabarraga and Frenkel (2001); Espanol and Warren (1995); Groot and Warren (1998)] improves the Langevin thermostat in the sense that it conserves locally momentum, which is essential for large scale hydrodynamics. Another important feature of the thermostat is the softness of the forces, which facilitates stable integration of the equation of motion. The integration of the equation of motion is done with the velocity Verlet algorithm. Once the simulation program was written, we explored its parameter space for the self-assembly of lipids into planar fluid membranes. After the formation of a stable bilayer, we introduce a description of a protein or a nanoparticle that interacts with both types of beads. It is repulsive towards the hydrophilic beads and attractive towards the hydrophobic ones, save for hard core repulsion.

The properties of the simulation scheme are briefly introduced in the following sections.

\section{2 solvent-free model}

equation of state

An equation of state can be formulated as a virial expansion in powers of density where every virial coefficient represents a $n$-body interaction Hömberg and Müller (2010)].

$$
P=k_{B} T \sum_{n=1} c_{n} \rho^{n}
$$

The first order term, $c_{1}=1$ represents an ideal gas where no interaction occurs between the particles. Further terms are required when pairwise interactions, $c_{2} \neq 0$, or multibody interactions, $c_{n>2} \neq 0$, occur. Homopolymer chains in a polar solvent aggregate with each other and form a melt that minimizes the unfavourable contact with the 
solvent. The system is nearly incompressible and the melt density fluctuates around the coexistence value where the liquid phase coexists with the vapor phase with negligible density (i.e. the solvent free model). Since we have removed the solvent, the pressure of the whole system is zero as the polymer melt coexists with a vapour of vanishingly low density (basically vacuum) and, at equilibrium, the pressure normal to the bilayer must be the same at every point in the space.

The equation of state, $P(\rho)$, that describes the coexistence of the homopolymer melt in a fluid phase with its vapor is at the lowest-order a third degree polynomial, i.e. the lowest order curve that crosses the point $(0,0)$ and $\left(0, \rho_{\text {coex }}\right)$. For densities close to the density coexistence (miscibility gap), phase separation occurs and the system is spatially inhomogeneous.

Figure 1.1: Mean-field Equation of state of the liquid-vapour interface. The curve represent the third-order polynomial expansion of the equation of state, the tangent shows the derivative of the equation of state with respect to the density, i.e. the reciprocal of the compressibility of the system

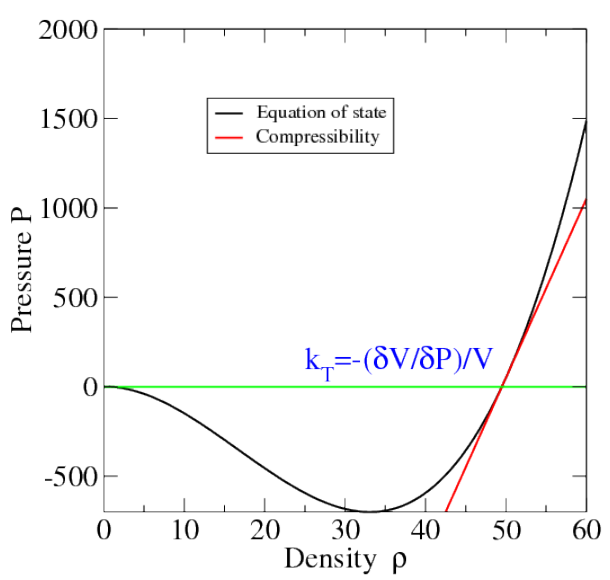

In the graph, (see fig 1.1 , we show the line represented by the equation

$$
\frac{P}{k_{B} T}=\rho+c_{2} \rho^{2}+c_{3} \rho^{3}
$$

where the coexistence density is at the point where the line crosses the abscissa and can refer to the density of hydrophobic beads, $\rho_{A}$, or the density of chains $\rho_{c}$. The slope of the derivative of the equation of state at the coexistence density shown in the graph marks the inverse of the compressibility of the system. The coexistence density of the hydrophobic melt and the thickness of the bilayer membrane in the tensionless state dictate the areal density of amphiphiles in a bilayer membrane. This is a key characteristics of the membrane and values for different system are available in the literature [Bermudez et al. (2002)], Bermudez et al. (2004)] with values around $\rho_{\text {coex }}=10-100\left[R_{e}^{-3}\right]$ in units of $R_{e}$, the end-to-end distance of the chain.

\section{implicit solvent}

The number of molecules of the surrounding solvent is much greater than the number of lipids and if it were included explicitly, it would demand most of the computational time during a simulation. Most of these molecules do not interact directly with the membrane. A common practice to reduce the calculation time is to integrate out the solvent degrees of freedom. The interactions between the monomers are hence described by an effective Hamiltonian. This approach sums over all the interactions between the particles to build a potential whose derivative furnishes an average force that, statistically, represents the hidden interaction with the "ghost" solvent molecules. 
The effective Hamiltonian of the system is described as a functional of the density. The functional of the density can be calculated assigning a value of the density on a three dimensional lattice Daoulas and Müller (2012)] while to set a particle-based simulation (and retain translational invariance) we express the densities in terms of local densities around each particle [Hömberg and Müller (2010)].

We can obtain the equation of state as previously defined by writing the Hamiltonian as an expansion up to the third order in density. The functional formulation of this potential is

$$
\frac{H_{n b}[\rho]}{k_{B} T}=\sum_{\alpha, \beta, \gamma \in\{A, B\}} \int \frac{\mathrm{d}^{3} r}{R_{e}^{3}}\left(\frac{v_{\alpha \beta}^{2}}{2} \rho_{\alpha} \rho_{\beta}+\frac{v_{\alpha \beta \gamma}^{3}}{3} \rho_{\alpha} \rho_{\beta} \rho_{\gamma}\right)
$$

where the Greek indices specify the type of the species. We can see how this Hamiltonian leads us to the same equation of state. In the next sections we are going to show that the Hamiltonian of (eq:1.3) can be written as pairwise potential (eq:1.43) and hence the total potential and force of the system are:

$$
H_{n b}[\rho]=\sum_{i, j<i} H_{n b}\left(r_{i j}\right) \quad \boldsymbol{F}^{t o t}=\sum_{i, j<i} \boldsymbol{F}_{i j}=-\sum_{i, j<i} \nabla_{\boldsymbol{r}_{i j}} H_{n b}\left(r_{i j}\right)
$$

The pressure is given by the partial derivative of the free energy with respect to the volume.

$$
P=-\frac{\partial}{\partial V} F=k_{B} T \frac{\partial}{\partial V} \ln Z \quad Z=\int \mathrm{d} \boldsymbol{r}^{N} e^{-\beta H_{n b}\left(r^{N}\right)}
$$

Since the potential does not depend on direction, we perform the following substitution

$$
\boldsymbol{r}=V^{1 / 3} \boldsymbol{r}^{\prime}
$$

The scaled positions $\boldsymbol{r}^{\prime}$ have the property that the scaled coordinates of all particles remain fixed even if we expand or contract the system. If we consider only one species $\alpha=\beta=\gamma=A$ substituting, we obtain

$$
v_{2}=v_{A A}, v_{3}=v_{A A A} .
$$

$$
P=k_{B} T \partial_{V} \ln \left(V^{N} \int \mathrm{d}{\boldsymbol{r}^{\prime N}}^{-\beta H_{n b}\left(V^{N / 3} r^{N}\right)}\right)=k_{B} T \rho-\left\langle\partial_{V} H_{n b}\left(V^{N / 3} r^{\prime N}\right)\right\rangle
$$

We can hence rewrite the last term of the previous equation as:

$$
r_{i j}=V^{1 / 3} r_{i j}^{\prime}, \partial V=\frac{3 V}{r_{i j}} \partial r_{i j}
$$

$$
\left\langle\partial_{V} H_{n b}\left(V^{N / 3} r^{\prime}\right)\right\rangle=\frac{1}{3 V}\left\langle\sum_{i, j<i} \boldsymbol{r}_{i j} \nabla_{\boldsymbol{r}_{i j}} H_{n b}\left(r_{i j}\right)\right\rangle=-\frac{1}{3 V}\left\langle\sum_{i, j<i} \boldsymbol{r}_{i j} \boldsymbol{F}_{i j}\right\rangle=:-\frac{W}{V}(1.8)
$$

where we have introduce the virial $W$. In the previous calculation we exploit the equivalence of ensembles since thermodynamics properties can be calculated as averages in any convenient ensemble Allen and Tildesley (1991)]. Substituting the Hamiltonian (1.3) the virial is:

$$
\begin{aligned}
-W & =\left\langle\partial_{V} H_{n b}\left(V^{N / 3} r^{N}\right)\right\rangle=V k_{B} T\left\langle\partial_{V} \int \frac{\mathrm{d}^{3} r}{R_{e}^{3}}\left(\frac{v_{2}}{2} \rho_{A}^{2}+\frac{v_{3}}{3} \rho_{A}^{3}\right)\right\rangle \\
& =V k_{B} T\left(\frac{v_{2}}{2} \rho_{A}^{2}+\frac{2 v_{3}}{3} \rho_{A}^{3}\right)
\end{aligned}
$$

yielding a third-order equation of state in mean field approximation.

A molecular dynamics simulation integrates the equations of motion of a system of particles. We must hence provide a description of forces acting between a certain number of particles. The density functional formulation of the interaction Hamiltonian in 1.3 in terms of the local density, $\rho$, provides a definition of the forces that act 
between the particles. In our model, an amphiphilic chain is described by a beadspring model with two different types of beads that we mark $A$, for a hydrophobic bead, and $B$ for a hydrophilic one. Neighboring beads along the backbone of the chain molecule are connected by harmonic springs. In our model the conservative interactions, $F^{c}$, are the sum of bonded, $b$, and non-bonded, $n b$, forces.

$$
\boldsymbol{F}^{c}=\boldsymbol{F}^{n b}+\boldsymbol{F}^{b}
$$

\section{discretisation}

The Hamiltonian of the system is invariant with respect to changing of the discretisation (number of beads per chain, $N_{b}$ ). This means that we have to rescale the virial coefficients with respect to the length scale, $R_{e}$, and to the discretisation, $N$, used.

The length scale, $R_{e}$, denotes the mean-square end-to-end distance of the lipid. For a freely-jointed model one obtains:

$$
\left\langle R_{e}^{2}\right\rangle=\sum_{i j}\left\langle\boldsymbol{r}_{i} \boldsymbol{r}_{j}\right\rangle=l^{2} \sum_{i j}\left\langle\cos \theta_{i j}\right\rangle=l^{2} \sum_{i j} \delta_{i j}=l^{2} N_{b}
$$

using $\left\langle\cos \theta_{i \neq j}\right\rangle=0 . \quad l$ is the statistical length. Its value depends on the chemical structure of the monomers and it is known and tabulated for a variety of different polymer materials [Mark (2007)]. The volume and the density of the system are rescaled in units of the end-to-end distance, $R_{e}$, and the number of beads per chain, $N_{b}$.

$$
V \mapsto \frac{V}{R_{e}{ }^{3}} \quad \rho \mapsto \rho \frac{R_{e}{ }^{3}}{N_{b}}
$$

This implies that the virial coefficients depend on the end-to-end distance and on the discretisation to preserve the invariance of the Hamiltonian.

$$
\begin{aligned}
\frac{H_{n b}[\rho]}{k_{B} T} & =\int \frac{\mathrm{d}^{3} r}{R_{e}{ }^{3}}\left(\frac{v_{2}^{\prime}}{2} \rho^{\prime 2}+\frac{v_{3}^{\prime}}{3} \rho^{\prime 3}\right)= \\
& =\int \mathrm{d}^{3} r\left(\frac{v_{2}^{\prime} R_{e}^{3}}{2 N_{b}} \frac{\rho^{\prime 2} N_{b}^{2}}{R_{e}^{6}}+\frac{v_{3}^{\prime} R_{e}^{6}}{3} \frac{\rho^{\prime 3} N_{b}^{3}}{R_{e}^{9}}\right)=\int \mathrm{d}^{3} r\left(\frac{v_{2}}{2} \rho^{2}+\frac{v_{3}}{3} \rho^{3}\right)
\end{aligned}
$$

i.e. the virial coefficients and the density scale with the following relations

$$
\rho \mapsto \frac{\rho R_{e}^{3}}{N_{b}} \quad v_{\alpha \beta} \mapsto \frac{v_{\alpha \beta} N_{b}^{2}}{R_{e}^{3}} \quad v_{\alpha \beta \gamma} \mapsto \frac{v_{\alpha \beta \gamma} N_{b}^{3}}{R_{e}^{6}}
$$

Also the bonded interactions depend on the discretisation. For computational simplicity, we do not utilise a freely jointed chain model with a fixed bond length but successive monomers are bonded by a harmonic potential. This is the minimal model that gives rise to Gaussian statistics of the chain conformations. The bonded interac$\mathbf{b}_{i}$ bond vector, $\mathbf{b}_{i}=\mathbf{r}_{i}-\mathbf{r}_{i+1}$, tions are given by a discredited Edwards-Hamiltonian:

$$
k_{s p}:=\frac{3\left(N_{b}-1\right)}{R_{e}^{2}} \text {. }
$$

$$
H_{b}^{s p}\left(\boldsymbol{b}_{i}\right)=\sum_{i=1}^{N_{b}-1} \frac{k_{s p}}{2}\left(\boldsymbol{r}_{i}-\boldsymbol{r}_{i+1}\right)^{2}
$$

Since the lipid chains are less flexible than long polymers and exhibit fewer conforma$\theta_{i}$ angle between two segments,

$$
\theta_{i}:=\operatorname{acos} \frac{-\boldsymbol{b}_{i} \boldsymbol{b}_{i+1}}{b_{i} b_{i+1}}
$$

$$
H_{b}^{b}\left(\theta_{i}\right)=k_{b} \sum_{i=2}^{N_{b}-1}\left(1-\cos \theta_{i}\right)
$$




\section{virial coefficients}

The Hamiltonian of non-bonded interaction considers interactions between beads of the same species, $A-A, B-B$, and cross interactions $A-B$. We must hence estimate seven virial coefficient: $v_{A A}, v_{A B}, v_{B B}, v_{A A A}, v_{B B B}, w_{A B B}$ and $w_{A A B}$. The virial coefficients characterise the different interactions between the species of beads and parametrise the equation of state. The hydrophilic head groups are in good solvent condition. A polymer in good solvent condition can be represented by a chain in which only the two-body interactions are relevant and hence, $v_{B B}>0$ and $v_{B B B}=0$, Fredrickson et al. (2002)]. For the hydrophobic tails higher orders are to be take into account. We consider a polymer melt composed by hydrophobic chains. The first coefficient represent the ideal gas and is equal to one, $v_{A}=1$. An equation of state up to the third order, which is capable of describing the coexistence between a dense hydrophobic melt and its vapor, requires that the second-order term is negative, $v_{A A}<0$, and the third is positive, $v_{A A A}>0$. The second- and third-order coefficients can be determined from the following reasoning. A first equation is provided by the fact that in an implicit solvent the external pressure at the coexistence approximately vanishes and the melt in equilibrium has the same pressure

$$
P \simeq 0 \simeq \rho_{\text {coex }}+\frac{v_{A A}}{2} \rho_{\text {coex }}^{2}+\frac{2 v_{A A A}}{3} \rho_{\text {coex }}^{3}
$$

A second equation is obtained from the isothermal compressibility defined by

$$
\frac{\beta}{k_{T}}=-V \beta\left(\frac{\partial P}{\partial V}\right)_{T}=\rho_{c} \partial_{\rho_{c}}\left(\rho_{c}+\frac{v_{A A}}{2} \rho_{c}^{2}+\frac{2 v_{A A A}}{3} \rho_{c}^{3}\right)=\rho_{c}+v_{A A} \rho_{c}^{2}+2 v_{A A A} \rho_{c}^{3}
$$

We define the dimensionless inverse compressibility, $k N_{b}$ :

$$
k N_{b}:=\beta \frac{\partial W}{\partial \rho_{c}}=\beta \frac{\partial W}{\partial \rho} N_{b} \quad \frac{1}{k_{T}}=\rho_{c} k_{B} T+\rho_{c} k_{B} T k N_{b}
$$

For a homopolymer melt, the thermal compressibility is given by:

$$
\frac{\beta}{k_{T}}=\rho \partial_{\rho} P=\rho \partial_{\rho}\left(\rho+\left\langle\partial_{\rho} H[\rho]\right\rangle\right)=\rho k_{B} T\left(1+\partial_{\rho} W\right)
$$

where we recall the definition of the virial in eq. 1.8. The thermal compressibility is also defined in terms of the Edwards correlation length, $\xi$ Wu et al. (1995)]

$$
\frac{\beta}{k_{T}}=\frac{\rho}{12\left(\xi / R_{e}\right)^{2}}
$$

The characteristic length of density fluctuations in the solution is the Edwards correlation length which is connected to the statistical segment length of an ideal chain, $b$, and the excluded volume, $v$, (positive in good-solvent conditions) [Meyer et al. (2008)].

$$
\xi=\frac{b}{(12 \rho v)^{1 / 2}}
$$

The mixed terms can be calculated using the theory of miscibility between two different species. Entropy favours the miscibility of the two components, but the system's repulsive forces tend to separate them. The Flory interaction parameter, $\chi$, characterises the difference of interaction energies in the mixture. The theory considers a blend of two different components, the component $A$ and the component $B$ which are chemically different. Component $A$ occupies the volume $V_{A}$ and $\phi_{A}$ is the volume fraction of the $A$ species, $\phi_{A}:=V_{A} / V_{t o t}$. The free energy of mixing, $\Delta F_{m}$, is the change in energy when two different chemical substances are mixed. The free energy of mixing for pure components, considered separated, is the sum of an entropic, $\Delta S$, and an 
enthalpic term, $\Delta E$. If the volume is totally occupied by the two species $\phi_{a}=\phi$ and $\phi_{b}=1-\phi$

$$
\frac{\Delta F_{m}}{k_{B} T}=\Delta E_{m}-T \Delta S=\frac{\phi}{N_{A}} \ln \phi_{A}+\frac{(1-\phi)}{N_{B}} \ln \phi_{B}+\phi(1-\phi) \chi
$$

The Flory-Huggins equation, on the right hand side, is the sum of combinatorial and interaction terms. Following Müller (1999); Flory (1941)] we can define the $\chi$ parameter by calculating the difference of the chemical potential per monomer between the two species. Neglecting fluctuations we can define $\chi$ as:

$$
\chi=\rho \int \mathrm{d}^{3} r\left(g_{A B}(\boldsymbol{r}) U_{A B}(\boldsymbol{r})-\frac{g_{A A}(\boldsymbol{r}) U_{A A}(\boldsymbol{r})+g_{B B} U_{B B}(\boldsymbol{r})}{2}\right)
$$

where $g_{\alpha \beta}$, with $\alpha, \beta \in\{A, B\}$, is the pair correlation function between the monomers of the species $\alpha$ with monomers of species $\beta$, and $U_{\alpha \beta}$ is the pair-wise potential energy between the two species. In a mean-field approximation, the pair correlation function is $g(\boldsymbol{r})=1$ and the integration of the potential yields the second-order virial coefficients.

$$
\begin{aligned}
\chi & \simeq \frac{\rho}{k_{B} T} \int \mathrm{d}^{3} r\left(U_{A B}(\boldsymbol{r})-\frac{1}{2}\left(U_{A A}(\boldsymbol{r})+U_{B B}(\boldsymbol{r})\right)\right)= \\
& =\rho\left(v_{A B}-\frac{1}{2}\left(v_{A A}+v_{B B}\right)\right)
\end{aligned}
$$

In Helfand's model, Helfand and Tagami (1971)], the previous theory is extended for a nearly incompressible system $\phi_{A}+\phi_{b} \lesssim 1$

$$
\frac{H_{I}}{k_{B} T}=\rho \int \mathrm{d}^{3} r\left(\chi \phi_{A}(r) \phi_{B}(r)+\frac{k_{m e l t}}{2}\left(\phi_{A}(r)+\phi_{B}(r)-1\right)^{2}\right)
$$

where the $k_{\text {melt }}$ parameter expresses the tendency of the system to pull the polymers into regions where the total density is $\rho_{A}+\rho_{B}=\rho_{0}$.

The density of the system, $\rho_{0}$, depends on the number of beads per chain, $N_{b}$. If we change the discretisation, the number density of beads in the system will change. Since $\rho_{0} k_{m e l t}$ and $\rho_{0} \chi$ are invariant, if we use units of the chain density $\rho_{c}=\rho_{0} / N_{b}$, $k N_{b}$ and $\chi N_{b}$ are invariant. Combining (1.17), 1.18 and 1.25 we obtain the virial coefficients

$$
\begin{array}{r}
v_{A A}=-2 \frac{k N_{b}+3}{\rho_{0}} \quad v_{A A A}=\frac{3}{2} \frac{k N_{b}+2}{\rho_{0}^{2}} \\
v_{A B}=\frac{\chi N_{b}}{\rho_{0}}+\frac{1}{2}\left(v_{A A}+v_{B B}\right)
\end{array}
$$

The evaluation of the other parameters is empirical. The remaining two third-order mixed terms $v_{A B B}, v_{A A B}$, should be positive and for simplicity we set them to the $v_{A A A}$ term: $v_{A A A}=v_{A A B}=v_{A B B}$. The hydrophilic beads are in good solvent conditions, i.e. there is no fluid/vapor phase separation. We hence set $v_{B B B}=0$ and $-0.5<$ $v_{B B}<0.1$. The $v_{B B}$ virial coefficient comprises as well the interactions with the missing water molecules and therefore we allow a small attraction between head groups (negative values per $v_{B B}$ ) to reproduce different hydration of the lipid head groups. For negative values of the coefficient $v_{B B}$ the head groups are slightly attractive between each other but do not show any cluster formation at the low hydrophilic densities of the usual simulated systems.

\section{3 dissipative particle dynamics}

To simulate the dynamics of polymers or lipids in the membrane we use the DPD DPD Dissipative Particle Dynamics

(Dissipative Particle Dynamics) simulation method. The original work from Koeleman 
and Hoogerbrugge (1993)] was successive upgraded by Pagonabarraga and Frenkel (2001)] into a MDPD (Multi body Dissipative Particle Dynamics) scheme, which we explain below.

DPD is a method to integrate the equations of motion, like molecular dynamics simulations but its range of validity is the mesoscopic scale and therefore requires Brownian noise which includes hidden interactions with the "ghost" microscopic particle. In contrast to a Langevin description, DPD uses noise and friction that locally conserve angular and linear momentum. Conservation of the hydrodynamics is important in annealing defects Gonnella et al. (1997)]. For the DPD thermostat, the equation of motion is the sum of conservative $(c)$, dissipative $(d)$ and random $(r)$ forces

$$
m \dot{\boldsymbol{v}}=\boldsymbol{F}^{c}+\boldsymbol{F}^{d}+\boldsymbol{F}^{r}
$$

Every component is limited to a distance interval, $\Delta L$, by a weighting function $w\left(r_{i j}\right)$ that depends on the relative distance between the particles. This function is 1 for $r=0$ and goes to zero at the cut-off distance $r=\Delta L$. Every force is pair-wise and thus locally conserves momentum.

$$
m \dot{\boldsymbol{v}}=w^{c}\left(r_{i j}\right) \boldsymbol{f}_{i j}^{c}+w^{d}\left(r_{i j}\right) \boldsymbol{f}_{i j}^{d}+w^{r}\left(r_{i j}\right) \boldsymbol{f}_{i j}^{r}
$$

The weighting function makes the forces soft and permits increase of the time-step of the simulated system [Pastorino et al. (2007)].

The conservative force depends on the particular system and it is obtained from the derivative of the potential. The dissipative and random forces should obey the fluctuation-dissipation theorem. If we determine a dissipative term $\gamma$ we have to define the strength, $\xi$. As shown in Orlandini [2008)], if we choose an uncorrelated Gaussian random noise with zero average

$$
\left\langle\theta_{i j}^{g}(t)\right\rangle=0 \quad\left\langle\theta_{i j}^{g}(t) \theta_{k l}^{g}\left(t^{\prime}\right)\right\rangle=\left(\delta_{i k} \delta_{j l}+\delta_{i l} \delta_{j k}\right) \delta\left(t-t^{\prime}\right)
$$

the strength terms should satisfy the relation

$$
\xi=\sqrt{2 \frac{\gamma k_{B} T}{m \Delta t}}
$$

In practise, following [Dünweg and Paul (1991)], we can use a uniform random number generator, $\theta_{i j}^{u}$, which is faster to compute, instead of a Gaussian. In this case we note that if a Gaussian distribution has a variance of $\sigma$, a uniform distribution equal to 1 between $[-\sigma / 2, \sigma / 2]$ has a variance $\sigma / \sqrt{12}$. Hence, our equation of motion takes the form

$$
m \dot{\boldsymbol{v}}=\boldsymbol{f}_{i j}^{c} w^{c}\left(r_{i j}\right)-\gamma w^{d}\left(r_{i j}\right)\left(\boldsymbol{v}_{i j} \hat{\boldsymbol{r}}_{i j}\right) \hat{\boldsymbol{r}}+\sqrt{\frac{24 \gamma k_{B} T}{m \Delta t}} \theta_{i j}^{u} w^{r}\left(r_{i j}\right)
$$

In Groot and Warren's work [Groot and Warren (1998)] the DPD simulation method is widely investigated and following their results, we set $\gamma=0.1$ and $\Delta t=0.01$ in the system's units: $k_{B} T=\Delta L=m=1$. On the suggestion of the same work we use the velocity Verlet integration scheme instead of the Euler's. Español and Warren (1995) Espanol and Warren (1995)] have shown that the dissipative weighting function can be chosen arbitrarily and is connected to the random weighting function by the relation $w^{d}(r)=\left(w^{r}(r)\right)^{2}$. In Pastorino et al. (2007)] different weighting functions in different polymer system are tested. The particular choice of a weighting function concerns the thermostat of the system and the computational efficiency. We define the number of particles thermostated, i.e. the particles included in the sphere within the cut-off multiplied by the weighting function, as

$$
N_{T P}=\rho_{0} \int_{0}^{r} w^{r}(r) g(r) 4 \pi r^{2} \mathrm{~d} r
$$

MDPD Multi-body Dissipative Particle Dynamics $w\left(r_{i j}\right) \boldsymbol{f}_{i j}$ strength of the force acting between the $i$ and $j$ particle, $\boldsymbol{r}_{i j}$ distance between the $i$ and $j$ particle. 
where the $g(r)$ in the pair correlation function that in our case we approximate as 1 . The use of the following weighting function

$$
w^{d}(r)=\left(w^{r}(r)\right)^{2}=\left(1-\frac{r}{\Delta L}\right)^{2} \quad \text { if } \quad r<\Delta L
$$

provides a fast computing efficiency. In agreement with the suggestions of Hömberg and Müller (2010)] and Trominov et al. (2002)], we observe in our simulations that if the cut-off distance include an average number of thermostated particle larger than 3-4 the system conserves temperature keeping a time step of $\Delta t=0.01$.

The integration of the equations of motion is performed via a velocity Verlet algorithm which is composed of two steps

$$
\boldsymbol{r}_{i}(\Delta t)=\boldsymbol{r}_{i}(0)+\Delta t \dot{\boldsymbol{r}}_{i}(0)+\frac{\Delta t^{2}}{2 m_{i}} \boldsymbol{F}_{i}(0) \quad \dot{\boldsymbol{r}}_{i}(\Delta t / 2)=\dot{\boldsymbol{r}}_{i}(0)+\frac{\Delta t}{2 m_{i}} \boldsymbol{F}_{i}(0)
$$

and secondly

$$
\dot{\boldsymbol{r}}_{i}(\Delta t)=\dot{\boldsymbol{r}}_{i}(\Delta t / 2)+\frac{\Delta t}{2 m_{i}} \boldsymbol{F}_{i}(\Delta t)
$$

and its derivation is briefly discussed in the appendix.

\section{multibody dissipative particle dynamics}

As was noted in Trominov et al. (2002)], the DPD model, as it was initially formulated, does not take into account the local density of the system. If the conservative forces depend only on the mutual distance between the particles, then the system produces an equation of state of up to second order [Groot and Warren (1998)]. This means that we can not simultaneously map the pressure and the compressibility of the system, because the inverse of compressibility is limited by the value of the pressure

$$
k_{T}^{-1}=\rho k_{B} T+v_{A A} \rho^{2}=2 P-\rho k_{B} T<2 P \quad P=\rho k_{B} T+\frac{v_{A A}}{2} \rho^{2}
$$

which is not the case for more compressible liquids. Pagonabarraga and Frenkel (2001)] proposed to redefine the conservative forces dependent upon the local density of the system. This suggests defining the free energy as a functional of the density and to proceed with the calculation of the conservative forces by deriving the free energy with respect to the spatial coordinates.

\section{conservative force}

The non-bonded interactions between the beads should come from the negative derivative of the potential.

$$
\boldsymbol{F}_{\alpha \beta}\left(r_{i}, r_{j}\right)=-\nabla_{\boldsymbol{r}} U\left(r_{\alpha}, r_{\beta}\right)
$$

where the Greek indices refer to the different types of beads, $A, B$. The forces depend on the type of beads involved and on their mutual distances. It is now important to show how we obtain a pairwise interaction, which is required by the DPD simulation scheme. The Hamiltonian we use for a one component system is, $v_{2}:=v_{A A}, v_{3}:=v_{A A A}$

$v_{2}:=v_{A A}$

$v_{3}:=v_{A A A}$

$$
\frac{H_{n b}[\rho]}{k_{B} T}=\int \mathrm{d}^{3} r\left(\frac{v_{2}}{2} \rho^{2}(\boldsymbol{r})+\frac{v_{3}}{3} \rho^{3}(\boldsymbol{r})\right)
$$

If we consider our particles to be points, we write the density as a sum of delta functions

$$
\rho(\boldsymbol{r})=\sum_{i} \delta\left(\boldsymbol{r}-\boldsymbol{r}_{i}\right)
$$


Since the product of delta functions is not defined, we rewrite the product of density functions as a delta times a weighting function $w(r)$

$$
\begin{aligned}
\rho^{2}(\boldsymbol{r}) & :=\sum_{i j} \delta\left(\boldsymbol{r}_{i}-\boldsymbol{r}\right) w\left(\left|\boldsymbol{r}-\boldsymbol{r}_{j}\right|\right) \\
\rho^{3}(r) & =: \quad \sum_{i j k} \delta\left(\boldsymbol{r}_{i}-\boldsymbol{r}\right) w\left(\mid \boldsymbol{r}-\boldsymbol{r}_{j}\right) w\left(\left|\boldsymbol{r}-\boldsymbol{r}_{k}\right|\right)
\end{aligned}
$$

This weighting function should be normalised by

$$
4 \pi \int_{0}^{\Delta L} \mathrm{~d} r r^{2} w(r)=1
$$

which helps us formulate the Hamiltonian as a sum over all the positions of the particles, $r_{i j}:=\left|\boldsymbol{r}_{i}-\boldsymbol{r}_{j}\right|$ $r_{i j}=\left|\boldsymbol{r}_{i}-\boldsymbol{r}_{j}\right|$

$$
\begin{aligned}
\sum_{i, j<i} \frac{H_{n b}\left(r_{i j}\right)}{k_{B} T} & =\int \mathrm{d}^{3} r \sum_{i j} \delta\left(\boldsymbol{r}-\boldsymbol{r}_{i}\right)\left(\frac{v_{2}}{2} w\left(\left|\boldsymbol{r}-\boldsymbol{r}_{j}\right|\right)+\frac{v_{3}}{3} w\left(\left|\boldsymbol{r}-\boldsymbol{r}_{j}\right|\right) \sum_{k} w\left(\left|\boldsymbol{r}-\boldsymbol{r}_{k}\right|\right)\right) \\
& =\sum_{i, j<i}\left(\frac{v_{2}}{2} w\left(r_{i j}\right)+\frac{v_{3}}{3} w\left(r_{i j}\right) \sum_{k} w\left(r_{i k}\right)\right)
\end{aligned}
$$

The weighting function, $w(r)$, represents the smoothing operation, which implies that we cannot resolve the system below a certain length scale $\Delta L$. We finally obtain the conservative force by deriving the potential with respect to the coordinate $\boldsymbol{r}_{i}$

$$
\begin{aligned}
\boldsymbol{F}_{i}^{n b} & :=-\partial_{\boldsymbol{r}_{i}} \sum_{j} H_{n b}\left(r_{i j}\right) \\
& =-k_{B} T \sum_{j}\left(\frac{v_{2}}{2} \partial_{\boldsymbol{r}_{i}} w\left(r_{i j}\right)+\frac{v_{3}}{3}\left(\partial_{\boldsymbol{r}_{i}} w\left(r_{i j}\right)\right) \sum_{k} w\left(r_{i k}\right)+\frac{v_{3}}{3} w\left(r_{i j}\right) \partial_{\boldsymbol{r}_{i}} \sum_{k} w\left(r_{i k}\right)\right) \\
& =-k_{B} T \sum_{j}\left(\left(\frac{v_{2}}{2}+\frac{2}{3} v_{3} \sum_{k} w\left(r_{i k}\right)\right) w^{\prime}\left(r_{i j}\right) \frac{\boldsymbol{r}_{i j}}{\left|\boldsymbol{r}_{i j}\right|}\right) \\
& =\sum_{j} \boldsymbol{F}_{i j}
\end{aligned}
$$

Where we have used

$$
\sum_{j}\left[\partial_{\boldsymbol{r}_{i}} \sum_{k} w\left(r_{i k}\right)\right] w\left(r_{i j}\right)=\sum_{j} \sum_{k}\left[\partial_{i} w\left(r_{i k}\right)\right] w\left(r_{i j}\right)=\sum_{j}\left[\partial_{i} w\left(r_{i j}\right)\right] \sum_{k} w\left(r_{i k}\right)
$$

In this way we obtain a pairwise expression for computing the conservative force, as required by the MDPD simulation method.

\section{4 homopolymer melt}

As we have seen in the previous calculation, the weighting function should be differentiable, without singularities and fast to compute. From 1.44 we can see that the definition of the weighting function changes the interactions. The definition of the weighting function is connected to the pair-correlation function and the effective potential. This relation applies only to low densities as a consequence of assumption required in the virial expansion.

The Boltzmann factor between two particles is $\exp \left(-\beta U\left(r_{i j}\right)\right)$, which reduces to 1 if the particles do not interact. The difference between the Boltzmann factor of the interacting and non interacting particles is defined as the Mayer function.

$$
f(r):=e^{-\beta U(r)}-1
$$


It is a positive function for an attractive potential $(U(r)$ negative), negative for a repulsive potential and zero for no interaction. The negative integral of the Mayer function gives the excluded volume.

$$
V_{e x}:=\int \mathrm{d}^{3} r\left(1-e^{-\beta U(r)}\right)
$$

\section{setting the weighting function}

The weighting function is a key feature of the DPD thermostat and softens the conservative, dissipative and random forces, improving the integrability of the equation of motion. These functions are null at the cut-off distance, $\Delta L$, positive (grow continuously towards zero distances) and their first derivative must be continuous.

Figure 1.2: The change of the $a$ parameter in the weighting function, from $a=0.9$ to a $a=0.5$, decreases density oscillations in the profile of the liquid-vapour interface between a melt and vacuum.

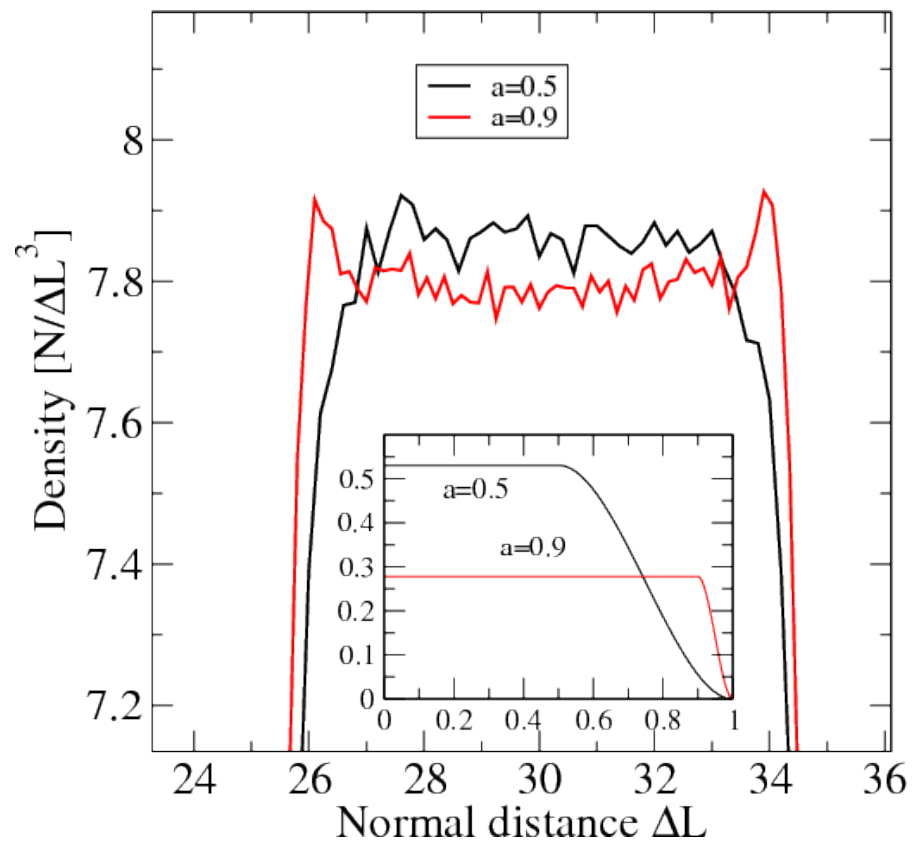

The weighting function changes the interactions and the choice of a particular weighting function can change the properties of the system, especially at the interfaces where the packing effect of the beads is most visible. The choice of the weighting function is constrained by computational efficiency. The fastest curves to compute that are constant within $[0, a]$ and are equal to zero at the cut-off distance, $\Delta L$, with no singularities in their derivative are the splines. The setting of the order of the spline and the limit of the $a$ value should represent the expected interaction.

We show as an example how the difference in the parameter of the weighting functions, Hömberg and Müller (2010)] (see eq[1.48, changes the density profile at the liquid vapour interface (see fig: 1.2 .

$$
w(r)=\left\{\begin{array}{cll}
\frac{2 r^{3}-3(a+1) x^{2}+6 a x-3 a^{2}+1}{(1-a)^{3}} & \text { if } & a<r<1 \\
0 & \text { if } & 1<r<a
\end{array}\right.
$$

The changing of the density profile with respect to the $a$ parameter can be explained in a heuristic way. If the $a$ parameter is large, the beads resemble hard spheres. The narrow liquid-vapour interface acts like a hard wall and gives rise to pronounced packing. If we make the hard core of the spheres more soft, decreasing $a$, the beads represent a soft volume rather than a hard sphere, and packing is no longer observed. In (see fig: 1.2), we observe that large values of a, such as $a=0.9$, show strong packing effect. 
To simulate a more realistic model of lipid membranes a distinction between the second- and third-order weighting function was introduced to reproduce fluid/gel phase transition [Hömberg and Müller (2010)]. The second-order interaction are attractive and we choose for the second-order weighting function $w_{2}(r)$ the function:

$$
w_{2}(r)=A\left\{\begin{array}{ccc}
(\Delta L)^{3} & \text { if } & 0 \leq r<a \\
\frac{2 r^{3}-3(a+1) x^{2}+6 a x-3 a^{2}+1}{(1-a)^{3}} & \text { if } & a<r<\Delta L \\
0 & \text { if } & r>\Delta L
\end{array}\right.
$$

$A=$

$\frac{-15}{2 \pi\left(2 a^{6}-3 a^{5} \Delta L+3 a \Delta L^{5}-2 \Delta L^{6}\right)}$, $a=0.9$.

The third-order interaction are repulsive and we define the third-order weighting function, $w_{3}(r)$, as:

$$
w_{3}(r)=\frac{15}{2 \pi}\left\{\begin{array}{cll}
(\Delta L-r)^{2} & \text { if } & r \leq \Delta L \\
0 & \text { if } & r \geq \Delta L
\end{array}\right.
$$

\section{5 bilayer membranes}

After having set the model and the simulation program and checked the properties of a homopolymer melt we start to form the amphiphilic polymers and lipids to create planar bilayer or vesicles. The mechanical properties and phase behaviour of those membranes strongly depend by the parameters of the model and on the chain architecture. In the following chapter we will extensively show how the material properties of the membrane are influenced by the model's parameters.

\section{notation}

In the following chapter we use the reduced units: $k_{B} T=1 / \beta=1$. For every value the error influences the last digit shown. If the calculation of a quantity, $v$, is the result of an interpolation and the interpolation is strongly subjected to the interval range, the range of the obtained values is written with this notation: $v_{1}-v_{2}$ where $v_{1}<v_{2}$. 


\section{Chapter 2}

\section{setting \\ the Monte Carlo simulations}

\section{1 lipid reservoir}

In most of the experimental conditions the size of the membrane is much larger than the portion studied by computer simulations. This means that a large amount of lipids is available to flow inside and outside the region of interest. The flow of lipids can be viewed as an exchange of the lipids of the system with a lipid reservoir. To extend the molecular dynamics simulations to the grand canonical ensemble we write a Monte Carlo code using the same model introduced in the previous chapter.

In the grand canonical ensemble the number of chains fluctuates. To vary the number of chains in the system we have to find a criterion to remove and insert the chains from and into the system. In the present chapter we introduce a modified Metropolis algorithm as sample method for allowing fluctuations in the number of chains. The chain is composed of beads connected in a line by a spring potential. To efficiently introduce a lipid into the system we have to construct the chain bead by bead and select the final chain among a set of different realisations. This procedure is called configurational bias and requires a modification of the Metropolis algorithm to restore the correct sampling.

To perform a grand canonical simulation we have to calculate the chemical potential of the lipids inside the bilayer starting from a sequence of snapshots obtained from the molecular dynamics simulations. The calculation of the chemical potential is done using the Shing-Gubbins method where we compare the probability distribution of the energy of the chains inside the bilayer with the probability distribution of the energy of inserting one chain in the same bilayer configuration.

Once we have set the simulation code, we run some test simulations to prove its validity and stability.

\section{2 grand canonical Monte Carlo for lipid chains}

In the grand canonical ensemble we consider a system of volume $V$, temperature $T=\beta=1$ and chemical potential of the chain $\mu_{c}$. In this ensemble the number of chains in the system $N_{c}$ is exchanged with a reservoir of ideal non interacting chains. An ideal chain has $N_{b}$ beads connected by an internal potential and does not interact with the neighboring chains. The partition function of a ideal chain is hence:

$$
Z_{c}:=Z_{c}\left(N_{b}, V, T\right)=\frac{1}{\Lambda^{3 N_{b}}} \int \mathrm{d} \mathbf{r}^{N_{b}} e^{-H_{s p}\left(r^{N_{b}}\right)}
$$


$\Lambda$ is the De Broglie thermal wave length, $\Lambda=\frac{h}{\sqrt{2 \pi m}}, h$ is the Planck constant, $m$ the mass of the particle.
The partition function of the system in the grand canonical ensemble is:

$$
Z_{g c}\left(\mu_{c}, V, T\right)=\sum_{N_{c}=0}^{\infty} \frac{e^{\mu_{c} N_{c}}}{\Lambda^{3 N} N_{c} !} \int \mathrm{d} \mathbf{r}^{N} e^{-H\left(\mathbf{r}^{N}\right)}
$$

In the grand canonical Monte Carlo method, every time step it is decided with equal probability whether to add or remove a chain from the system. The detailed balance condition states that the flow probability of inserting one chain in a system of $N_{c}$ chains (lhs) is equal to the flow probability of removing one chain from a $N_{c}+1$ chains system (rhs) de Pablo et al. (1992); Adams (1974, 1975, 1976, 1979)].

$P_{e q}(s)$ Probability at the equilibrium for the state $s$, $P_{\text {prop }}\left(s \rightarrow s^{\prime}\right)$ Probability of proposing a new state $s^{\prime}$, $P_{a c c}\left(s \rightarrow s^{\prime}\right)$ Probability of accepting the new state $s^{\prime}$,

$N:=N_{n} N_{c}$

$\mathbf{b}_{i}$ (bond vector)

$$
P_{e q}\left(N_{c}\right) P_{p r o p}^{+} P_{a c c}^{+}=P_{e q}\left(N_{c}+1\right) P_{p r o p}^{-} P_{a c c}^{-}
$$

We insert a new chain choosing randomly the position of the first bead and putting the consecutive monomers at a distance $\mathbf{b}_{i}$ (bond vector) from the previous one. The change in the coordinate system is:

$$
\mathbf{r}_{i}=\mathbf{r}_{1}+\sum_{j=1}^{i} \mathbf{b}_{j} \quad \mathbf{b}_{i}=\mathbf{r}_{i}-\mathbf{r}_{i-1} \quad \prod_{i=1}^{N_{b}} \mathrm{~d} \mathbf{r}_{i}=\mathrm{d} \mathbf{r}_{1} \prod_{i=2}^{N_{b}} \mathrm{~d} \mathbf{b}_{i}
$$

The determinant of the Jacobian is one as shown in the example $N=3$

$$
\operatorname{det}(J)=\left|\begin{array}{ccc}
1 & 0 & 0 \\
-1 & 1 & 0 \\
0 & -1 & 1
\end{array}\right|=1
$$

The partition function of a single chain is now

$$
\begin{aligned}
Z_{c} & =\frac{1}{\Lambda^{3 N_{b}}} \int_{0}^{V} \mathrm{~d} \mathbf{r}_{1} \prod_{l=2}^{N_{b}} \int \mathrm{d} \mathbf{b}_{l} e^{-\frac{3 \mathbf{b}_{l}^{2}}{2\left\langle b^{2}\right\rangle}}=\int_{0}^{V} \frac{\mathrm{d} \mathbf{r}_{1}}{\Lambda^{3 N_{b}}}\left(\int \mathrm{d} \mathbf{b} e^{-\frac{3 \mathbf{b}^{2}}{2\left\langle b^{2}\right\rangle}}\right)^{N_{b}-1} \\
& =: \frac{V}{\Lambda^{3 N_{b}}} C_{c}^{N_{b}-1}
\end{aligned}
$$

$C_{c}=\sqrt{2\left\langle b^{2}\right\rangle \pi}$ Where the normalisation constant is define as $C_{c}=\sqrt{2\left\langle b^{2}\right\rangle \pi}$.

To calculate the chemical potential of an ideal chain we consider a gas of ideal chains. $N$ total number of beads: Its partition function of the system in the canonical ensemble is:

$$
N=N_{b} N_{c} \text {. }
$$

$$
Z_{N_{c}}\left(N_{c}, V, T\right)=\frac{1}{\Lambda^{3 N} N_{c} !} \int \mathrm{d}^{N} \mathbf{r} e^{-H\left(\mathbf{r}^{N}\right)}
$$

Where we exclude the interactions between the chains:

$$
Z_{N_{c}}^{i d}\left(N_{c}, V, T\right)=\frac{1}{\Lambda^{3 N} N_{c} !}\left(\int \mathrm{d}^{N} \mathbf{r} e^{-H\left(\mathbf{r}^{N_{b}}\right)}\right)^{N_{c}}=\frac{1}{N_{c} !}\left(\frac{V C_{c}^{N_{b}-1}}{\Lambda^{3 N_{b}}}\right)^{N_{c}}
$$

Stirling's approximation: $\ln n ! \simeq n \ln n-n$

$$
F_{N_{c}}^{i d}\left(N_{c}, V, T\right)=-\ln Z_{N_{c}}=-\ln \frac{1}{N_{c} !}\left(\frac{V C_{c}^{N_{b}-1}}{\Lambda^{3 N_{b}}}\right)^{N_{c}} \simeq-N_{c} \ln \left(\frac{V C_{c}^{N_{b}-1}}{N_{c} \Lambda^{3 N_{b}}}\right)+N_{c}
$$

$\rho$ is the chain density. The chemical potential of an ideal chain is:

$$
\mu_{i d}:=-\frac{\partial F_{N_{c}}^{i d}}{\partial N_{c}}=-\frac{\partial}{\partial N_{c}} \ln Z_{N_{c}} \simeq \ln \left(\Lambda^{3} \rho\right)-\ln \frac{C_{c}^{N_{b}-1}}{\Lambda^{3\left(N_{b}-1\right)}}
$$


At each step we equally propose to insert or remove a molecule from the system. The probability of inserting the first bead is uniform all over the volume $V$ while the bonds are distributed normally choosing as variance the inverse of the harmonic spring constant.

$$
\prod_{l=2}^{N_{b}} e^{-\frac{3 \mathbf{b}_{l}^{2}}{2\left\langle b^{2}\right\rangle}}=e^{-\sum_{l=0}^{N-1} \frac{3 \mathbf{b}_{l}^{2}}{2\left\langle b^{2}\right\rangle}}=e^{-H_{s p}(c)} \quad H_{s p}(c)=\sum_{l} \frac{k_{s p}}{2} \mathbf{b}_{l}^{2}=\sum_{l} \frac{3 \mathbf{b}_{l}{ }^{2}}{2\left\langle b^{2}\right\rangle}
$$

where the variance of the Gaussian distribution $\left\langle b^{2}\right\rangle$ is chosen to be the inverse of the $k_{s p}=: \frac{3}{\left\langle b^{2}\right\rangle}$ elastic potential prefactor $k_{s p}$ and $H_{s p}(c)$ is the spring energy of chain $c$.

The probability to propose an insertion is then

$$
P_{\text {prop }}^{+}=\frac{1}{2} \frac{1}{V} \mathrm{~d} \boldsymbol{r} \frac{\prod_{l=2}^{N_{b}} e^{-\frac{k_{s p}}{2} \mathbf{b}_{l}^{2}} \mathrm{~d} v e t b_{l}}{C_{c}^{N_{b}-1}}
$$

The probability of a state with $N_{c}$ labeled chains is

$$
P_{e q}\left(N_{c}, \mathbf{r}^{N}\right)=\frac{1}{Z_{g c}\left(\mu_{c}, V, T\right)} \frac{e^{\mu_{c} N_{c}}}{\Lambda^{3 N}} e^{-H\left(\mathbf{r}^{N}\right)}
$$

The probability to remove one of the $N_{c}+1$ chains with the reverse move is

$$
P_{\text {prop }}^{-}=\frac{1}{2\left(N_{c}+1\right)} \mathrm{d} \boldsymbol{r} \prod_{l=2}^{N_{b}} \mathrm{~d} \boldsymbol{b}_{l}
$$

The lhs of the detailed balance 2.3 is now

$$
\frac{1}{2 V C_{c}^{N_{b}-1}} \frac{P_{a c c}^{+}}{Z_{g c}\left(\mu_{c}, V, T\right)} \frac{e^{\mu_{c} N_{c}}}{\Lambda^{3 N}} e^{-H\left(\mathbf{r}^{N}\right)-H_{s p}(c)}
$$

the rhs is

$$
\frac{1}{2\left(N_{c}+1\right)} \frac{P_{a c c}^{-}}{Z_{g c}\left(\mu_{c}, V, T\right)} \frac{e^{\mu_{c}\left(N_{c}+1\right)} e^{-H\left(\mathbf{r}^{N_{b}\left(N_{c}+1\right)}\right)}}{\Lambda^{3 N_{b}\left(N_{c}+1\right)}}
$$

From the last two equations the ratio between the acceptance criterion of insertion and removal of a chain is

$$
\frac{P_{a c c}^{+}}{P_{a c c}^{-}}=\frac{V C_{c}^{N_{b}-1}}{\Lambda^{3 N_{b}}\left(N_{c}+1\right)} e^{\mu_{c}} e^{-H\left(r^{N_{b}\left(N_{c}+1\right)}\right)+H\left(r^{N}\right)+H_{s p}(c)}
$$

We have set the ideal chemical potential of the chain, $\mu_{c}^{i d}$ as

$$
\mu_{c}^{i d}=\mu_{\text {dens }}^{i d}+\mu_{s p}^{i d}=\ln \rho \Lambda^{3}-\ln \frac{C_{c}^{N_{b}-1}}{\Lambda^{3\left(N_{b}-1\right)}}
$$

so that the excess part is defined as the difference $\mu_{c}^{e x}=\mu_{c}-\mu_{c}^{i d}$.

Defining the energy differences per chain $E^{+}(c), E^{-}(c)$ we can write the acceptance probability of insertion and removal like:

$$
\begin{aligned}
& P_{a c c}^{+}\left(N_{c} \rightarrow N_{c}+1\right)=\min \left(1, \frac{V \Lambda^{3}}{N_{c}+1} e^{\mu_{d e n s}^{i d}+\mu_{c}^{e x}} e^{-E^{+}(c)}\right) \\
& P_{a c c}^{-}\left(N_{c} \rightarrow N_{c}-1\right)=\min \left(1, \frac{N_{c}}{V \Lambda^{3}} e^{-\mu_{d e n s}^{i d}-\mu_{c}^{e x}} e^{+E^{+}(c)}\right)
\end{aligned}
$$

$\mu_{c}^{e x}=\mu_{c}-\mu_{c}^{i d}$

$E^{+}(c)=$ $H_{\overline{s p}}\left(r^{N_{b}\left(N_{c}+1\right)}\right)-H_{\overline{s p}}\left(r^{N_{b} N_{c}}\right)$, $E^{-}(c)=$ $H_{\overline{s p}}\left(r^{N_{b}}\left(N_{c}-1\right)\right)-H_{\overline{s p}}\left(r^{N_{b} N_{c}}\right)$ 


\section{3 configurational bias: insertion}

To improve the acceptance ratio for the insertion of chains we choose the position of every bead introducing the following bias: Mooij and Frenkel (1994); Smit (1995); Norizoe et al. (2010)]

- the first bead is put uniformly into the system box

- every consecutive bead is chosen among a set of different configurations with respect to its Boltzmann probability

- the chain is accepted with a probability given by the biased Metropolis algorithm

The bead $l$ of the chain $c$ is put at a distance $\mathbf{b}_{l}^{c}$ (bond vector) from the bead $l-1$. The bond vector $b_{l}^{c}$ is chosen among a set of $N_{b v}$ different bond vectors $\left(b_{l, i}^{c}\right)$ normally distributed with the probability

$$
P^{c}(\boldsymbol{b}) \mathrm{d} \boldsymbol{b}=\frac{e^{-\frac{k_{s p}}{2} \mathbf{b}^{2}}}{C_{c}} \mathrm{~d} \boldsymbol{b}
$$

Every bond vector has a Boltzmann factor $\exp \left(-E^{+}\left(\mathbf{b}_{l, i}\right)\right) / W_{c}^{l}$ where $E^{+}\left(\mathbf{b}_{l, i}\right)$ is the non-spring $(\overline{s p})$ added energy to the system due to the presence of the new particle and $W_{c}^{l}$ the normalization factor.

$$
E^{+}\left(\mathbf{b}_{l, i}\right)=H_{\overline{s p}}\left(r^{N} r^{l-1} r_{l, i}\right)-H_{\overline{s p}}\left(r^{N} r^{l-1}\right) \quad W_{c}^{l}:=\sum_{i=1}^{N_{b v}} e^{-E^{+}\left(\mathbf{b}_{i, l}\right)}
$$

In the configurational bias method the probability to propose an insertion is:

$$
\begin{aligned}
P_{\text {prop }}^{+} & =\frac{1}{2 V} \mathrm{~d} \mathbf{r}_{1}^{c} \prod_{l=2}^{N_{b}}\left(\frac{e^{-E^{+}\left(\mathbf{b}_{l}\right)}}{W_{c}^{l} / N_{b v}} \prod_{i=1}^{N_{b v}} P^{c}\left(\boldsymbol{b}_{l, i}\right) \mathrm{d} \boldsymbol{b}_{l, i}\right) \\
& =\frac{1}{2 V} \mathrm{~d} \mathbf{r}_{1}^{c} \prod_{l=2}^{N_{b}}\left(\frac{e^{-E^{+}\left(\mathbf{b}_{l}\right)}}{W_{c}^{l} / N_{b v}} \frac{\prod_{i=1}^{N_{b v}} e^{-\frac{k_{s p}}{2}\left(\mathbf{b}_{i, l}\right)^{2}} \mathrm{~d} \boldsymbol{b}_{i, l}}{C_{c}^{N_{b v}}}\right)
\end{aligned}
$$

The flow probability of the insertion in the configurational bias is:

$$
\frac{e^{\mu_{c} N_{c}-H\left(\mathbf{r}^{N_{b} N_{c}}\right)}}{Z_{g c}\left(\mu_{c}, V, T\right) \Lambda^{3 N_{b} N_{c}}} \frac{1}{2 V} \mathrm{~d} \mathbf{r}_{1}^{c} \prod_{l=2}^{N_{b}}\left(\frac{e^{-E^{+}\left(\mathbf{b}_{l}\right)}}{W_{c}^{l} / N_{b v}} \frac{\prod_{i=1}^{N_{b v}} e^{-\frac{k_{s p}}{2}\left(\mathbf{b}_{i, l}\right)^{2}} \mathrm{~d} \boldsymbol{b}_{i, l}}{C_{c}^{N_{b v}}}\right) P_{a c c}^{+}
$$

\section{4 configurational bias: deletion}

Similar to the insertion step described above, in the deletion step we choose randomly a chain from the system and for every bead of the chain we create a set of bond vectors $\left(\boldsymbol{b}_{l, i}\right)$ and calculate their energy $E^{+}\left(\mathbf{b}_{i, l}\right)$, and Boltzmann weight $W_{c}^{l}$ (see eq. 2.13). This is called super-detailed balance and equals the counter part in the flow probability of acceptance. The probability to propose a deletion is

$$
P_{\text {prop }}^{-}=\frac{1}{2\left(N_{c}+1\right)} \prod_{l=2}^{N_{b}}\left(\frac{\prod_{i=2}^{N_{b v}} e^{-\frac{k_{s p}}{2}\left(\mathbf{b}_{i, l}\right)^{2}} \mathrm{~d} \boldsymbol{b}_{i, l}}{C_{c}^{N_{b v}}}\right)
$$

since the probability to choose a monomer in the chain is 1 . We have chosen to assign $i=1$ to the set corresponding to the old chain conformation so that the product $\prod_{i=2}^{N_{b v}}$ 
runs only over the trial sets.

The flow probability of the deletion in the configurational bias is

$$
\frac{e^{\mu_{c}\left(N_{c}+1\right)-H\left(\mathbf{r}^{N_{b}\left(N_{c}+1\right)}\right)}}{Z_{g c}\left(\mu_{c}, V, T\right) \Lambda^{3 N_{b}\left(N_{c}+1\right)}} \frac{1}{2\left(N_{c}+1\right)} \prod_{l=2}^{N_{b}}\left(\frac{\prod_{i=2}^{N_{b v}} e^{-\frac{k_{s p}}{2}\left(\mathbf{b}_{i, l}\right)^{2}} \boldsymbol{b}_{i, l}}{C_{c}^{N_{b v}}}\right) P_{a c c}^{-}
$$

where the total energy of the system is

$$
H\left(r^{N_{b}\left(N_{c}+1\right)}\right)=H\left(r^{N_{b} N_{c}}\right)+E^{+}\left(\mathbf{r}_{1}\right)+\sum_{l=2}^{N_{b}} E^{+}\left(\mathbf{b}_{l}^{c}\right)+\sum_{l=2}^{N_{b}} \frac{k_{s p}}{2} \mathbf{b}_{l}^{2}
$$

\section{5 configurational bias: acceptance criterion}

According to the super-detailed balance, the set of trial orientations of the flow probability of insertion (eq2.15 equals the one of the deletion (eq2.17) and reduce the ratio between the acceptance criteria of insertion and deletion in:

$$
\frac{P_{a c c}^{+}}{P_{a c c}^{-}}=\frac{V e^{\mu_{d e n s}^{i d}}}{\left(N_{c}+1\right) \Lambda^{3}} W_{c} e^{\mu_{c}^{e x}}
$$

Where we have introduced the Rosenbluth weight to restore the correct sampling

$$
W_{c}:=e^{-E^{+}\left(\mathbf{r}_{1}^{c}\right)} \prod_{l=2}^{N_{b}} \frac{W_{c}^{l}}{N_{b v}}
$$

\section{6 added energy}

The Hamiltonian of the system is the sum of the non-spring $(\overline{s p})$ (non-bonded $(n b)$ and bending (ben)) and spring $(s p)$ potentials.

$$
H\left(r^{N}\right)=H_{\overline{s p}}\left(r^{N}\right)+H_{s p}\left(r^{N}\right) \quad H_{\overline{s p}}\left(r^{N}\right)=H_{n b}\left(r^{N}\right)+H_{b e n}\left(r^{N}\right)
$$

The non-bonded potential is express in terms of a density functional,

$$
H_{n b}\left(r^{N}\right)=\sum_{\alpha \beta \gamma} \int \mathrm{d} \mathbf{r} \frac{v_{\alpha \beta}}{2} \rho_{\alpha}(r) \rho_{\beta}(r)+\frac{v_{\alpha \beta \gamma}}{3} \rho_{\alpha}(r) \rho_{\beta}(r) \rho_{\gamma}(r)
$$

where $\alpha \beta \gamma$ iterate on the monomer type.

In a particle-based calculation we compute the local densities $\rho_{\alpha}\left(r_{i}\right)$ in terms of $\delta$ functions and weighting functions $w(r)$

$$
\begin{aligned}
\rho_{\alpha}\left(r_{i}\right)=\sum_{j}^{N} \delta\left(\mathbf{r}_{i}-\mathbf{r}_{j}\right) \delta_{t_{j}, \alpha} & \rho_{\alpha}\left(r_{i}\right) \rho_{\beta}\left(r_{i}\right)=\sum_{j k}^{N} \delta\left(\mathbf{r}_{i}-\mathbf{r}_{k}\right) w\left(\mathbf{r}_{i}-\mathbf{r}_{j}\right) \delta_{t_{j}, \alpha} \delta_{t_{k}, \beta} \\
\rho_{\alpha}\left(r_{i}\right) \rho_{\beta}\left(r_{i}\right) \rho_{\gamma}\left(r_{i}\right)= & \sum_{j k l}^{N} \delta\left(\mathbf{r}_{i}-\mathbf{r}_{j}\right) w\left(\mathbf{r}_{i}-\mathbf{r}_{k}\right) w\left(\mathbf{r}_{i}-\mathbf{r}_{l}\right) \delta_{t_{j}, \alpha} \delta_{t_{k}, \gamma}(2.22)
\end{aligned}
$$

We reformulate hence the non-bonded interactions as

$$
H_{n b}\left(r^{N}\right)=\sum_{\alpha \beta \gamma}\left(\frac{v_{\alpha \beta}}{2} \sum_{i j}^{N} w\left(r_{i j}\right) \delta_{t_{i}, \alpha} \delta_{t_{j}, \beta}+\frac{v_{\alpha \beta \gamma}}{3} \sum_{i j}^{N} w\left(r_{i j}\right) \sum_{i k}^{N} w\left(r_{i k}\right) \delta_{t_{i}, \alpha} \delta_{t_{j}, \beta} \delta_{t_{k}, \gamma}\right)
$$

Defining the local density for the particle $i, \rho_{i}^{\beta}:=\sum_{j}^{N} w\left(r_{i j}\right) \delta_{t_{j}, \beta}$ the non-bonded energy for the particle $i$ of type $t_{i}=\alpha$ is

$$
H_{n b}^{i \alpha}=\sum_{\beta} \frac{v_{\alpha \beta}}{2} \rho_{i}^{\beta}+\sum_{\beta \gamma} \frac{v_{\alpha \beta \gamma}}{3} \rho_{i}^{\beta} \rho_{i}^{\gamma}
$$


A ghost particle is represented by its position, type, and energy but it is not yet present in the system and its non bonded energy is

$$
\begin{aligned}
E_{g}^{\alpha} & =\frac{1}{2} \sum_{\beta} v_{\alpha \beta} \rho_{g}^{\beta}+\frac{1}{2} \sum_{\beta} \sum_{p} v_{\alpha \beta} w_{\alpha \beta}(p, g)+\frac{1}{3} \sum_{\beta} \sum_{\gamma} v_{\alpha \beta \gamma} \rho_{g}^{\beta} \rho_{g}^{\gamma} \\
& +\frac{1}{3} \sum_{\beta} \sum_{\gamma} \sum_{p}^{N} v_{\alpha \beta \gamma}\left(\rho_{p}^{\beta}+w_{\alpha \beta \beta}(g, p)\right)\left(\rho_{p}^{\gamma}+w_{\alpha \gamma \gamma}(g, p)\right)-\frac{1}{3} \sum_{\beta} \sum_{\gamma} \sum_{p}^{N} v_{\alpha \beta \gamma} \rho_{p}^{\beta} \rho_{p}^{\gamma} \\
& =\sum_{\beta} v_{\alpha \beta} \rho_{g}^{\beta}+\frac{1}{3} \sum_{\beta} \sum_{\gamma} v_{\alpha \beta \gamma} \rho_{g}^{\beta} \rho_{g}^{\gamma} \\
& +\frac{1}{3} \sum_{\beta} \sum_{\gamma} \sum_{p}^{N} v_{\alpha \beta \gamma}\left(\rho_{p}^{\beta} w_{\alpha \gamma \gamma}(g, p)+w_{\alpha \beta \beta}(g, p) \rho_{p}^{\gamma}+w_{\alpha \beta \beta}(g, p) w_{\alpha \gamma \gamma}(g, p)\right)
\end{aligned}
$$

\section{7 chemical potential of the molecules}

To calculate the chemical potential of a lipid we follow the method explained in the comprehensive works of Mooij and Frenkel (1994); Frenkel et al. (1991)]. It uses the Shing-Gubbins scheme [Shing and Gubbins (1982, 1981)] based on the test particle method of Widom Widom (1963)]. The chemical potential of a lipid is the free energy difference between two systems which differs from one lipid.

$$
\mu:=F\left(N_{c}+1, V, T\right)-F\left(N_{c}, V, T\right)=k T \ln \left(Z_{N_{c}} / Z_{N_{c}+1}\right)
$$

where $N_{c}$ is the number of molecules, $V$ the volume of the system, and $\mathrm{Z}$ the partition function of the system

$$
Z_{N_{c}}=\frac{1}{N_{c} ! \Lambda^{3 N}} \int_{V} \mathrm{~d} r^{N} e^{-\beta H\left(r^{N}\right)}
$$

Where $H\left(r^{N}\right)$ is the internal energy of a system of $N_{c}$ molecules.

The probability distribution of a certain energy difference between the system with $N_{c}$ and the system with $N_{c}+1$ molecules is

$$
\begin{aligned}
P^{-}(u) & =\left\langle\delta\left(u-H_{\overline{s p}}\left(r^{N_{b}\left(N_{c}+1\right)}\right)+H_{\overline{s p}}\left(r^{N_{b} N_{c}}\right)\right)\right\rangle_{N_{c}+1} \\
P^{+}(u) & =\frac{1}{V^{N_{c}}} \int_{V} \mathrm{~d} \mathbf{r}_{N_{c}+1}\left\langle\delta\left(u-H_{\overline{s p}}\left(r^{N_{b}\left(N_{c}+1\right)}\right)+H_{\overline{s p}}\left(r^{N_{b} N_{c}}\right)\right)\right\rangle_{N_{c}}
\end{aligned}
$$

Where $P^{-}(u)$ is the probability distribution of finding the energy $u$ among the ensemble average of all the non-spring energies, $H_{\overline{s p}}$, of a system of $N_{c}+1$ chains. $P^{+}(u)$ is the probability distribution of the energy between an added molecule in the position $\mathbf{r}_{N_{c}+1}$ and the remaining $N_{c}$.

To obtain $P^{+}(u)$ we calculate the energy of a molecule randomly put in the system. We start putting the first bead of the chain uniformly distributed in the simulation box $V$, the following beads are chosen following the distribution (see eq2.13). The excess part of the chemical potential is:

$$
\mu_{e x}=u+k_{B} T \ln \left(\frac{P^{-}(u)}{P^{+}(u)}\right)
$$

\section{8 simulation details}

Among all the possible system we could simulate we specifically want to set our simulations to represent the polymers created by Prof. Maskos of the University of Mainz 
Maskos (2006)]. These polymers are made of poly(butadiene) 130 -b-poly(ethylene oxide $_{66}$ diblock copolymers and spontaneously self-assembly in polymersome, whose phase and mechanical properties are widely investigated in their works W.Müller et al. (2008); Maskos (2006); Jungmann et al. (2003)].

\section{setting the interactions}

We estimate the input parameters of the interactions in the following way. We consider a chain of 66 monomers of ethylene oxide, $E O$, and 133 monomers of butadiene, $B$. The experimental value of the end-to-end distance of this chains is $R_{e}=8.14[\mathrm{~nm}]$. The thickness of the hydrophobic layer of the formed vesicle is $\bar{d}=16[\mathrm{~nm}] \simeq 2\left[R_{e}\right]$. The weight density of a melt of polybutadiene is around $0.94\left[\mathrm{~g} / \mathrm{cm}^{3}\right]$ from which we calculate the monomer density is $58 \cdot 10^{-3}\left[N_{m} / \mathrm{nm}^{3}\right]=31.3\left[N_{m} / \Delta L^{3}\right]$. The incompatibility between the two monomer species is give by the formula

$$
\chi N=\frac{\left(\delta_{B}-\delta_{E O}\right)^{2}}{k_{B} T} \frac{V}{N_{c}} \simeq 60.8
$$

where $\delta_{B}$ and $\delta_{E O}$ are the Hildebrandt solubility for polybutadiene and polyethylene oxide [Mark (2007)].

The asymmetry between the hydrophobic and hydrophilic beads is chosen with the ratio 5:1 (27 $A$ and $5 B$ beads) which is necessary for the self-assembly of the chain in a bilayer in the lamellar phase. The discretisation chosen to represent the coarse-grained chain is 32 which is a good compromise to represent the flexibility of the polymer chains and computational performances.

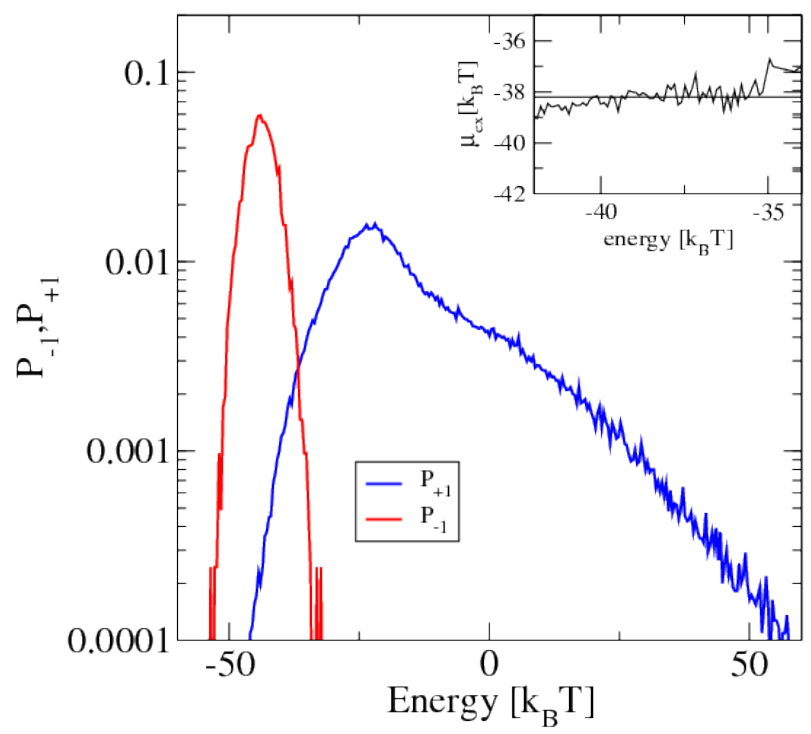

Figure 2.1: lhs) Probability distributions of insertion $\left(P^{+}(u)\right)$ and deletion $\left(P^{-}(u)\right)$ of molecules in a planar bilayer. In the inset the excess chemical potential $\left(\mu_{e x}\right)$ in the overlapping region is shown. The straight line in the insets shows the value of the calculated excess chemical potential: $\mu_{e x}=-38.2\left[k_{B} T\right]$.

We have created a stable bilayer in a simulation box of $32 \times 32 \times 16\left[\Delta L^{3}\right]$ volume using molecular dynamics simulations (described in the previous chapter) to create a sequence of equilibrated system configurations. From this sequence of snapshots we have calculate the chemical potential of the polymers using the Widom insertion method (see fig 2.1. 


\title{
2.9 test runs for Monte Carlo simulations
}

\author{
single chain comparison
}

We compare the statistical properties of the system with different simulation techniques.

FIGURE 2.2: Probability distribution of the: bonded energy (lhs), non bonded energy (rhs), and end-to-end distance (middle) using different simulation schemes (see text).
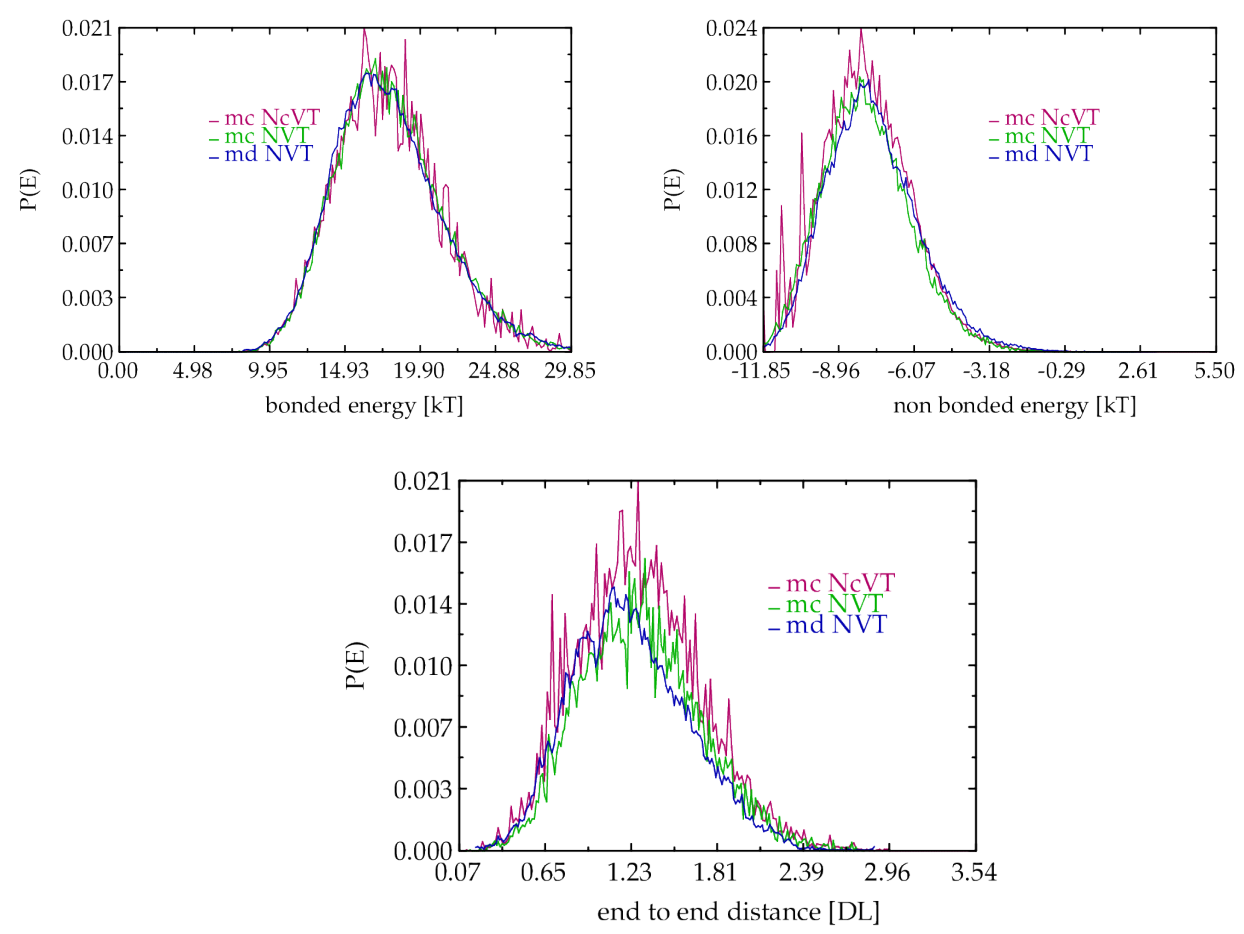

The system is composed by a single chain in the solvent. The simulation is run with: 1) molecular dynamics simulations, (md $N V T$ ), 2) Monte Carlo simulations displacing a single bead, (mc NVT), and 3) Monte Carlo simulations removing and constructing a new chain, (mc $N_{c} V T$ ). We study the probability distribution of the bonded and non bonded energy and the end-to-end distance (see fig 2.2 .

This simulation runs confirm the correctness of the calculation of the system energy and the structure of the chain.

\section{gas phase}

We simulate a system of non interacting lipids by considering only the internal energy of the chain.

This test run allows us to check the consistency between the simulations with and without configurational bias. In the gas phase the acceptance ratio is large and we can really in few simulation steps observe the equilibration of the system. We have measured that the excess chemical potential of a lipid in the gas phase is around 4.5 and $5\left[k_{B} T\right]$. We have first set the excess chemical potential, $\mu^{e x}$, to a value of $4.5\left[k_{B} T\right]$ and the ideal, $\mu_{\text {dens }}^{i d}=\ln \left(N_{c} / V \Lambda^{3}\right)$, where $N_{c}=150$. We observe that both grand canonical simulations, with and without configurational bias, stabilize to the same energy and number of chains (see fig 2.3). We can see from the graphs that the equilibration is faster in the case of the configurational bias. 

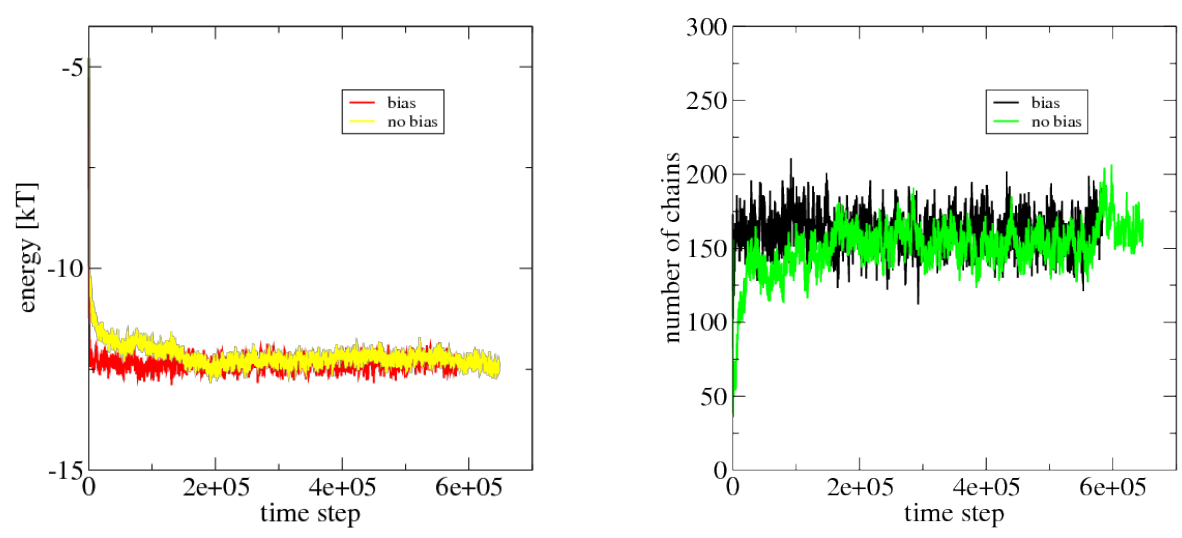

FiguRE 2.3: Time evolution of the energy (lhs) and number of chains (rhs) for the grand canonical simulations of lipids in the gas phase with or without configurational bias.

\subsection{1 lipids in a bilayer}

Once we have checked the consistency of the grand canonical simulations with and without bias we start a Monte Carlo simulation after an equilibrated molecular dynamics simulation. 
lipids in a bilayer 


\section{Chapter 3}

\section{inclusion stability}

Artificial hydrophobic compounds, like quantum dots or nanoparticles, can be stabilized in the hydrophobic layer of bilayer membranes. To design a compound that can remain stable inside the bilayer we study with the solvent-free coarse-grained model introduced before the stability conditions for such a compound. The hydrophobic compound is described by its size and hydrophobic coating, and we study the dependence of these parameters on its stability inside the bilayer.

The weak interactive nanoparticles exit from the membrane in a short time, the stable ones remains inside the membrane for a time at least three times longer than the average exit time of the weak interactive nanoparticles.

The energy barrier for the nanoparticle to exit from the membrane taking out a polymeric coating is too large to observe such an event during the simulation time. We compare hence the chemical potential of a nanoparticle inside the bilayer with a polymeric coated nanoparticle in the solvent and discuss the energetic stability of both conditions.

\section{1 energetic stability of a hydrophobic inclusion in bilayer membranes}

Small hydrophobic nanoparticles (like fluorescent dyes or quantum dots) can be embedded in the hydrophobic layer of small lipid or polymeric vesicles to trace their position. These vesicles can be equipped with ligand-proteins to dock to specific target tissues and trace in vivo the position of particular tissues. The traceability of the vesicle is hence tightly connected with the stability of the nanoparticle. We specifically map our system into the experimental setup of Prof. Maskos of the University of Mainz Maskos (2006)] who created self-assembled polymersome made of poly(butadiene) ${ }_{130^{-}}$ b-poly(ethylene oxide) 66 diblock copolymers.
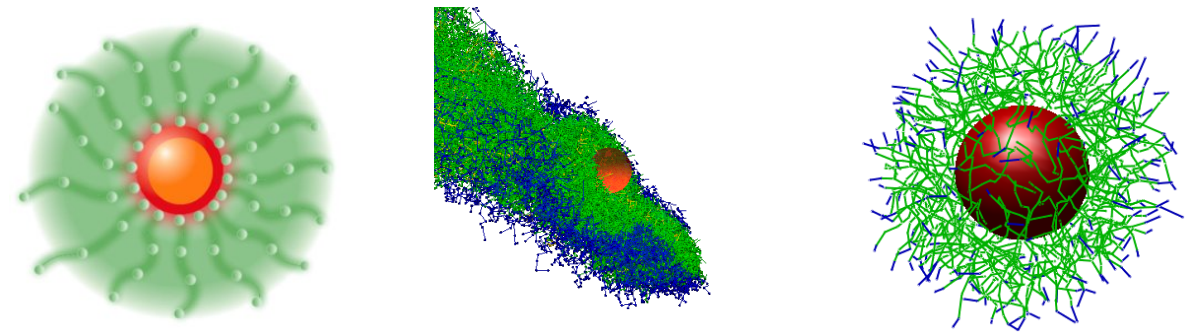

We use the solvent-free coarse-grained model introduced in the previous chapters to simulate with high computational efficiency large patches of polymeric membranes and explore the characteristic of a stable spherical hydrophobic inclusion (described by an integrated Lennard-Jones potential). The first part of the work consists in mapping the

Figure 3.1: Sketch of a nanoparticle, the interior represents the metallic core, the red layer the $\mathrm{ZnS}$ coating and the green chains the hydrophobic polymers (like oleic acid) grafted on the surface (lhs) to make the nanoparticle hydrophobic. Sketch of a nanoparticle embedded in the bilayer (middle). Sketch of a nanoparticle with a polymeric coating (rhs). 
specific system to the input parameters of the model using the data of the literature.

A nanoparticle is usual made of an hard core sphere (metallic core coated by CdSe or $\mathrm{ZnS}$ ) with hydrophobic polymers (oleic acid) grafted onto its surface.

The unstable nanoparticles are described by a weak interaction and exit the membrane in a short time.

The stable nanoparticles were completely surrounded by the polymers for a time at least three times longer than in the exit case. We have identified some intermediate cases where the nanoparticle was sitting at the border with the hydrophilic layer and held by the hydrophilic repulsion.

\section{2 model}

\section{modelling the nanoparticle}

To describe the interaction between the nanoparticle and the monomers we perform the integration of the London-Van der Waals forces between a point-like particle and a dense sphere [Hamaker (1937)].

FigURE 3.2: Sketch representing the nanoparticle radius, $r_{n p}$, and distances from the the bead. $r_{n p}^{2}=r_{i}^{2}+x^{2}-2 r x \cos \theta_{0}$

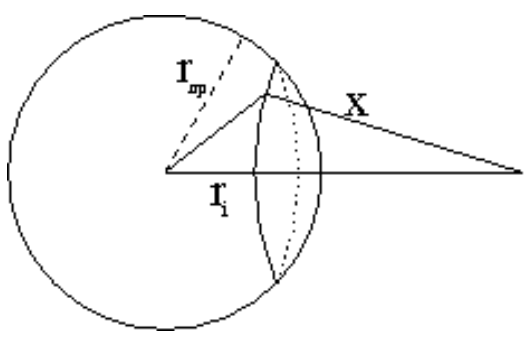

We define $r_{n p}$ as the nanoparticle size, $r_{i}$ is the distance between the center and the bead $i$ and $x$ as the distance between the bead and a nanoparticle volume element. We can see that the surface of nanoparticle at the distance $x$ is:

$$
A\left(x, r_{i} ; r_{n p}\right)=\int_{0}^{2 \pi} \mathrm{d} \phi \int_{0}^{\theta_{0}} \mathrm{~d} \theta x^{2} \sin \theta=\pi \frac{x}{r_{i}}\left(r_{n p}^{2}-\left(x-r_{i}\right)^{2}\right)
$$

The London-Van der Waals forces have an attractive $U_{12} \propto r_{i}^{-12}$ and a repulsive $U_{6} \propto r_{i}^{-6}$ contribution. We integrate the potential over the volume of the nanoparticle.

$$
\begin{aligned}
U_{12}\left(r_{i}\right) & =\pi \int_{r_{i}-r_{n p}}^{r_{i}+r_{n p}} \mathrm{~d} x \frac{1}{r_{i} x^{11}}\left(r_{n p}^{2}-\left(x-r_{i}\right)^{2}\right) \\
U_{6}\left(r_{i}\right) & =\pi \int_{r_{i}-r_{n p}}^{r_{i}+r_{n p}} \mathrm{~d} x \frac{1}{r_{i} x^{5}}\left(r_{n p}^{2}-\left(x-r_{i}\right)^{2}\right)
\end{aligned}
$$

The contributions of the terms $U_{6}$ and $U_{12}$ are:

$$
\begin{aligned}
U_{12}\left(r_{i}\right) & =\frac{\pi}{360 r_{i}}\left(\frac{2 r_{n p}}{\left(r_{i}+r_{n p}\right)^{9}}+\frac{2 r_{n p}}{\left(r_{i}-r_{n p}\right)^{9}}+\frac{1}{\left(r_{i}+r_{n p}\right)^{8}}+\frac{1}{\left(r_{i}-r_{n p}\right)^{8}}\right) \\
U_{6}\left(r_{i}\right) & =\frac{\pi}{12 r_{i}}\left(\frac{2 r_{n p}}{\left(r_{i}+r_{n p}\right)^{3}}+\frac{2 r_{n p}}{\left(r_{i}-r_{n p}\right)^{3}}+\frac{1}{\left(r_{i}+r_{n p}\right)^{2}}+\frac{1}{\left(r_{i}-r_{n p}\right)^{2}}\right)
\end{aligned}
$$

To remove the divergence at $r_{i}=r_{n p}$, where $r_{n p}$ is the conventional radius of the nanoparticle, and for computational reason we join the potential with a linear slope at $U_{12}-U_{6}=20\left[k_{B} T\right]$ and truncate the potential at the cut-off distance $3 r_{n p}$ (see fig 3.2 . The potential is shifted to zero at the cut-off distance, $r_{c}$. 


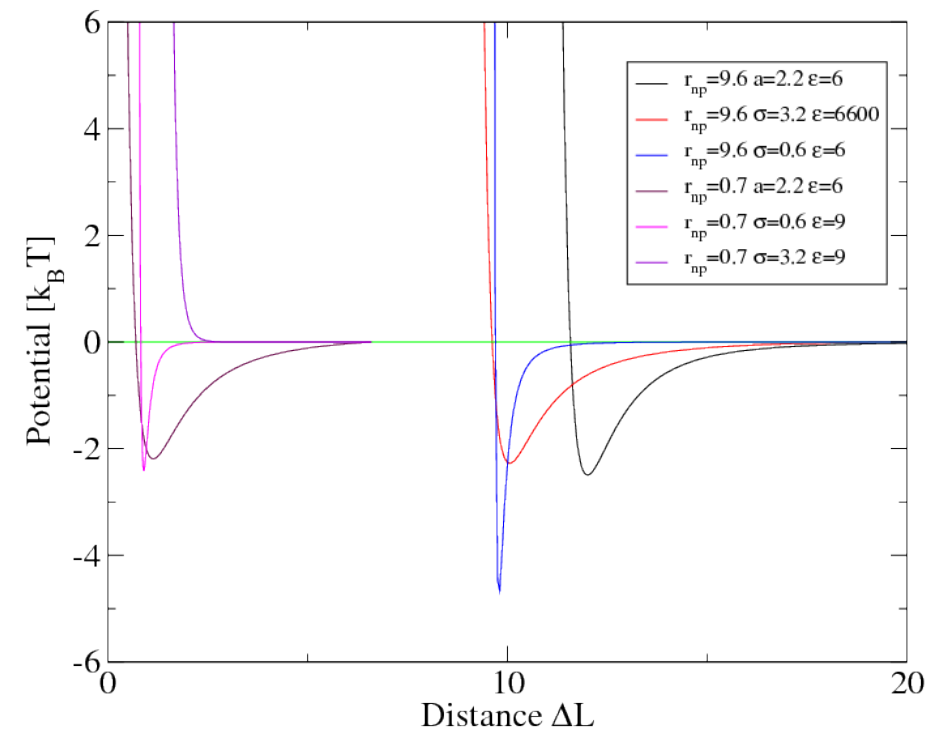

$$
U_{n p}\left(r_{i}\right)=\left\{\begin{array}{ccc}
\epsilon_{c}\left(r_{i}\right) & \text { if } & r<r_{c} \\
0 & \text { if } & r_{i}>3 r_{n p} \\
\epsilon \sigma^{6} U_{12}\left(r_{i}\right)-\epsilon U_{6}\left(r_{i}\right)+\epsilon_{0} & & \text { otherwise }
\end{array}\right.
$$

$\epsilon$ is a prefactor which controls the surface energy of the nanoparticle, $\epsilon_{0}=\sigma^{6} \epsilon U_{12}\left(3 r_{n p}\right)-$ $\epsilon U_{6}\left(3 r_{n p}\right)$ shifts the potential to zero at the cut off $\left(3 r_{n p}\right)$ and $r_{c}$ is the position where the force is $500\left[K_{B} T / \Delta L\right], \epsilon_{c}(r)=U_{n p}\left(r_{c}^{+}\right)-100\left(r-r_{c}\right)$ is a junctions which limits the values of the forces over $100\left[k_{B} T / \Delta L\right]$.

$\sigma$ represents the radius of the small volume elements of the nanoparticle which interacts with the monomers. Some common values of $\sigma$ range between $3-4[\AA]$ but since we are using a mesoscopic description of the nanoparticle we need to set some empirical values for $\sigma$ which do not lead to a too short-ranged (and hence atomistic) potential. We set $\sigma$ close to the cut off length of the monomer-monomer interactions $\sigma \simeq[\Delta L]$.

The potential, 3.3, in the two limiting cases $r_{n p} \rightarrow \infty$ and $r_{n p} \rightarrow 0$ reduces respectively to a 6-12 and 3-9 Lennard-Jones potential.

The input parameters $\epsilon, \sigma$ and $r_{n p}$ of the potential (eq. 3.3 are mapped in the integrated absorbed energy, $\Sigma_{n p}$ and hard core radius of the nanoparticle $r_{h c} . r_{h c}$ is the distance from the center of the nanoparticle to the point where the potential crosses the baseline and $E_{n p}$ is the integral of the negative part of the potential.

$$
\begin{aligned}
r_{h c}\left(\epsilon, \sigma, r_{n p}\right) & =\left\{r \mid U\left(r ; \epsilon, \sigma, r_{n p}\right)=0\right\} \\
E_{n p}\left(\epsilon, \sigma, r_{n p}\right) & =\sum_{i}^{N} U_{n p}\left(r_{n p}, r_{i}\right) \\
& \simeq \rho_{0} \int_{r_{h c}}^{\infty} U\left(r ; \epsilon, \sigma, r_{n p}\right) r^{2} \mathrm{~d} r \mathrm{~d} \cos \theta \mathrm{d} \phi
\end{aligned}
$$

And with $\Sigma_{n p}$ we define the surface energy of the nanoparticle: $\Sigma_{n p}=E_{n p} / 4 \pi r_{n p}^{2}$. The interactions of the nanoparticle with the surrounding monomers give a virial 
contribution to the pressure of the system.

$$
P_{\alpha \beta}^{n c / m}=-\left.\sum_{i, n p} \frac{\partial U}{\partial r}(r)\right|_{r^{i, n p}} \frac{r_{\alpha}^{i, n p} r_{\beta}^{i, n p}}{r^{i, n p}}
$$

Where $\alpha$ and $\beta$ are two Cartesian coordinates and $r^{i, n p}$ is the distance between the monomer $i$ and the nanoparticle.

\section{3 simulation details}

The molecular dynamics simulation scheme is chosen to simulate the system in $N_{c} V T$ and $N_{c} P_{t} T$ ensembles. The simulations are run on a box of $32 \times 32 \times 16\left[\Delta L^{3}\right]$ volume. The bilayer membrane is composed of 2629 chains of 32 beads per chain. The bilayer thickness is measured to be $\bar{d}=4.69[\Delta L]$ and sets the length conversion between experiments and simulations to $3.41[\mathrm{~nm} / \Delta L]$.

The Monte Carlo simulations were run in a box of size of $14 \times 14 \times 14\left[\Delta L^{3}\right]$. The ideal chemical potential is $\mu_{i d}=\ln \frac{N_{c}}{V}=-0.79$ while the excess chemical potential was the one calculated in the previous chapter: $\mu_{e x}=-38.2$.

In the Monte Carlo simulations a hydrophobic nanoparticle sits in the center of the simulation box and has a polymeric coating. This configuration was obtained from an equilibrated molecular dynamics simulation.

\section{4 stability}

In this section we check the stability conditions for the hydrophobic nanoparticle in the bilayer membrane.

Figure 3.4: lhs) Stability diagram of the nanoparticle in function of the nanoparticle radius $r_{n p}$ and the surface energy $E_{n p}$. The vertical lines represent the region of the nanoparticle size where experimentally were observed stable nanoparticles Maskos (2006)]. rhs) Density plot of the membrane around the nanoparticle. The nanoparticle (red circle) sits in the circular hole and is held by the repulsion with the hydrophilic beads.
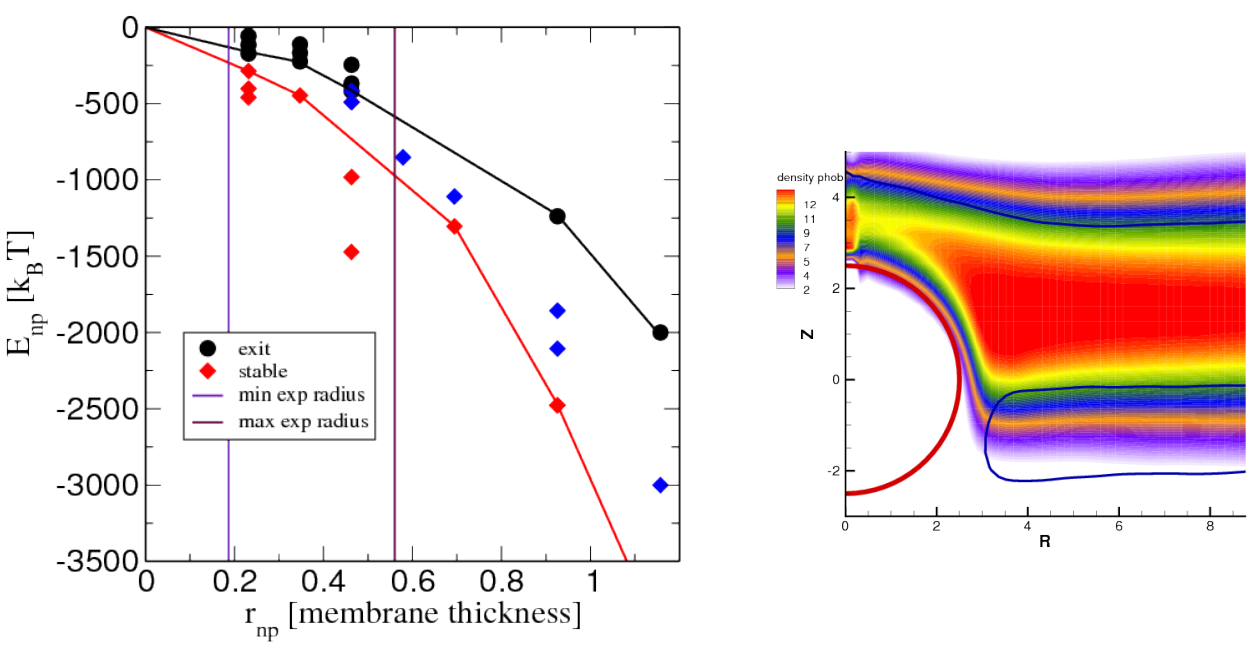

The attractive energy of the nanoparticle to the hydrophobic beads is counterbalanced by the energetic cost of the bending of the leaflets and the rearrangements of the polymers. Initially, we create a cavity inside the bilayer where we position a nanoparticle and wait for a short equilibration time.

We have put nanoparticles of different size and surface energy in the hydrophobic core of the membrane and we have observed which nanoparticles are stable in the bilayer. 
Weak interactive nanoparticles exit the membrane in a short time, (around $13\left[\bar{d}^{2} / D\right]$ ). A stable nanoparticle is held in the membrane for a time longer than three times the average permanence time of a weak nanoparticle.

In the graph (see fig 3.4 ) we have plotted the condition of stability of the nanoparticle depending on its size and surface energy. The region of stability and instability are clearly identified by the black and the red lines. These lines (see fig 3.4 ) suggests a linear relationship between the nanoparticle surface and the surface energy. The larger is the nanoparticle the stronger has to be the interaction towards the hydrophobic beads for the stability.

The blue dots contained within the red and black lines (in fig 3.4 refer to some intermediate cases where the nanoparticle was held by the repulsion with the hydrophilic beads.

\section{5 chemical potential of the nanoparticle}

In case of a stable nanoparticles it is not clear whether the favourable condition for a nanoparticle would be to stay embedded in the membrane or to exit from the membrane with a polymer coating. The energy barrier of this process is too large to observe spontaneously such as event during a molecular dynamics simulation.

To solve this doubt we have decided to calculate and compare the chemical potentials of the nanoparticle in the two configurations.

The chemical potential is the energy of inserting one nanoparticle into the system. To perform this calculation we define one parameter $\lambda$ that shuts off the interactions between the nanoparticle and the polymers. The chemical potential of the nanoparticle is obtained from:

$$
\mu_{n p}=\int_{0}^{1}<\partial_{\lambda} U_{n p}>_{N P_{t} T} \mathrm{~d} \lambda \quad U_{n p}:=\sum_{i}^{N} \lambda U_{n p}\left(r_{i}\right)
$$

We have chosen twenty different values of $\lambda$ between 0 and 1 and calculated the average interaction energy between the nanoparticle and the polymer in the membrane. The simulation refers to a nanoparticle of $r_{n p}=0.46[\bar{d}]$ and $E_{n p}=-1436\left[k_{B} T\right]$ and we can extract the exchange energy between the nanoparticle and the bilayer (see fig 3.5 ).
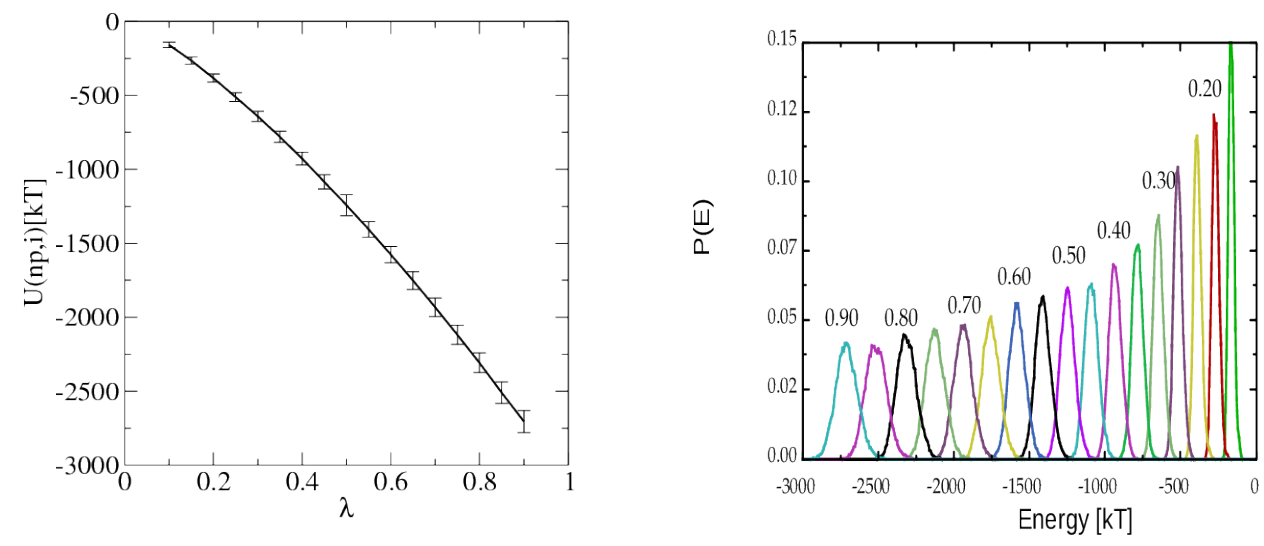

We can see in the picture (see fig 3.5 that the interaction energy between the nanoparticle and the polymers smoothly decreases decreasing $\lambda$. The histograms of the energy distributions nicely overlap as required for the weighted histogram analysis Kumar et al. (1992); Bartels (2000)].
$D$ polymer diffusivity (see table $4.4,\left[\bar{d}^{2} / D\right]$ is the time required for a lipid to diffuse of one unit of bilayer thickness.
Figure 3.5: lhs) Average ensemble of the energy between the nanoparticle and the polymer in the bilayer depending on the shutting off parameter $\lambda$. rhs) single histograms for different values of $\lambda$, all the histograms overlap with their neighbours. 
Figure 3.6: lhs) Average ensemble of the energy between the nanoparticle and the coating polymers depending on the shutting off parameter $\lambda$ in Monte Carlo simulations. rhs) single histograms for different values of $\lambda$, all the histograms overlap with their neighbours.
To calculate the chemical potential of the coated nanoparticle we have to impose the condition that the polymers of the coating have the same chemical potential of the polymers in the bilayer. For this reason we run Monte Carlo grand canonical simulations where we can control the chemical potential of the polymers. The chemical potential was calculated in the previous chapter from a sequence of molecular dynamics simulations of a stable tensionless bilayer.
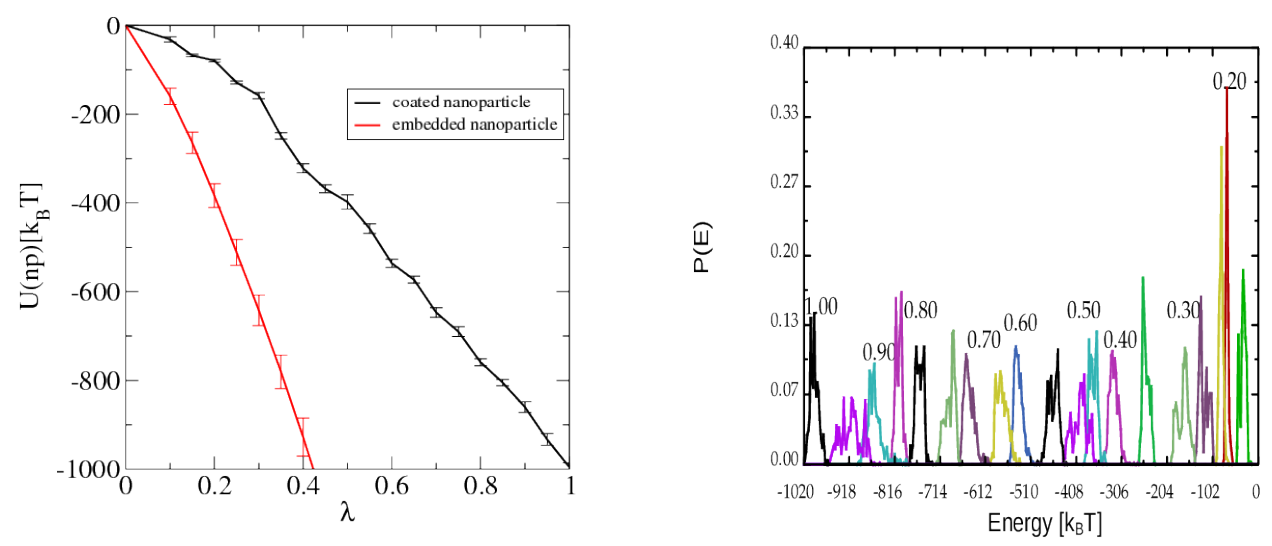

In the Monte Carlo simulations the number of coating polymers fluctuates around a mean value that depends on the parameter $\lambda$. The difference of the mean number of polymers varies between 20 and 160 , for $\lambda<0.3$ the nanoparticle is not completed coated. Looking at the graph (fig 3.6 ) the sample of the interaction energies is not satisfactory to perform the weighted histogram analysis and calculate the chemical potential of the nanoparticle. The two curves in the graph (3.6) show nevertheless a strong difference between the interaction energies of the embedded and the coated nanoparticle and allow us to state that this particular nanoparticle is energetically more favourable inside the membrane.

\section{6 conclusions}

In this chapter we have developed a method to study the stability of nanoparticles inside the bilayer. We have distinguished between weak nanoparticles that exit the bilayer in a short time and stable nanoparticles. During the limited simulation time of the molecular dynamics simulations we cannot state whether the nanoparticle is energetically more stable outside the bilayer with a polymeric coating. We have chosen one particular stable nanoparticle (described by its radius, $r_{n p}$, and surface energy $E_{n p}$ ) to develop a method to state the energetic difference of the two configurations. We have studied the interaction energy between the nanoparticle and the polymers in the both configurations and found that the particular nanoparticle chosen $\left(r_{n p}=0.46[\bar{d}]\right.$ $\left.E_{n p}=-1463\left[k_{B} T\right]\right)$ is more stable inside the bilayer.

Comparing to the experimental observations, stable nanoparticles were observed to have a diameter in the range of $3-9[\mathrm{~nm}]$ [Maskos (2006)]. Even though we have found no data about the experimental surface energy of the nanoparticle, we refer to the stability diagram (see fig 3.4 to estimate that the surface energy should range between $20-100\left[k_{B} T\right]$. 


\section{Chapter 4}

\section{mechanical and phase properties of planar membranes}

The model depends on a set of input parameters that modify the mechanical properties and phase behaviour of the membrane. We refer to the values written in the table below (our reference model) and study the dependence of the mechanical properties of self-assembled bilayers on these parameters.

$$
\begin{aligned}
& \rho_{\text {coex }}=6\left[N_{c} / \Delta L^{3}\right] \quad \chi N=30 \quad k N=70 \\
& N_{b}=10 \quad N_{p h o b}=8 \quad R_{e}=2[\Delta L] \\
& k_{s p}=20.537 \quad k_{b}=3 \quad v_{B B}=-0.1
\end{aligned}
$$

TABLE 4.1: Reference set for the parameters used in the simulations

To reproduce the behavior of membranes under biological condition we explore the phase space to identify the range of the input parameters where the lipids self-assemble in a planar fluid bilayer. The change in the chain architecture conditions the phase transition from the lamellar to the inverted hexagonal phase. We choose to change the compressibility to move the lipid bilayer between the fluid/gel phase transition.
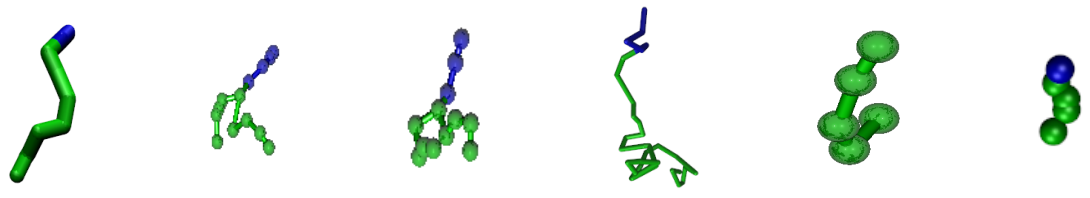

FIGURE 4.1: Sketch of the molecules studied with this model: single tail lipid [8-10], PC lipid [9-13], PE lipid [9-12], flexible polymer [27-32], oil [6], cholesterol [5-1], where the brackets indicate $\left[N_{p h o b}, N_{b}\right]$.

To set a time and length scale for the self-assembled system, we calculate the lipid diffusivity and the membrane thickness and compare the obtained values to the ones known in the literature. Thanks to the softness of the model, we can successively calculate different free energy contributions from thermodynamics fluctuations and obtain the values of the unperturbed system. These values are the reference values to quantify the local membrane perturbation around the inclusion. 


\section{1 lipid composition}

The lipid composition we study are lipid chains composed by one block of hydrophobic beads and one block of hydrophilic beads.

If not further specified, we refer to a lipid with a single tail where the hydrophobic block has 8 beads and the hydrophilic 2 . Referring to two tails lipid we distinguish between PE and PC lipids. The PE lipids have 9 hydrophobic beads and 3 hydrophilic, the PC lipids 9 hydrophobic and 4 hydrophilic. Oil is represented by short chains composed by 4 hydrophobic beads, and cholesterol has an additional hydrophilic bead at the top.

\section{2 exploring the phase space}

The parameters that mainly control the morphology of the self-assembly structure are the incompatibility and the asymmetry of the chains.

Figure 4.2: Lipid architectures: lhs) [9-10], middle) [9-12] double tailed, rhs) [9-13] double tailed corresponding to the phases: lhs) inverted micelle, middle) inverted hexagonal, rhs) lamellar. In the middle picture, elongated linear stalks connect the membranes of the stack and indicates an inverted hexagonal phase. The relaxation into an inverted hexagonal phase is imitated by the geometrical constrictions of the simulation box.

Figure 4.3: Top view representation of the chain conformation in: lhs) non interdigitated gel phase (only the lower leaflet is shown), middle) interdigitated gel phase, rhs) and fluid phase. On the left hand side the color legend is shown.

TABle 4.2: Color representation of the chain, every color represents a different chain conformation.

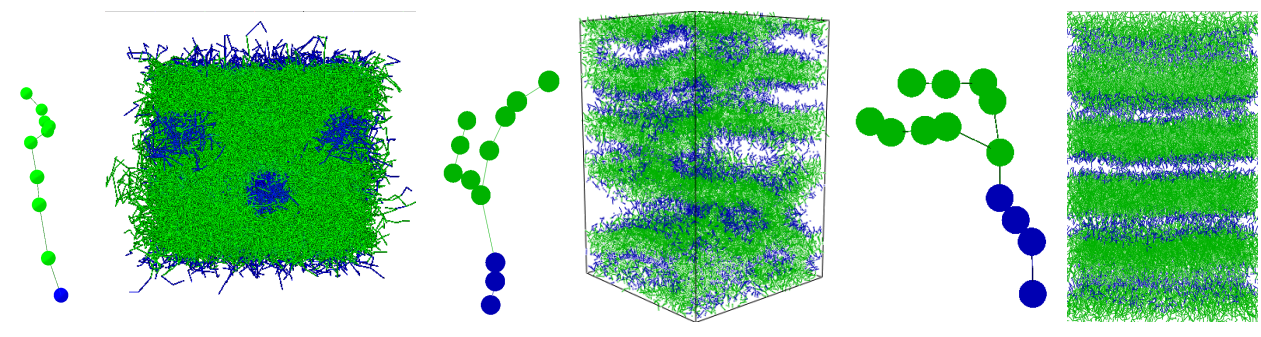

By changing the architecture of the chains we reproduce the micellar, lamellar and inverted hexagonal phases [Cullis et al. (1986); Israelachvili (1998)].

A lipid membrane has a rich phase behaviour which deeply influences the mechanical properties of the self-assembled structure. Changing the compressibility $k N_{b}$ and the lateral pressure, $P_{l}$, we have identified different phases and found the parameter range corresponding to the fluid state.
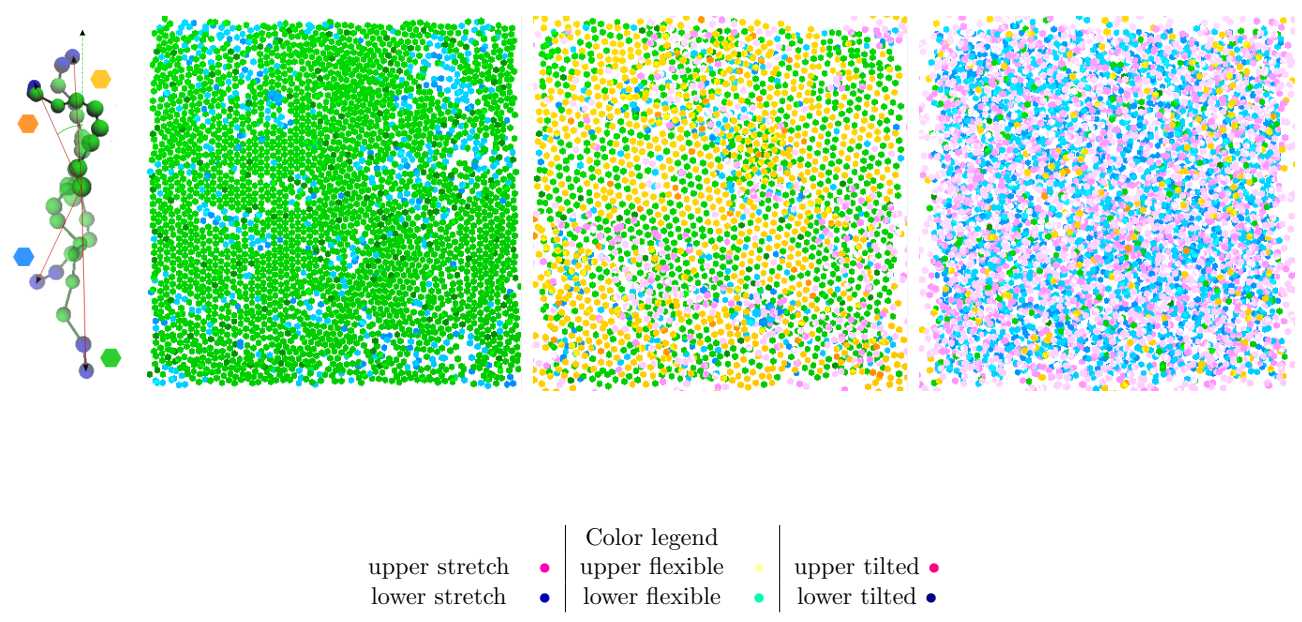

Varying the density consistence, $\rho_{\text {coex }}$, the compressibility, $k_{N}$, and the lateral tension, $P_{l}$, we can represent the rich phase behavior of lipid bilayers. The phases shown in the picture (see fig 4.3 are: $\left.L_{\beta}\right)$ non-interdigitated gel phase $\left(\rho_{\text {coex }}=5, P_{l}=0.28\right)$, $L_{\beta I}$ interdigitated gel phase $\left.\left(\rho_{\text {coex }}=5, P_{l}=0.40\right), L_{\alpha}\right)$ fluid $\left(\rho_{\text {coex }}=6, P_{l}=0\right)$. We 
define a stretched lipid when:

$$
\sum_{i}^{N_{b}} \frac{\left|z_{i, i+1}\right|}{r_{i, i+1}}<0.7
$$

and flexible otherwise. The tilted chains are the chains whose orientation is smaller than $60^{\circ}$ with respect to the bilayer normal.

\section{3 thickness}

The thickness $\bar{d}$ is defined as the width at the half height of the hydrophobic density profile.
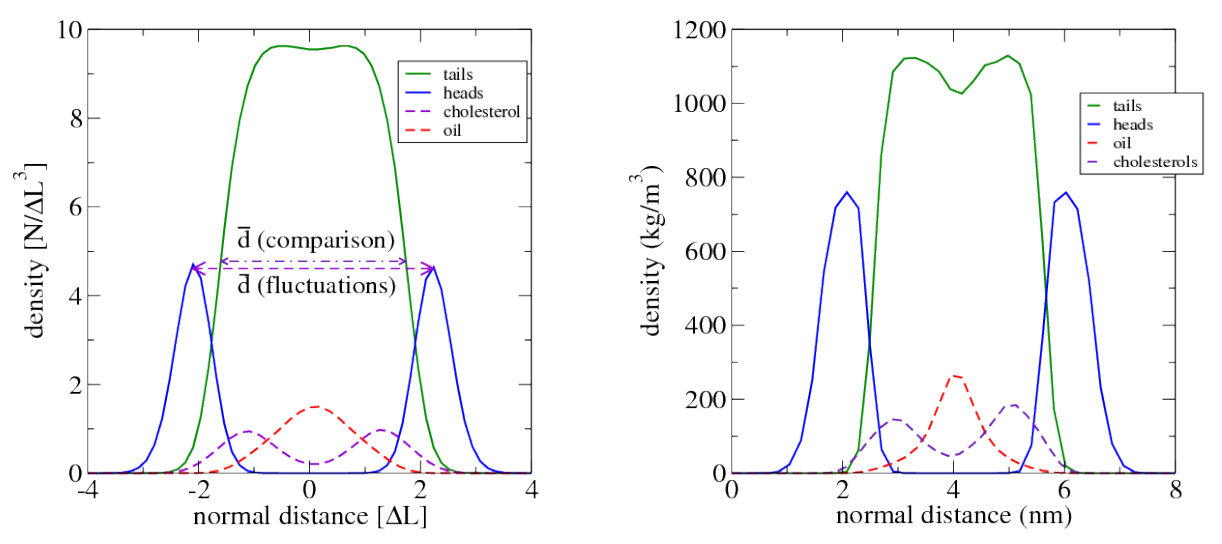

Figure 4.4: Density profile for the hydrophobic (green) and hydrophilic (blue) monomers and the definition of the thickness for the soft coarse-grained model (lhs) and the Martini force field. In the plots the density of tails and heads refer to the homogeneous membrane, the density of oil and cholesterol is drawn in the same picture only for the spatial reference.

Since the membrane is fluctuating the total density profile is the average of the single contributions of small patches of area $\left[4 \Delta L^{2}\right]$. The patches are chosen to be approximately planar and their density profiles are summed, shifting the center of mass to the same reference point.

The oil sits on the equatorial plane of the membrane, while the cholesterol sits at the interface (see fig 4.4). The density calculated for the soft coarse-grained model is much less accurate than a coarse-grained simulation with the Martini force field calculated with the same method (see fig 4.4 due to the simplification of the interactions and lipid constituents.

Out of the Martini simulations we measure the width at half maximum of density of the hydrophobic beads and measure $3.03[\mathrm{~nm}]$. Using the same definition of the bilayer thickness we parametrise the length of our model: $1[\Delta L]=1.086[\mathrm{~nm}]$. We refer to the comprehensive work of [Kučerka et al. (2011)] for comparing different definitions of the membrane thickness from the analysis of small-angle neutron and Xray scattering data. In the article the hydrocarbonate region thickness is $2.9[\mathrm{~nm}]$ thick (POPC bilayer @303[K], $\bar{d}=2.6[\mathrm{~nm}]$ for a DMPC bilayer at the same temperature) and it is described as reference thickness where the function of an integral membrane protein is affected by hydrophobic mismatch.

\section{4 time scale}

The simulation time is compared with the experimental time mapping the value of the lipid's diffusivity onto the known values of the literature. Atomistic and systematically coarse-grained simulation models are capable of reproducing the experimentally observed lipid diffusivity Apajalahti et al. (2010b); Falck et al. (2008)]. 
Figure 4.5: Dependence of the mean square displacement on the input parameters $k N_{b}$.

$D$ : Diffusion coefficient, $\operatorname{msd}(t)$ : mean square displacement

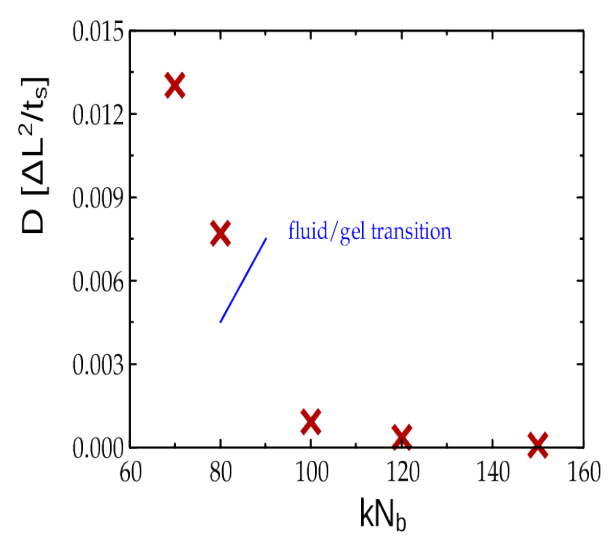

The diffusion coefficient, $D$, is defined as the asymptotic limit of the lateral mean square displacement, $\operatorname{msd}(t)$, divided by the total time $t$ :

$$
D=\lim _{t \rightarrow \infty} \frac{\operatorname{msd}(t)}{4 t} \quad \operatorname{msd}(t):=\left\langle(\mathbf{x}(t)-\mathbf{x}(0))^{2}\right\rangle
$$

To improve the statistics, for every time, $t$, we average all the time differences with the same duration:

$$
x(j \Delta t)-x(0)=\frac{\sum_{i}}{N_{i}}(x((i+j) \Delta t)-x(i \Delta t))
$$

This average can deviate from the single time differences if the system is not equilibrated or perturbed since it depends only on time interval differences. To calculate the diffusion coefficient we plot the mean square displacement in the axis $(1 / t, \operatorname{msd}(1 / t) / t)$ and extrapolate the value for $\operatorname{msd}(0)$. We also calculate the diffusion coefficient from the slope of the msd versus time in the diffusive regime.

For $k N_{b}>80$ the membrane is in a gel phase [Hömberg and Müller (2010)] and the diffusivity decreases significantly. The simulation time, $t_{s}$, is the internal time used in the simulation and it is connected with the integration time step $\Delta t_{s}=0.005\left[t_{s}\right]$. The current simulation time is $t=N_{s} \Delta t_{s}\left[t_{s}\right]$ where $N_{s}$ is the current number of steps. To set the time scale of the simulations we compare the diffusivity calculated above with the diffusivity calculated in experiments. There is a rich literature about experimental values of lipid diffusivity obtained with different methods: pulsed NMR of multilamellar POPC liposome $\left(D=8.6 \pm 0.2\left[\mu \mathrm{m}^{2} / \mathrm{s}\right]\right.$ Gaede and Garwrisch (2003)]) using fluorescence correlation spectroscopy (FCS) on POPC bilayers $\left(D=7.3 \pm 0.25\left[\mu \mathrm{m}^{2} / \mathrm{s}\right]\right.$ Kyong and Sheets (2008)]), dual focus FCS on POPC/POPE mixtures $\left(D=12 \pm 2.5 \cdot\left[\mu \mathrm{m}^{2} / \mathrm{s}\right][\right.$ Weiß and Enderlein (2012)] $)$, and using florescence recovery after photobleaching (FRAP) $\left(D=8 \pm 4\left[\mu m^{2} / s\right][\right.$ Kocun et al. (2011)]). We choose to use the third value because does not refer to a supported bilayer (where the diffusivity is influenced by the presence of the support) and obtain: $1\left[t_{s}\right]=0.93[n s]$. The time definition is used to define the relaxation time of the lipids inside the membrane. The relaxation time is a useful information to decide how long to simulate before writing on a file the system configuration. For an efficient occupation of the memory, the system configurations should be separated by some relaxation time units to avoid too strong correlation between the subsequent snapshots. To efficiently use the simulation time, the time between consecutive snapshots can be chosen around some tens of relaxation time units, this allows to have weak correlated configurations for the calculations of the ensemble averages. 


\section{5 thickness, lateral area, and density fluctuations}

In this section we calculate the coupling modulus of the fluctuation of the thickness, lateral area and density for a planar membrane.
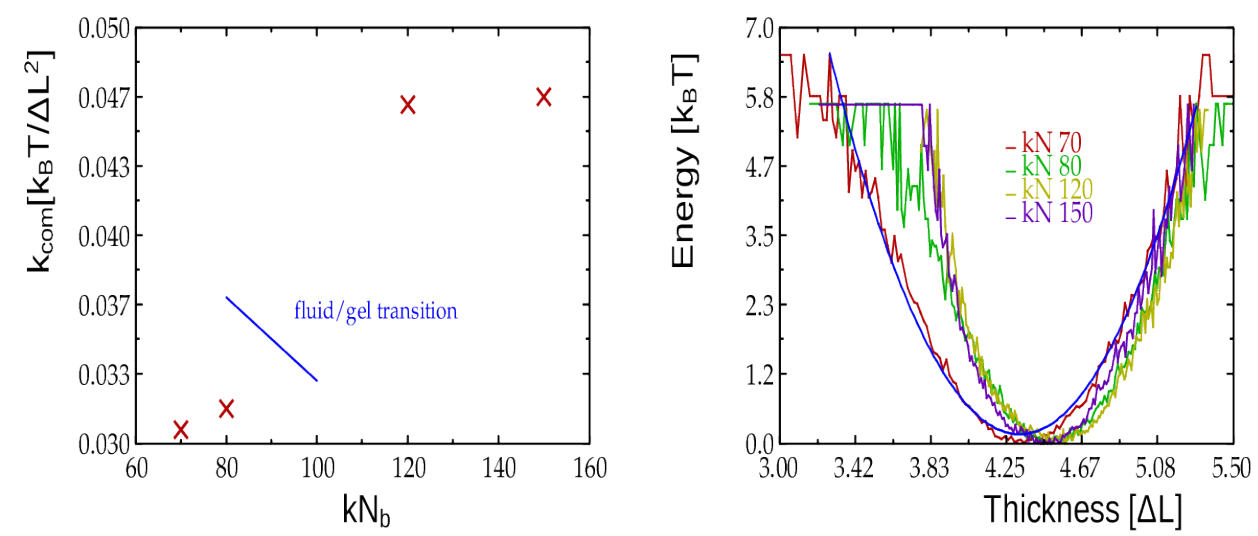

Thanks to the softness of the model we calculate these free-energy contributions from the thermal fluctuations using the linear response theorem which relates the fluctuations of a system around its equilibrium state to its response to weak perturbations Plischke and Bergensen (1994)].

We consider one quantity $s_{L}$ which can be: the thickness of the membrane, $d_{L}$, the number of chains per area, $\sigma_{L}=n_{L} / L^{2}$, or the local density, $\rho_{L}=n_{L} / \sigma_{L} d_{L}$. Each quantity is defined on a portion of the membrane, that we call patch, with area $L \times L$. We define a coupling modulus, $k_{s}$, which can be: the elastic coupling, $k_{e l}$, the area compressibility, $k_{\text {com }}$, or the density compressibility, $k_{\text {melt }}$. The coupling modulus $k_{s}$ controls the amplitude fluctuations of the quantity $s_{L}$.

We express the free energy around the value $s_{L}$ expanding the isothermal free energy in a Taylor series in powers of $\alpha=\Delta s_{L} / \bar{s}_{L}$ Daily and Elson (1984)]:

$$
F_{a_{l}}-F_{a_{l}}^{0}=\left(\frac{\partial F}{\partial \alpha}\right)_{T} \alpha+\left(\frac{\partial^{2} F}{\partial \alpha^{2}}\right)_{T} \frac{\alpha^{2}}{2}+\ldots
$$

In equilibrated systems $\langle s\rangle=\bar{s}$ and therefore the linear term vanishes. We express the free energy difference in:

$$
\Delta F_{s}=\int \mathrm{d} x \mathrm{~d} y \frac{k_{s}}{2} \frac{(s(x, y)-\bar{s})^{2}}{\bar{s}^{2}}+O\left((s-\bar{s})^{4}\right)
$$

To determine the coupling modulus, which controls the amplitude of the fluctuation of $s$ around the mean value $\bar{s}$, we calculate the statistical fluctuation of the quantity $s$ depending on the size $L \times L$ of the patch analyzed [Müller and Schick (1996)]. In thermodynamics the fluctuations of an extensive quantity scale like the inverse of the number of chains:

$$
\Delta s_{L}^{2}=\frac{\left\langle s_{L}^{2}\right\rangle-\left\langle s_{L}\right\rangle^{2}}{N_{c}}=\bar{\sigma}_{c} \frac{\left\langle s_{L}^{2}\right\rangle-\left\langle s_{L}\right\rangle^{2}}{L^{2}} \quad N_{c}=\bar{\sigma}_{c} L^{2}
$$

The coupling modulus is the limit for infinite size patches of the fluctuations of $s$.

$$
\frac{1}{k_{s}}=\lim _{L \rightarrow \infty} \frac{L^{2}\left\langle\Delta s^{2}\right\rangle_{L}}{\bar{s}^{2}}
$$

To calculate the coupling modulus we plot the $L^{2}<\Delta s^{2}>_{L} / \bar{s}^{2}$ versus the inverse of the patch area and extrapolate the value of the curve for a zero value of the abscissa
FiguRE 4.6: lhs) Compressibility for different values of the input parameter $k N_{b}$. rhs) Harmonic approximation derived from the ensemble distribution of the membrane thickness for a patch size of $3 \times 3\left[\Delta L^{2}\right]$.

$\Delta s_{L}=\left(s_{L}-\bar{s}\right), \alpha=\Delta s_{L} / \bar{s}, L$ lateral dimension of the patch considered, $d_{L}$ thickness of the patch, $n_{L}=\sigma_{L} L^{2}$ number of chain in the patch, $\rho_{L}=n_{L} / d_{L}$ density of lipids in the patch. 
Figure 4.7: Standard deviation versus patch area, $\left(\langle\Delta s\rangle, L^{2}\right),(\mathrm{lhs})$, fluctuation versus inverse patch area, $\left(\frac{L^{2}\langle\Delta s\rangle}{\bar{s}^{2}}, L^{-2}\right),(\mathrm{rhs})$.
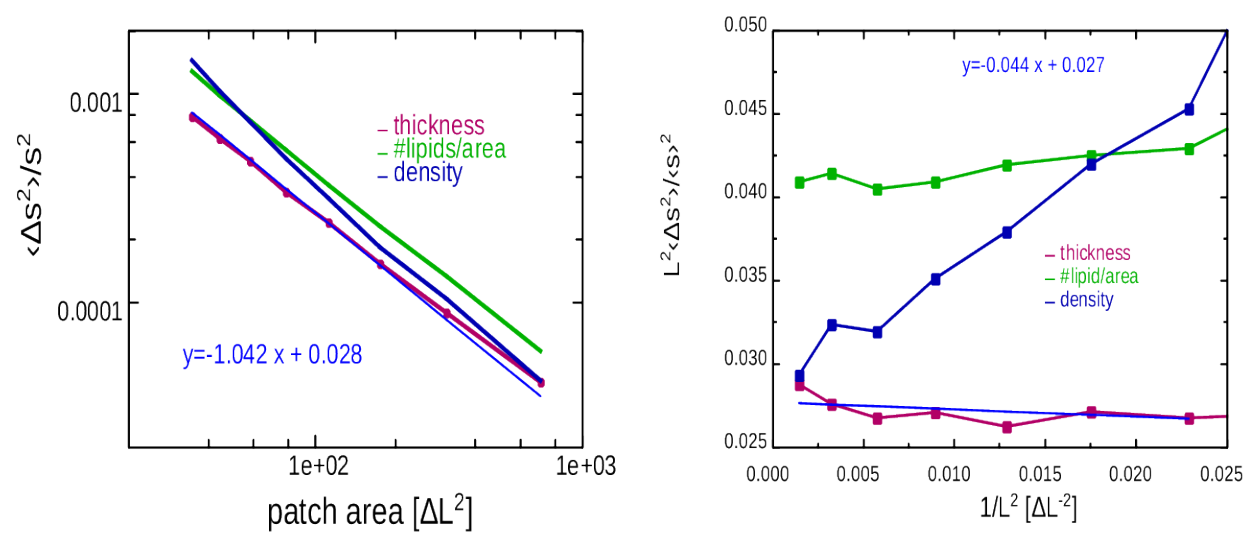

(see fig 4.7). The first point on the left hand side of the graph (see fig 4.7) represents a patch which spans almost all the simulation area. In this case the fluctuations of the number of lipids (which affects the area and density compressibility) are limited by the fixed amount of lipids. We exclude this point from the analysis.

\section{6 energetic contributions}

The free energy for the fluctuations of the thickness, the lateral area, and the density fluctuation is:

$$
\Delta F_{\text {fluct }}=\int \mathrm{d} x \mathrm{~d} y\left(\frac{k_{e l}}{2} \frac{(d-\bar{d})^{2}}{\bar{d}^{2}}+\frac{k_{\text {melt }}}{2} \frac{(\rho-\bar{\rho})^{2}}{\bar{\rho}^{2}}\right)
$$

We have excluded the area compressibility term since the variables are not independent: $\sigma=d \rho$. The increase or decrease of the thickness of the membrane causes a stretching or compression of the lipids which involves a change in the lateral area. Since the density is not independent from the thickness we propagate the fluctuations of the $V=A d, d \rho=\sigma$ independent variables:

$$
\left\langle\Delta \sigma^{2}\right\rangle=\left\langle\Delta(\rho d)^{2}\right\rangle=\left(\frac{\partial \sigma}{\partial d}\right)^{2}\left\langle\Delta d^{2}\right\rangle+\left(\frac{\partial \sigma}{\partial \rho}\right)^{2}\left\langle\Delta \rho^{2}\right\rangle=\bar{\rho}^{2}\left\langle\Delta d^{2}\right\rangle+\bar{d}^{2}\left\langle\Delta \sigma^{2}\right\rangle
$$

Dividing the last equation by $\bar{\sigma}^{2}$ we obtain:

$$
\frac{\left\langle\Delta \sigma^{2}\right\rangle}{\bar{\sigma}^{2}}=\frac{\left\langle\Delta \rho^{2}\right\rangle}{\bar{\rho}^{2}}+\frac{\left\langle\Delta d^{2}\right\rangle}{\bar{d}^{2}}
$$

This equation sets a relation between the coupling moduli:

$$
\frac{1}{k_{\text {com }}}=\frac{1}{k_{\text {melt }}}+\frac{1}{k_{e l}}
$$

We see in the graph (see fig 4.7) that this relation is satisfied within $25 \%$. The difference arises from the head groups which additionally restrict area fluctuations.

To express the free energy in terms of thickness and area per lipid density we substitute $\rho=\sigma / d$ and we expand in series of $(d-\bar{d})$ obtaining:

$$
\frac{(\rho-\bar{\rho})^{2}}{2 \bar{\rho}^{2}}=\frac{(\sigma-\bar{\sigma})^{2}}{2 \bar{\sigma}^{2}}+\frac{3 \sigma^{2}-2 \sigma \bar{\sigma}}{\bar{\sigma}^{2}} \frac{(d-\bar{d})^{2}}{2 \bar{d}^{2}}+O\left((d-\bar{d})^{4}\right)
$$

where we have have considered zero the averages of the quantities $(d-\bar{d})$. 


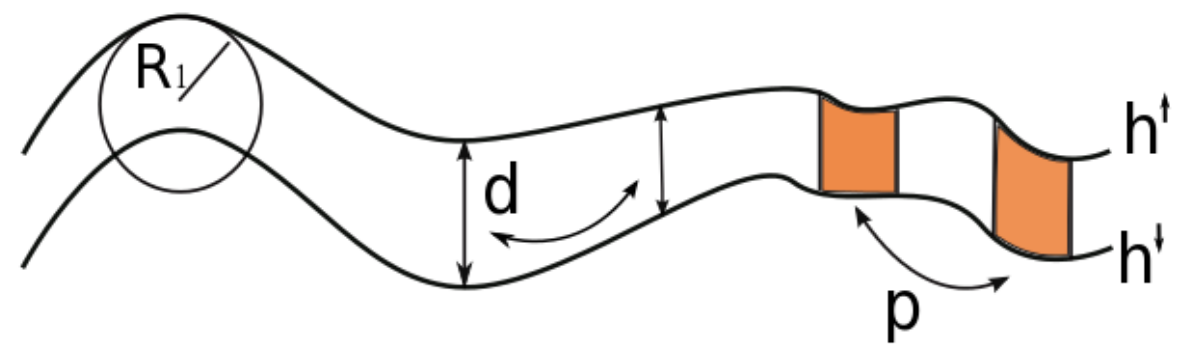

The most relevant energetic contributions for our purpose are summarised in the following free-energy expression:

$$
F=\frac{1}{2} \int \mathrm{d} x \mathrm{~d} y\left(k_{\text {ben }}\left(\frac{1}{R_{1}}+\frac{1}{R_{2}}\right)^{2}+k_{\text {melt }} \frac{(\rho-\bar{\rho})^{2}}{\bar{\rho}^{2}}+k_{\text {com }} \frac{(a-\bar{a})^{2}}{\bar{a}^{2}}\right)
$$

The first term of the free energy is the bending energy calculated by integrating the local curvatures and has modulus $k_{\text {ben }}$ Helfrich [1978)]. The energetic contribution of the area compressibility expressed in the $N V T$ and $N P_{l} T$ ensemble are:

$$
F_{N V T}=\frac{k_{c o m}}{2}(a-\bar{a})^{2} \quad F_{N P_{l} T}=F_{N V T}-A \gamma \quad \gamma:=\frac{\partial F_{N V T}}{\partial A}
$$

The pressure in this case is $\gamma=k_{\text {com }}(a-\bar{a}) / \bar{a}$. For linear changes in the lateral area the modulus $\gamma$ controls the stretching energy of the membrane. For tensionless membrane (most of the conditions studied) this energy is zero.

\section{Monge representation}

For small deformations of the membrane we can describe the membrane as a unique function, $h(x, y)$, (Monge representation) where every point on the plane $(x, y)$ is assigned a unique membrane height. Following the analysis in Farago and Pincus (2004)] way we can transform the membrane height in Fourier space:

$$
h_{\boldsymbol{q}}=\int \mathrm{d} r h(\boldsymbol{r}) e^{-\imath \boldsymbol{r} \boldsymbol{q}} \quad h(\boldsymbol{r})=\frac{1}{2 \pi} \int \mathrm{d} q h_{\boldsymbol{q}} e^{\imath \boldsymbol{r} \boldsymbol{q}}
$$

The total area of the membrane is:

$$
A=\int \mathrm{d} x \mathrm{~d} y \sqrt{1+(\nabla h)^{2}} \quad A=L^{2}+\int \mathrm{d} q q^{2} h_{\boldsymbol{q}} h_{-\boldsymbol{q}}+O\left(\left|h_{q}\right|^{4}\right)
$$

To total Gaussian curvature is:

$$
J=\int \mathrm{d} x \mathrm{~d} y\left(\nabla^{2} h\right)^{2} \quad J=\int \mathrm{d} q q^{4} h_{\boldsymbol{q}} h_{-\boldsymbol{q}}+O\left(\left|h_{\boldsymbol{q}}\right|^{4}\right)
$$

The expression of the Hamiltonian in the Monge representation is:

$$
\begin{aligned}
& F(h)=F(h=0)+\frac{1}{2} \int \mathrm{d} x \mathrm{~d} y\left(k_{b e n}\left(\nabla^{2} h\right)^{2}+\gamma(\nabla h)^{2}+k_{e l} d^{2}\right) \\
& F(h)=F(h=0)+\int \mathrm{d} q\left(\left(k_{b e n} q^{4}+\gamma q^{2}\right) h_{\boldsymbol{q}} h_{-\boldsymbol{q}}+k_{e l} d_{\boldsymbol{q}} d_{-\boldsymbol{q}}\right)
\end{aligned}
$$

The equation 4.14 recalls the definition in the Monge representation of the equation (4.19).

The thickness of the membrane is defined as:

$$
d(\boldsymbol{r}):=h_{\text {up }}(\boldsymbol{r})-h_{\text {down }}(\boldsymbol{r})
$$

FIGURE 4.8: Sketch of the continuum description of a lipid membrane. $a=1 / \sigma,(\sigma-\bar{\sigma})=-(a-\bar{a}) / \bar{a}^{2}, \gamma$ isotropic tension, $R_{1}, R_{2}$ two orthogonal principal curvatures.
$L$ box size, $N_{s}$ number of samples, $\sqrt{1+(\nabla h)^{2}} \simeq 1+(\nabla h)^{2}$. $h_{u p}$ height of the upper monolayer, $h_{\text {down }}$ height of the lower monolayer. 
$j_{\boldsymbol{q}}$ are Lagrange multipliers to We calculate the partition function from the surface Hamiltonian $H(h(\boldsymbol{r}))$ : enforce $\overline{h_{\boldsymbol{q}}}=\left\langle h_{\boldsymbol{q}}\right\rangle$

$$
Z=\int D\left[\left\{h_{\boldsymbol{q}}\right\}\right] e^{-H\left(\left\{j_{\boldsymbol{q}}\right\}\right)-\sum_{\boldsymbol{q}} h_{\boldsymbol{q}} j_{\boldsymbol{q}}}
$$

$G$ is the Gibbs free-energy:

The mean value and the standard deviation of the height fluctuation are now:

$$
\overline{h_{\boldsymbol{q}}}=\left\langle h_{\boldsymbol{q}}\right\rangle=-\frac{\partial G}{\partial j_{\boldsymbol{q}}} \quad\left\langle h_{\boldsymbol{q}} h_{-\boldsymbol{q}}\right\rangle-\left\langle h_{\boldsymbol{q}}\right\rangle\left\langle h_{-\boldsymbol{q}}\right\rangle=-\frac{\partial^{2} G}{\partial j_{\boldsymbol{q}} \partial j_{-\boldsymbol{q}}}
$$

$\frac{\partial F}{\partial h_{q}}=j_{q}$ We relate the Helmoltz free energy to the Gibbs free energy via:

$$
F\left(\left\{h_{\boldsymbol{q}}\right\}\right)=G\left(\left\{j_{\boldsymbol{q}}\right\}\right)+\sum_{\boldsymbol{q}}\left\langle h_{\boldsymbol{q}}\right\rangle j_{\boldsymbol{q}}
$$

Recalling the former expression of the free energy (eq.4.19) we can explicitly calculate $j_{q}$ :

$$
j_{\boldsymbol{q}}=L^{2}\left(\gamma q^{2}+k_{\text {ben }} q^{4}+O\left(q^{6}\right)\right)\left\langle h_{\boldsymbol{q}}\right\rangle
$$

Substituting 4.24 into 4.23:

$$
G \simeq F\left(\left\{\left\langle h_{\boldsymbol{q}}\right\rangle\right\}=\{0\}\right)-\sum_{\boldsymbol{q}} \frac{j_{\boldsymbol{q}} j_{-\boldsymbol{q}}}{2 L^{2}\left(\gamma q^{2}+k_{\text {ben }} q^{4}\right)}
$$

Considering 4.22 we can finally write the power spectrum of the height fluctuation:

$$
\left\langle h_{\boldsymbol{q}} h_{-\boldsymbol{q}}\right\rangle=\left\langle\left|h_{\boldsymbol{q}}\right|^{2}\right\rangle=\frac{1}{L^{2}\left(\gamma q^{2}+k_{b e n} q^{4}\right)}
$$

While the power spectrum of the thickness fluctuation (for long wavelength motion) is:

$$
<d(\boldsymbol{q}) d(-\boldsymbol{q})>=\frac{1}{L^{2} k_{e l}}
$$

Figure 4.9: lhs) Monge representation of the bilayer: height (top) and thickness fluctuations (bottom), the color map represent the height (top) and the thickness (bottom). rhs) Power spectrum of the local height of the membrane $\left\langle h(q)^{2}\right\rangle$ versus the reciprocal squared space, $q^{2}$.
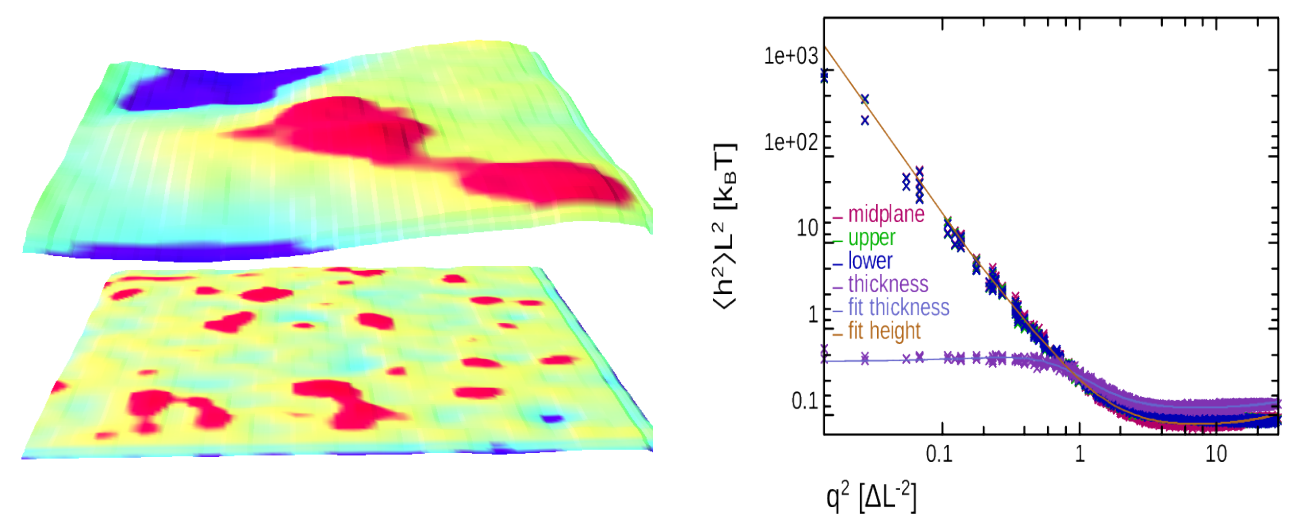

A more detailed model for the energetic contributions considers the membrane composed by two coupled monolayers instead of a single sheet [West et al. (2009); Branningan and Brown (2007); Seifert (1997); Szleifer et al. (1990)]. The bending of the membrane cause the compression of one monolayer and the extension of the opposed one and other energetic contributions have to be considered. The neutral surface of a monolayer, $\left(h^{\uparrow}(x, y), h^{\downarrow}(x, y)\right)$, is defined by the property that bending and stretching are decoupled in energy when both deformations are defined with respect to it and it is distant $\bar{d} / 2$ from the bilayer midplane Seifert (1997)]. We define the midplane and the thickness fluctuations:

$$
h=\frac{h^{\uparrow}+h^{\downarrow}}{2} \quad d=h^{\uparrow}-h^{\downarrow}+\bar{d}
$$


The total energy per molecule of a symmetric bilayer is Branningan and Brown (2007)]:

$$
f_{h}=\frac{k_{b e n}}{2}\left(\nabla^{2} h\right)^{2}+\frac{k_{c o m}}{\bar{d}^{2}} d^{2}+k_{b e n} c_{0} \nabla^{2} d+k_{b e n} \zeta \frac{d}{\bar{d}} \nabla^{2} d+2 k_{b e n}\left(\nabla^{2} d\right)^{2}
$$

$c_{0}$ spontaneous curvature, $\zeta:=c_{0}-\bar{A} \partial_{c_{0}} A$, where $\bar{A}$ is the projected area: $\bar{A}=L^{2}$.

The interpretation of the former free energy is the following: the first term is the Helfrich bending associated with a tensionless surface with vanishing spontaneous curvature that the peristaltic fluctuations do not considerably influence. The second term corresponds to area stretching, the third and the forth reflect bending energetics due to peristaltic modes. Spontaneous curvature terms vanish in the undulations since both bilayers have opposite contributions.

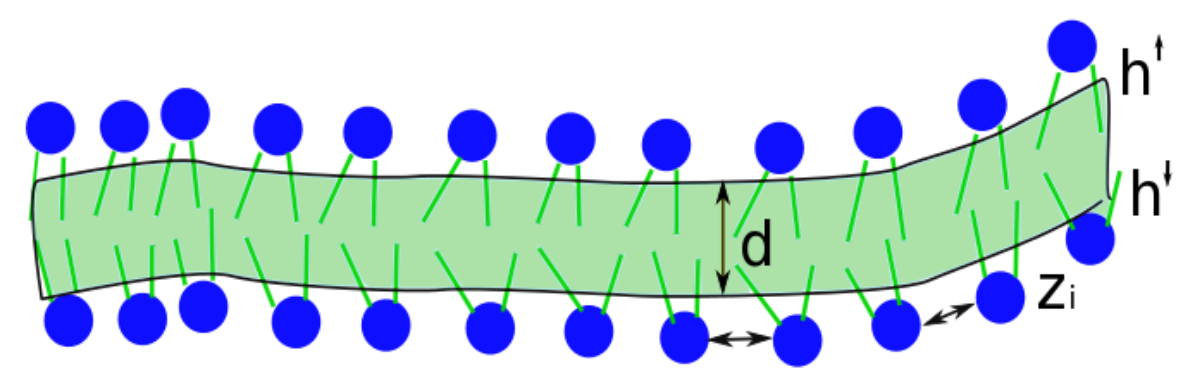

FiguRE 4.10: Sketch of the discrete description of a lipid membrane.

To take into account the microscopic protrusions we define the fields $z^{\uparrow}(x, y)$ and $z^{\downarrow}(x, y)$ that are defined by the conjunction point between the hydrophobic and the hydrophilic beads. The microscopic protrusions, $z^{ \pm}$, describe the local packing and reduces to $h^{ \pm}$in case of continuum description. They are important to describe the influence of the surface tension on the energy since it describes the exposition of the lipid tails to the solvent water:

$$
f_{\lambda}=\gamma_{\lambda}\left(\Delta A^{\uparrow}+\Delta A^{\downarrow}\right)+\frac{k_{\lambda}}{2}\left(z^{\uparrow^{2}}+z^{\downarrow^{2}}\right)
$$

In differential geometry the entire free energy is expressed as:

$$
\begin{aligned}
F & =\int \mathrm{d} x \mathrm{~d} y\left(k_{b e n} 2\left(\nabla^{2} h\right)^{2}+k_{\lambda}\left(z^{+^{2}}+z^{-2}\right)+\gamma_{\lambda}\left(\left(\nabla z^{+}\right)^{2}+\left(\nabla z^{-}\right)^{2}\right)\right. \\
& \left.+2 \gamma_{\lambda}\left(\nabla h \nabla z^{+}+2 \nabla d \nabla z^{-}\right)+\frac{k_{c o m}}{2} d^{2}+k_{b e n} c_{0} \nabla^{2} d+\frac{k_{b e n} \zeta d}{\bar{d}} \nabla^{2} d+\frac{k_{b e n}}{2}\left(\nabla^{2} d\right)^{2}\right)
\end{aligned}
$$

From this relation we can see that the height and the thickness fluctuations are decoupled.

In the work of [Branningan and Brown (2006, 2007)] the power spectrum of the former free energy is simplified in the following form where peristaltic and bending modes are separated:

$$
\begin{aligned}
\langle h(\boldsymbol{q}) h(-\boldsymbol{q})\rangle L^{2} & =\frac{1}{k_{b e n} q^{4}}+\frac{1}{2\left(k_{\lambda}+\gamma_{\lambda} q^{2}\right)} \\
\langle d(\boldsymbol{q}) d(-\boldsymbol{q})\rangle L^{2} & =\frac{1}{k_{b e n} q^{4}-4 \zeta k_{b e n} q^{2}+4 k_{e l} / \bar{d}^{2}}+\frac{1}{2\left(k_{\lambda}+\gamma_{\lambda} q^{2}\right)}
\end{aligned}
$$

The power spectrum includes the coupling between the fluctuations and the peristaltic motion considering the large compressibility of the system. The long wavelength region of the spectrum (small $q$, see fig 4.9 ) is fully represented in the equation (see eq 4.26 ). The correction by West and Brown (see eq 4.33 ) are used to accurately fit the spectrum on the short wavelength region (large $q \mathrm{~s}$, see fig 4.9 . After having fitted the spectra we realize that the contribution of $k_{\lambda}$ is negligible, $\left(k_{\lambda} \simeq k_{e l} / 1000\right)$ and conclude that (eq 4.27$)$ is accurate enough for determining $k_{e l}$. From the considerations of Branningan and Brown (2006)] (eq 4.26) is not a reliable approximation for wavelength on the order of the membrane thickness and in this regime diverges from (eq4.33). 
For a determination of the bending rigidity we fit the power spectrum using the equation: (eq 4.33). We have five parameters and two curves to fit (height and thickness power spectra) (see fig $4.9 \mathrm{p}$. We fit the first curve to obtain the bending rigidity of the membrane $k_{b e n}$ and the protrusion constants $k_{\lambda}, \gamma_{\lambda}$. Then we fit the second one and obtain the elastic coupling $k_{e l}$ and the area compressibility $k_{c o m}$.

The fit is performed by calculating the logarithm of the data and fitting the logarithm to assure the correct weighting of the maximum likelihood along the different orders of magnitude.

\section{7 tables}

In the following tables we summarize all the calculated values for the different model's parameters. We have investigated the change in the energetic contribution depending on the compressibility $k N_{b}$ (see table 4.3 ), on lipid architecture (see table 4.4 ) and on different lipid composition (see table 4.4 ).

compressibility change

TABLE 4.3: Summary of all the quantities calculated for the different values of the compressibility $k_{N} . D$ : diffusivity, $k_{\text {ben }}$ bending rigidity, $k_{e l}$ elastic coupling, $k_{\text {com }}$ area compressibility, $\bar{d}$ mean thickness, $\bar{a}$ mean area per lipid, $k_{\text {melt }}$ density compressibility.

\begin{tabular}{lccccl} 
& $k N_{b}=70$ & $k N_{b}=80$ & $k N_{b}=120$ & $k N_{b}=150$ & units \\
$D^{-1}$ & 76.6 & 124 & 3125 & 3636 & {$\left[t_{s} \Delta L^{2}\right]$} \\
$\bar{d}$ & 3.25 & 3.41 & 3.49 & 3.49 & {$[\Delta L]$} \\
$\bar{a}$ & 0.25 & 0.24 & 0.22 & 0.23 & {$\left[\Delta L^{2} / N_{c}\right]$} \\
& \multicolumn{7}{c}{ fluctuation } \\
$k_{\text {el }}$ & 38.75 & 50 & - & - & {$\left[k T / \Delta L^{2}\right]$} \\
$k_{\text {com }}$ & 22.72 & 31.5 & - & - & {$\left[k T / \Delta L^{2}\right]$} \\
$k_{\text {melt }}$ & 34.6 & 43.1 & - & - & {$\left[k T / \Delta L^{2}\right]$} \\
\multicolumn{7}{c}{} & \multicolumn{5}{c}{ spectrum } \\
$\gamma_{\lambda}$ & 10.68 & 12.42 & 11.49 & 11.83 & {$\left[k T / \Delta L^{2}\right]$} \\
$\zeta / \bar{d}$ & 0.14 & 0.098 & 0.072 & - & {$\left[\Delta L^{2}\right]$} \\
$4 k_{\text {el }} / \bar{d}^{2}$ & 7.85 & 8.9 & - & - & {$\left[k T / \Delta L^{4}\right]$} \\
$k_{\text {ben }}$ & 12.5 & 16.75 & 34.73 & 42.54 & {$[k T]$}
\end{tabular}

Between $k N_{b}=80$ and $k N_{b}=100$ the membrane has a phase transition between the fluid and the gel phase and we observe a consistent drop in the lipid diffusivity and increase in the bending rigidity. In the gel phase fluctuations are damped and the simulation time required to have a good sample of data to resolve peristaltic motion is several times the simulation time required in the fluid phase. It was hence not possible to gather enough statistics to calculate the elastic coupling in the gel phase.

We convert our energy using assuming that the system temperature is $300[K]$, hence $1\left[k_{B} T\right]=4.1418 \cdot 10^{-21}[\mathrm{~J}]$. Referring to values known in the literature for pure DOPC and POPC bilayers the bending modulus is $k_{b e n}=21.73\left[k_{B} T\right]$ [Kocun et al. (2011)] and conclude that our model is $30 \%$ softer than the real system.

\section{architecture change}

In the following we investigate the material properties of the membrane under change in the lipids architecture (see table 4.4).

The label 32 refers to long flexible chains (the polymer model), 8-10 refers to single tail lipids with 8 hydrophobic beads and 10 in total, the PC lipids have two tails, nine hydrophobic beads and four hydrophobic, the PE lipids have nine hydrophilic beads and three hydrophobic. We observe that the PC bilayers are stiffer than the PE. 


\begin{tabular}{lccccl} 
& 32 & $8-10$ & $\mathrm{PC}=9-13$ & $\mathrm{PE}=9-12$ & units \\
$D^{-1}$ & 0.67 & 76.6 & 48.8 & 39.6 & {$\left[t_{s} / \Delta L^{2}\right]$} \\
$\bar{d}$ & 4.69 & 3.25 & 2.26 & 3.05 & {$[\Delta L]$} \\
$\bar{a}$ & 0.41 & 0.25 & 0.32 & 0.24 & {$\left[\Delta L^{2}\right]$} \\
& & \multicolumn{3}{c}{ fluctuation } \\
$k_{\text {el }}$ & - & 50 & - & 32.6 & {$\left[k T / \Delta L^{2}\right]$} \\
$k_{\text {com }}$ & - & 31.5 & - & 20.8 & {$\left[k T / \Delta L^{2}\right]$} \\
$k_{\text {melt }}$ & - & 43.1 & - & 28.2 & {$\left[k T / \Delta L^{2}\right]$} \\
& & \multicolumn{5}{c}{ spectrum } \\
$\gamma_{\lambda}$ & - & 10.6 & 5.76 & 5.27 & {$\left[k T / \Delta L^{2}\right]$} \\
$\zeta / \bar{d}$ & - & 0.14 & -0.313 & 0.086 & {$\left[\Delta L^{2}\right]$} \\
$4 k_{\text {el }} / \bar{d}^{2}$ & - & 7.84 & 3.3 & 3.96 & {$\left[k T / \Delta L^{4}\right]$} \\
$k_{\text {ben }}$ & - & 12.5 & 9.54 & 8.9 & {$[k T]$}
\end{tabular}

TABLE 4.4: Summary of all the quantities calculated for the different lipid architectures $\left(k N_{b}=70\right)$.

\section{composition change}

The mechanical properties of the membrane can significantly change by membrane composition. In this section we show the change in the material properties by different lipid compositions.

\begin{tabular}{lcccl} 
& c-links [8-10] & PC+oil & PE+chol & units \\
$\bar{d}$ & 3.29 & 2.39 & 2.29 & {$[\Delta L]$} \\
$\bar{a}$ & 0.25 & 0.42 & 0.34 & {$\left[\Delta L^{2}\right]$} \\
& \multicolumn{5}{c}{ fluctuation } \\
$k_{\text {el }}$ & 39.7 & 18.5 & 14.1 & {$\left[k T / \Delta L^{2}\right]$} \\
$k_{\text {com }}$ & 27.0 & 13.4 & 9.3 & {$\left[k T / \Delta L^{2}\right]$} \\
$k_{\text {melt }}$ & 37.3 & 10.9 & 7.4 & {$\left[k T / \Delta L^{2}\right]$} \\
& \multicolumn{5}{c}{ spectrum } \\
$\gamma_{\lambda}$ & 15.5 & 12.4 & 6.8 & {$\left[k T / \Delta L^{2}\right]$} \\
$\zeta / \bar{d}$ & 0.56 & - & - & {$\left[\Delta L^{2}\right]$} \\
$4 k_{\text {el }} / \bar{d}^{2}$ & - & 6.26 & 4.72 & {$\left[k T / \Delta L^{4}\right]$} \\
$k_{\text {ben }}$ & 14.60 & 9.34 & 7.61 & {$[k T]$}
\end{tabular}

TABLE 4.5: Summary of all the quantities calculated for the different lipid composition $\left(k N_{b}=70\right)$.

For each bilayer we add $10 \%$ of the number of lipids of the the components written on the first line of (table 4.5) to the respective bilayer. The label c-links means the addition of a bond between two head groups represented by an harmonic spring with prefactor $k_{s p}=10\left[k_{B} T / \Delta L^{2}\right]$. The presence of cross links increases slightly the bending rigidity of the membrane. The bending rigidity of $\mathrm{PE}$ bilayers decreases with the presence of cholesterol and does not significantly change by adding oil in PC bilayers. The description we use to define lipids and other molecules is semplicistic and it is not able to capture the complexity of the atomic interactions. It was for example observed that the presence of cholesterol increases the bending rigidity of monosaturated lipid bilayers (DMPC, POPC) but does not influence the the bending rigidity of doubleunsaturated lipid bilayers (DOPC) Gracià et al. (2010)]. Looking at the architecture of the cholesterol in the model (see fig:4.1) we should probably define the molecule as a general fatty acid since we are not distinguishig between saturated and unsaturated bonds and we are not representing one of the most important feature of the cholesterol: the tetracyclic rings.

\section{8 modeling of an experiment}

One method to experimentally determine the stiffness of membranes consists of indenting them with the tip of an atomic force microscope (AFM) a lipid vesicle Li et al. (2011)] or a lipid [Kocun et al. (2011)] or polymeric [Kocun et al. (2010)] membrane. 
Figure 4.11: lhs) Sketch of the indentation of a vesicle by an elliptical tip. rhs) experimentally measured curves of the indentation of liposomes for different vesicle diameters, $d_{\text {ves }}$ Li et al. (2011)]. $r_{n}$ normal axis of the ellipsoid, $r_{l}$ lateral axis of the ellipsoid, $d_{i}$ distance between the ellipsoid surface and the bead $i$.
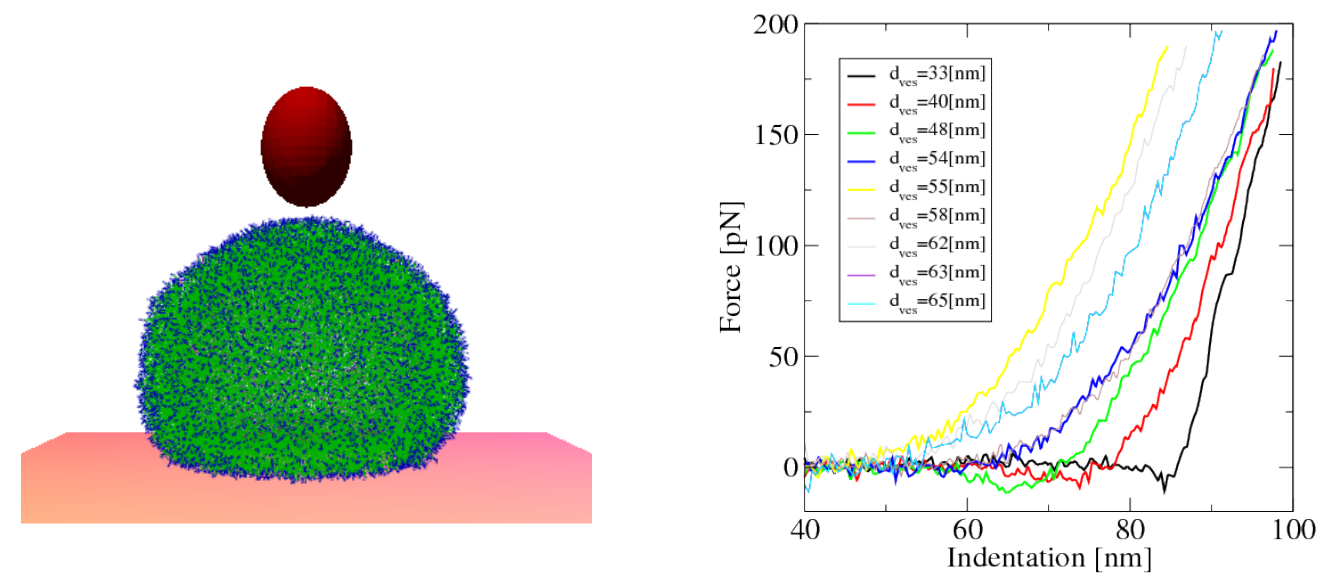

Since in such experiments the results depend both on the intrinsic membrane properties and the experimental boundary conditions, we run a sequence of simulations to control for the influence of the following properties that might be difficult to control in experiments:

- size of the tip

- speed of the tip

- contact angle between the supporting wall and the vesicle

- size of the vesicle

We perform the simulations using a vesicle sitting on a surface attractive towards the lipid head-groups by an attractive Lennard-Jones potential $\left(\epsilon_{\text {wall }}=1\left[k_{B} T\right]\right)$. We describe the tip as an ellipsoidal repulsive Lennard-Jones potential $\left(\epsilon_{t i p}=0.0004\left[k_{B} T\right]\right)$ defined by a normal $r_{n}$ and two equal lateral $r_{l}$ axis:

$$
\begin{aligned}
U_{t i p}\left(d_{i}\right) & :=\epsilon_{t i p}\left(\frac{r_{l}}{d_{i}}\right)^{12}-\epsilon_{t i p}\left(\frac{r_{l}}{d_{i}}\right)^{6} \\
d_{i}^{2} & :=\left(x_{n p}-x_{i}\right)^{2}+\left(y_{n p}-y_{i}\right)^{2}+\left(\frac{r_{l}}{r_{n}}\right)^{2}\left(z_{n p}-z_{i}\right)^{2}
\end{aligned}
$$

We slowly decrease the position of the tip, $z_{t i p}$, and calculate the response force $F_{z}$ which is the normal component of the total force exerted on the tip.

The stiffness, $k_{\text {stiff }}$ is given by the relation [Li et al. (2011)]:

$$
F_{z}=k_{\text {stiff }} \Delta z_{t i p}
$$

The interaction between the vesicle and the plane can be mapped to the experimental data via the ratio: vesicle height/vesicle diameter. We have mapped the force of the system comparing the line tension of the pore (see seventh chapter). The abscissa in the graph (see fig 4.12 refers to the distance between the center of the tip and the plane.

In the experimental set-up Li et al. (2011)] the tip size is $r_{l}=r_{n} \simeq 20[\mathrm{~nm}]$ and the tip speed is $3000[\mathrm{~nm} / \mathrm{s}]$. This tip speed is too slow to be able to run comparable simulations. We use a tip speed of $0.0625[\mathrm{~nm} / \mathrm{ns}]$ and we compare the results of the descending tip with a sequence of simulations where the tip remains at a fixed position. To perform the first calculation we average over five different indentation simulations with different starting conditions (fast motion). 


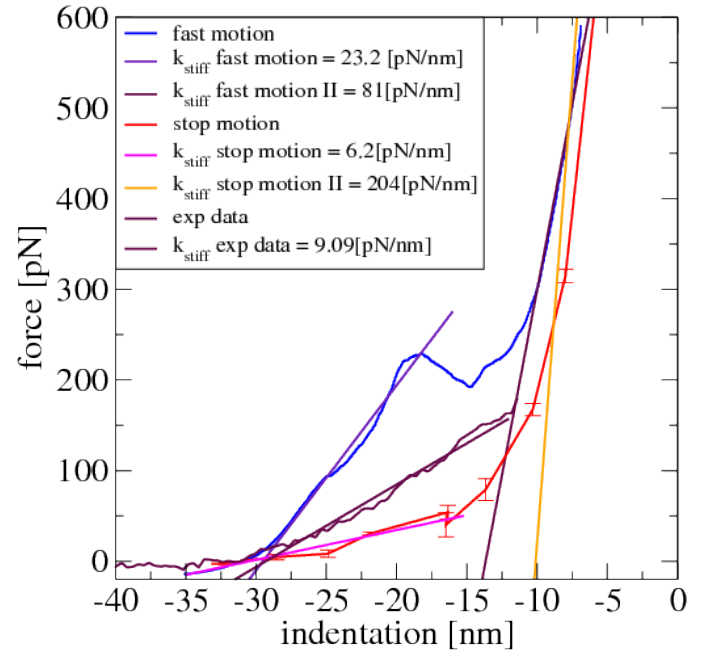

To perform the second calculation we have to equilibrate the system for each tip height for around $500\left[t_{s}\right]$ and then average the normal component of the force (stop motion). We use 12 different tip heights to reconstruct the indentation line. The ratio between the time of the two different calculations is estimated to be 10 times. The stop motion simulations are much more time demanding but the fast motion ones present an irregularity in the profile that is visible in the decrease of force with indentation (see fig 4.12). This fall is due to a residual momentum imposed on the vesicle in the transition where the membrane in contact with the tip is flat. The stop motion does not show thickness irregularity and provides a more realistic description of the real experiment as shown in (fig 4.11). The stiffness measured from the experimental indentation (see fig 4.11) has to be corrected by:

$$
\frac{1}{k_{\text {stiff }}}=\frac{1}{k_{\text {ind }}}-\frac{1}{k_{t i p}}
$$

The non equilibrium free-energy of the vesicle deformation can be calculated using the Jarzynski equality [Jarzynski (1997)]:

$$
\Delta F_{\text {ind }}=\ln \left\langle e^{-W}\right\rangle
$$

Where $W$ is the work done on the vesicle: $W=F_{z} \Delta z_{t i p}$.

Both in experiments and in simulations we identify two regions in the indentation curve: the first region corresponds to small indentations (until $35 \%$ of the total height), the second corresponds to large indentations (until twice the membrane thickness when the two bilayers start to fuse) (see fig 4.12). The ratio between the slope in the two regimes is around ten times both in experiments and simulations.

The value of the vesicle stiffness is increased by the effect of the fast motion of the tip as observed in experiment Li et al. (2011)]. The stiffness calculated by the stop motion indentation is $30 \%$ smaller than the experimental value and this is probably due to the softness of the model (a similar deviation is observed comparing the area compressibility measured in this chapter (see table 4.3 with the experimental data on synthetic lipid bilayers [Kocun et al. (2011)]).

\section{boundary condition contributions}

An interesting physical question is whether the stiffness is dependent on the size of the vesicle.
FiguRE 4.12: Indentation versus response for a $20[\mathrm{~nm}]$ radius liposome. The red curve refers to the average force between the tip and the liposome at fix tip height (stop motion), the blue curve refers to the fast speed motion. We have fitted both curves in the first, I, and second, II, indentation region (see text).

$k_{\text {ind }}$ slope of the indentation curve,

$k_{\text {tip }}=0.0390 \pm 0.005[\mathrm{nN} / \mathrm{nm}]$ stiffness of the tip of the AFM. 
Figure 4.13: lhs) Stiffness of the vesicle as a function of the curvature, the experimental data refer to the indentation of DMPC liposomes Li et al. [2011)], the simulation data refer to fast motion simulations. rhs) characteristics of the different indentation simulations. $\alpha_{\text {cont }}$ is contact angle between the vesicle and the substrate.

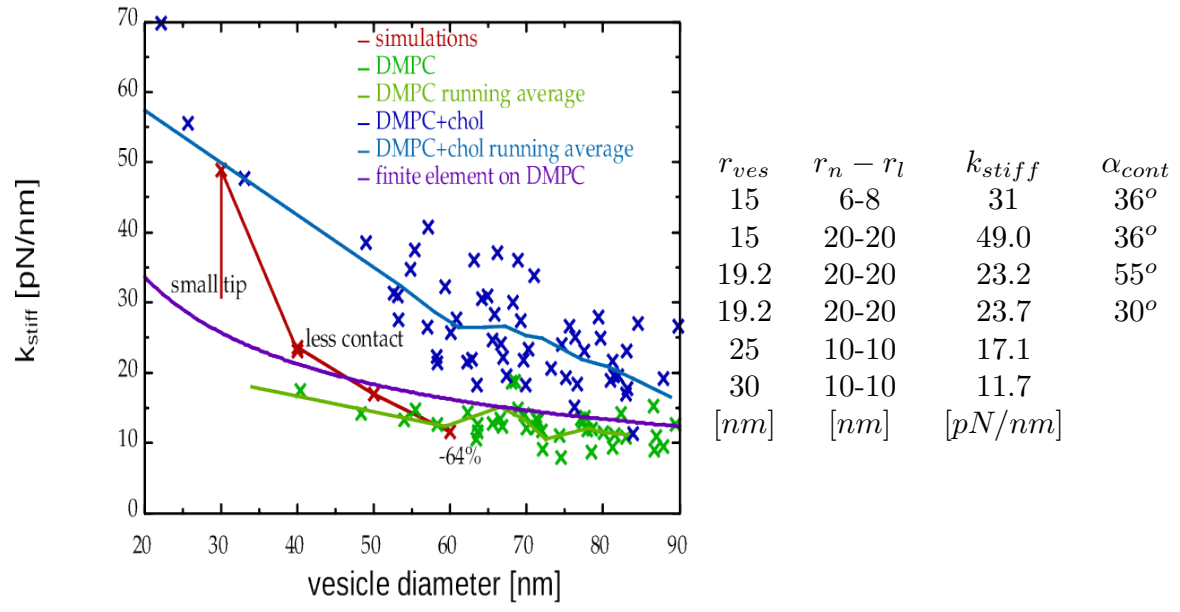

Lipid membranes are much more complex than elastic sheets, and at high curvature the compression of lipids, the thickness variation, and the induced curvature around the tip should influence the response force.

The influence of the tip size on the stiffness measurement is done by simulating the same indentation using different tip radii: $r_{n}=6[\mathrm{~nm}]$ and $r_{l}=8[\mathrm{~nm}]$ and $r_{n}=r_{l}=$ $20[\mathrm{~nm}]$.

From the table (see fig 4.13 we observe a change of $30 \%$ in the stiffness.

To analyze the influence of the speed we have compared a single indentation simulation (tip speed $=0.0625[\mathrm{~nm} / \mathrm{ns}]$ ) with a sequence of simulations where the tip was at a fixed constant height. In the graph 4.12 we can observe the difference in the indentation curve depending on the speed of the tip.

We observe that the contact angle between the vesicle and the substrate $\alpha_{\text {cont }}$ does not influence significantly the indentation curve (see table 4.13 ).

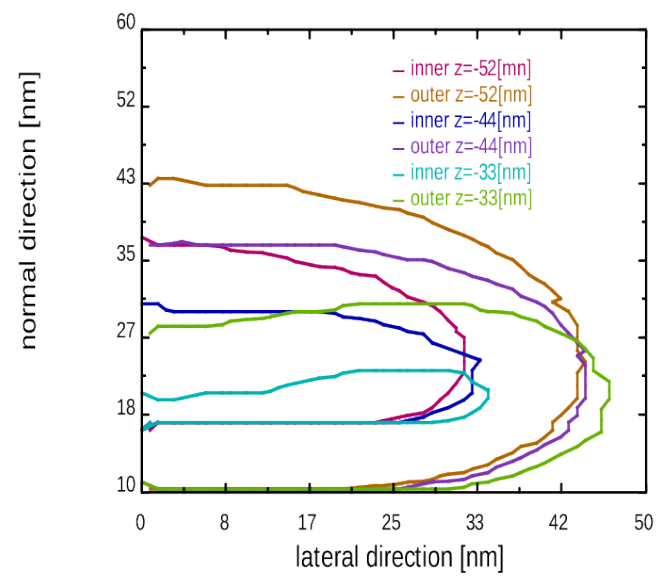

A significant change in the stiffness is caused by the radius of the vesicle. We can see that the resistance of the vesicle to indentation decreases of $60 \%$ from doubling the vesicle radius from 15 to $30[\mathrm{~nm}]$. With respect to the experiments we calculate smaller values of the vesicle stiffness but we observe a similar behaviour in the dependence between the vesicle radius and the stiffness.

With respect to the thin shell finite element calculation $\mathrm{Li}$ et al. (2011)] we can extract more precise information about the shape deformation of the vesicles. We calculate the density plot of an indented vesicle on a slab passing through the center 
of the vesicle and with a width of $4[\mathrm{~nm}]$.

We have decided not to calculate the radial density profile because the fluctuation in shape distorts the thickness profile at large radial distances. From density profiles we extract the isolines that give precise information about the local curvature and thinning of the membrane (see fig 4.14), both information are not available neither in experiments nor in finite elements calculations.

This small set of simulations shows clearly that the deformation rate, the size of the indenting ellipsoid and the radius of the vesicle all have a huge effect on the simulated vesicle stiffness. A more detailed analysis of the parameter space will be necessary to map the exact relations and to check if the continuum mechanics approach followed by Li et al. (2011)] ignores important physical aspects of the experiments.

\section{9 conclusion}

In this chapter we have identified the lipid architecture and the parameter range where the lipids self-assemble in fluid planar bilayer. The input parameters of the model can be mapped into the macroscopic measurable values calculating the mechanical properties of the planar membrane. We calculate at first the thickness profile of the membrane and the diffusivity of lipids to set the length and time scale of the system. Successively, we have shown some relevant energetic contributions in shape deformation of bilayers. These values will be used in the next chapters as reference to study the local modification around the fusion objects and to map the continuous model calculations. As a novel application we have used our solvent-free coarse-grained model to calculate the stiffness of liposomes and compare the results with finite elements calculations and experiments. We have shown the dependence of the final measurements on the indetermination of the environmental conditions and achieved a good qualitative agreement with the experiments. 
CHAPTER 4. MECHANICAL AND PHASE PROPERTIES OF PLANAR conclusion MEMBRANES 


\section{Chapter 5}

\section{stalk dynamics and morphology}

The stalk is a lipid bridge between two lipid membranes and a fundamental intermediate structure in the process of membrane fusion. The formation and evolution of the stalk is a collective phenomenon, which involves the interaction and change of conformation of many lipids.

We use our coarse-grained solvent-free model to simulate a stalk between two opposed membranes. The absence of solvent molecules avoids the non trivial problem of re-equilibrating the number of solvent molecules between the two bilayers present in explicit solvent models and the hydration repulsion can be controlled via the interactions between the lipid head groups. Depending on the type and architecture of lipids we can change the stability and morphology of the stalk. Circular stalks are metastable and we calculate the average density profile and fluctuations of their radius. Small hydrophobic chains (oil) are added in the hydrophobic layer of the membrane preferentially go to the lower and upper ends of the stalk, where the membrane is slightly indented and the hydrophobic tails stretch to uniformly fill the space, and relax the total tension. Linear stalks formed by more asymmetric lipids are stable and span the simulation box over the periodic boundary conditions and we calculate their stiffness. We compare the thickness profile and the bilayer repulsion with different models and experimental data. To compare the solvent-free model with the explicit solvent models and experiments we change the head group interactions to mimic the hydration repulsion between the membranes. We estimate the bilayer repulsion depending on the hydration and lateral tension.

\section{1 introduction}

The adhesion and fusion between bilayer membranes gives rise to the mixing of lipids between the neighboring leaflets Markvoort and Marrink (2011); Stiasny and Heinz (2004); Kozlovsky et al. (2004)]. This process involves the building of a lipid bridge, called stalk, where the lipids of the two opposed leaflets mix with each other.

The shape, the free-energy cost and stability and the mechanical properties of the stalk strongly depend on the lipid composition. We identify two lipid architectures that correspond to the inverted hexagonal or lamellar phase in the self-assembled aggregates (see fig 4.2 ). The second architecture has just one bead more in the head-group, this suggest us to call the first lipid type $\mathrm{PE}$ and the second $\mathrm{PC}$ recalling the different headgroups of the lipid membranes that correspond to the same self-assembled phases.

To create a stalk we prepare two opposed bilayers and induce a stalk using a cylindrical 
$U_{\text {ext }}$ external harmonic potential, harmonic potential:

$\left(x_{e x t}, y_{e x t}\right)$ is the position of external potential, $\epsilon$ is the energy prefactor.

$$
U_{\text {ext }}=\sum_{i}^{N} \epsilon\left(\left(x_{i}-x_{e x t}\right)^{2}+\left(y_{i}-y_{\text {ext }}\right)^{2}\right)
$$

In case of PE lipid membranes the stalk, after eliminating the external potential, elongates linearly until the two opposed caps fuse together and the stalk expands along the whole box length (see fig 5.3. In case of a PC lipid membrane the stalk preserves the circular shape induced by the external field (see fig 5.4.

We develop two methods to describe the two stalk morphologies making use of the particular symmetry of the two stalks. In the case of PE membranes we study shape fluctuations reconstructing the linear shape of the stalk. In the case of PC membranes we construct the torus that at best envelop the hour glass shape of the stalk. The position of the center of mass of the stalk and shape fluctuations give us important information about the diffusion and the stiffness of the stalk.

We calculate the thickness profile in different models and compare the characteristic sizes of the stalk with the experiment. We associate profiles with similar hydration level that we quantify by the width of the water layer. In a solvent-free model the hydration level is represented by rescaling the virial coefficient of the head-group interactions, $v_{B B}$. This virial coefficient changes effectively the interaction with the implicit water molecules and hence the hydration between the two bilayers. We show how the hydration layer works as parameter for the transition between the lamellar to the inverted hexagonal phase in experiments, explicit and implicit solvent models.

We quantify the energy change connected with the variation of $v_{B B}$ studying the bilayer repulsion between the two leaflets Leikin et al. (1993); Rand and Parsegian (1989); Kozlov et al. (1994); McIntosh and S.A.Simon (1994); Rand et al. (1988)].We use the same method to calculate the bilayer repulsion between membrane with different lateral tensions.

Between two opposed membranes stalk and pore formation are two competing processes that depend on the lateral tension and hydration. We observe that in the case of stalk formation preceding the pore formation the two bilayers fuse.

\subsection{Martini simulations}

The Martini simulations were performed with the GROMACS software package, version 3.3.2 van der Spoel et al. (2005)]. The systems considered in this work were studied under periodic boundary conditions using the Martini coarse-grained model Marrink et al. [2007)]. The coarse-graining procedure lumps four water molecules into one coarse-grained bead and the atomistic lipid into the coarse-grained representation of the picture (see fig: 5.1). Covalent bonds of lipids are modeled by springs, and the stiffness of the lipid tails is provided by angle potentials.

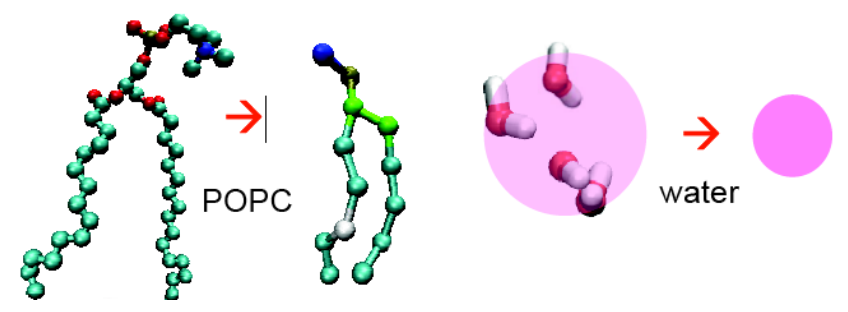

The polarity of the groups is modeled by effective Lennard-Jones parameters. LennardJones interactions are truncated at $1.2[\mathrm{~nm}]$. The zwitterionic character of the lipid molecules is modeled by charges on the choline and phosphate groups. A $1.2[\mathrm{~nm}]$ cut-off was used for the neighbor list, which is updated every 10 time steps. The
Figure 5.1: Coarse-grained representation of the Martini force field for POPC lipids and water molecules. Color code: red is oxygen, cyan is carbon, white is hydrogen in water and double bond in coarse-grained, green is glycerol backbone, blue is nitrogen, and tan is phosphorus. 
effective time step used was $160[f s]$ (the effective time scale is defined via diffusion of lipid molecules Marrink et al. (2007)]). Simulations were conducted at $300[K]$ in the $N P_{\perp} P_{\|} T$ ensemble by coupling the lipids and the water separately to a heat bath using a Berendsen thermostat Berendsen et al. (1984)] with a relaxation time of $0.4[p s]$. The normal and lateral dimensions to the bilayer were scaled independently to maintain a pressure of $1[b a r]$ in each direction, corresponding to zero tension using a Berendsen barostat Berendsen et al. (1984)] with a relaxation time of $0.8[p s]$.

The Martini simulations described in this chapter were performed by my colleague Yuliya Smirnova.

\section{3 simulation details}

In the following chapter three different models are used.

DFT: The first model is the solvent-free coarse-grained model introduced in the first two chapters of this thesis that we call in short DFT (from density functional theory).

The soft solvent-free simulations are run in the $N P_{t} T$ ensemble, the system contains 4300 chains divided in two bilayers separated by a short distance. At the initial stage of the simulation an external cylindrical field induce locally the lipid mixing between the membranes. The equilibrating transient is excluded from the further analysis.
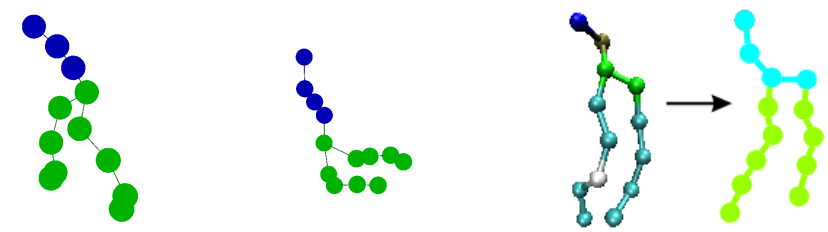

Martini: The second model is the Martini 2.0 force field Marrink et al. (2007)]. The simulations were performed in NPT ensemble with $T=300[K]$ and $P=1[b a r]$. All systems are composed by two POPC bilayers, each containing 512 lipids. Each small system was simulated for $1.2[\mu] \mathrm{s}$ and each large system for $2.4[\mu] \mathrm{s}$.

Polymeric: The third model uses an explicit solvent and describes long and flexible chains (like polymers) Müller et al. (2003)]. Each bilayer contains about 8500 chains, the polymers are described by 32 beads, 10 of them are hydrophilic. The unit length is expressed in end-to-end distance of the chains and the system size is: $(6,6,8)\left[R_{e}\right]$.

For each analysis the system is equilibrated simulated for some period of time to obtain at least 500 snapshots.

\section{4 building a stalk}

We use two different lipid architectures to reproduce the lamellar (PC head groups of the lipids) and the inverted hexagonal phase (PE head groups of the lipids).
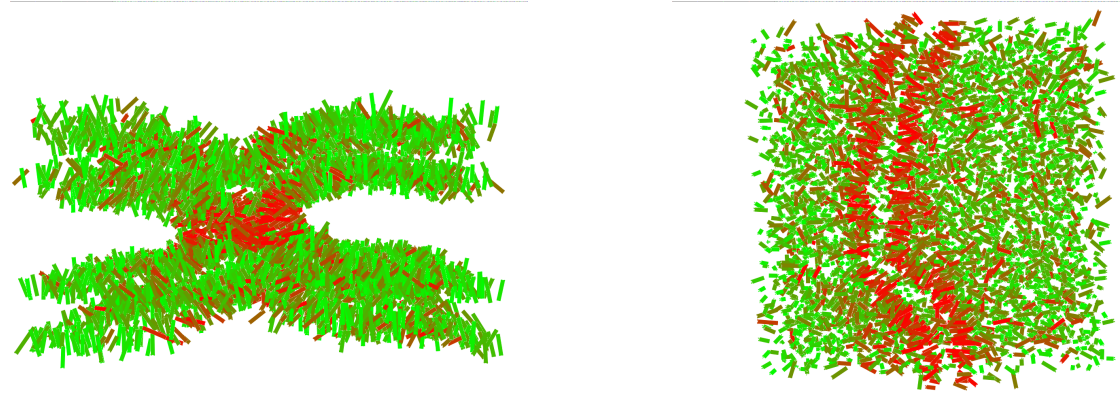

We induce the lipid mixing using an harmonic cylindrical potential to collect lipids in a small region between the two membranes. After a short equilibration time the stalk
Figure 5.2: Representation of a PE (lhs) and a PC (middle) lipid type for the DFT model. Parametrisation of the Martini force field into diblock lipids (rhs). The phosphate group is assigned to the hydrophilic block of the lipid. 
FiguRE 5.4: lhs) side view of a circular stalk, rhs) top view of a circular stalk. In red are drawn the directive of the chains between $60^{\circ}$ and $120^{\circ}$ degrees, in green the others. acquires a linear (see fig 5.3) or circular (see fig:5.4 shape.

To describe the static and dynamic properties of the stalk we make use of the particular symmetry to reduce the dimensionality of the problem. We develop a method to describe the line along the linear stalk and a method to describe the torus that fits at best the hour-glass shape of the circular stalk (see next section).
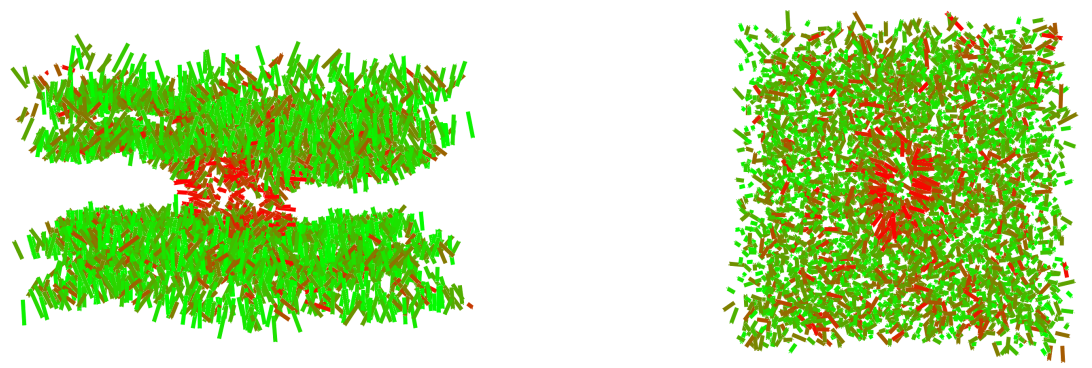

\section{5 shape reconstruction of a linear stalk}

To calculate the shape of the linear stalk we perform the following steps.

We consider only the chains of the opposed leaflets. The conjunction point between the chains is used to define the upper and lower surface by sampling the membrane area on a large grid and by calculating the center of mass for each square. The surfaces are successively smoothed by B-splines (see fig 5.5 .
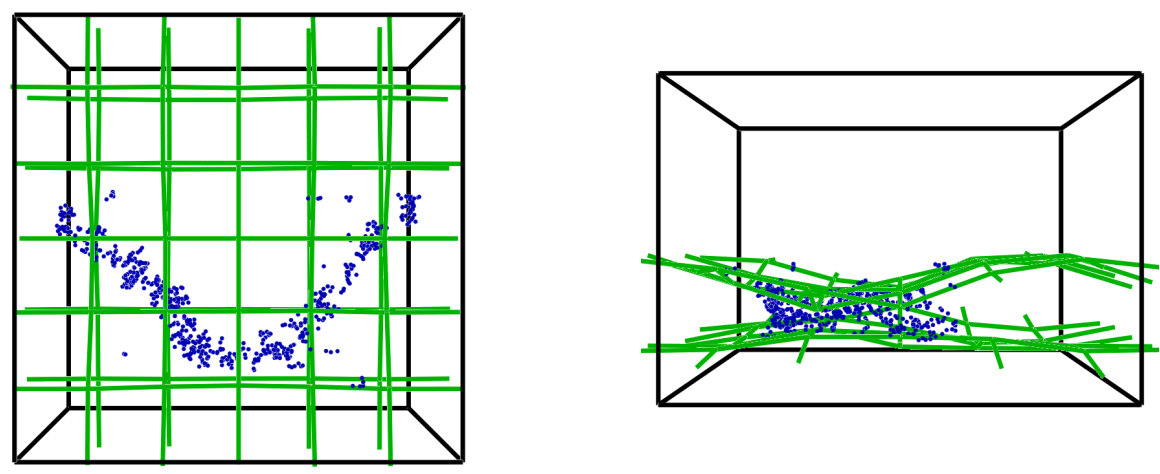

The points between the two opposite midplanes (blue points in fig 5.5 are projected on the xy-plane. To define the line that describes at best the large band in the middle of the plot (see fig:5.7) we remove the influence of the points that are far away from the center of the line.

The procedure is the following:

- The first approximation line is calculated binning the space in the $\mathrm{x}$ direction with $N_{b i n}$ number of bins.

- The chains composing the stalk are mainly perpendicular to the lipids sitting in the bilayer. To enhance their contribution in the average we calculate the average center of mass weighting the point with the following weight $w_{i}=1-\left(\frac{\alpha_{i}-\pi / 2}{2}\right)^{2}$ where $\alpha_{i}$ is the angle of the corresponding chain with the normal.

- We smooth the calculated line interpolating with B-splines do induce correlation between neighboring bins. 


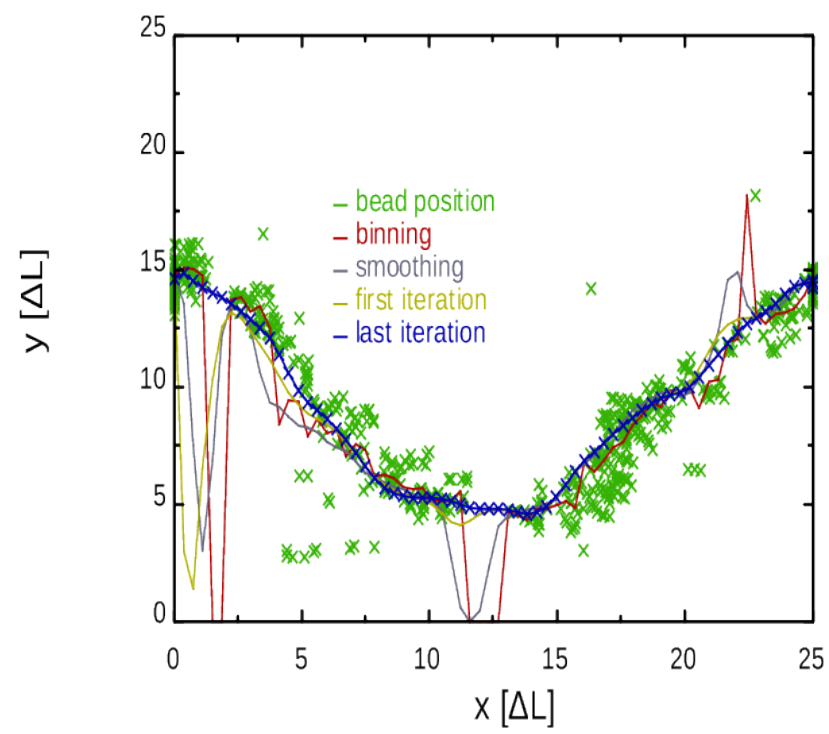

- To reduce the high frequency fluctuations we recalculate the average position of the bin by weighting with respect to the former line position. $w_{i}=1-\left(\frac{y_{i}-y_{i}^{-1}}{L_{y} / 2}\right)^{2}$ where $y_{i}$ is the particle position and $y_{i}^{-1}$ is the former position correspondent to the same bin $i$.

- We smooth again.

We apply this procedure six times to remove long jumps between two consecutive points.

\section{6 position and size of a circular stalk}

The radial profiles are extremely sensitive to the determination of the center considering the small size of the stalk radius. The stalk is composed by a handful of lipids, about $1 \%$ of the total, and diffuses over the box size. To identify the position of the center and to follow the diffusion of the stalk we had therefore to develop an ad hoc method.
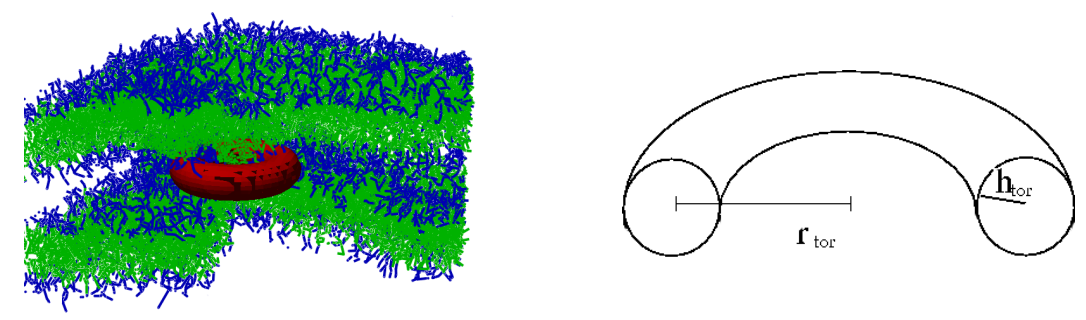

To trace the position we have build a torus (see fig 5.7) described by the following squared distance function $d^{2}\left(\boldsymbol{x}_{i}\right)$ :

$$
\begin{array}{r}
d^{2}\left(\mathbf{x}_{i}\right)=\left|\left(r_{\text {tor }}-\Delta r_{i}\right)^{2}-h_{\text {tor }}^{2}+\left(z_{i}-z_{\text {tor }}\right)^{2}\right| \\
\Delta r_{i}:=\sqrt{\left(x_{i}-x_{\text {tor }}\right)^{2}+\left(y_{i}-y_{\text {tor }}\right)^{2}}
\end{array}
$$

The torus is repulsive to the hydrophobic beads, attractive to the hydrophilic, has a
energy gain for small lateral radius $h_{\text {tor }}$ and an energy loss for small normal radius $r_{\text {tor }}$.
Figure 5.6: The green points represent the beads between the opposed midplanes. The lines represent the sequence of iterations to reconstruct the final shape of the linear stalk.
FIGURE 5.7: lhs) Sketch of a torus embedding a stalk. rhs) Definition of the characteristics of a torus, the normal and lateral radii: $r_{t o r}, h_{t o r}$. 

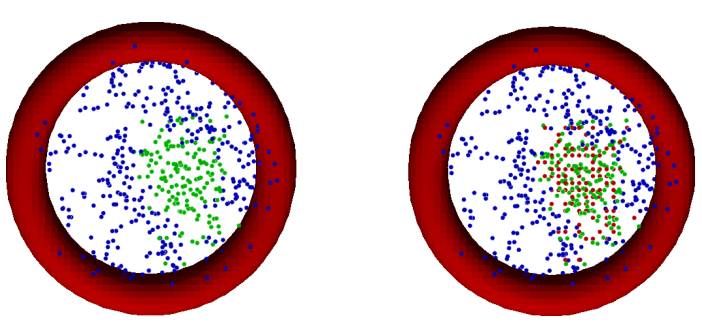

FiguRE 5.8: I) Projection on a plane of the hydrophilic (blue) and hydrophobic (green) beads inside the torus. II) We count how many grid sites are occupied by hydrophobic beads (number of red dots). III) we update the position and the radius of the stalk calculating the center of mass and the area occupied by the red dots.

\section{$\epsilon_{\alpha}(i):=\left\{\begin{array}{ccc}\epsilon_{A} & \text { if } & \operatorname{type}(i)=A \\ \epsilon_{B} & \text { if } & \operatorname{type}(i)=B\end{array}\right.$}

$\alpha=\{A, B\}$

$$
\text { by }
$$
$\left.\epsilon_{\text {tor }}, y_{\text {tor }}, z_{\text {tor }}\right)$. We have chosen as parameters: $\epsilon_{A}=8000, \epsilon_{B}=-50, \epsilon_{\text {lat }}=700$, stalk/torus interaction and we use a Metropolis like acceptance criterion to accept or decline the move:

$$
\operatorname{acc}\left(m \rightarrow m^{\prime}\right)=\min \left(1, e^{\left(E_{t o r}^{\text {old }}-E_{\text {tor }}^{\text {new }}\right)}\right)
$$

After tens of iterations the new position and size of the torus is set. To refine the definition of the torus we count how many beads are contained inside the torus. We sample the area inside the torus using a lattice of $36 \times 36$ sites and count how many of the sites are occupied by hydrophobic beads. We calculate the area, $A_{t o r}$, by the fraction of occupied sites (red dots in picture 5.8 ) and use it to define the new radius of the torus $r_{t o r}=\sqrt{A_{t o r} / \pi}$ and calculate the center of mass of the beads in that area to update the position of the torus. The last size and position of the torus is used as starting configuration for the next snapshot. Averaging over all configurations we measure an average number of 27 lipids involved in the definition of the stalk size.

From the evolution of the position of the stalk we calculate the mean square displacement (see eq 4.2 and measure the diffusivity of the stalk about one third of the lipid diffusivity $D_{\text {stalk }} \simeq D_{\text {lipid }} / 3$.

\section{7 line tension of the stalk}

In this section we calculate the line tension of a linear stalk.

$\lambda$ line tension and $k_{\text {ben }}$ bending rigidity of the linear stalk.

The Hamiltonian of the shape deformation for a linear stalk is:

$H=\frac{1}{2} \int_{0}^{L_{x}} \mathrm{~d} x\left(\lambda(\nabla y(x))^{2}+k_{b e n}\left(\nabla^{2} y(x)\right)^{2}\right)-L_{x} \lambda \quad F=-\ln \int D[y(x)] \mathrm{d} x e^{-H(y(x))}$

The thermodynamic tension, $\Lambda$, is the energy of the stalk per unit length:

$$
\Lambda=\frac{F}{L_{x}}=-\frac{1}{L_{x}} \ln \int D[y(x)] e^{-H(y(x))}
$$

Similarly to the calculation performed in (see eq 4.26) we obtain the following power $q_{x}(n):=2 \pi n /\left(N_{b i n} L_{x}\right), N_{b i n}$ spectrum:

$$
\left\langle\hat{y}\left(q_{x}\right)^{2}\right\rangle L_{x}^{2}=\frac{1}{k_{b e n} q_{x}^{4}+\lambda q_{x}^{2}}
$$

that we use to fit the data and extract the stiffness, $\lambda$, and the bending rigidity, $k_{b e n}$, of the linear stalk (see fig 5.7). The presence of the cholesterol reduces the bending rigidity of the stalk as the bending rigidity of the membrane as already calculated in the previous chapter (see table 4.5. 


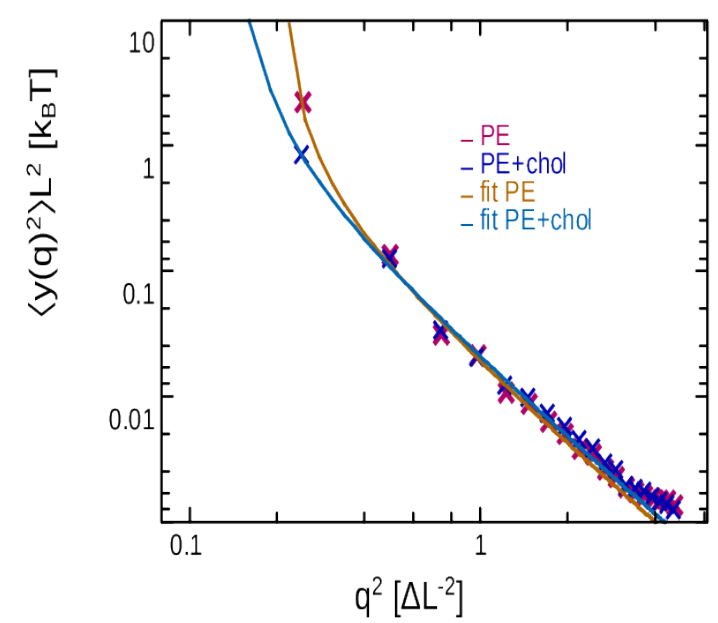

\begin{tabular}{ccc|ccc} 
& $k_{\text {ben }}$ & $\lambda$ & & $k_{\text {ben }}$ & $\lambda$ \\
\hline $\mathrm{PE}$ & 64.5 & -14.0 & $\mathrm{PE}+\mathrm{chol}$ & 56.0 & -8.8 \\
\hline & {$\left[k_{B} T \Delta L\right]$} & {$\left[k_{B} T / \Delta L\right]$} & & {$\left[k_{B} T \Delta L\right]$} & {$\left[k_{B} T / \Delta L\right]$}
\end{tabular}

\section{8 rigidity of the stalk}

In case of a circular stalk we measure the radius fluctuations of the embedding torus.
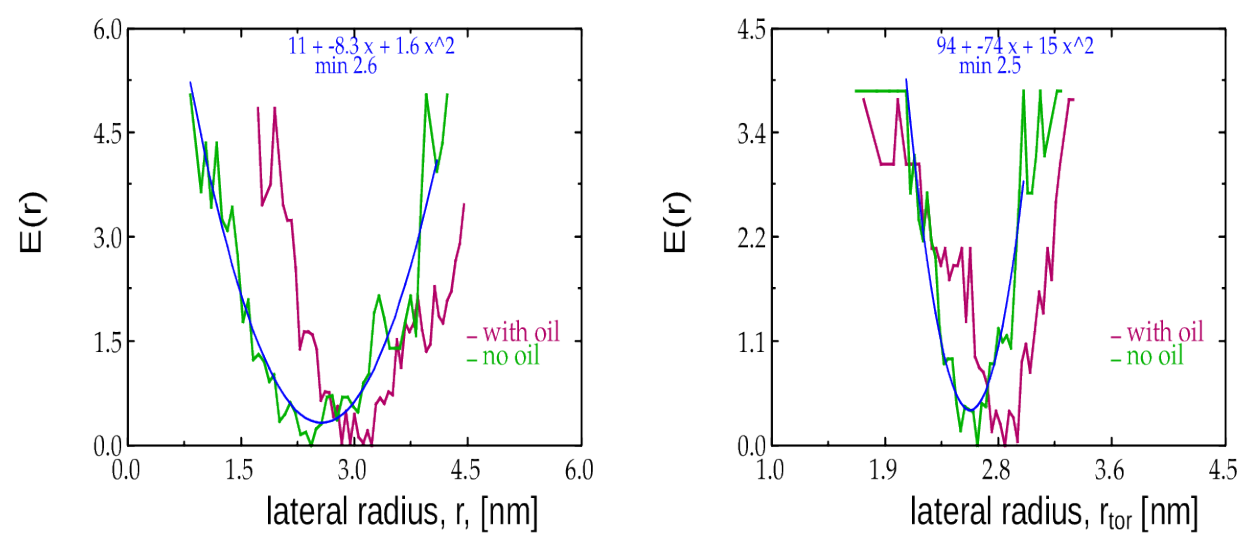

Due to the softness of our model we calculate the probability distribution of the stalk radius and define the rigidity of a linear stalk using the equation:

$$
P(r)=\int \mathrm{d} r^{N} e^{-H\left(r^{N}\right)} \delta\left(r_{\text {tor }}-r\right) \quad F=-\ln P(r)=: \frac{1}{2} k_{r i g}\left(r-r_{e q}\right)^{2}
$$

where we have used the harmonic approximation to fit the data (see fig 5.10). We

\begin{tabular}{cccccc} 
& DFT & DFT+oil & Martini & Martini+oil & \\
\hline$k_{\text {rig }}$ & 1.6 & 2.3 & 13 & 15 & {$\left[k_{B} T / \mathrm{nm}^{2}\right]$} \\
$r_{\text {min }}$ & 2.61 & 3.21 & 2.53 & 2.77 & {$[\mathrm{~nm}]$}
\end{tabular}

observe that the presence of the oil expands the radius of the stalk and increases its rigidity. The detailed discussion on the oil partitioning inside the stalk is developed in the seventh chapter of this thesis.
FiguRE 5.9: The power spectrum of the line fluctuations and the linear fits.

TABLE 5.1: Values of the stiffens, $k_{s t i f f}$, of the circular stalk for DFT and Martini with and without the presence of oil.

FiguRe 5.10: Parabolic approximation of the stiffens of the stalk from the fluctuation of its radius for the soft solvent-free (lhs) and the Martini models (rhs).

TABLE 5.2: Values of the rigidity, $k_{\text {rig }}$, of the circular stalk for DFT and Martini with and without the presence of oil. 


\section{9 density profile}

The determination of the center of the stalk allows us to calculate the radial density profiles.

Figure 5.11: Thickness profile of a stalk: lhs) in green the density of the hydrophobic beads, in blue the density of the hydrophilic. rhs) the characteristic sizes of the stalk are the trunk, $d_{t}$, the indentation, $d_{i}$, the hydration layer, $d_{w}$.

Figure 5.12: Radial density profiles obtained from I) experiment Aeffner et al. (2009), II) Martini, III) polymeric, and IV) DFT simulations.
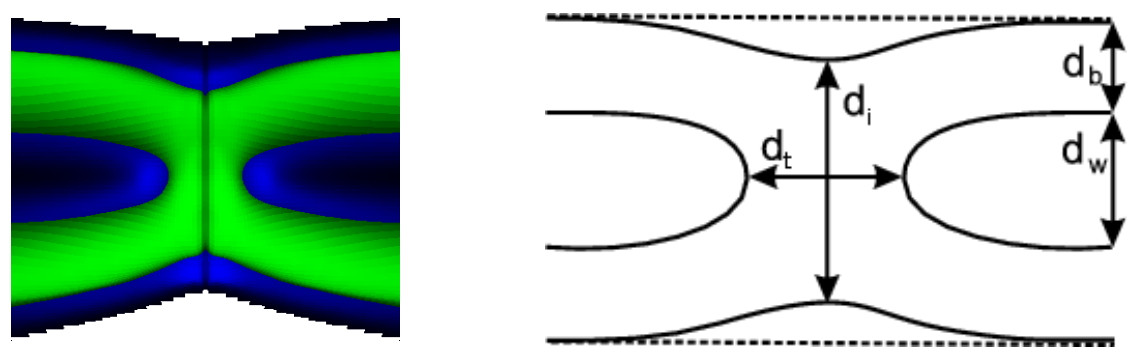

The structure of the stalk is schematized in the cartoon of the figure (5.11). From the density profile we can extract the characteristic sizes of the stalk that depend on the membrane composition or on the hydration. The characteristic sizes are the trunk, $d_{t}$, the indentation, $d_{i}$, and the hydration layer thickness, $d_{w}$ Aeffner et al. (2009)]. We compare stalk structures obtained from different simulation models and the experiment Aeffner et al. (2009)].

For each model we have used the method described above to calculate the center of mass of the stalk, which is needed for the calculation of the density profiles. In the experiment the stalk structure obtained from the electron density profile, the maximum of the density corresponds to the phosphate group of a lipid molecule. The characteristic sizes are calculated measuring the distances between isolines corresponding to half maximum of the hydrophobic density.

All lengths are rescaled by the membrane thickness for the comparison as defined in the previous chapter (see fig 4.4.
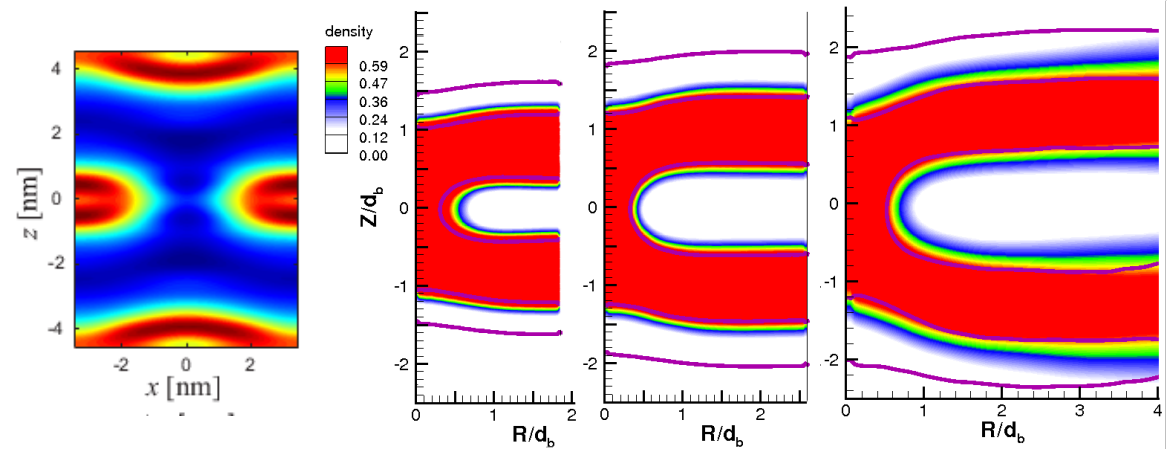

To point out the universality of these structures we compare in the table the characteristic ratios of the stalk for different hydrations (see fig 5.3 .

The definition of hydration depends on the model and on the experimental set-ups. In the experimental case the hydration is controlled by the vapor pressure ( $\mathrm{RH}$ : relative humidity) of the surrounding solvent Aeffner et al. (2009)], for explicit solvent models it is controlled by the water layer $d_{w}$, and for implicit solvent models by the head group interactions. To compare stalk structures from different models and experiments we refer to the thickness of water layer, $d_{w}$, as the positions of the half maximum of the hydrophobic density (see fig 4.4 ) and compare the different structure by this quantity. From the table (see table 5.3 we can draw the following conclusions. The hydration level has a large effect on the stalk indentation, $d_{i}$, and in the ratio $d_{i} / d_{t}$, both in Martini simulations and experiments. In Martini simulations the stalk is at $5 N_{w} / N_{l}, d_{w}=2.04[n m]$ not circular and largely elongates. At $8 N_{w} / N_{l}, d_{w}=4.16[n m]$ 


\begin{tabular}{|c|c|c|c|c|}
\hline$d_{w}$ & & $d_{i} / d_{b}$ & $d_{t} / d_{b}$ & $d_{w} / d_{b}$ \\
\hline 2.04 & Martini @ $5 N_{w} / N_{l}$ & 2.1 & 0.95 & 0.67 \\
\hline 2.11 & Martini @6 $N_{w} / N_{l}$ & 2.2 & 0.78 & 0.70 \\
\hline 2.24 & Martini $@ 7 N_{w} / N_{l}$ & 2.3 & 0.74 & 0.73 \\
\hline 3.33 & $\exp @ R H=34 \%(\mathrm{DPhPC})$ & 2.3 & 0.9 & 0.68 \\
\hline 3.88 & $\exp @ R H=78 \%(\mathrm{DOPC})$ & 2.1 & 0.91 & 0.73 \\
\hline 2.12 & solvent-free $@ v_{B B}=-1.0$ & 2.13 & 1.13 & 0.60 \\
\hline 2.75 & solvent-free @ $v_{B B}=-0.5$ & 2.33 & 1.08 & 0.78 \\
\hline 4.08 & solvent-free @ $v_{B B}=-0.1$ & 2.16 & 0.97 & 1.16 \\
\hline \multirow[t]{2}{*}{4.29} & solvent-free $@ v_{B B}=-0.1+$ oil & 2.08 & 1.14 & 1.22 \\
\hline & Polymeric & 2.6 & 0.8 & 1.1 \\
\hline
\end{tabular}

TABle 5.3: Characteristic ratios of the stalk in different models and experimental conditions. $N_{w} / N_{l}$ is the number of water molecules between the two bilayers divided by the total number of lipids, $R H$ is the relative humidity.

the stalk is extremely thin, which indicates that the stalk structure is in $4[\mu s]$ simulations highly metastable for this hydration. At $9 N_{w} / N_{l}$ the stalk disappears after $3.5[\mu s]$ via splayed lipid bond configuration.

Changing the level of hydration we are moving the membrane from the inverted hexagonal phase to the planar phase passing through the rhombohedral phase. The same happens in the experiments and in the solvent-free simulations where changing the parameter $v_{B B}$ from -1 to -0.5 we observe a transition from the inverted hexagonal to the rhombohedral phase.

The identification of the exact hydration level for the phase transition is not a trivial problem. Stable stalks correspond to the rhombohedral phase and their formation corresponds to a free-energy gain with respect to the system of two isolated bilayers. Similar calculations to determine the free-energy gain/loss in the formation of the stalk were already performed in our group using the string method Müller et al. (2012)] or the lipid chemical potential difference Norizoe et al.(2010)]. These simulations suggest the regime for the parameter $v_{B B}$ for the inverted hexagonal/rombohedral/planar phase transition.

\subsection{0 stalk elongation}

The decrease in hydration and the presence of oil have as effective result the elongation of the stalk. To quantify the elongation of the stalk we calculate the acylindricity which is defined as the relative difference between the first two principal moments of the gyration tensor. The gyration tensor is defined as Blavatska and Janke (2010); Rudnick and Gaspari (1986); Theodorou and Suter (1985)]:

$d_{1}, d_{2}=\{x, y, z\}$

$$
S_{d_{1} d_{2}}=\frac{1}{3 N_{\text {tor }}^{2}} \sum_{i}^{N_{\text {tor }}} \sum_{j}^{N_{\text {tor }}}\left(x_{i}^{d_{1}}-x_{j}^{d_{1}}\right)\left(x_{i}^{d_{2}}-x_{j}^{d_{2}}\right)
$$

Where $N_{t o r}$ is the number of particles included in the torus and $o\left(\Delta r, r_{t o r}\right)$ the occupation function:

$$
N_{\text {tor }}=\sum_{i=1}^{N} o\left(\Delta r_{i}, r_{\text {tor }}\right) o\left(\Delta z_{i}, h_{\text {tor }}\right) \quad o\left(\Delta r, r_{\text {tor }}\right):= \begin{cases}1 & \text { if } \quad \Delta r<r_{\text {tor }} \\ 0 & \text { otherwise }\end{cases}
$$

In eq 5.9 we have labeled the particles inside the torus from 1 to $N_{\text {tor }}$ to simplify the notation.

Since the gyration tensor is a symmetric matrix we can diagonalize it:

$$
S=\left(\begin{array}{ccc}
\lambda_{x x} & 0 & 0 \\
0 & \lambda_{y y} & 0 \\
0 & 0 & \lambda_{z z}
\end{array}\right)
$$


FiguRE 5.13: lhs) Top view of the particles contained inside the torus at low hydration in Martini simulations at $N_{w} / N_{l}=5$. rhs) Acylindricity depending on the hydration level and presence of oil chains, hyd refers to Martini simulations and the number to $N_{w} / N_{l}$.
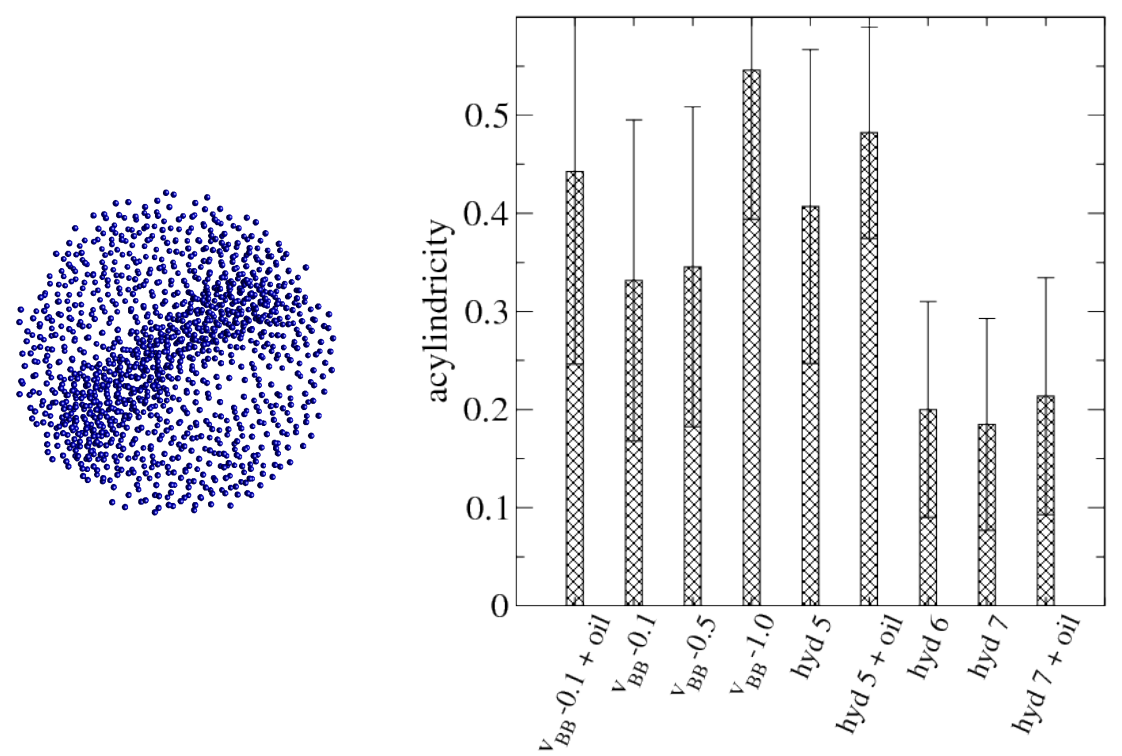

The acylindricity, $a_{c y l}$, is hence defined as:

$$
a_{c y l}=\frac{\left|\lambda_{x x}-\lambda_{y y}\right|}{\lambda_{x x}+\lambda_{y y}}
$$

We have plotted the acylindricity for different systems and quantified the influence of the hydration and the presence of oil on the expansion of the stalk (see fig $\sqrt[5.13]{ }$ ). We observe that both the oil and the reduction of hydration expands the stalk and we suggest to use the acylindricity as order parameter for the phase transition between the planar and the inverted hexagonal phase.

\subsection{1 bilayer repulsion}

The interactions between the missing water molecules and the head groups of the lipids is characterized in the parameter $v_{B B}$, which influences the depletion and the hydration force between two opposed membranes.
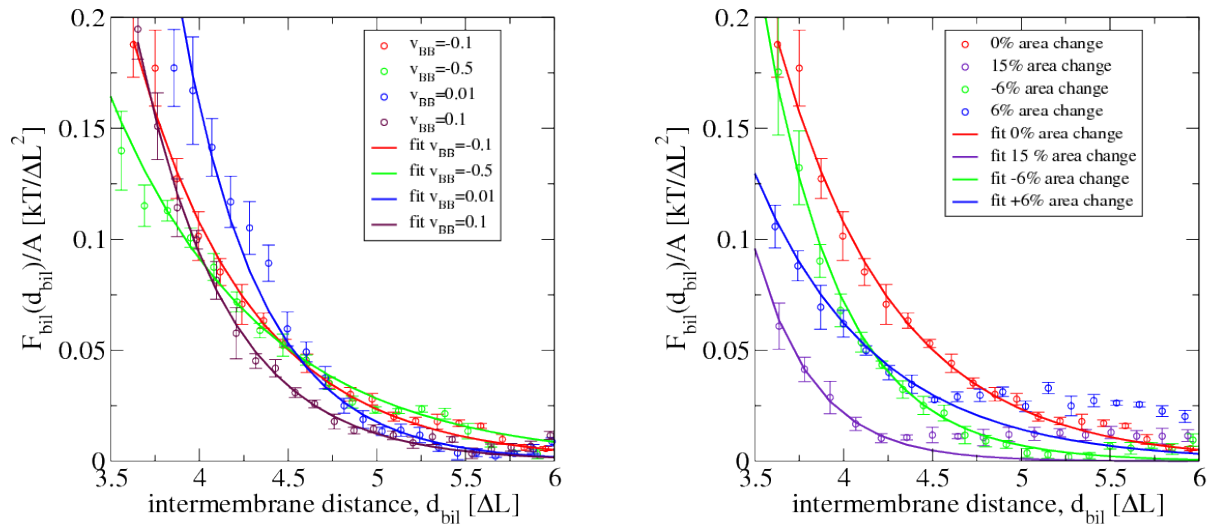

We have simulated two opposed bilayers composed by 3737 lipids each in $N P_{t} T$
Figure 5.14: Free energy versus bilayer distance. The decay length of the hydration repulsion is calculated from the exponential fit and depends on the choice of the hydrophilic virial coefficient $v_{B B}$ (lhs) or on the lateral tension (rhs). 
ensemble where we have kept fixed the normal direction: $L_{z}=14[\Delta L]$. The space is optimized to allow enough separation between the two bilayers (zero interactions) and to sample the total length of the simulation box. The intermembrane repulsion includes many different contributions: the membrane fluctuations, the depletion forces, and the enthalpic energy of the water/head groups interactions Leikin et al. (1993); Berkowitz and Raghavan (1994)]. In an coarse-grained implicit solvent model we can not correctly reproduce the complex interactions between the polar water and the head groups of the lipids but we can investigate the dependence of the repulsion energy on the lipid compositions, the boundary conditions and the hydration level. The comparisons with experiments and simulations is hence important to point out which contributions are fundamental in the calculation of the repulsion energy.

Thanks to the large scale of the system calculated, the absence of solvent and the softness of the interactions we sample the free energy landscape by thermal fluctuations. In particular, the solvent-free model does not face difficulties related to equilibrating the solvent between the opposed bilayers with the surroundings.

We calculate the probability distribution of finding the membranes at a certain bilayer separation, $d_{b i l}$, which is the distance between the head groups of the two facing leaflets of the two bilayers.

\begin{tabular}{ccc|ccc}
$v_{B B}$ & $d_{0}$ & $E_{0} / A$ & $(a-\bar{a}) / \bar{a}$ & $d_{0}$ & $E_{0} / A$ \\
\hline$v_{B B}=0.1$ & 0.49 & 0.75 & $-6 \%$ & 0.38 & 0.43 \\
$v_{B B}=0.01$ & 0.45 & 0.84 & $0 \%$ & 0.49 & 0.75 \\
$v_{B B}=-0.1$ & 0.65 & 0.58 & $+6 \%$ & 0.68 & 0.49 \\
$v_{B B}=-0.5$ & 0.85 & 0.36 & $+15 \%$ & 0.33 & 0.91 \\
& & & & & \\
composition & $d_{0}$ & $E_{0} / A$ & composition & $d_{0}$ & $E_{0} / A$ \\
\hline PC & 0.65 & 0.65 & PE & 0.71 & 0.55 \\
PC+oil & 0.70 & 0.52 & PC+chol & 0.68 & 0.39 \\
& {$[\Delta L]$} & {$\left[k_{B} T / \Delta L^{2}\right]$} & & {$[\Delta L]$} & {$\left[k_{B} T / \Delta L^{2}\right]$}
\end{tabular}

TABLE 5.4: Decay lengths and energetic prefactors from the interpolation of the free energy of the bilayer repulsion at small intermembrane distances. The upper block represents bilayer composed by single tail lipids with 8 hydrophobic and 2 hydrophilic beads. In this block it is shown the dependence of the bilayer repulsion on the virial coefficient $v_{B B}$ and lateral

The ensemble distribution of the intermembrane distance is calculated recovering the dependence of the energy $F$ by the bilayer separation $d_{b i l}$ : The intermembrane distance is calculated either between the upper leaflet of the bottom bilayer and the lower leaflet of the top bilayer or between the upper leaflet of the top bilayer and the lower leaflet of the bottom bilayer considering the periodic image convection. Only the shorter distance among the two is chosen.

$$
\begin{aligned}
P\left(d_{b i l}\right) & =\int \mathrm{d} r^{N} e^{-H\left(r^{N}\right)} \delta\left(d_{b i l}-\left(z_{1}^{c m}-z_{2}^{c m}\right)\right) \\
F_{b i l}\left(d_{b i l}\right) & =-\ln P\left(d_{b i l}\right)=E_{0} e^{-\frac{d_{b i l}}{d_{0}}}
\end{aligned}
$$

We have calculated the position of the upper monolayer of the lower membrane, $z_{1}^{c m}$, and the position of the lower monolayer of the upper membrane, $z_{2}^{c m}$, calculating the center of mass of the chains contained in the respective monolayer. To improve the sampling we have divided the membrane in $6 \times 6$ patches, this patch size furnishes a stable definition of the monolayer height (the average is over tens of chains) and shows more fluctuations. The energetic values obtained from the probability distribution of the bilayer distance are then divided by the patch area.

From the data in the table (see table:5.4) we conclude that the virial coefficient $v_{B B}$ is a control parameter for the hydration forces between two opposed bilayers. Decreasing $v_{B B}$ we observe that the repulsion energy, $E_{0} / A$, decreases and the decay length, $d_{0}$, increases (the membrane are more likely to stay closer). We therefore see that $v_{B B}$ as a direct influence on the repulsion energy.

Changing the lateral tension we control as well the repulsion energy between the bilayers. Looking at the table (see table:5.4) we realize that the behaviour is not the tension. In the lower block it is shown the dependence of the bilayer repulsion on the lipid composition.

$z_{1}^{c m}-z_{2}^{c m}$ distance between the center of mass of the chains of the two facing leaflets, $d_{0}$ decay length of the intermembrane repulsion, $E_{0}$ energetic prefactor. 
Figure 5.15: Density plot of the stalk at different hydrations. The change of the hydrophilic virial coefficient (lhs) $v_{B B}=-0.1$, rhs) $\left.v_{B B}=-0.5\right)$ changes equilibrium separation between the two leaflets.

Figure 5.16: Cross section of a fusion process between two membranes at low hydration $\left(v_{B B}=-0.8,(a-\bar{a}) / a=15 \%\right)$. A stalk is initially created between the two opposed bilayers (lhs). A pore is created in the lower bilayer and the stalk expand radially around it (rhs). The fusion pore has been formed after the creation of a pore on the upper bilayer (bottom). The violet surface represents the isoline of the density of hydrophobic beads. The figures represent an average over six snapshots.
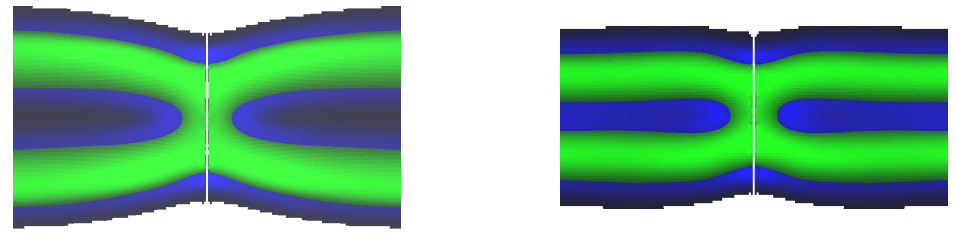

one expected, the bilayer repulsion does not decrease upon lateral stretching. We should consider that the total free energy is:

$$
F_{b i l}\left(d_{b i l}, \Delta a\right)=F_{r e p}\left(d_{b i l}\right)+F_{c o m}(\Delta a)=F_{r e p}\left(d_{b i l}\right)+\frac{k_{c o m}}{2} \frac{(a-\bar{a})^{2}}{\bar{a}^{2}}
$$

Applying this correction we obtain:

- $\left(d_{0}=0.73, E_{o} / A=0.48\right) @+15 \%$

- $\left(d_{0}=0.74, E_{o} / A=0.51\right) @-6 \%$

- $\left(d_{0}=0.68, E_{o} / A=0.53\right) @+6 \%$

and we see that the tension differences reduce the bilayer repulsion. If we shrink the membrane area $(+6 \%)$ the protrusions increase the probability of a contact between the opposed bilayers and the patch-based definition of the intermembrane distance is in this case not consistent.

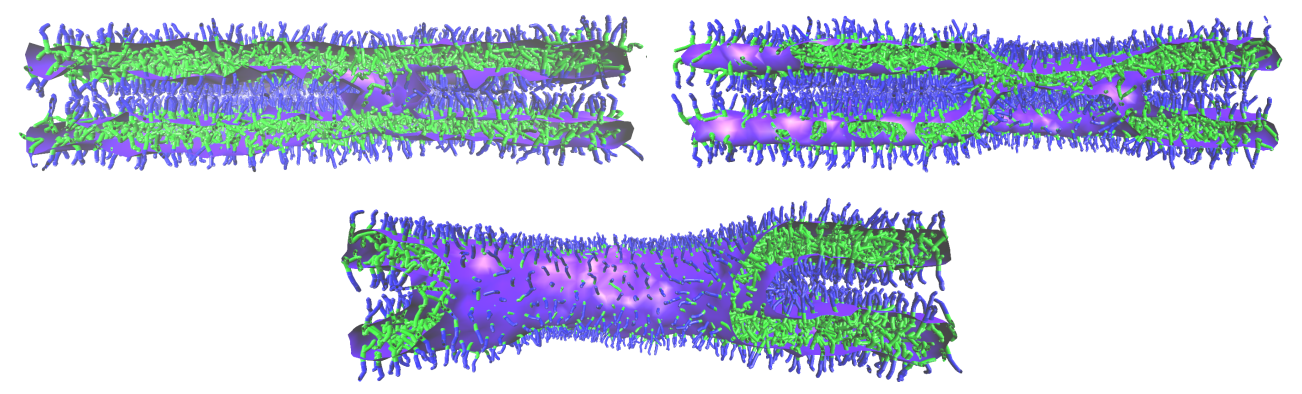

Referring to the literature we compare the calculated decay lengths with the values presented in the literature. Specifically we address to the decay length, $d_{0}$, for a POPC lipid system is for Martini simulations $d_{0}=0.26[\mathrm{~nm}]$ [Smirnova (2012)] and for experiment $d_{0}=0.22[\mathrm{~nm}]$ Aeffner et al. (2009)] which compared to the values of the plot (see fig 5.14) suggest us to set $v_{B B}=0.1$ for the comparison with the experiments. Between two opposed membranes we observe adhesion and lipid mixing at low hydrations. Controlling the lateral tension of the simulation box we have observed a fusion event steered from a stalk formation between two bilayers (see fig 5.16). After the formation of the stalk a pore is created at its lower end. The pore successively expands pulled by the lateral tension. Successively another pore is created in the opposite bilayer and the stalk start elongating sealing the rims of the two pores together. The last picture of figure 5.16 shows the completed fusion between the two opposed bilayers.

\subsection{2 conclusions}

We have shown that with our model we are able to create PC and PE lipid types, and we have developed two methods to describe the position and size fluctuations of circular and linear stalks. From the size fluctuations we could calculate the stiffness of the linear and circular stalk for different lipid compositions. We observe that the addition of oil chains expands the stalk trunk but does not influence the indentation 
on the lower and upper end. We have compared the solvent-free model with explicit solvent model and we study how the head-group interactions can mimic the hydration between the bilayer. We quantify how bilayer repulsion changes under hydration and lateral tension.

Controlling the hydration level in different models we have identified a transition between inverted hexagonal/rombohedral/lamellar phases. Between two opposed membranes under lateral tension, pore and stalk formation are competing phenomena. The hydration between the two bilayers enhance the stalk formation as initial stage of the fusion process. We have observed that already by a $15 \%$ area increase and a virial coefficient of $v_{B B}<-0.8$ the stalk formation was preferred to the pore formation and consequent rupturing of both membrane. 
CHAPTER 5. STALK DYNAMICS

conclusions AND MORPHOLOGY 


\section{Chapter 6}

\section{inclusion interactions}

Transmembrane peptides locally induce important membrane modifications that might have as extreme effect the stabilization of pores in tensionless membranes and cause the cell's death (apoptosis). These processes are collective phenomena that we study by solvent-free coarse grained models.

Based on the hydrophobic mismatch and the surface energy of the peptide we identify the contact angle between the peptide and the membrane. For a fixed contact angle and hydrophobic mismatch the thickness profile shows an under-/over-shoot compensated by an over-/under-shoot above or below the bulk thickness. The lipid-mediated interactions between two peptides are quantified by placing the peptides at fixed distances and calculating the thickness profile on the line connecting the two peptides. From the thickness profile we extract the interpeptide distance where the thinning reaches its maximum. We study the superposition of the effects in the thickness profile for inclusions sitting at the vertices of regular polygons. To explore the large parameter space we compare the results of the simulations with the numerical solution of the Helfrich Hamiltonian of two coupled elastic sheets.

The particle-based simulations allows us to study the modifications of the chain conformation and we show the radial profiles of notable chain modifications. From those radial profiles we notice that beyond the enrichment zone, the average density is slightly lower than the bulk one, the mean separation of the lipids is larger and the membrane is prone to pore formation.

\section{1 minimal models for thinning of lipid membranes by transmembrane proteins}

Fluid membranes are mechanically extremely resistant and function as a barrier between the cytosol and the extracellular liquid [Seifert et al. (1991); Miao et al. (1991, 1994); Kocun et al. (2011, 2010)]. The transport of compounds inside and outside the cell is regulated by a leakage mechanism of transmembrane proteins. These proteins are mainly composed by a sequence of aminoacids ordered in $\alpha$-helices or $\beta$-sheets. Some of the residues composing the peptide may be apolar and sit in contact with the head groups of the lipids and orient the axis of the protein normal to the bilayer plane Luckey (2008)]. With the term protein we want to describe a large class of transmembrane inclusions (or gramicidin channel Helfrich and Jakobsson (1990b); Huang (1986a); Harroun et al. (1999); Helfrich and Jakobsson (1990a)]) that perturb the surrounding membrane. The perturbation induced by a single locally protein weakens the mechanical resistance of the membrane and the combined effect of many proteins can stabilize a pore. We distinguish between double-sided proteins (one side is hydrophilic) that stabilize barrel stave pores (the proteins sit at the rim) and single sided proteins that stabilize toroidal pores (the proteins do not form the pore rim) 
Yang et al. (2001)]. Double-sided peptides (like magainin, melittin or influenza virus peptides) form spontaneously bundles inside the membrane and can stabilize a barrel stave pore Risselada et al. (2012); Illya and Deserno (2008)].

The stabilization of a pore is a collective phenomenon where the transmembrane proteins do not directly interact between themselves but induce a long-range modification of the lipids Goulian et al. (1993); Kim et al. (1998); Prost and Bruinsma (1996)]. The protein membrane interactions are a well studied topic in theory [Huang (1986b); Helfrich and Jakobsson (1990a); Partenskii and Jordan (2002); Nielsen et al. (1998); Marčelja (1976)], in experiments Leikin et al. (1994); Leikin and Parsegian (1994); Leikin et al. [1995)] and simulations $\operatorname{May}(2002) ;$ West et al. (2009); Niemelä et al. (2010); Venturoli et al. (2006, 2005); Reister and Seifert (2005); Sintes and Baumgärtner (1997); Fattal and Ben-Shaul (1993)]. On the other hand the superposition of the effect of the single protein is not well studied Schmidt et al. (2008); Aranda-Espinoza et al. (1996); Harroun et al. (1999)] and should largely deviate from the linear response theory. To this end, we work towards studying the local perturbation in the macroscopic (thickness and density) and in the microscopic (chain diffusivity, stretching and orientation) scales for single peptides. We investigate the combined effect analyzing the interpeptide distance where the weakening of the membrane reaches its maximum. We analyze the combined effect of peptides by constructing a cluster of peptides positioned on the vertices of regular polygons distant from the center by the weakening distance. We observe that clusters with at least five proteins leads to the stabilization of pores.

The parameter space to explore the problem of superposition is large as it considers three parameters for each inclusion (radius, hydrophobic mismatch and surface energy), the number or peptides, and the distance between them. We present a continuum model and explore its solutions with the results of the simulation to speed up the computation by a factor of ten thousand allowing us a fine exploration of the parameter space. We show the compatibility and the break down of this mapping in case of the pore stabilization.

\section{2 soft description}

A detailed modeling of the inclusion is done by connecting a cluster of monomers by harmonic springs (see fig 6.1). This description eases the definition of different patterns and shapes (useful for the stabilization of barrel stave pores).

Figure 6.1: lhs) Sketch of a cluster of tethered monomers, rhs) surface energy of the peptide depending on the number of beads. We choose the number of beads per area marked by the red circle, $N_{b} / A=38$.
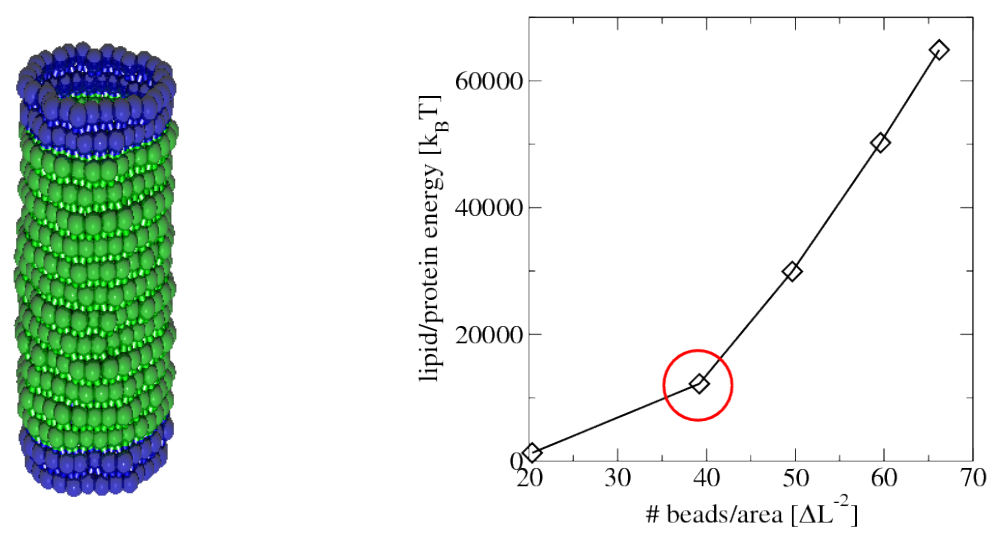

The surface energy of the cluster is connected with the number of monomers describing the cylinder and the fluctuation of the shape with the strength of the harmonic springs 
connecting the beads.

$$
U_{i j}=200 k_{s p}\left(r_{i j}-\bar{r}_{i j}\right)^{2}
$$

The protein is composed by coaxial rings of monomers connected by harmonic springs (see fig 6.1), the odd rings have a $10 \%$ increase in the radius to improve the resistance to lateral compression. We connect only neighboring beads, each bead is connected with the two neighboring beads on the lateral direction and the two on the normal direction. The strength of the harmonic potential controls the shape fluctuations of the peptide and we set the prefactor to $200 k_{s p}$ to conserve the cylindrical shape under the pressure of the lipids. The axis of the soft inclusion is obtained interpolating all positions of the monomers.

\section{3 parameter space}

The parameter space we are considering consists in the hydrophobic mismatch, radius and surface energy for every peptide, an additional degree of freedom is the interpeptide distance and the number of peptides sitting at the vertices of regular polygons: $\left(r_{p e p}, h_{p e p}, \epsilon_{p e p}, r_{p e p}^{I I}, N_{p e p}\right)$.

Depending on the hydrophobic mismatch and on the lateral radius of the peptide the protein may fluctuate around an orientation angle tilted with respect to the normal. To calculate the profile we position first the inclusion in the center of the frame and rotate the system so that the peptide's orientation axis lies on the plane $(x, z)$.

If the peptide is tilted we loose the radial symmetry since the we can not project the bead positions neither on the normal direction nor on the peptide's axis without giving a distorted representation of the membrane. Moreover, the tilting angle adds an additional degree of freedom that we want to neglect in exploring the large parameter space we are considering.

In the soft description we add an external harmonic potential oriented normally to the surface of the membrane and recover the radial symmetry:

$$
U_{\text {ext }}\left(r_{i / p e p} ; r_{p e p}\right):=200 k_{s p}\left(r_{i / p e p}-r_{p e p}\right)^{2} \quad r_{i / p e p}^{2}:=\left(x_{i}-x_{p e p}\right)^{2}+\left(y_{i}-y_{p e p}\right)^{2}
$$

No constrain is used on the normal direction. The external potential is meant as well to be used as umbrella potential to calculate the potential mean force between two peptides.

Above a certain value of the hydrophobic mismatch and the surface energy the membrane bulges around the peptide and perturbs the radial profiles. The profiles we present are below this threshold.

\section{4 continuum model}

Since the solution of a differential equation on the lattice is about ten thousand times faster than a single simulation we compare the results obtained with the solution of the elastic model to be able to explore the large parameter space.

We refer to the continuum model for bilayer membranes defined by West et al. (2009); Branningan and Brown (2006, 2007)] where the membrane is described as two thick coupled elastic sheets. Each elastic sheet has an energy cost for bending and stretching and the elastic coupling is described as an harmonic potential. The free energy of the model is:

$$
\begin{aligned}
F & =\frac{1}{2} \int \mathrm{d} x \mathrm{~d} y\left(k_{b e n}\left(\nabla^{2} d_{s}(x, y)\right)^{2}+4 k_{b e n} c_{0} \nabla^{2} d_{s}(x, y)+4 \frac{k_{e l}}{\bar{d}^{2}} d^{2}(x, y)\right. \\
& \left.+\gamma^{\prime} d_{s}(x, y) \nabla^{2} d_{s}(x, y)\right)
\end{aligned}
$$

$r_{i j}$ is the distance of the tether between the particle $i$ and the particle $j, \bar{r}_{i j}$ is the equilibrium distance of the tether, $k_{s p}$ elastic coupling between the monomers in the chains. $k_{\text {ben }}$ is the bending rigidity, $\gamma^{\prime}$ controls the asymmetry between the bilayer (it is connected with the spontaneous curvature, $k_{e l}$ the elastic coupling. 
The term $\gamma^{\prime}$ is connected with the spontaneous curvature, $c_{0}$, with this relationship Branningan and Brown (2006)]:

$$
\gamma^{\prime}:=4 k_{\text {ben }}\left(c_{0}-\frac{\partial c_{0}}{\partial A} A\right)
$$

The Hamiltonian (eq:6.3) recalls the Hamiltonian already introduced in (eq 4.32 where we have excluded the protrusion contributions (dependent on the coupling terms $\gamma_{\lambda}$, $k_{\lambda}$ ) that can not be described in continuum models. In 4.32 we have defined $h(x, y)$ as the height of the membrane at the point $(x, y)$ and $d(x, y)$ as the thickness of the membrane at the same point. We have changed the coordinate system and disregarded the fluctuations over the average $h(x, y)$. In $(6.3)$ we consider $d_{s}(x, y)$ as the position of the intersection between the hydrophobic and the hydrophilic beads of the upper leaflet (the lower leaflets is symmetric to the upper one). The transformation between $d(x, y)$ and $d_{s}(x, y)$ is simply:

$$
d_{s}(x, y)=d(x, y)-\bar{d}
$$

This simple change allows us to describe the membrane modification by a single function, $d_{s}(x, y)$, and $d_{s}(x, y)=0$ corresponds to the ground state of the energy 6.3. Every modification of the surface $d_{s}(x, y)$ from the ground state gives a energetic contribution in terms of bending, stretching and elastic coupling.

Figure 6.2: lhs) Sketch of the numerical solution of the Euler-Lagrange equations in 2 d. The bonds represent the connections between the lattice points. The blue and red points fix the boundary conditions. The violet points represent the thickness profile calculated from the simulation on the same grid discretisation as the numerical solution.

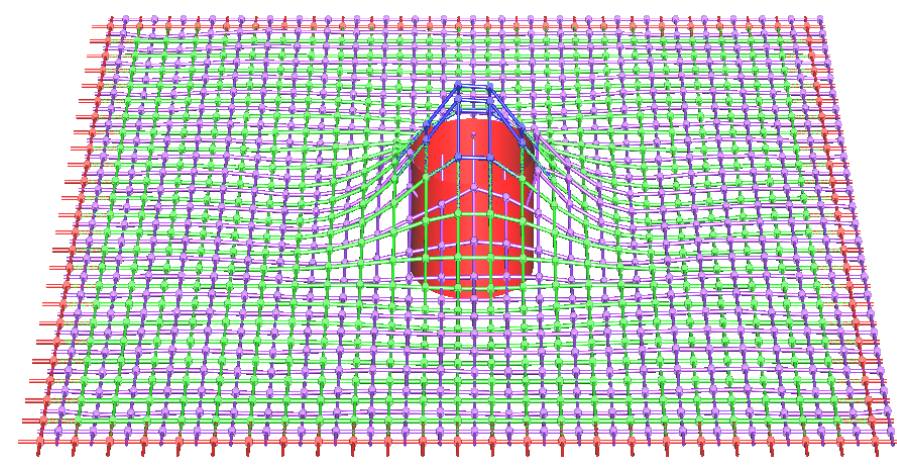

\section{Euler-Lagrange equations}

We calculate the Euler-Lagrange equation from the free energy expressed in (see eq 6.3 ) and obtain [West et al. (2009)]:

$$
\frac{\delta F}{\delta d_{s}}=0 \quad\left(k_{b e n} \nabla^{4}+\gamma^{\prime} \nabla^{2}+k_{e l}\right) d_{s}(x, y)=0
$$

We solve the differential equation (eq6.7) in different steps. We consider first the Cartesian one dimensional problem.

$$
D_{x} d_{s}(x)=0 \quad D_{x}:=k_{b e n} \nabla_{x}^{4}+\gamma^{\prime} \nabla_{x}^{2}+k_{e l} \quad \nabla_{x}^{2}:=\frac{\mathrm{d}^{2}}{\mathrm{~d} x^{2}}
$$

To calculate the solution of the linear differential equation we search for a solution of the kind $\exp (\lambda x)$ and write the characteristic equation:

$$
k_{b e n} \lambda^{4}+\gamma^{\prime} \lambda^{2}+k_{e l}=0 \quad \lambda_{ \pm \pm}= \pm \sqrt{\frac{-\gamma^{\prime} \pm \sqrt{\gamma^{\prime 2}-2 k_{b e n} k_{e l}}}{2 k_{b e n}}}
$$

which gives the solution:

$$
d_{s}(x)=c_{1} e^{\lambda_{++} x}+c_{2} e^{-\lambda_{++} x}+c_{3} e^{\lambda_{+-} x}+c_{4} e^{-\lambda_{+-} x}
$$


We see that the following boundary conditions:

$$
d_{s}\left(r_{p}\right)=h_{p} \quad d_{s}^{\prime}\left(r_{p}\right)=\tan \theta_{c} \quad d_{s}\left(L_{x}\right)=0 \quad d_{s}^{\prime}\left(L_{x}\right)=0
$$

are sufficient to determine the parameters $c_{i}$.

Second we calculate the radial solution where the Laplacian operator turns into:

$$
D_{r} d_{s}(r)=0 \quad D_{r}:=k_{b e n} \nabla_{r}^{4}+\gamma^{\prime} \nabla_{r}^{2}+k_{e l} \quad \nabla_{r}^{2}:=\frac{1}{r} \partial_{r}\left(r \partial_{r}\right)
$$

The radial solution of the equation $(6.7)$ is West et al. (2009)]:

$$
d_{s}(r)=c_{1} J_{0}\left(\lambda_{++} r\right)+c_{2} Y_{0}\left(\lambda_{++} r\right)+c_{3} J_{0}\left(\lambda_{+-} r\right)+c_{4} Y_{0}\left(\lambda_{+-} r\right)
$$

where the $\lambda$ coefficients are the same calculated for the one dimensional Cartesian problem (eq 6.9 ) and we use the same boundary conditions (eq 6.10).

Third we define the differential operator in terms of partial derivatives in Cartesian coordinates:

$$
D_{c} d_{s}(x, y)=0 \quad D_{c}:=k_{b e n} \nabla_{c}^{4}+\gamma^{\prime} \nabla_{c}^{2}+k_{e l} \quad \nabla_{c}^{2}:=\left(\begin{array}{cc}
\partial_{x}^{2} & 0 \\
0 & \partial_{y}^{2}
\end{array}\right)
$$

The solution of partial differential equations is a complex problem that uses the LaxMilgram theorem to show the existence and uniqueness of a weak solution to a given boundary problem Babuška (1971)]. To avoid the complexity of treating the mathematical problem we test the solution of the discretized two dimensional problem with the analytical radial solution. We create a two dimensional square lattice, we discretized the operators and we put the following boundary conditions:

$$
\begin{aligned}
\left\{d_{s}(x, y)=h_{p} \mid x^{2}+y^{2}=r_{p}\right\} & \left\{d_{s}^{\prime}(x, y)=\tan \theta_{c} \mid x^{2}+y^{2}=r_{p}\right\} \\
\left\{d_{s}(x, y)=0 \mid x=L_{x} \vee y=L_{y}\right\} & \left\{d_{s}^{\prime}(x, y)=0 \mid x=L_{x} \vee y=L_{y}\right\}
\end{aligned}
$$

We calculate the numerical solution of the Euler-Lagrange equations for a square lattice in two dimensions (see fig:6.2) using finite differences (see appendix).
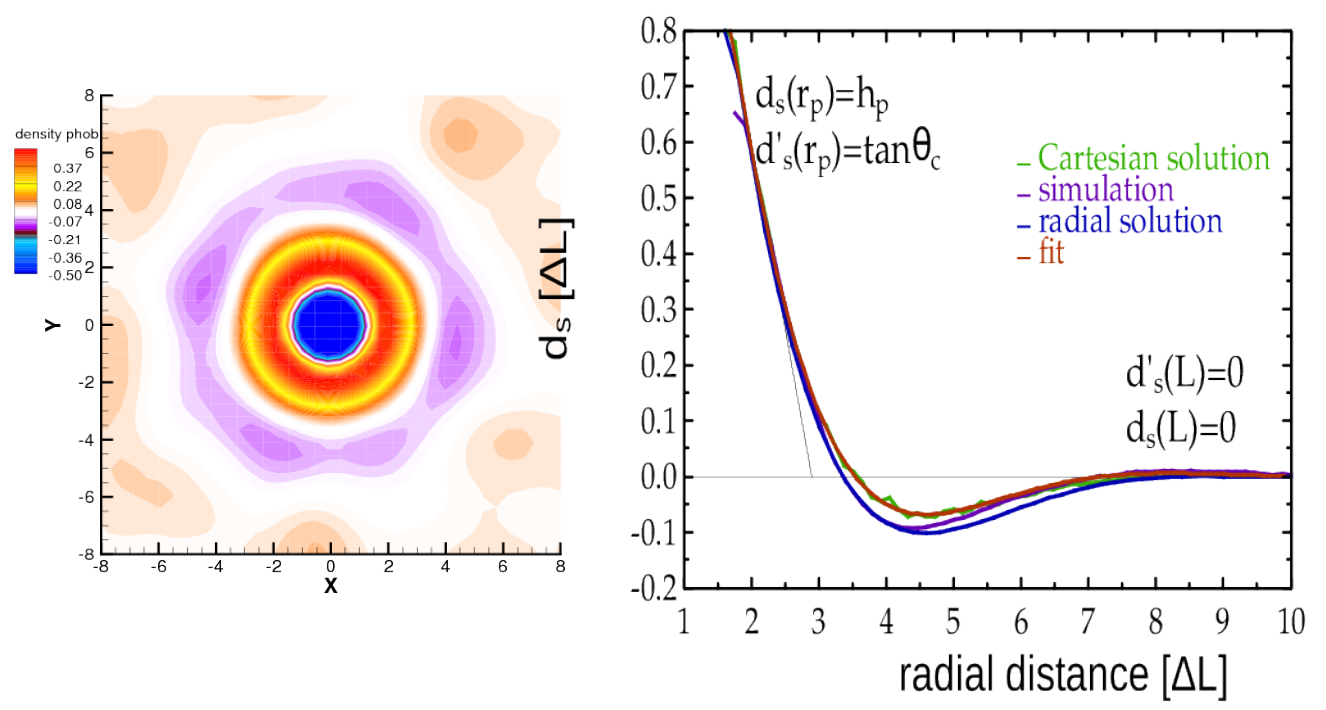

Figure 6.3: lhs) thickness profile in Cartesian coordinates. rhs) comparison between the thickness profiles. The green and the purple lines represent the radial average from the Cartesian thickness profiles form the simulations (green line) and from the $2 \mathrm{~d}$ numerical solution of (eq 6.15) (red line). The red line is obtained by fitting with the simulation data with the function $A e^{-x / \lambda} \sin (f x) / x$, the blue line shows the numerical solution in cylindrical coordinates.

To map the parameter of the differential equation into the simulated system we rescale the constants:

$$
\left(\nabla^{4}+\frac{\gamma^{\prime}}{k_{\text {ben }}} \nabla^{2}+\frac{k_{e l}}{k_{\text {ben }}}\right) d_{s}(x, y)=0
$$


$A$ amplitude, $\xi$ correlation length and $f$ the oscillation wave vector.
We refer to a lipid bilayer using the values calculated in the previous chapter (see table 4.3 and use the following values:

$$
\left(\nabla^{4}+0.5 \nabla^{2}+0.57\right) d_{s}(x, y)=0
$$

If the peptide sits in the middle of the lattice we recover radial symmetry around the peptide center. We reduce the two dimensional solution into the radial one integrating over the angle and we superimpose the two solutions on the same plot (see fig 6.3).

The thickness profile obtained from the simulations is the result of the radial average of the density profile. We have rescaled the density profile by the ratio $\bar{d} / \bar{\rho}$ and shifted the value of the bulk thickness, $\bar{d}$, to the ground line:

$$
d(r)=\bar{d}\left(\frac{\rho_{A}(r)}{\bar{\rho}}-1\right)
$$

The thickness profile obtained from the numerical calculation is the radial average profile of the height of the grid points around the inclusion.

The agreement between the analytical solution, the reduced two dimensional solution and the thickness profile obtained from the simulations (see fig 6.3) furnishes a satisfying argument about the reliability of the two dimensional solution.

We want to express the solution using a physical analogy with the damped harmonic oscillator in far field approximation in cylindrical coordinates:

$$
d_{s}(r)=A e^{-r / \xi} \frac{\sin (f r)}{r}
$$

Fitting the solution of the numerical calculation or the simulation we can extract the correlation length and the oscillation wave vector of the protein lipid interaction: $\xi=2.25[\Delta L]$ and $f=0.88\left[\Delta L^{-1}\right]$ (see fig:6.3).

\section{5 simulation description}

We have created different hydrophobic inclusions in a cylindrical cavity created in the membrane. The membrane patch simulated is at least $[29 \Delta L]$ per side to avoid any boundary effect and the simulation time is over $500\left[t_{s}\right]$. For every simulation we have ignored the equilibration steps and calculated the radial profiles described in the following sections.

\section{6 macroscopic scale}

The first information we learn about the local modification around a transmembrane protein is the change in the membrane thickness.

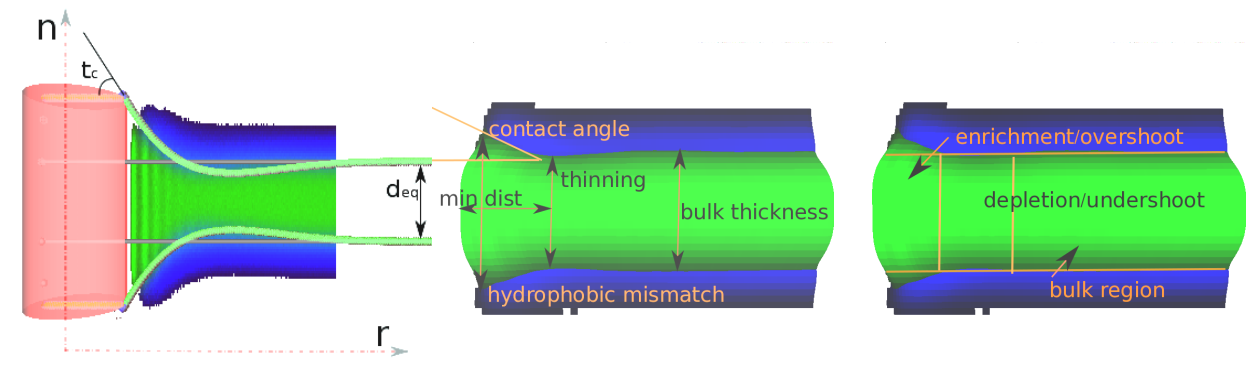

From the pictures (see fig 6.4) we can see that the positive hydrophobic mismatch of the protein causes the membrane leaflets to separate until reaching the two borders
Figure 6.4: Radial density profiles and the identification of three different zones. lhs) comparison between the thickness profile obtained in the simulation and the calculated one. The green region represents the density of the hydrophobic beads, the blue the density of the hydrophilic, the brighter is the color the denser is the region. 
of the protein defining a contact angle $\alpha_{\text {cont }}$ at the hydrophobic/hydrophilic/inclusion contact point. The thickness decreases towards its bulk value showing in some cases an undershoot below the bulk value. In these cases we can define the characteristics of the thickness profile, namely the contact angle $\alpha_{\text {cont }}$, the maximum thinning $d_{\text {max }}$ and the position of the minimum $r_{\text {min }}$. As in the simulated system we observe (see fig 6.4 that after the decrease of the profile a dip is created and the thickness of the membrane restores slowly to the equilibrium value, yellow line.

\section{7 thickness characteristics}

We have calculated the characteristics of the thickness profile depending on the parameters of the model (mainly: the radius, the hydrophobic mismatch and the surface energy, see fig 6.5.

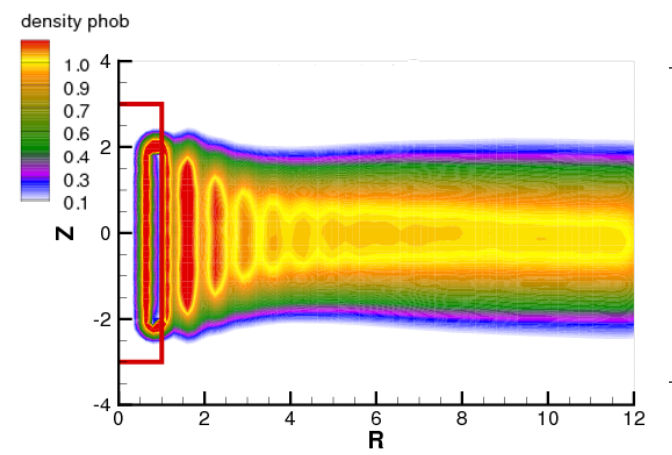

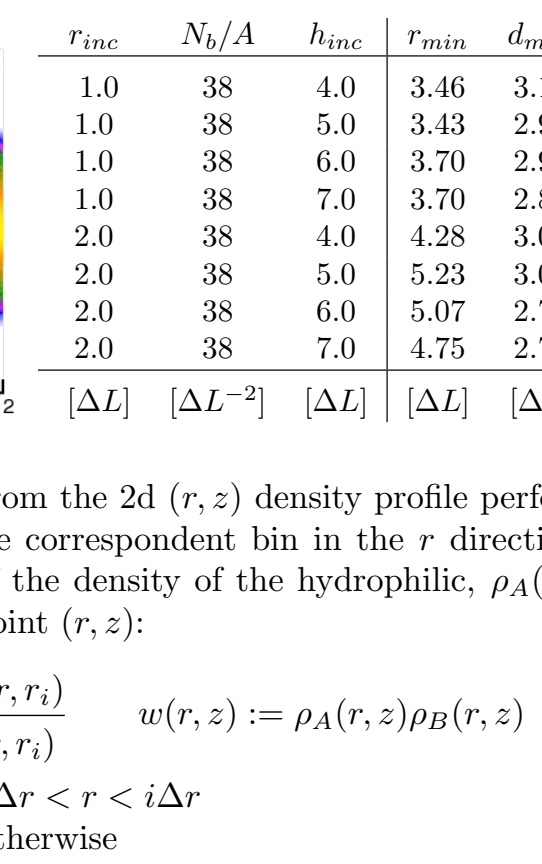

The resulting profile is the average between the one on the upper side minus the one on the lower side.

In case of Cartesian symmetry the thickness is calculated from the density profile of the hydrophobic beads:

$$
d_{s}(x, y)=\frac{\bar{d}}{\bar{\rho}} \rho_{A}(x, y)
$$

Since the melt is almost incompressible both definitions of the thickness profile are compatible.

To extract the contact angle we linearly interpolate the thickness profile close to the border with the peptide. We extract the position of the minimum and the maximum thinning interpolating with a parabola the thickness profile close to the local minimum. The continuum model allows us to explore with an enhanced discretisation the influence on the membrane deformation by the model parameters. We create a protein of radius $1[\Delta L]$ and varying the contact angle and the hydrophobic mismatch we solve the Euler-Lagrange equations (eq $\sqrt[6.13]{ }$ ) and extract the maximum thinning and the position of the minimum. We have created 400 different configurations defining 20 different contact angles (between $0^{\circ}$ and $70^{\circ}$ ) times 20 different hydrophobic mismatches (between 0 and $4[\Delta L]$ ). We have extracted the radial profiles from the numerical solutions (see fig 6.3) to obtain $r_{\min }$ and $d_{\max }$ and create the isosurfaces $\left(\theta_{c}, h_{p}, r_{\min }\right)$ and $\left(\theta_{c}, h_{p}, d_{\max }\right)$ plotted in the graph (6.6).
The thickness characteristics are: $r_{\text {min }}$ position of the minimum, $d_{\max }$ maximum thinning, $\alpha_{\text {cont }}$ contact angle.
$N_{b i n}$ number of bins, $z_{i}$ thickness profile correspondent to the bead $i, w(r, z)$ weighting function at the point $(r, z)$.
$\rho_{A}(x, y)$, density of the hydrophobic (species $A$ ) beads. 
Figure 6.6: Contour plot of the position of the minimum (lhs) and maximum thinning (rhs) depending on the height and contact angle.
Figure 6.7: Thinning of the membrane (lhs/top) and position of the minimum (rhs/top) versus interpeptide distance obtained from the simulation and the numerical calculation. lhs/bottom) Sketch of the numerical solution, the two peptides are represented by the boundary conditions (blue points), rhs/bottom) thickness profile around the two inclusions (in red).
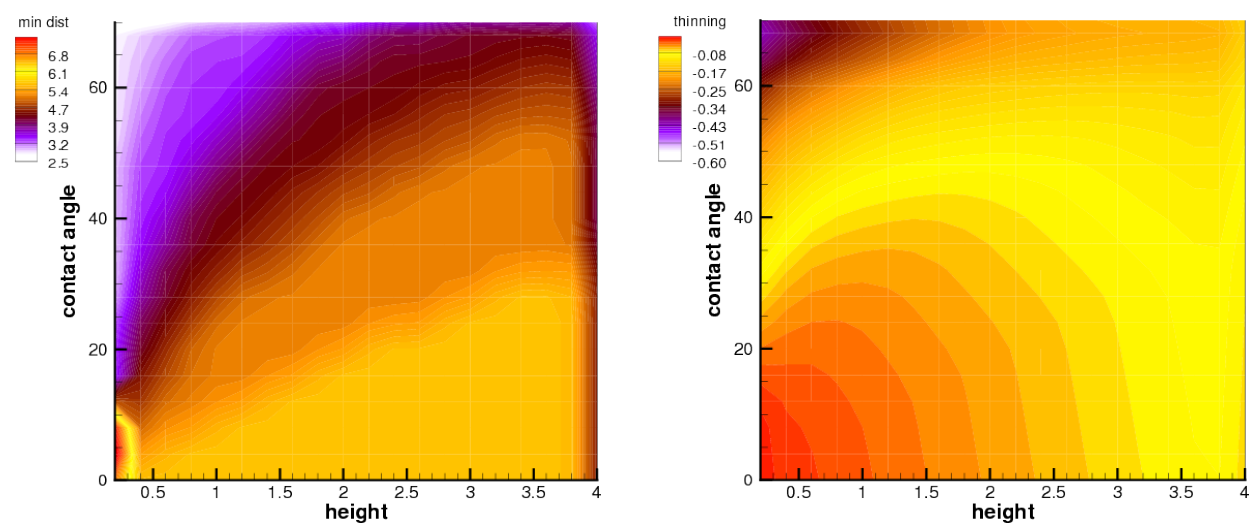

The difference between the two plots in figure 6.6 shows an interesting difference between the position of the minimum and the thinning. The hydrophobic mismatch has a considerable influence on the thinning but not on the distance from the minimum for small contact angle. For larger contact angles the role of the hydrophobic mismatch in shifting the position of the minimum is enhanced.

This analysis allowed us to explore the complicated relationship between contact angle and hydrophobic mismatch on the local membrane deformation in a fast calculation.

\section{8 superposition of effects}

To quantify the effect of the superposition of many peptides we calculate first the dependence of the thinning by the interpeptide distance.
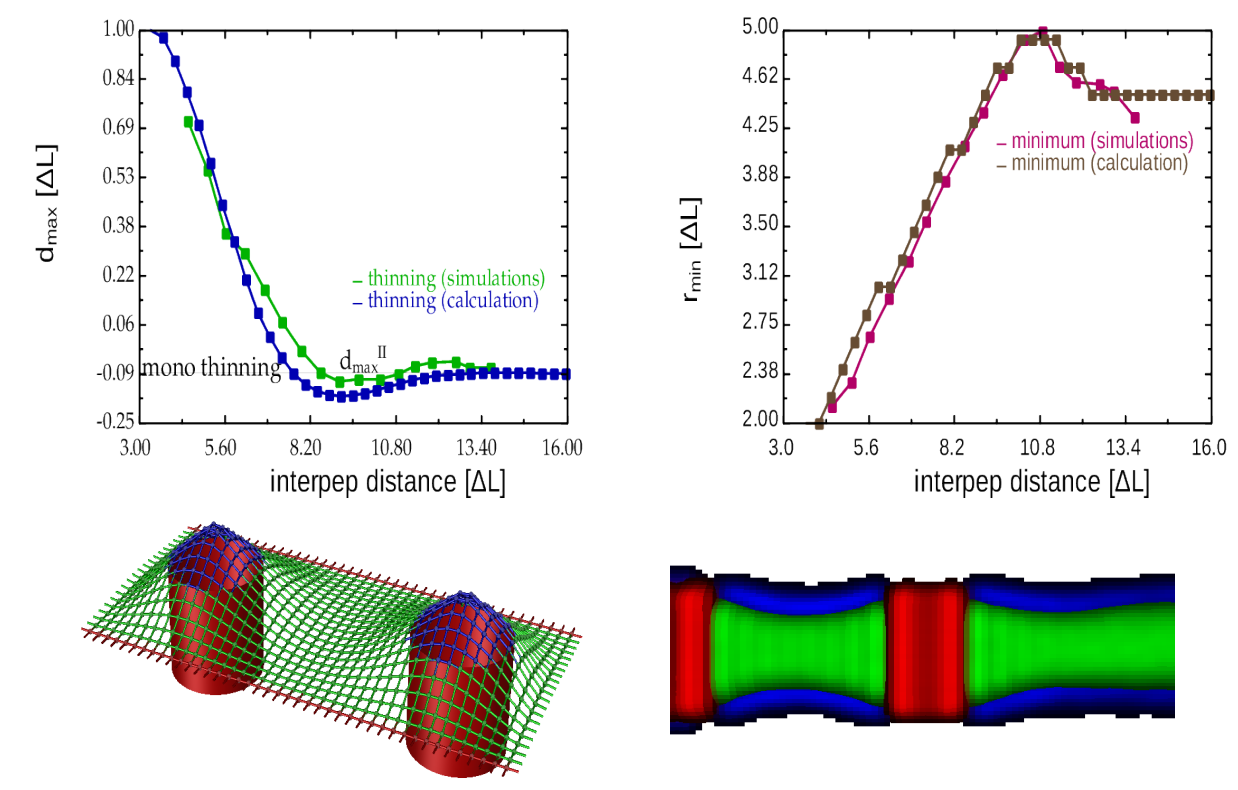

We keep two inclusions at a fixed distance and calculate the thickness profile in a slab d parallel to the line connecting the two peptides centers and as broad as the peptide diameters (see fig: 6.7 bottom right). We calculate the thickness profile from the $2 \mathrm{~d}$ density profile of the slab converting the density in thickness units. The thickness profile is directly extracted from the continuum solution considering the grid points on the line connecting the centers of the two peptides (see fig:6.7).

In the plot (see fig 6.7) we can identify the interpeptide distance where the thinning has its maximum effect and we call it the weakening length of the protein-protein 
interaction $d_{\max }^{I I}$.

Over a critical distance the combined effect of the peptides decouples and the thinning profile shows two minima instead of one (see fig 6.7).

The effect of two separated peptides superimpose and the position of the minimum $r_{\text {min }}^{I I}$ differs from the characteristic $r_{\min }$ of the single inclusion.

The data collected from the simulations are less precise since the membrane bulges around the peptides and the two peptides do not sit at the same height. The continuum model allows a finer and more precise determination of the effect of superimposition giving good qualitatively agreement.

\section{9 multiple inclusions}

We study the effect of the superposition of multiple peptides putting them on the vertices of regular polygons where the distance between the vertex and the center is the weakening length of the peptide.
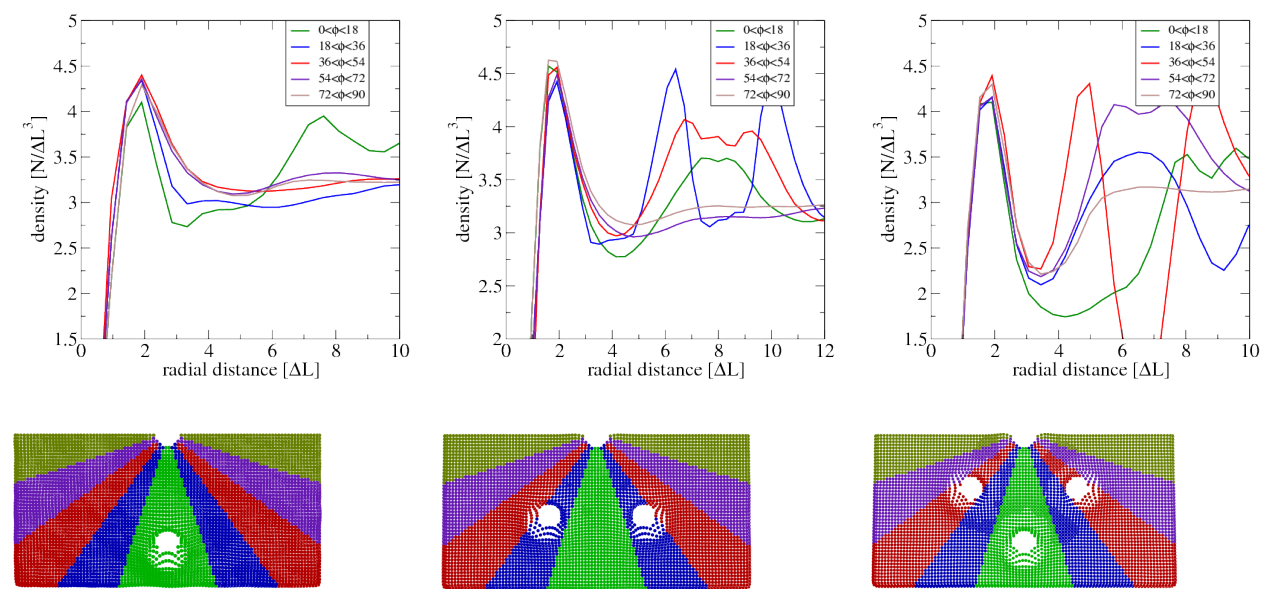

FIGURE 6.8: Thickness profiles of the angular sectors around one inclusion and correspondent sketch of the division of those angular sectors. The angular sectors are: $0^{\circ}<\phi<18^{\circ}$ (green), $18^{\circ}<\phi<36^{\circ}$ (blue), $36^{\circ}<\phi<54^{\circ}$ (red) $54^{\circ}<\phi<72^{\circ}$ (orange) $72^{\circ}<\phi<90^{\circ}$ (yellow). The columns refer respectively to two, three, and four proteins embedded in the membrane.

FiguRE 6.9: Stable pore between five transmembrane proteins.

In the plot (see fig 6.10 ) we can observe a qualitative agreement between the simulations and numerical solution. We show a contour plot of the membrane thickness in Cartesian coordinates (see fig 6.10 where the cold colors (blue and violet) are under the bulk thickness (white). The presence of the proteins is marked with the deep blue and around their borders the thickness profiles is largely above the bulk thickness (in the region marked by the warm colors: yellow, orange, and red). The effect of the superimposition is mostly visible in the middle of the polygon. The stronger difference 
FiguRE 6.10: Thickness profile around a cluster of 4 (top) and 5 (bottom) proteins obtained from the simulations (lhs) or from the continuum calculations (rhs). between the simulation results and the numerical calculation is at the exterior of the polygon, the continuum model shows a larger thinning.
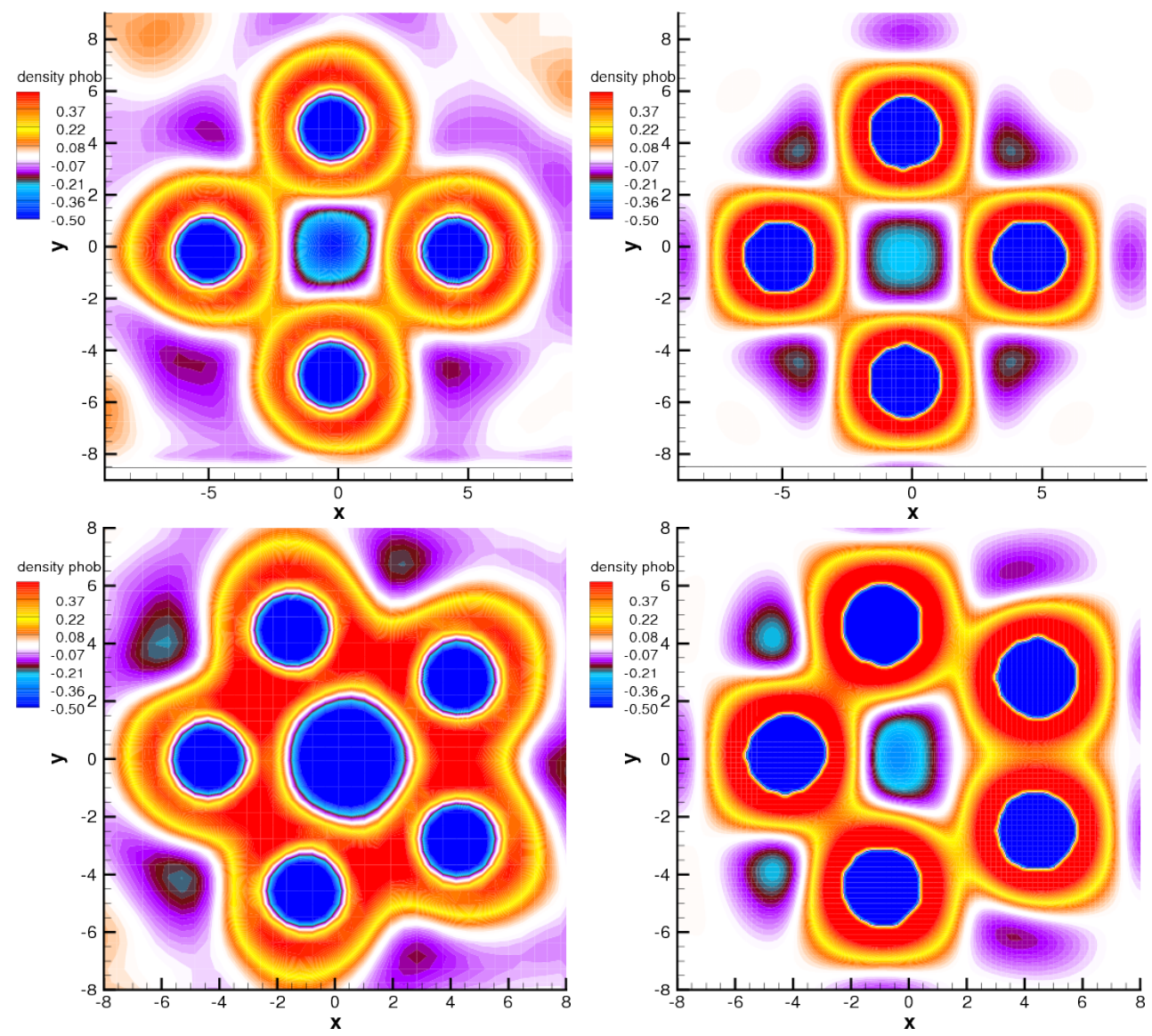

The regular square mesh is required for finite difference calculations but is not particularly suited for reproducing the boundary conditions and the circular edge of the protein.

When the peptides sit at the vertices of a regular pentagon or hexagon we observe in the simulations the formation of a stable pore in the center of the polygon (see fig 6.9). After the formation, the pore is stable even without the constrain on the peptides position (see eq 6.2 .

In this case the solution of the continuum model can not show the pore formation. A more accurate calculation of the continuum model would allow to parametrize the thickness so that below a certain threshold value the continuum model could predict pore formation under different peptide geometry.

\subsection{0 microscopic scale}

After having analyzed the local modification on the continuum level we look at the discrete change in the lipid conformations with the respect of the bulk value.

We analyze the radial profiles of different quantities (mean angle, end-to-end distance, mean separation...) and we point out the most relevant changes and characteristic lengths. We calculate at the end the diffusivity of the proteins depending on their description characteristics: $\left(r_{p e p}, h_{p e p}, \epsilon_{p e p}\right)$.

Relevant chain conformation changes around the peptide are:

- end-to-end distance

- mean separation 
- mean orientation

- mean separation

For each quantity we compare the radial profiles in the different zones comparing the characteristic lengths of the data plotted in the graph (see fig 6.11). The density of chains is normalized by the volume of the portion considered while the other quantities are normalized by the number of chains and hence uncorrelated from the densities.

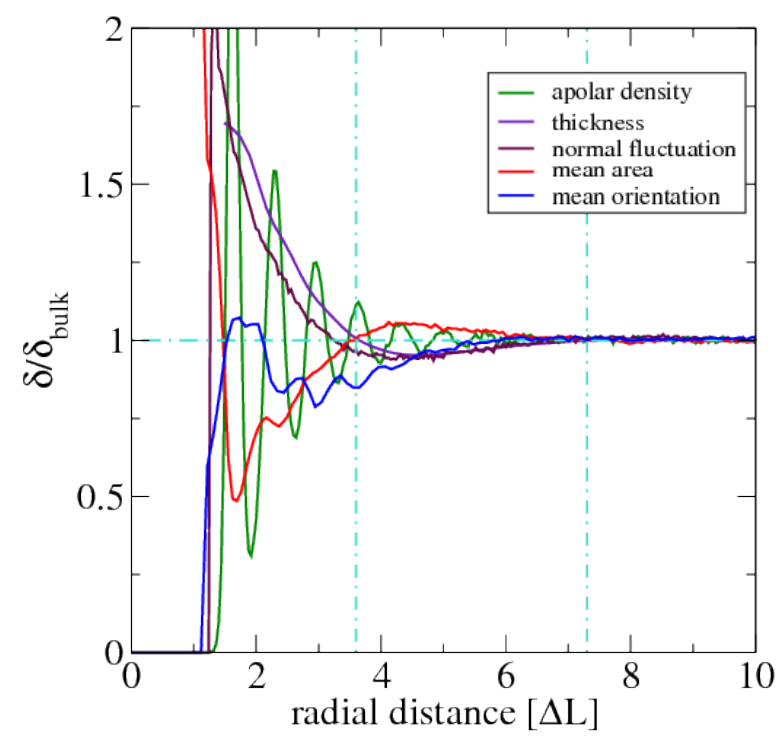

end-to-end distance

In lipid membranes the fluid-gel transition shows a clear trace in the change of the end-to-end distance. In our model we have measured the end-to-end distance of the lipids in the gel phase: $R_{e}^{g}=2.44[\Delta L]$ and in the liquid phase $R_{e}^{l}=2.525[\Delta L]$. The end-to-end distance in the vicinity of the protein is larger than in the fluid phase. From the plot (see fig 6.11) we can see that the lipids are stretched close to the inclusion's wall, they relax in correspondence of the first depletion peak of the density and they are slightly compressed in the depletion region.

\section{mean separation}

To compute the mean separation of the lipids we perform a Delaunay triangulation on the lipid position and calculate the radial area distribution of the triangles. A similar technique is used to study lipid packing Hömberg and Müller (2010)] or area fluctuation Shinoda and Okazi (1998)].

The Delaunay triangulation of a set of points (chain positions in this case) is a collection of edges where for each one we can find a circle containing the edge's vertices but no any other points. The radial profile of the mean separation is calculated obtaining the area of every triangle and assing the center of the triangle to the correspondent radial slab (see fig 6.12). In the graph (6.11) we see a clear decrease of the mean area close to the surface of the peptide where the lipids are more packed. In the depletion zone the mean separation between the lipids is larger showing a weakening of the membrane.
Figure 6.11: Comparison between the characteristic length of the following radial profiles of the chain conformations: mean separation, end-to-end distance, density, normal fluctuation. 
Figure 6.12: lhs) Delaunay triangulation on the lateral chain's position, blue and red lines distinguish between upper and lower monolayer. rhs) Sketch of a Delaunay triangulation around the peptide.
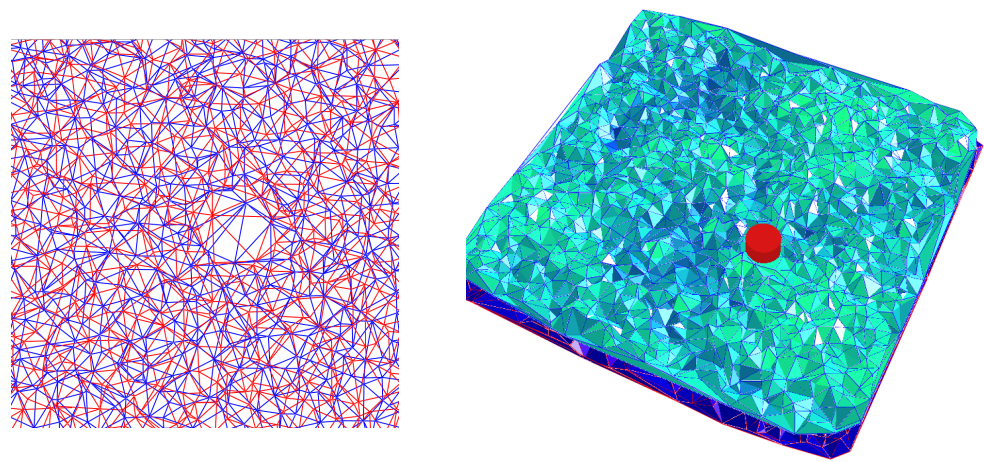

normal fluctuation

The normal fluctuation in the slab $s$ is the standard deviation of the normal position of the chains:

$$
\sigma_{z}(s):=\frac{\left\langle z_{c}^{2}(s)\right\rangle-\left\langle z_{c}(s)\right\rangle^{2}}{N_{c}(s)}
$$

The chains fluctuate closer to the wall of the membrane and less in the depletion zone.

\section{orientation angle fluctuation}

Similar to the previous calculation we show the fluctuation of the orientation angle of the chain:

$$
\sigma_{\alpha}(s):=\frac{\left\langle\alpha_{c}^{2}(s)\right\rangle-\left\langle\alpha_{c}(s)\right\rangle^{2}}{N_{c}(s)}
$$

The angular fluctuations are larger close to the peptide's wall, no other significant changes are visible.

\subsection{1 conclusion}

In this chapter we have used our coarse-grained solvent-free model to show how the presence of the transmembrane proteins locally modifies the continuum and discrete conformation of the membrane. The single effect of the protein superimpose for a particular disposition of transmembrane protein. We have calculated the interaction range between two proteins and quantified the identified the interpeptide distance where the superposition of the effects reaches its maximum. This distance was used to identify the ideal constellation of proteins to create a stable pore in a tensionless state.

The same results were obtained solving a continuum model parametrized from the analysis of the mechanical properties of a simulated membranes. The results of the continuum model have shown a perfect comparison with the results of the simulations. The numerical solution is many orders of magnitude faster than the simulations. This allowed us to fine explore the parameter space and calculate the interaction range for every protein description.

The stabilization of a pore is the break down of the continuum model, the membrane in this description is a continuum sheet and the pore formation is a discontinuity in the membrane. The pore stabilization could be parametrized setting a threshold value of the thickness corresponding to the point where the pore is stable in the simulations. We realize that the squared mesh used to describe the membrane is not flexible enough to describe the deformation around many peptides. A different mesh would be required around the peptide to represent the boundary conditions at the borders. The finite differences only work for a regular mesh, where every grid point is equally distanced. We suggest the extension of this calculation to finite element calculation to give a more accurate solution. 


\section{Chapter 7}

\section{line tension of the pore in presence of defectants}

Bilayer membranes are essential in isolating the cytosol from the extracellular fluid. The presence of a pore in membranes regulates communication between the inside and outside of the cell, enhances the fusion process between two opposed membranes Risselada et al. (2012)] and can cause the cell's death (apoptosis) Horton and Kelly (2009)]. Transient pores can spontaneously form and have been observed in giant unilamellar vesicles Karatekin et al. (2003a b)]. A pore can be otherwise stabilized by imposing a lateral pressure [Wang and Frenkel (2005)] or by transmembrane peptides Rzepiela et al. (2009)]. Peptide-stabilized pores are mainly distinguishable between barrel-stave and toroidal pores. Coarse-grained models are particularly well-suited to explore the size and shape evolution of pores and to estimate their line tension and activation energy [Litster (1975); Farago and Santangelo (2005); Wohlert et al. (2006)]. In this study we investigate the role of hydrophobic peptides in changing the shape and the activation barrier of a pore.

We observe that the effect of such proteins can be partially shielded by the addition of hydrophobic chains (oil). The oil represent the molecules of dodecan which is usually used as solvent in the preparation of synthetic membranes (pore spanned membrane Weiß and Enderlein (2012)]). The oil loses its configurational entropy and sits at the interface with the peptide relaxing the tension and improving the stability. In order to investigate lipid frustration inside the pore we add oil inside the membrane and observe the region where the oil partitions. We study and compare this effect in pores, proteins, and stalks.

\section{1 pore-protein interactions}

The discrete nature of the membrane (the lipids) allows the creation of temporary pores, facilitating the passage of compounds through the membrane Karatekin et al. (2003a b b ] . The energy barrier against pore formation is extremely high, due to the unfavourable interactions of the lipid tails with the solvent Wang and Frenkel (2005); Tolpekina et al. (2004); Ting et al. (2011)]. In order to create a stable pore, it is necessary to exert a lateral tension on the membrane or to shield the lipid tails from the contact with the polar solvent. The first case results in a toroidal pore (where the lipid head groups turn around the pore rim, see the rhs figure in fig 7.1 ), in the second case in a barrel stave pore (middle figure in fig 7.1, Illya and Deserno (2008)]).

Amphiphilic peptides (melittin Lin and Baumgaertner (2000); Yu et al. (2010); Yang et al. (2001)], magainin, hemagglutinin peptides [Risselada et al. (2012)] and bax- 
Figure 7.1: lhs) Snapshot showing the equilibrium position of the proteins around a pore. middle) Sketch of a barrel stave pore. rhs) Sketch of the superposition of the effect on the membrane thinning between a pore and a peptide.

FiguRE 7.2: Iterative process to determine the center, the position and the acylindricity of the pore. In the first snapshot we see a pore close to the inclusion, the pore is slightly asymmetric (lhs). We calculate the density for every grid point and we assign a threshold value. The red points are below the threshold, the green ones above (middle pictures). The center of the pore is the center of mass of the red points, the area is the area of the red pores and we construct a circle with that characteristics (middle right). The blue points represent the discretisation of the disk on the grid (rhs). derived peptides [García-Sáez et al. (2007)]) are short proteins where the polar residues face in the opposite direction with respect to the apolar ones. The polar face is in contact with the solvent and the apolar side is adsorbed to the hydrophobic moiety. We study the conditions of stability of such a pore depending on the lipid architecture.
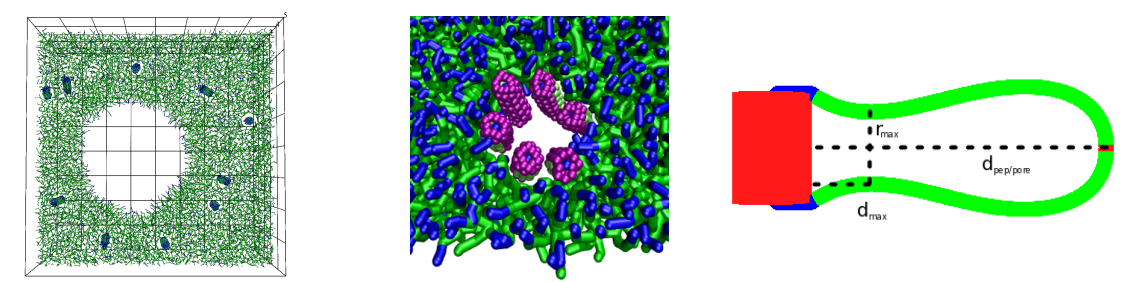

We have shown in the previous chapter that a particular disposition of transmembrane peptides can stabilize a toroidal pore. In this chapter we want to quantify the single contribution of a peptide on the pore shape and line tension. We create a stable pore by simulating a lipid membrane in the $N V T$ ensemble and we remove some lipids from the center of the system and we embed a transmembrane protein in the proximity of the pore rim. We study the mean protein/pore separation and pore acylindricity depending on the hydrophobic mismatch of the peptide.

Additionally, we calculate the changes in line tension of a pore in the presence of a peptide and compare the results with literature values García-Sáez et al. (2007)]. We notice that short hydrophobic chains (oil) added inside the membrane partition mainly in the region close to the peptide and the rim of the pore. As macroscopical effect the oil increases the line tension of the pore. To quantify the influence of the oil, we study the radial profiles of the bond length and lateral pressure around the defect.

\section{2 center, area and acylindricity of the pore}

The shape of the pore is approximately circular and we develop the following interactive method to find the radius of the pore and the deviation from the roundness.
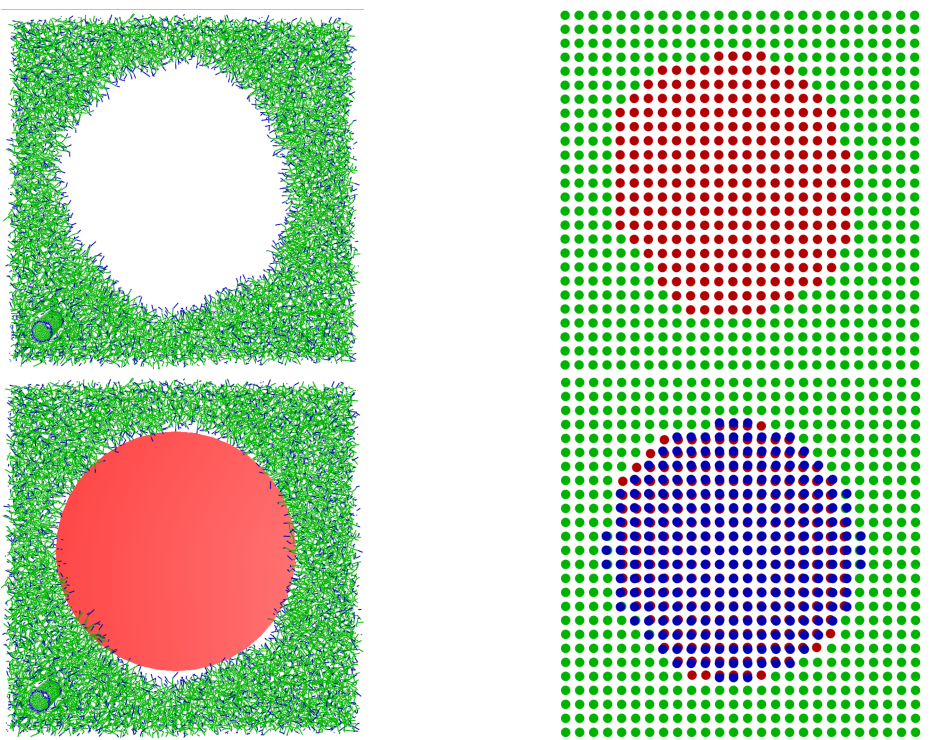

For every snapshot we:

- discretize the lateral dimensions in $N_{b i n}$ number of beads and calculate the bead density $\rho(i, j)$, 
- associate to every grid point a weight proportional to the inverse of the density, $w(i, j)=1 /(1+\rho(i, j))$,

- multiply each weight by the weights of the nearest neighbours,

$$
w^{\prime}(i, j)=w(i, j) \prod_{i^{\prime}=-1,1} w\left(i+i^{\prime}, j\right) \prod_{j^{\prime}=-1,1} w\left(i, j+j^{\prime}\right)
$$

- calculate the center of the pore, $\left(x_{\text {pore }}, y_{\text {pore }}, z_{\text {pore }}\right)$,

$$
x_{\text {pore }}=\frac{L_{x}}{N_{\text {bin }}} \frac{\sum_{i j} i w^{\prime}(i, j)}{\sum_{i j} w^{\prime}(i, j)} \quad y_{\text {pore }}=\frac{L_{y}}{N_{\text {bin }}} \frac{\sum_{i j} j w^{\prime}(i, j)}{\sum_{i j} w^{\prime}(i, j)} \quad z_{\text {pore }}=\frac{1}{N_{b} N_{c}} \sum_{i}^{N_{b} N_{c}} z_{i}
$$

$z_{i}$ is the normal coordinate of the particle $i$.

- we define a threshold, $\rho_{a}=1$, and an occupation function $o(i, j)$,

$$
o(i, j)=\left\{\begin{array}{lll}
1 & \text { if } \quad & \rho(i, j)>\rho_{a} \\
0 & \text { if } \quad & \rho(i, j)<\rho_{a}
\end{array}\right.
$$

- we calculate how many points are below the threshold $\left(N_{g}\right.$, green points in figure:7.2) and how many points are above the threshold $\left(N_{r}\right.$, red points in figure 7.2 ,

$$
N_{g}=\sum_{i j}(1-o(i, j)) \quad N_{r}=\sum_{i j} o(i, j)
$$

- we calculate the area, $A_{\text {pore }}$, and the radius of the pore, $r_{p o r e}$,

$$
A_{\text {pore }}=\frac{N_{r}}{N_{\text {bin }}^{2}} L_{x} L_{y} \quad r_{\text {pore }}=\sqrt{A_{\text {pore }} / \pi}
$$

- reweight each point with respect to the inverse of its distance from the former center of mass,

$$
w^{\prime \prime}(i, j)=w^{\prime}(i, j) /\left(\left(\frac{i L_{x}}{N_{b i n}}-x_{c m}\right)^{2}+\left(\frac{j L_{y}}{N_{b i n}}-y_{c m}\right)^{2}+0.01\right)
$$

- we recalculate the center of mass and iterate the last point until the value of the center of mass converges within $[0.5 \Delta L]$,

- the grid points inside a circle centered in $\left(x_{\text {pore }}, y_{\text {pore }}\right)$ and with radius $r_{\text {pore }}$ are described by the function:

$$
o^{\prime}(i, j)=\left\{\begin{array}{cc}
1 & \text { if }\left(\frac{i L_{x}}{N_{b i n}}-x_{c m}\right)^{2}+\left(\frac{j L_{y}}{N_{b i n}}-y_{c m}\right)^{2}<r_{\text {pore }}^{2} \\
0 & \text { otherwise }
\end{array}\right.
$$

- we calculate the acylindricity of the pore, $a_{\text {pore }}$,

$$
a_{\text {pore }}=\frac{\sum_{i j} o^{\prime}(i, j) o(i, j)+\sum_{i j}\left(1-o^{\prime}(i, j)\right)(1-o(i, j))}{\sum_{i j} o^{\prime}(i, j)}
$$

Expressed in words: the acylindricity is defined as the sum of points below the threshold inside the circle and of points above the threshold outside the circle divided by the total number of points inside the circle.

The determination of the center and the radius of the pore are essential to calculate the following radial profiles. 
FiguRE 7.3: Thickness profile between the peptide and the pore, rhs) characteristic quantities of the thickness profile between the pore and the peptide. $\lambda$ is the line tension of the pore, $r_{\text {pore }}$ is the pore radius and $P^{l}$ the lateral pressure.

$L_{x}=L_{y}=: L$ lateral simulation box size.

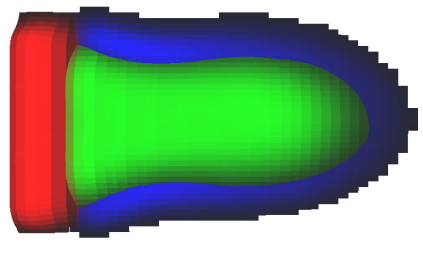

\begin{tabular}{cccc}
$h_{p}$ & $r_{\min }$ & $d_{\max }$ & $d_{\text {pep } / \text { pore }}$ \\
\hline 3.5 & 2.82 & 3.76 & 7.69 \\
4.0 & 5.29 & 0.00 & 5.29 \\
4.5 & 4.77 & 3.99 & 6.19 \\
5.0 & 6.78 & 3.43 & 7.33 \\
5.5 & 5.31 & 4.16 & 7.03 \\
6.0 & 5.65 & 0.00 & 5.65 \\
\hline$[\Delta L]$ & {$[\Delta L]$} & {$[\Delta L]$} & {$[\Delta L]$}
\end{tabular}

\section{3 thickness profile between nanoparticle and pore}

We have run $N V T$ simulations of planar membranes with the presence of a toroidal pore and a transmembrane protein described by different hydrophobic mismatch.

We analyze the thickness profile in a slab connecting the center of the pore with the center of the peptide as broad as the diameter of the peptide. From the thickness profile we extract the position of the minimum, $r_{\text {min }}$, the maximum thinning $d_{\text {max }}$, and the distance between the peptide and the pore, $d_{\text {pep/pore }}$. For each type of peptide we calculate the ternary $\left(r_{\min }, d_{\max }, d_{\text {pep/pore }}\right)$. For the simulation without oil the correspondent ternary is: $(6.08,3.36,10.32)[\Delta L]$, for simulation with oil it is : $(5.90,3.79,10.66)[\Delta L]$. This means that the presence of the oil pulls away the peptide from the rim of the pore.

From the table 7.3 we notice that the peptide is closer to the pore rim for $h_{p e p}=$ $4[\Delta L]$, i.e. no hydrophobic mismatch. By increasing the height of the peptide the separation from the pore rim increases until a turning point where the thickness profile does not show any minimum.

We have not observed any substantial modification of the pore radius and acylindricity using peptides with different hydrophobic mismatch.

\section{4 line tension of the pore}

To quantify the effect of peptides and oil on the stability of the membrane under lateral pressure, we calculate the line tension of the pore in the presence of these defectants. The systems we study are: (-,-) unperturbed membrane, (pep,-) one peptide, (-,oil) addition of oil chains, (pep,oil) addition of oil chains and one peptide. The number of oil chains is one tenth of the total number of lipid chains: $N_{\text {oil }}=N_{c} / 10$.

According to the classical nucleation theory [Litster (1975); Tolpekina et al. (2004)] the activation energy for the creation of a pore is:

$$
G=G_{0}+2 \pi r_{\text {pore }} \lambda-\pi r_{\text {pore }}^{2} P^{l}
$$

To calculate the activation energy we have to estimate the line tension of the pore.

We consider a membrane with fixed lateral dimension and with a pore of radius $r_{\text {pore }}$. The free energy of the system is:

$$
F\left(L, r_{\text {pore }}\right)=\frac{k_{\text {compr }}}{2 L^{2}}\left(L^{2}-\pi r_{\text {pore }}^{2}-\bar{a} N_{c}\right)^{2}+2 \pi \lambda r_{\text {pore }}
$$

The minimum of the free energy with respect to change in the pore radius, $r_{p o r e}$, is given by:

$$
\frac{\partial F}{\partial r_{\text {pore }}}=0 \quad \frac{k_{\text {compr }}}{L^{2}}\left(L-\pi r_{\text {pore }}^{2}-\bar{a} N_{c}\right)=\pi \lambda r_{\text {pore }}
$$

While the derivative of the free energy with respect to change in the lateral size $L$ is given by:

$$
\frac{\partial F}{\partial L}=2 L \frac{k_{\text {compr }}}{L}\left(L^{2}-\pi r_{\text {pore }}^{2}-\bar{a} N_{c}\right)=2 L \frac{\lambda}{r_{\text {pore }}}
$$




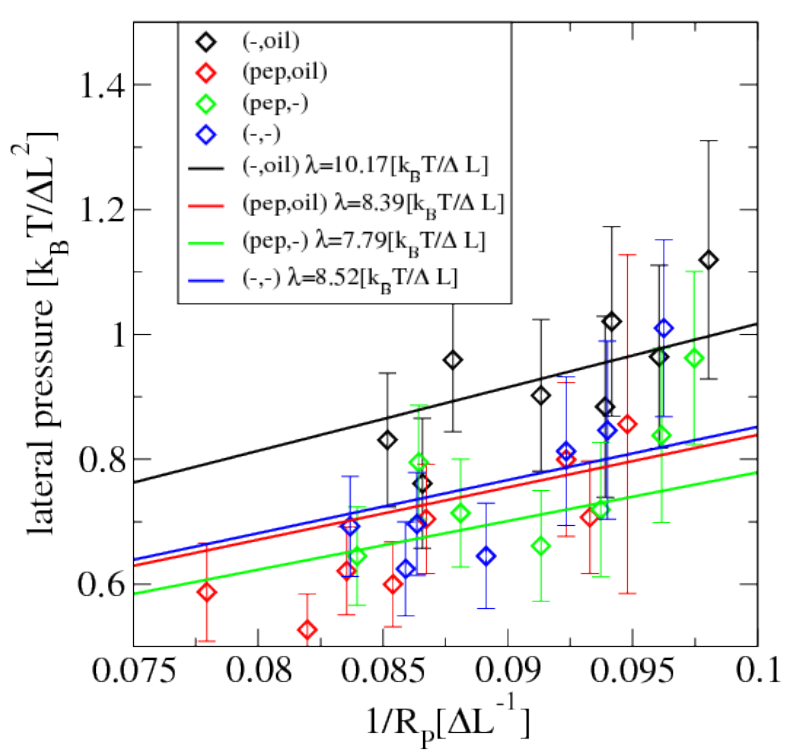

A change in the lateral dimension of the box, $L$, gives the following contribution:

$$
\frac{\partial F}{\partial L}=\frac{\partial}{\partial L}\left(L_{z} L^{2} P_{z z}^{l}\right)=2 L_{z} L P_{z z}^{l}
$$

Unifying (7.11) with 7.12 we finally obtain [Tolpekina et al. (2004)]:

$$
P_{z z}^{l}=\frac{\lambda}{r_{\text {pore }}}
$$

Starting from an already created pore we have increased slightly the lateral dimensions of the simulation box and calculated the radius of the pore once the system was reequilibrated.

We observe (see fig 7.4 that the presence of the peptide lowers the line tension of the pore while the oil increase it. The pore line tension is a well know quantity in literature, for a DOPC bilayer was calculated studying the pore closure dynamics after artificial poration of giant unilamellar vesicles $\lambda_{D O P C}=20.7 \pm 3.5[p N]$ [Karatekin et al. (2003a)] $\lambda_{D O P C}=27.7 \pm 2.5[p N]$ [Portet and Dimova (2010)]. In Karatekin et al. (2003a)] was additionally observed that the presence of cholesterol increases the line tension of the pore.

\section{5 partitioning of oil}

Since oil increases the energy barrier for the formation of the pore we want to study more in detail how the oil partition around the fusion objects and how its presence modifies the local arrangements of the lipids in its surroundings.

We observe from the density plots of the oil (see fig 7.5) a strong segregation of the oil at different interfaces. The oil can partition in the bilayer without changing the local hydrophobic density and relaxes the frustration of the lipid tails (due to stretching, compression, and tails splay). The partition has an energetic cost in terms of configurational entropy and gives us important information about where most of the frustration is released.

Around the peptide the oil sits close to the surface where the thickness of the membrane is largely above the bulk thickness. In this region the lipids are particularly
FiguRE 7.4: lhs) Lateral pressure as a function of the inverse of the pore size. The slope of the fitting curve is the line tension of the pore. rhs) Contour plot of the density of oil chains between the peptide and the pore.

$P_{z z}^{l}$ is the lateral tension of the system,

$P_{z z}^{l}=P_{z z}-\left(P_{x x}+P_{y y}\right) / 2 . L_{z}$ is the box size in the normal direction. 
Figure 7.5: Contour plots of the density of oil chains around a protein (lhs, lowest row), around a pore (middle), and around a stalk (rhs). The density is expressed in units of $\left[\Delta L^{-3}\right]$ for the solvent-free model, in $\left[\mathrm{nm}^{-3}\right]$ for the Martini simulations. In the plots are used different color mappings. In every system we add $N_{c} / 10$ of oil chains. The plots on the top row show the oil distribution inside the membrane and the plots on the middle row the point-by-point difference in the hydrophilic density between the system with and without the oil. The lines represent the isoline corresponding to half of the bulk density of the hydrophilic (green line) or the hydrophobic (blue line) beads. The lowest row shows the oil partitioning in Martini simulations (lhs) and the point-to-point difference in the hydrophobic density profile between the system without and with the oil.

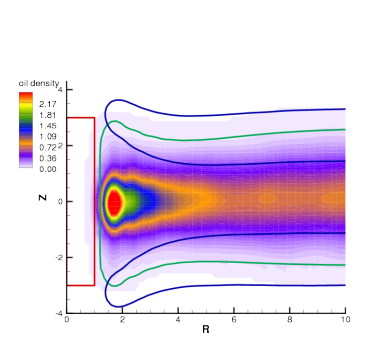

OF DEFECTANTS
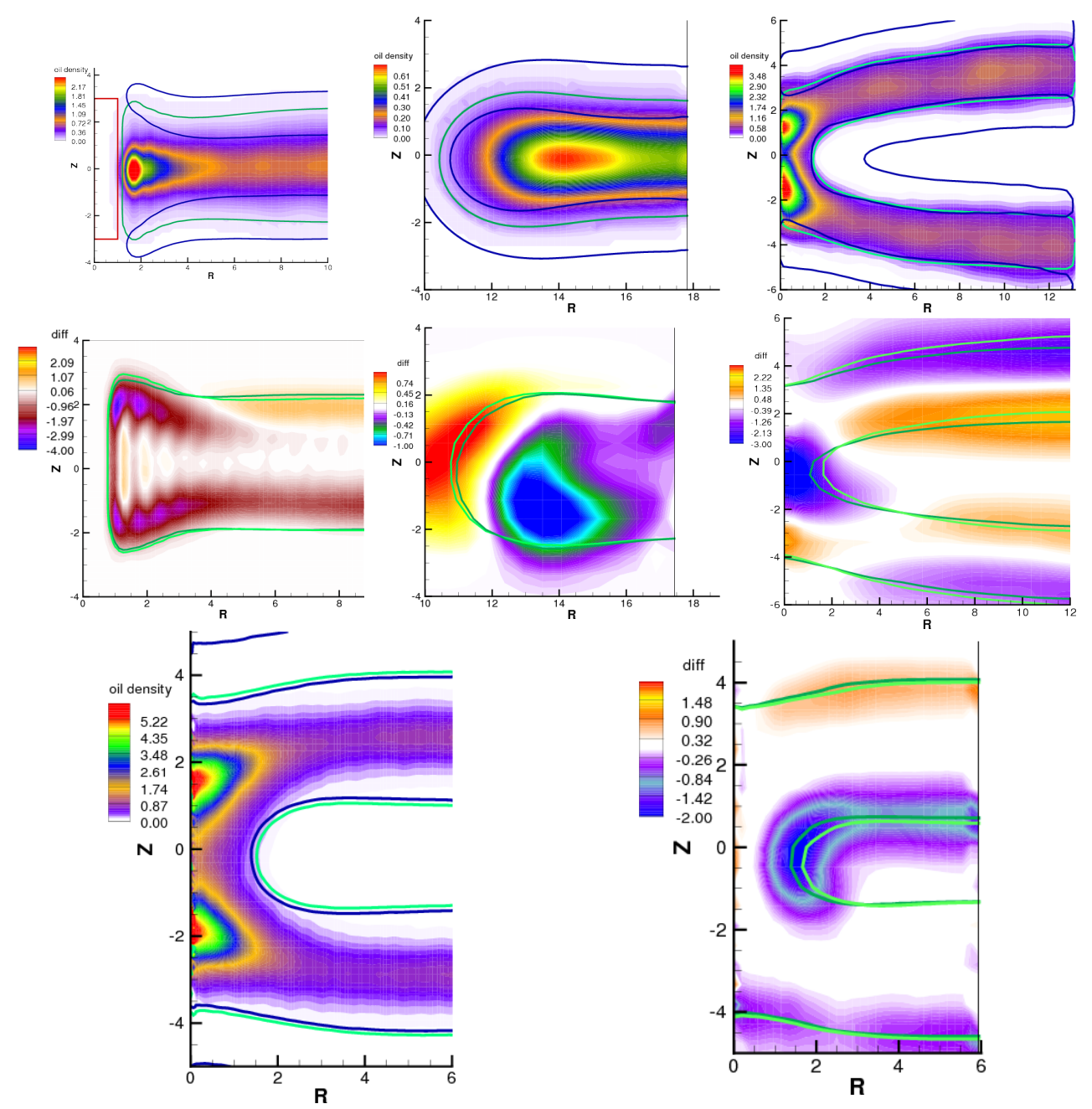

stretched and the presence of the oil reduces the extension of the chains. An interesting phenomenon is that the oil in the region where the lipids are depleted is depleted as well. The oil does not partition in the region of the overshoot of the thickness profile and does not modifies it (on the bottom lhs of the fig 7.5).

Looking at the oil partition inside the pore (middle column of the graph 7.5 ) we realize that the oil does not sit close to the rim even though the effect of the partition is strong. Probably the large compression of lipids around the rim of the pore does not allow the addition of other apolar material and the oil rather relaxes the conformation of the lipids in the region where the thickness profile is above the bulk value.

Referring to the stalk (rhs column of the fig:7.5) we observe that most of the oil sits at the lower and upper end of the stalk and not in the trunk. The surprising effect is that the presence of the oil does not change the indentation of the stalk, $d_{i}$, but increases the trunk, $d_{t}$. The indentation has a large energy cost in terms of bending and the expansion of the trunk should for the same reason increase the energy cost. Indeed we observe that the oil moves the membrane from the lamellar towards the inverted hexagonal phase causing the stalk to expand.

\section{6 bond length}

Since the density of the system around the defectants does not significantly change we would expect that the relaxation induced by the presence of the oil would not affect the total energy from the non-bonded interactions. In the solvent-free model the beads 
in the chains are connected by harmonic springs and the energetic contribution due to this interaction is straightforward to calculate. We have hence decided to calculate the distribution of hydrophobic bond length around the defectants.
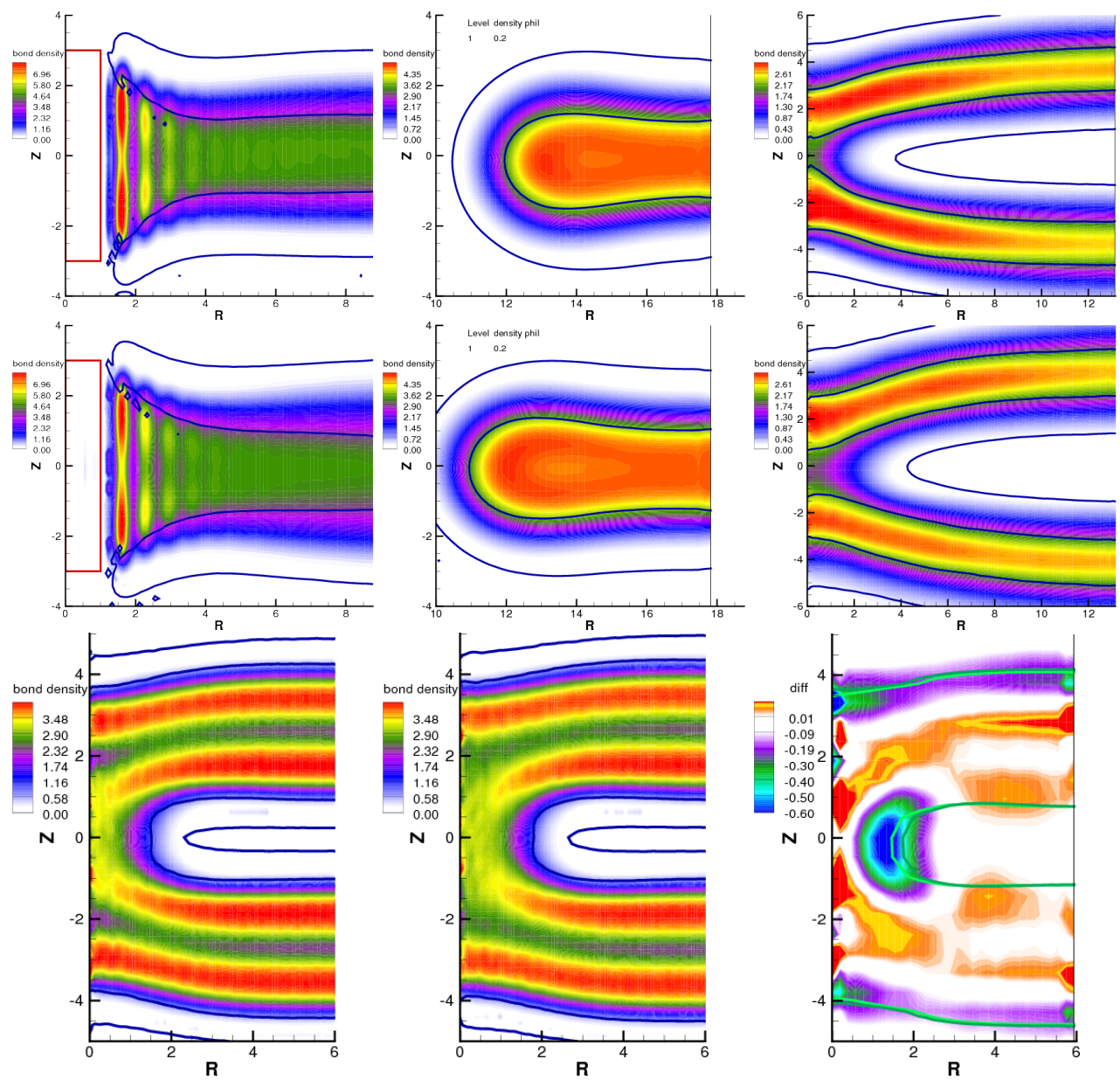

The hydrophobic bond length is simply the squared distance between two linked beads of the same type on the same chain

$$
r_{\text {bond }}^{2}=\left(x_{i}-x_{i+1}\right)^{2}+\left(y_{i}-y_{i+1}\right)^{2}+\left(z_{i}-z_{i+1}\right)^{2}
$$

where the monomer $i$ and the monomer $i+1$ are on the same type and belong to the same chain. We have considered both hydrophobic tails and oil chains and normalized the profiles by the volume of the slab they belong.

Looking at the plots of the bond distribution (see fig 7.6 ) we obtain important information about the stretching energy of the chains around the defectants but surprisingly we notice that the influence of the oil is really small. In the graph of the point-to-point difference between the bond length in the Martini simulations (third row and third column in figure 7.6 we see that most of the difference is concentrated in the trunk of the stalk. This is mainly due to the elongation of the stalk by the presence of oil but we do not register a change in the bond length by the presence of oil. The interesting difference between the Martini and the solvent free simulations is that most of the elongation is concentrated on the polar/apolar interface in the first case and in the equatorial plane in the second case. The difference is mainly connected with the parametrization of the Martini model into amphiphilic chains (see fig 5.2 and false the comparison between the two models (the interactions between the phosphate groups and the chains beads are different).
FiguRe 7.6: Contour plots of the density of bond length around a stalk. The density is expressed in units of $\left[\Delta L^{-2}\right]$ for the solvent-free simulations, $\left[\mathrm{nm}^{-2}\right]$ for the Martini. In the plots are used different definitions of the color mapping. The first row shows the contour plot of to three system without oil: around the peptide (lhs), around the pore (middle) and the stalk (rhs). The second row refers to the same three systems with the addition of oil in proportion of $1 / 10 N_{c}$. The lower row represents the contour plot of Martini simulations around a stalk: without oil (lhs), with oil (middle). The rhs graph of the lower line represents the point to point difference between the two graphs at its lhs. 


\section{7 lateral pressure profile}

We quantify the relaxation induced by the partitioning of the oil by calculating the differences between the lateral stress profiles.

Recalling the equation (1.8) the local pressure is the sum of the local densities (ideal gas contribution) and the virial (interaction term):

$$
P_{\alpha \beta}(\mathbf{x})=\rho(\mathbf{x})-\frac{1}{3 V} \sum_{i j}^{(\mathbf{x})} \frac{\mathrm{d} U_{i j}}{\mathrm{~d} r} \frac{x_{i j}^{\alpha} x_{i j}^{\beta}}{r_{i j}} \quad P_{\alpha \beta}^{t o t}=\frac{1}{V} \int \mathrm{d} \boldsymbol{x} P_{\alpha \beta}(\mathbf{x})
$$

The pressure profile is calculated on a fine grid along the radial and normal components. We assign to each grid point the densities of the hydrophobic and hydrophilic beads and the nine components of the virial tensor. To correctly assign the contributions of the interparticle interactions to each grid point, we draw a line connecting particle $i$ with particle $j$. The line is divided into 100 segments and for each segment we add $1 / 100$ of the value of the virial to the correspondent grid point. For each grid point we assign the density of the hydrophobic and hydrophilic beads and the nine components of the virial tensor.

$r_{s}$ is the distance in spherical coordinates: $r_{s}^{2}=x^{2}+y^{2}+z^{2}=$ $R^{2} x^{\prime 2}+R^{2} y^{\prime 2}+z^{2}$ $Z$ is the partition function of the system
We calculate the free-energy modification under a change in the radial distance, $R$ :

$$
\frac{\partial F}{\partial R}=-\frac{\partial}{\partial R} \ln Z \quad Z:=\int \mathrm{d} r_{s}^{N} e^{-H\left(r_{s}^{N}\right)}
$$

which corresponds to the energy of extending the pore by a quantity $\partial R$.

We can rescale the system units in normalized cylindrical coordinates:

$$
x=R x^{\prime} \quad y=R y^{\prime} \quad \mathrm{d} r_{s}=\mathrm{d} x \mathrm{~d} y \mathrm{~d} z=R^{2} \mathrm{~d} x^{\prime} \mathrm{d} y^{\prime} \mathrm{d} z^{\prime}=R^{2} \mathrm{~d} r_{s}^{\prime}
$$

We perform the derivative of the eq: (7.17):

$\frac{\partial F}{\partial R}=-\frac{\partial}{\partial R} \ln Z=-\frac{\partial}{\partial R} \ln \int \mathrm{d}\left(r_{s}^{\prime} R\right)^{N} e^{-H\left(r^{N}\right)}=-\frac{\partial}{\partial R}\left(\ln R^{2 N}+\ln \int \mathrm{d} r_{s}^{\prime^{N}} e^{-H\left(r_{s}{ }^{N}\right)}\right)$

We perform the derivative of the logarithm and we exchange the derivative with the $\partial_{R} \ln f(R)=f^{\prime}(R) / f(R)$, integral:

$\partial_{R} \int \mathrm{d} r_{s}^{\prime}=\int \mathrm{d} r_{s}^{\prime} \partial_{R}$

$$
\frac{\partial F}{\partial R}=-\frac{2 N}{R}+\frac{1}{Z} \int \mathrm{d} r_{s}^{\prime N} \frac{\partial H\left(r_{s}^{N}\right)}{\partial R} e^{-H\left(r_{s}^{N}\right)}=-\frac{2 N}{R}+\left\langle\frac{\partial H\left(r_{s}^{N}\right)}{\partial R}\right\rangle
$$
$\frac{\partial r_{s}}{\partial R}=\frac{\partial \sqrt{R^{2} x^{\prime 2}+R^{2} y^{\prime 2}+z^{2}}}{\partial R \quad \frac{x^{2}+y^{2}}{R r_{s}}}=$

The first term on the right hand side corresponds to the ideal gas contribution, the second term to the ensemble average of the virial. We perform the derivative of the distance $r$ with respect to the scaling distance $R$ :

$$
\frac{\partial F}{\partial R}=-\frac{2 N}{R}+\left\langle\frac{\partial r_{s}}{\partial R} \frac{\partial H\left(r_{s}^{N}\right)}{\partial r_{s}}\right\rangle=-\frac{2 N}{R}+\frac{1}{R}\left\langle\frac{x^{2}+y^{2}}{r_{s}} \frac{\partial H\left(r_{s}^{N}\right)}{\partial r_{s}}\right\rangle
$$

$V=\pi R^{2} L_{z} \quad$ We can hence connect the (eq 7.17) in terms of pressure in Cartesian coordinates:

$$
\frac{\partial F}{\partial R}=-\pi R L_{z}\left(P_{x x}^{t o t}+P_{y y}^{t o t}\right)=-\frac{1}{\pi} \int_{0}^{R} \mathrm{~d} r \int_{0}^{L_{z}} \mathrm{~d} z\left(P_{x x}(r, z)+P_{y y}(r, z)\right)
$$

From the previous equation (see eq 7.21) we can see that the transformation between cylindrical and Cartesian coordinates is:

$$
P_{r r}(r, z)=P_{x x}(r, z)+P_{y y}(r, z)
$$

For homogeneous systems the angular component of the pressure is identically zero Ollila et al. (2009)]. The lateral pressure is the difference between the direction we are looking at and the perpendicular directions:

$$
P_{z z}^{l}(r, z)=P_{z z}(r, z)-\left(P_{x x}(r, z)+P_{y y}(r, z)\right) / 2
$$



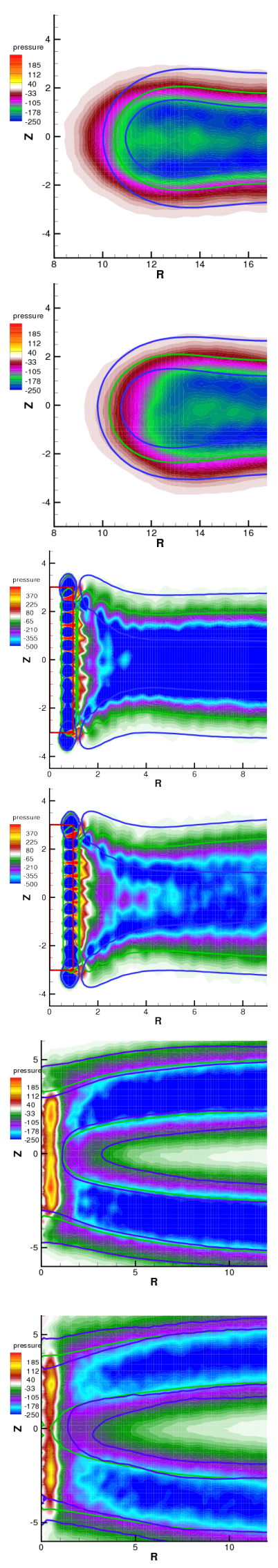
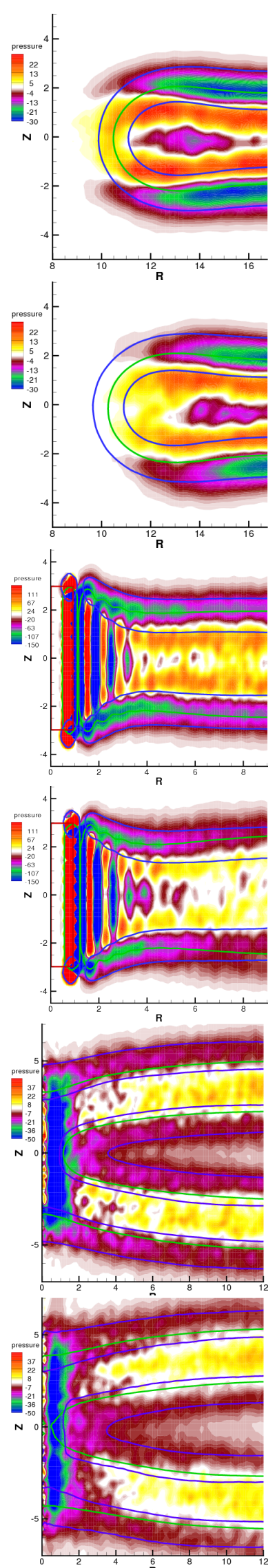

Figure 7.7: Lateral pressure profile around the peptide. On the lhs column is presented the radial component of the lateral pressure profile, $P_{r r}^{l}(r, z)$, on the rhs side column the normal component, $P_{z z}^{l}(r, z)$. In each column is used the same color map. The values of the pressure are expressed in units of $10^{-6}\left[k_{B} T / \Delta L^{3}\right]$. The radial profiles are calculated around: the pore (first row), the pore and oil (second row), the peptide (third row), the peptide and oil (forth row), the stalk (fifth row), the stalk and oil (sixth row). 
FiguRe 7.8: Lateral pressure profiles $\left(W_{z z}^{l}(z)\right)$, $\left.\left(W_{x x}^{l}(z)+W_{y y}^{l}(z)\right)\right)$ along the normal coordinates and density of hydrophobic and hydrophilic beads.

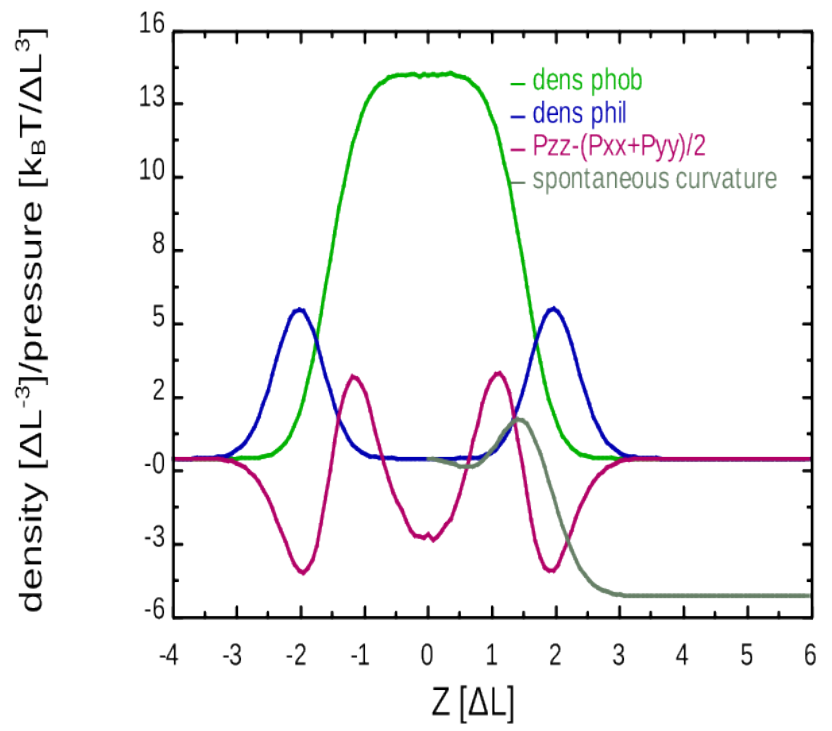

The lateral pressure profiles are particular useful to show where the interface between two medium sits and to calculate the surface tension between them.

The spontaneous curvature of the monolayer can be calculated from the pressure $c_{0}$ spontaneous curvature. profile Baoukina et al. (2010); Szleifer et al. (1992)]:

$$
k_{\text {ben }} c_{0}=\frac{1}{2} \int \mathrm{d} z|z| P_{z z}^{l}(z)
$$

The integration is performed from the center of the bilayer, $z=0$, and, using the value of table (4.3) for the bending rigidity, we obtain: $c_{0}^{m o n}=-0.36\left[\Delta L^{-1}\right]$.

In the plot (fig 7.7) we have shown the lateral pressure profiles (radial component on the lhs column and normal component on the rhs column) around the defectants. In the normal profiles we observe a clear interface between the hydrophobic/hydrophilic interface (as shown in the red line in fig 7.8 . . Around the peptide we can see a clear packing of the lipids that creates interfaces between the different lipid shells. In the center of the stalk we see another clear interface at the trunk of the stalk. In all the profiles we observe a slight influence in the profiles by the presence of oil. The effect is really small and affects the region where the oil density is larger and between the hydrophobic/hydrophilic interface.

\section{8 conclusion}

The presence of the peptide has a strong influence on the properties of a pore. In the previous chapter we have seen that the presence of the peptide weakens the membrane. In this chapter we have confirmed this prediction showing that the peptide lowers the line tension of the pore (and hence its activation energy) and we have calculated the mean separation between the peptide and the pore. The membrane deformations induced by a protein superimpose with the deformation induced by the pore and the protein sits at a fixed distance from the center of the pore. This distance depends on the hydrophobic mismatch of the peptide.

The oil has a larger conformation space than the lipids and can partition to release the frustration on the lipid chains. The effect of the oil is reflected in relaxing the pressure inside the bilayer. The presence of oil increases the pore line tension of the membrane and shields the weakening effect of the transmembrane protein. 


\section{Chapter 8}

\section{conclusions and outlook}

\section{1 summary}

In this work we have presented a soft solvent-free coarse-grained model to study the local modification of bilayer membranes around fusion objects: transmembrane proteins, pores and stalks.

The key features of the model is the Hamiltonian for the non-bonded interactions whose strength is controlled by the virial coefficients and the weighting functions. The virial coefficients are mainly set by the bulk compressibility, the incompatibility, and the coexistence density of the hydrophobic melt. We have shown as example, how the control of the bulk compressibility can reproduce the rich phase behaviour of lipid membranes and influence the material properties.

Thanks to the softness of the model we could calculate from thermal fluctuations some of these characteristic material properties: the volume and area compressibility, the elastic coupling between the two leaflets, and the bending rigidity. These properties were calculated by studying the fluctuations of the density, the area per chain, the membrane thickness and membrane height. To calculate the coupling moduli of this energetic contributions we have discretized the space and calculated the fluctuation of each quantity at the respective grid point. To determinate the correspondent coupling moduli we extrapolate the quantities fluctuations to infinite size dimensions.

The determination of these coupling moduli allowed us to construct a continuum model where the different deformations of the two coupled monolayers are represented by their respective free-energy contributions. In this model the surface curvature is controlled by the bending modulus, $k_{\text {ben }}$, the stretching by the surface tension, $\gamma^{\prime}$, and the height differences by the elastic coupling $k_{e l}$. The description of the model is based on differential geometry that we solve using discretized differential operators on grid points.

The continuum model is around 10000 times faster than the simulations and we use this model to finely explore the membrane modification by the presence of transmembrane proteins. The protein, due to its hydrophobic mismatch, modifies the conformation of the membrane and locally increases the distance between the two leaflets inducing local curvature and frustration to the lipids. With the combined use of molecular dynamics simulations and numerical calculation we explore the change in the membrane deformations depending on the hydrophobic mismatch of the protein and on the contact angle between the protein and the surrounding membrane.

The cumulative effect of the presence of many proteins amplifies the effect of the single protein within a certain range that we determine. Within this range we have observed that a particular arrangement of proteins stabilizes a pore in a tensionless state of the soft and coarse-grained model and shows the break-down of the continuum model.

We have looked at the discrete structure of the bilayer and investigated the change 
in conformation of the lipids and identified a region of weakening of the membrane. We have concluded that the presence of the peptide lowers the nucleation barrier of the pore and, for quantifying it, we have calculated the line tension of the pore and quantified the contribution of the peptide.

We have observed that the presence of oil partially shields the effect of the protein and increases the line tension. To understand the role of oil we study how it partitions around the peptide and how the oil relaxes the lateral tension in the proximity of the protein.

We have extended the same analysis to the local properties of the stalk. Using a novel method to determine the position of the stalk, we could calculate the density, thickness, and pressure profiles calculated in the case of the protein. We have observed that the properties of the stalk depend on the hydration between the two bilayers and we have compared the typical size of the stalk in different models and experiments and estimated the dependence of the bilayer repulsion on the intermembrane hydration and the lateral tension.

Finally we have developed a calculation method to determine whether an hydrophobic moiety is energetically more favorable inside the bilayer or in the solvent with a polymeric coating.

\section{2 conclusions}

In this work we have shown how to describe the complex physics of lipid membranes on large scale by using the three important features of our model: coarse-grained, soft and solvent-free.

\section{coarse-grained}

The physics of membrane has interesting properties in terms of macroscopic and microscopic modifications that coarse-grained models can exhaustively capture. We have shown as from the particle-based description we could refer to microscopic quantities and the relationships between the particle interactions and the macroscopic modifications. The simulations were used to build a continuum model that can capture the essential properties of big structures. Due to the discrete structure of the membrane topological modification are allowed starting from the local rearrangement of an handful of lipids. The time and length scales for the triggering of those processes are too small for experimental observations and the evolution of the shape modifications is too large for atomistic models. Using our coarse-grained model it was possible to describe complex topological changes like stalk and pore formation and fusion events. The fusion event is indeed a perfect application for coarse-grained models and we have observed and isolated fusion events trigger by the presence of an artificial pore or a stalk. Molecular dynamics simulations are particularly appropriate to construct specific system configurations and to study the interactions between fusion objects. The development of analysis tools to calculate local modifications of lipid chains around fusion objects was extensively used in this work to quantify characteristic length and energetic quantities of the lipid-mediated interactions between transmembrane proteins and pores. The effect of superimposition was always notable and has as most relevant feature the determination of a particular equilibrium distance and interaction range between different proteins and proteins and pores.

\section{soft}

A key aspect of this work was to show how via soft models we could calculate energetic contributions simply by averaging from thermal fluctuations. Molecular dynamics models that implement strong forces (hard core repulsion) show large fluctuations in energies and require more complicated sampling techniques to calculate, for example, 
the energetic coupling moduli of membrane deformation and bilayer repulsion. This allowed us to show the dependence of the bending modulus from the local curvature and to calculate and compare the line tension of the stalk and the pore.

\section{solvent-free}

Solvent-free models are particularly important to avoid the complex problem of equilibrating the number of water molecules under large membrane deformations. This characteristics allowed us to squeeze vesicles, to elongate stalks, and to sample intermembrane distances between two opposed membranes. Despite the lack of solvent molecules we could mimic the hydration between opposed bilayers by rescaling the head group interactions. This has as main consequence a change in the bilayer repulsion, a transition between the lamellar to the inverted hexagonal phase, and a condition for the stability of a stalk. The absence of solvent molecules is as well important to avoid artifacts during the fusion process caused by the incompressibility of water during the stalk expansion.

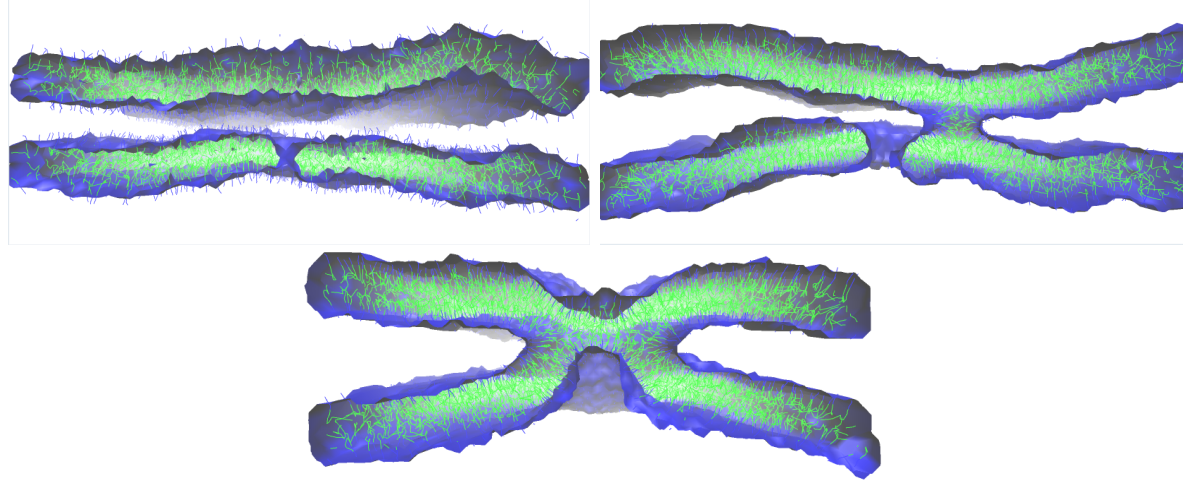

FiguRE 8.1: Cut through of the time evolution of the pore-triggered fusion pathway. An artificial pore is created in the lower membrane (lhs). A stalk is created close to the artificial pore (middle). The stalk elongates around the pore until it encloses it completely (rhs). The last configuration is called $\pi$ shaped hemifusion diaphragm and it is the last stage before the formation of a pore on the upper membrane and hence the fusion between the two membranes.

\section{soft, coarse-grained, solvent-free}

With this work we want therefore to point out the conceptual gain of using soft coarsegrained models. Despite the simplicity, these models can reproduce basic features of lipid bilayers even though the full experimental characteristics are not matched. These models allows the exploration of complex phenomena of lipid reordering with a clear description of the lipid conformations. By changing the model parameters and the boundary conditions we have induced important membrane shape deformations like the stabilization of a pore, the elongation of a stalk, and the fusion process. The superposition of the shape deformations influence as well the interactions between the fusion objects (pores, stalks, and peptides) and we have shown how the combined effects of these objects further modify the membrane structure (a pore stabilized by transmembrane proteins, a stalk elongates around a stable pore, a pore is formed in proximity of a stalk). 


\section{3 outlook}

Pore and stalk formation between two opposed membranes are competitive phenomena that depend on the lateral tension and dehydration between two bilayers. We have observed the fusion process between two opposed bilayers in a certain region of dehydration and lateral tension.

Alternatively we have observed that after the stabilization of a pore, a stalk is spontaneously formed, the stalk wonders close to the pore, it elongates circularly around it realizing a $\pi$ shaped hemifusion diaphragm (see fig8.1).

These fusion pathways point out a lipid-mediated interaction between stalks and pores that can be further investigated with the techniques developed in this work. The idea is to represent a pore by an external field or a transmembrane protein to be able to fix the position of the pore and control the line tension and the radial thickness profile. The stalk is free to move from the center of the pore and we can, by modifying the thickness profile of the pore, attract or repel the stalk. By modifying the line tension of the pore we could expand or retract the expansion of the stalk around the pore. This is intended to be a paradigm to describe the fusion process of the more accurate case of fusion driven by a barrel stave pore stabilized by a peptide bundle. 


\section{appendix}

\section{4 discrete solution of the continuum model}

To compute the Euler-Lagrange equation (see eq. 6.7) we have discretized the 2-d differential operator using finite differences. We take a regular grid on $N_{g}$ points per edge where every point is separated by a distance $h$. We take the following forth order differential operator:

$$
D=c_{4}^{\prime} \nabla^{4}+c_{3}^{\prime} \nabla^{3}+c_{2}^{\prime} \nabla^{2}+c_{1}^{\prime} \nabla+c_{0}
$$

We rescale the coefficients by

$$
c_{4}=\frac{c_{4}^{\prime}}{h^{4}} \quad c_{3}=\frac{c_{3}^{\prime}}{h^{3}} \quad c_{2}=\frac{c_{2}^{\prime}}{h^{2}} \quad c_{1}=\frac{c_{1}^{\prime}}{h}
$$

We define the following prefactors:

$$
\begin{aligned}
a_{-2} & =-\frac{c_{3}}{4}+\frac{3}{2} c_{4} \quad a_{-1}=-\frac{c_{1}}{2}+c_{2}+\frac{3}{2} c_{3}-6 c_{4} \quad a_{0}=c_{0}-2 c_{2}+9 c_{4} \\
a_{1} & =-\frac{c_{1}}{2}+c_{2}-\frac{3}{2} c_{3}-6 c_{4} \quad a_{2}=\frac{c_{3}}{4}+\frac{3}{2} c_{4}
\end{aligned}
$$

Its discretisation is:

$$
D=\left(\begin{array}{lllll}
a_{-2} & a_{-1} & a_{0} & a_{1} & a_{2}
\end{array}\right)
$$

We create an interaction matrix connecting the particle $(i, i)$ with the particle $(i+j, i)$ and with the particle $(i, i+j)$ by the weight $a_{j}$ where $j=-2,-1,0,1,2$. To each grid point is associated a thickness $d_{(i, i)}$. The interaction matrix is:

$$
m_{(i+j, i),(i, i)}=a_{j} d_{(i+j, i)} \quad m_{(i, i),(i, i+j)}=a_{j} d_{(i, i+j)} \quad j=-2,-1,0,1,2
$$

which is the convolution of the operator (eq 8.4) with the interaction matrix. We have to solve the following equation:

$$
M d=b
$$

The exact solution is given by inverting the matrix $M$.

$$
d=M^{-1} b
$$

The inversion of the matrix is done by a LU decomposition and the algorithm scales with $N_{g}^{4}$.

For system size larger than $N_{g}=60$ we use the Jacobi iterative method to speed up the solution. The method consists in separating the matrix as sum of the diagonal and non diagonal terms:

$$
M=M_{d}+M_{\bar{d}}
$$

The iterative solution is given by:

$$
d^{t}=M_{d}^{-1}\left(b-M_{\bar{d}} d^{t-1}\right) \quad d_{i, i}^{t}=\frac{1}{m_{(i, i)(i, i)}}\left(b_{i}-\sum_{j \neq i}^{N_{g}^{2}} m_{(i, i) j} d_{(i, i)}\right)
$$


In this case we can avoid to allocate any matrix and just compute:

$$
d_{(i, i)}^{t}=\frac{1}{d_{(i, i)}^{t-1}}\left(b_{(i, i)}-\sum_{-2<j<2} a_{j} d_{(i+j, i)}^{t-1}-\sum_{-2<j<2} a_{j} d_{(i, i+j)}^{t-1}+2 a_{0} d_{(i, i)}^{t-1}\right)
$$

Knowing the exact solution (by matrix inversion) we have observed a good convergence around 1000 time steps. The time gaining by the use of the iterative method is important already at $N_{g}>30$.

\section{5 interpolation/smoothing}

Most of the quantities calculated in this work were calculated discretisation the space and assigning a mean value to the correspondent grid point of the discretisation.

Figure 8.2: Different method of interpolating points in the space (from lhs to rhs): third-order splines, forth order finite differences, B-splines, polynomial.

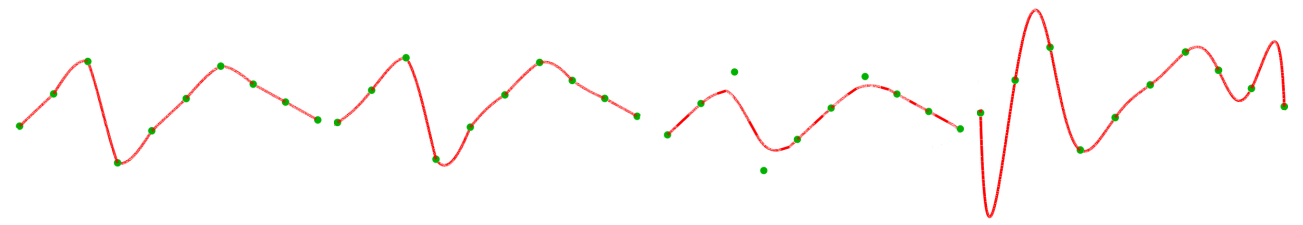

Each grid point is not independent from its neighbours especially in the motion of planar membranes or linear stalk. To induce correlation between neighbouring points we have used different interpolation and smoothing techniques that we are showing in this section.

An interpolating function is a continuum function $z_{i}(x)$ defined in the interval $x_{i-1}<$ $x<x_{i+1}$ by the neighbouring points $i+j$ where $j$ ranges between $-2<j<2$. The parabolic interpolating is defined by:

$$
\begin{aligned}
z_{i}(x) & =a_{2} x^{2}+a_{1} x+a_{0} \quad z_{i+1, i}=z_{i+1}-z_{i} \quad z_{i-1, i}=z_{i-1}-z_{i} \\
a_{2} & =\frac{z_{i+1, i} x_{i-1, i}-z_{i-1, i} x_{i+1, i}}{x_{i+1, i}^{2} x_{i-1, i}-x_{i+1, i} x_{i-1, i}^{2}} \quad a_{1}=\frac{z_{i-1, i}}{x_{i-1, i}}-a_{2} x_{i-1, i} \quad a_{0}=z(8
\end{aligned}
$$

Using the maximum likelihood:

$$
\begin{aligned}
z(x) & =a_{2} x^{2}+a_{1} x+a_{0} \\
X^{j} & :=\sum_{i}^{N} x_{i}^{j} \quad Y:=\sum_{i}^{N} y_{i} \quad X^{j} Y:=\sum_{i}^{N} x_{i}^{j} y_{i} \quad X^{j} \cdot Y:=\sum_{i}^{N} x_{i}^{j} \sum_{i}^{N} y_{i} \\
a_{2} & =\frac{\left(N X^{2} Y-X^{2} \cdot Y\right)\left(N X^{2}-X X\right)-\left(N X^{3}-X^{2} \cdot X\right)(N X Y-X \cdot Y)}{\left(N X^{4}-X^{2} \cdot x^{2}\right)\left(N X^{2}-X \cdot X\right)-\left(N X^{3}-X^{2} \cdot X\right)^{2}} \\
a_{1} & =\frac{(N X Y-X \cdot Y)-a_{2}\left(N X^{3}-X^{2} \cdot X\right)}{N X^{2}-X \cdot X} \\
a_{0} & =\frac{Y-a_{1} X-a_{2} X^{2}}{N}
\end{aligned}
$$

The third-order polynomial interpolation is:

$$
\begin{aligned}
z_{i}(x) & =a_{3} x^{3}+a_{2} x^{2}+a_{1} x+a_{0} \quad \text { where } \quad x_{i-1}<x<x_{i+1} \\
a_{3} & =\frac{\frac{z_{i+2, i}}{x_{i+2, i}}-\frac{z_{i-1,1}}{x_{i-1, i}}-\left(\frac{z_{i+1, i}}{x_{i+1, i}}-\frac{z_{i-1, i}}{x_{i-1, i}}\right)\left(x_{i+1, i}-x_{i-1, i}\right) / x_{i+2, i_{1}}}{x_{i+2, i}^{2}-x_{i-1, i}^{2}-\left(x_{i+1, i}^{2}-x_{i-i, 1}^{2}\right)\left(x_{i+2, i}^{2}-x_{i-1, i}^{2}\right) / x_{i+1, i-1}} \\
a_{2} & =\frac{z_{i+1, i} / x_{i+1, i}-z_{i-1} / x_{i-1, i}-a_{3}\left(x_{i+1, i}^{2}-x_{i-1, i}^{2}\right)}{x_{i+1, i-1}} \\
a_{1} & =z_{i-1, i} / x_{i-1, i}-a_{2} x_{i-1, i}-a_{3} x_{i-1, i}^{2} \quad a_{0}=z_{i}
\end{aligned}
$$


The 4 th-order spline on a regular mesh $\left(h=x_{i}-x_{i-1} \forall x_{i}\right)$ is:

$$
\begin{aligned}
z_{i}(x) & =a_{4} x^{4}+a_{3} x^{3}+a_{2}^{i} x^{2}+a_{1} x+a_{0}^{i} \quad \text { where } \quad x_{i-1}<x<x_{i+1} \\
a_{0} & =z_{i} \quad a_{2}^{i}=a_{2}^{i-1} \quad a_{2}^{i+1}=a_{2}^{\prime} \\
a_{4} & =3 a_{3}^{\prime} / h+\left(a_{2}^{i}-a_{2}^{\prime}\right) / h^{2} \quad a_{3}=a_{3}^{\prime}-6 a_{4} h \\
a_{1} & =z_{i+1, i} / h-a_{2} h-a_{3} h^{2}-a_{4} h^{3}
\end{aligned}
$$

where $a_{2}^{\prime}$ and $a_{3}^{\prime}$ are token from the cubic interpolation (see eq 8.13 and where the coefficient $a_{2}^{i}$ is calculated in the former cubic interpolation. The forth-order polynomial interpolation is:

$$
\begin{aligned}
z_{i}(x) & =a_{4} x^{4}+a_{3} x^{3}+a_{2} x^{2}+a_{1} x+a_{0} \quad \text { where } x_{i-1}<x<x_{i+1} \\
a_{4} & =\frac{z_{i-2} / 24-z_{i-1} / 6+z_{i} / 4-z_{i+1} / 6+z_{i+2} / 24}{h^{4}} \\
a_{3} & =\frac{z_{i-2} / 12+4 z_{i-1} / 6-4 z_{i+i} / 6+z_{i+2} / 12}{h^{3}} \\
a_{2} & =\frac{-z_{i-2} / 24+2 z_{i-1} / 3-5 / 4 z_{i}+2 z_{i+1} / 3-z_{i+2} / 24}{h^{2}} \\
a_{1} & =\frac{-z_{i-2} / 12+4 z_{i-1} / 6-4 z_{i+1} / 6+z_{i+2} / 12}{h} \quad a_{0}=z_{i}
\end{aligned}
$$

For the B-spline of the $o$ order:

$$
\begin{aligned}
z(x)=\sum_{i}^{N-o-2} z_{i} b_{o}\left(x-x_{i}\right) \quad b_{o}(x):=\frac{o+1}{o} \sum_{n}^{o} w n, o\left(x-x_{i}\right)_{+}^{o} \\
w_{n, o}:=\prod_{j=0, j \neq i}^{o+1} \frac{1}{x_{j}-x_{n}} \quad\left(x-x_{i}\right)_{+}^{o}:=\left\{\begin{array}{cc}
\left(x-x_{n}\right)^{o} & \text { if } x \geq x_{i} \\
0 & \text { if } \quad x<0
\end{array}\right.
\end{aligned}
$$

The extension of the interpolation functions in two dimensions is trivial on a regular grid.

The smoothing was executed convoluting a smoothing matrix, $S$, with the data matrix, $M$ :

$$
M^{\prime}=M \otimes S \quad M_{i, j}^{\prime}=\sum_{r=-N_{s} / 2}^{N_{s} / 2} \sum_{c=-N_{s} / 2}^{N_{s} / 2} M_{i+r, j+c} S_{r, s}
$$

The smoothing matrix has to fulfill the following properties:

$$
\sum_{r=-N_{s} / 2}^{N_{s} / 2} \sum_{c=-N_{s} / 2}^{N_{s} / 2} S_{r, s}=1
$$

The typical matrix used for the smoothing is the discretized Gaussian:

$$
S_{G}=\left(\begin{array}{ccccc}
e^{-\frac{2 x}{2 \sigma}} & e^{-\frac{\sqrt{3} x}{2 \sigma}} & e^{-\frac{\sqrt{2} x}{2 \sigma}} & e^{-\frac{\sqrt{3} x}{3 \sigma}} & e^{-\frac{2 x}{2 \sigma}} \\
e^{-\frac{\sqrt{3} x}{2 \sigma}} & e^{-\frac{\sqrt{2} x}{2 \sigma}} & e^{-\frac{x}{2 \sigma}} & e^{-\frac{\sqrt{2} x}{2 \sigma}} & e^{-\frac{\sqrt{3} x}{2 \sigma}} \\
e^{-\frac{\sqrt{2} x}{2 \sigma}} & e^{-\frac{x}{2 \sigma}} & 1 & e^{-\frac{x}{2 \sigma}} & e^{-\frac{\sqrt{2} x}{2 \sigma}} \\
e^{-\frac{\sqrt{3} x}{3 \sigma}} & e^{-\frac{\sqrt{2} x}{2 \sigma}} & e^{-\frac{x}{2 \sigma}} & e^{-\frac{\sqrt{2} x}{2 \sigma}} & e^{-\frac{\sqrt{3} x}{2 \sigma}} \\
e^{-\frac{2 x}{2 \sigma}} & e^{-\frac{\sqrt{3} x}{3 \sigma}} & e^{-\frac{\sqrt{2} x}{2 \sigma}} & e^{-\frac{\sqrt{3} x}{3 \sigma}} & e^{-\frac{2 x}{2 \sigma}}
\end{array}\right)
$$

This matrix is successively rescaled to 1 .

We use interpolation for the following calculations:

- estimate the energies of the bending and stretching of the mesh (polynomial), 
- reconstruct the shape of the linear stalk (B-spline),

- reconstruct the shape of the membrane's height and thickness (B-Spline),

- induce correlation in the radial profiles (Gaussian).

\section{6 domain decomposition}

For each step of a Monte Carlo simulation we have to calculate the distances between the probe particles and all the other particles within the cut-off distance. In the grand canonical ensemble the fluctuations of the number of particles is substantial and therefore we have to develop a fast and reliable scheme to calculate the interparticle distances. We have written a domain decomposition $\mathrm{c}++$ class to store all the information of the particle positions and to connect the particles by spatial neighbourhood.

usage

The class is meant to be used as a black box, the class is allocated at the beginning and disposes of a series of functions to fill and to clear the list and to operate on

CutOff2: squared cut-off distance,

Edge: box edges $\left(L_{x}, L_{y}, L_{z}\right)$. pNPart(): returns the total number of particles

DistRel: relative distance $(x, y, z, r)$ between the particles pointed by the iterators $i_{1}$ and $i_{2}$. the single particles (addition [AddPart], removal [RemPart], displacement [MovePart], and swapping [SwapPart]).

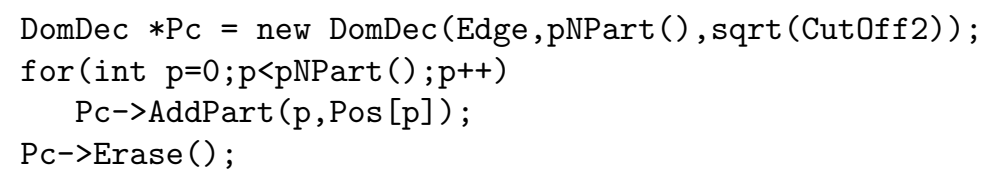

The class is particularly tuned for Monte Carlo simulations, for each time step a for loop calls the specific functions of the domain decomposition class. These functions consist in: an init function to assign all the private iterators, [SetCurr], a step function which increments the iterators [NextCurr], an if function that returns 0 when the iteration has reached the end [IfCurr]. Inside the for loop a function returns the squared interparticle distance between the two particles pointed by the iterators [Dist2Curr].

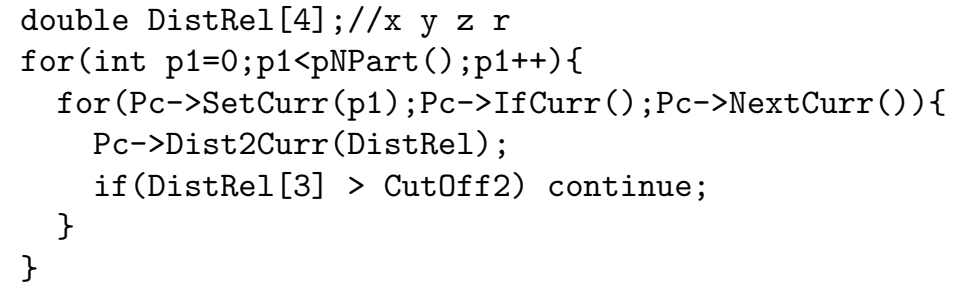

\section{structure}

To allocate the class we need to specify the cutoff distance of the system. The box volume is divided in small cells as wide as the cut-off distance we construct two structures, the cell and the particle structure. The cell structure contains the information of the first and last particle in the chain. The particle structure contains the position of the particle and two indices that point to the previous and the consecutive particle inside the cell. The consecutive particle of the last particle in the cell is marked as -1 and set the end of the list, the previous particle of the first particle in the cell is marked as -2 and set the beginning of the list. The iteration on the particle list can be done in both ways: from the last to the first or vice versa. This data structure is called linked cell list Frenkel (2002)]. 


\section{operations}

Every time that we select one particle $p_{1}$ we have to:

- identify the cell in which it is contained,

- set the first iterator on the particle $p_{2}$,

- set the second iterator on the first particle of the cell,

- obtain the list of the 26 neighbouring cells.

During the last operation an array with the 26 neighbouring cells, $c_{n}$, is allocated considering the periodic image convection. At the same time another array, $b_{i}$, is allocated with the information about periodic image convection in all the direction. This array corresponds to the particle $p_{1}$ and contains the number $-1,0$ or 1 for each direction. Once this array is allocated [Dist2Curr] returns the following squared interparticle distance:

$$
d^{2}\left(p_{1}, p_{2}\right)=\left(x_{p_{1}}-x_{p_{2}}+x_{b_{i}} L_{x}\right)^{2}+\left(y_{p_{1}}-y_{p_{2}}+y_{b_{i}} L_{y}\right)^{2}+\left(z_{p_{1}}-z_{p_{2}}+z_{b_{i}} L_{z}\right)^{2}
$$

This method avoids the computation of the minimum image convention for every interparticle distance improving the performance of the algorithm. The squared interparticle distance avoids to calculate the square root for interparticle distances within the squared cut-off radius and for many potentials that depends on even powers of the interparticle distances.

The iteration over the neighbouring particles is done in the following way:

- the second pointer, $i_{2}$, points to the particle $p_{2}$,

- if the particle $p_{2}$ is equal to the particle $p_{1}$ the iterator $i_{2}$ is incremented,

- if the iterator $i_{2}$ reaches the end of the cell, the iterator $i_{2}$ is assigned to the first particle of the next cell in the list $c_{n}$,

- if the iterator $i_{2}$ reaches the last particle of the last cell of the list $c_{n}$ the functions [IfCurr] interrupts the for loop.

This domain decomposition class is meant to be a separated entity of simple implementation and it robustness was explored during long Monte Carlo simulations. 



\section{Bibliography}

Adams, D. J. (1974). Chemical potential of hard-sphere fluids by monte carlo methods. Molec. Phys., 28, 1241.

Adams, D. J. (1975). Grand canonical ensemble monte carlo for a lennard-jones fluid grand canonical ensemble monte carlo for a lennard-jones fluid. Molec. Phys., 29, 307.

Adams, D. J. (1976). Calculating the low temperature vapour line by monte carlo. Molec. Phys., 32, 647.

Adams, D. J. (1979). Calculating the high-temperature vapour line by monte carlo. Molec. Phys., 37, 1241.

Aeffner, S., Reusch, T., Weinhausen, B., and Salditt, T. (2009). Membrane fusion intermediates and the effect of cholesterol: An in-house x-ray scattering study. The European Physical Journal E: Soft Matter and Biological Physics, 30, 205-214. 10.1140/epje/i2009-10466-x.

Allen, M. P. and Tildesley, D. J. (1991). Computer simulation of Liquids. Clarendon Press Oxford.

Apajalahti, T., Niemelä, P., Govindan, P. N., Miettinen, M. S., Salonen, E., Marrink, S. J., and Vattulainen, I. (2010a). Concerted diffusion of lipids in raft-like membranes. Faraday Discuss., 144, 411-30.

Apajalahti, T., Niemel, P., Govindan, P. N., Miettinen, M. S., Salonen, E., Marrink, S.J., and Vattulainen, I. (2010b). Concerted diffusion of lipids in raft-like membranes. Faraday Discuss., 144, 411-430.

Aranda-Espinoza, H., Berman, A., Dan, N., Pincus, P., and Safran, S. (1996). Interaction between inclusions embedded in membranes. Biophysical Journal, 71, 648-656.

Babuška, I. (1971). Error-bounds for finite element method. Numerische Matematik, 16, 322-333.

Baoukina, S., Marrink, S. J., and Tielemann, D. P. (2010). Lateral pressure profiles in lipid monolayers. Faraday discussions, 144, 393-409.

Bartels, C. (2000). Analyzing biased monte carlo and molecular dynamics simulations. Chemical Physics Letters, 331, 446-454.

Berendsen, H., Postma, J., van Gunsteren, W., DiNola, A., and Haak, J. (1984,). Molecular dynamics with coupling to an external bath. J. Chem. Phys., 81, 3684.

Berkowitz, M. L. and Raghavan, K. (1994). Interaction forces between membrane surfaces. Biomembrane Electrochemistry, 235, 3-25.

Bermudez, H., Braman, A. K., Hammer, D. A., Bates, F. S., and Discher (2002). Molecular weight dependence of polymersome membrane structure, elasticity, and stability. Macromolecules, 35, 8203. 
Bermudez, H., Hammer, D., and Discher (2004). Effect of bilayer thickness on membrane bending rigidity. Langmuir, 20, 540-543.

Blavatska, V. and Janke, W. (2010). Shape anisotropy of polymers in disordered environment. J. Chem. Phys., 133, 184903.

Brandt, E. G., Braun, A. R., Sachs, J. N., Nagle, J. F., and Edholm, O. (2011). Interpretation of fluctuation spectra in lipid bilayer simulations. Biophysical Journal, 100, 2104-2111.

Branningan, G. and Brown, F. L. H. (2006). A consistent model for thermal fluctuations and protein-induced deformations in lipid bilayers. Biophysical Journal, 90, $1501-1520$.

Branningan, G. and Brown, F. L. H. (2007). Contribution of gaussian curvature and nonconstant lipid volume to protein deformation of lipid bilayers. Biophysical Journal, 92, 864-876.

Cooke, I. R. and Deserno, M. (2005). Solvent-free model for self-assembling fluid bilayer membranes: Stabilization of the fluid phase based on broad attractive tail potentials. Journal of Chemical Physics, 123, 224710.

Cullis, P. R., Hope, M. J., and Tilcock, C. P. S. (1986). Lipid polymorphism and the roles of lipids in membranes. Chemistry and Physics of Lipids, 40, 127-144.

Daily, B. and Elson, E. (1984). Determination of the elastic area compressibility modulus of the erythrocyte membrane. Biophys Journal, 45, 671.

Daoulas, K. C. and Müller, M. (2010). Comparision of simulation of lipid membranes with membranes of block copolymers. Adv. Polym. Sci. "Polymer Membranes/Biomembranes", 224, 197-233.

Daoulas, K. C. and Müller, M. (2012). Exploring thermodynamic stability of the stalk fusion-intermediate with three-dimensional self-consistent field theory calculations. SoftMatter, -, submitted.

de Pablo, J. J., Laso, M., and Suter, U. W. (1992). Simulation of polyethylene above and below the melting point. J. Chem. Phys., 96, 2395-2403.

Discher, D. E., Boal, D. H., and Boey, S. K. (1998). Simulations of the erythrocyte cytoskeleton at large deformation. ii. micropipette aspiration. Biophys. J., 75, 1584-1597.

Dünweg, B. and Paul, W. (1991). Brownian dynamics simulation without gaussian number generator. Int. J. of Mod. Phy. C, 2(3), 817.

Espanol, P. and Warren, P. B. (1995). Dissipative particle dynamics: Bridging the gap between atomistic and mesoscopic simulation. Europhys. Lett., 30, 191.

Falck, E., Róg, T., Karttunen, M., and Vattulainen, I. (2008). Lateral diffusion in lipid membranes through collective flows. J. Am. Chem. Soc., 130, 44-45.

Farago, O. and Pincus, P. (2004). Statistical mchanics of bilayer membrane with a fixed projected area. J. Chem. Phys., 120, 2934-2950.

Farago, O. and Santangelo, D. (2005). Pore formation in fluctuating membrane. $J$. Chem. Phys., 122, 044901.

Fattal, D. R. and Ben-Shaul, A. (1993). A molecular model for lipid-protein interaction in membranes: the role of hydrophobic mismatch. Biophys. J., 65, 1795-809. 
Flory, P. J. (1941). Thermodynamics of high polymer solution. J. Chem. Phys., 9, 660 .

Fredrickson, G. H., Ganesan, V., and Drolet, F. (2002). Field-theoretic computer simulation methods for polymers and complex fluids. Macromolecules, 35, 16-39.

Frenkel, D. (2002). Understanding Molecular Simulation. Academic press, San Diego.

Frenkel, D., Mooij, G. C. A. M., and Smit, B. (1991). Novel scheme to study structural and thermal properties of continuously deformable molecules. J. Phys. Condens. Matter, 3, 30553-3076.

Fygenson, D. K. and Libchaber, J. F. M. A. (1997). Mechanics of microtubule-based membrane extension. Phys. Rev. Lett., 79, 4497-500.

Gaede, H. C. and Garwrisch, K. (2003). Lateral diffusion rates of lipid, water, and a hydrophobic drug in a multilamellar liposome. Biophysical Journal, 85, 1734-1740.

Gao, L., Shillcock, J., and Lipowsky, R. (2007). Improved dissipative particle dynamcis simulations of lipid bilayers. Journal of Chemical Physics, 126, 015101.

García-Sáez, A. J., Chiantia, S., Salgado, J., and Schwille, P. (2007). Pore formation by a bax-derived peptide: Effect on the line tension of the membrane probed by afm. Biophysical Journal, 93, 103-112.

Gonnella, G., Orlandini, E., and Yeomans, J. M. (1997). Spinodal decomposition to a lamellar phase: Effects of hydrodynamic flow. Phys. Rev. Lett., 78, 1695.

Goulian, M., Bruinsma, R., and Pincus, P. (1993). Long-range forces in heterogeneous fluid membranes. Europhys. Lett., 22, 145-150.

Gracià, R. S., Bezlyepkina, N., Knorr, R. L., Lipowsky, R., and Dimova, R. (2010). Effect of cholesterol on the rigidity of unsaturated membranes: fluctuation and electrodeformation analysis of giant vesicles. Soft Matter, 6, 1472-1482.

Grafmüller, A., Shillcock, J., and Lipowsky, R. (2009). The fusion of membranes and vesicles: Pathway and energy berriers from dissipative particle dynamics. Biophysical Journal, 96, 2658-2675.

Groot, R. D. and Warren, P. B. (1998). Dissipative particle dynamics: Bridging the gap between atomistic and mesoscopic simulation. J. Chem. Phys., 107(11), 4423.

Hamaker, H. C. (1937). The london-van der waals attraction between spherical particles. Physica IV, 10, 1058.

Harroun, T. A., Heller, W. T., Weiss, T. M., Yang, L., and Huang, H. W. (1999). Theoretical analisys of hydrophobic matching and membrane-mediated interactions in lipid bilayers containing gramicidin. Biophysical Journal, 76, 3176-3185.

Helfand, E. and Tagami, Y. (1971). Theory of the interface between immiscible polymer ii. J. Chem. Phys., 56(7), 3592.

Helfrich, P. and Jakobsson, E. (1990a). Calculation of deformation energies and conformations in lipid membranes containing gramicidin channels. Biophys. J., 57, $1075-84$.

Helfrich, P. and Jakobsson, E. (1990b). Calculation of deformation energies and conformations in lipid membranes containing gramicidin channels. Biophys. J., 57, $1075-1084$.

Helfrich, W. (1978). Steric interaction of fluid membranes in multilayer systems. $Z$. Naturforsch, 33a, 305. 
Helfrich, W. (1985). Effect of thermal undulations on the rigidity of fluid membranes and interfaces. J. Phys., 46, 1263-1268.

Hömberg, M. and Müller, M. (2010). Main phase transition in lipid bilayers: phase coexistence and line tension in a soft, solvent-free, coarse-grained model. J. Chem. Phys., 132, 155104.

Horton, K. L. and Kelly, S. O. (2009). Engineered apoptosis-inducing peptides with enhanced mitochondrial localization and potency. J. Med. Chem., 52, 3293-3299.

Huang, H. W. (1986a). Deformation free energy of bilayer membrane and its effect on gramicidin channel lifetime. Biophys. J., 50, 1061-1070.

Huang, H. W. (1986b). Deformation free energy of bilayer membrane and its effects on gramicidin channel lifetime. Biophys. J., 50, 1061-1070.

Illya, G. and Deserno, M. (2008). Coarse-grained simulations studies of peptideinduced pore formation. Biophysical Journal, 95, 4163-4174.

Israelachvili, J. N. (1998). Intermolecular and Surface Forces. Academic Press London.

Jahn, R. and Grubmüller, H. (2002). Membrane fusion. Current Opinion in Cell Biology, 14, 488-495.

Jarzynski, C. (1997). Nonequilibrium equality for free energy differences. Phys. Rev. Letter, $\mathbf{7 8}, 2690$.

Javanainen, M., Hammaren, H., Monticelli, L., Jeon, J.-H., Miettinen, M. S., MartinezSeara, H., Metzlera, R., and Vattulainen, I. (2013). Anomalous and normal diffusion of proteins and lipids in crowded lipid membranes. Faraday Discuss., -, --

Jungmann, N., Schmidt, M., Ebenhoch, J., Weis, J., and Maskos, M. (2003). Dye loading of amphiphilic poly(organosiloxane) nanoparticles. Angewandte Chemie International Edition, 42(15), 1714-1717.

Karatekin, E., Sandre, O., Guiouni, H., Morghi, N., Puech, P.-H., and BrochardWyart, F. (2003a). Cascade of transient pores in giant vesicle: Line tension and transport. Biophysical Journal, 84, 1734-1749.

Karatekin, E., Sandre, O., and Fran c. P.-W. (2003b). Transient pores in vesicles. Polymer International, 52, 486-493.

Katsov, K., Müller, M., and Schick, M. (2006). Field theoretic study of bilayer membrane fusion: Ii. mechanism of stalk-hole complex. Biophys. J., 90, 915.

Kim, K. S., Neu, J., and Oster, G. (1998). Curvature-mediated interactions between membrane proteins. Biophys. J., 75, 2274-2291.

Klauda, J. B., Brooks, B. R., and Pastor, R. W. (2006). Dynamical motions of lipids and a finite size effect in simulation of bilayers. Journal of Chemical Physics, 125, 144710 .

Kocun, M., Mueller, W., Maskos, M., Mey, I., Geil, B., Steinem, C., and Janshoff, A. (2010). Viscoelasticity of pore-spanning polymer membranes derived from giant polymersomes. Soft Matter, 6(11), 2508-2516.

Kocun, M., Lazzara, T., Steinem, C., and Janshoff, A. (2011). Preparation of solventfree, pore-spanning lipd bilayers: Modeling the low tension of plasma membranes. Langmuir, 27 (12), 7672-7680.

Koeleman, J. M. V. A. and Hoogerbrugge, P. J. (1993). An extended dissipative particle dynamics model. Europhys. Lett., 21, 363. 
Kozlov, M. M., Leikin, S., and Rand, R. P. (1994). Bending, hydration and institial energies quantitatively account for the hexagonal-lamellar-hexagonal reentrant phase transition in dioleoylphosphatidylethanolamine. Biophys. J., 67, 1603-1611.

Kozlovsky, J., Efrat, A., Siegel, D. A., and Kozlov, M. M. (2004). Stalk phase formation: Effects of dehydration and saddle splay modulus. Biophysical Journal, 87, $2508-2521$.

Kumar, S., Rosenberg, J. M., Bouzida, D., Swendsen, R. H., and Kollman, P. A. (1992). The weighted histogram analysis method for free-energy calculations on biomolecules. Journal of Computational Chemistry, 13, 1011-1021.

Kučerka, N., Nieh, M.-P., and Katsaras, J. (2011). Fluid phase lipid areas and bilayer thicknesses of commonly used phosphatidylcholines as a function of temperature. Biochimica et Biophysica Acta, 1808, 2761-2771.

Kyong, M. and Sheets, E. D. (2008). Vesicle diffusion close to a membrane: Intermembrane interactions measured with fluorescence correlation specroscopy. Biophysical Journal, 95, 5789-5797.

Leikin, S. and Parsegian, V. A. (1994). Temperature-induced complementarity as a mechanism for biomolecular assembly. Proteins, 19, 73-76.

Leikin, S., Parsegian, V., Rau, D., and Rand, R. (1993). Hydration forces. Annu. Rev. Phys. Chem., 44, 369.

Leikin, S., Rau, D. C., and Parsegian, V. A. (1995). Temperature-favored assembly of collagen is driven by hydrophilic rather than hydrophobic interactions. Nature Structural Biology, 2, 205-210.

Leikin, S. L., Rau., D. C., and Parsegian, V. A. (1994). Direct measurement of forces between self-assembled proteins: Temperature-dependent exponential forces between collagen triple helices. Proc. Natl. Acad. Sci., 91, 276-280.

Li, S., Eghiaian, F., Sieben, C., Herrmann, A., and Schaap, I. A. T. (2011). Bending and puncturing the inluenza lipid envelope. Biophysical Journal, 100, 637-645.

Lin, J.-H. and Baumgaertner (2000). Stability of a mellitin pore in a lipid bilayer: A molecular dynamics study. Biophysical Journal, 78, 1714-1724.

Litster, J. D. (1975). Stability of lipid bilayers and red blood cell membranes. Phys. Let., 53 A, 193-194.

Loison, C., Mareschal, M., Kremer, K., and Schimd, F. (2003). Thermal fluctuations in a lamellar phase of a binary amphiphile-solvent mixture: A molecular-dynamics study. Journal of Chemical Physics, 119, 13138.

Luckey, M. (2008). Membrane Structural Biology. Cambridge University Press: New York.

Mark, J. E. (2007). Physical Properties of Polymers Handbook. Springer Cincinnati Ohio.

Markvoort, A. J. and Marrink, S. J. (2011). Lipid acrobatics in the membrane fusion arena. Current Topics in Membranes, 68, 259-294.

Marrink, S. J. and Mark, A. E. (2003). The mechanism of vesicle fusion revealed by molecular dynamics simulations. J. Am. Chem. Soc., 125, 11144-11145.

Marrink, S. J., Risselada, H. J., Yefimov, S., Tieleman, D. P., and de Vries, A. H. (2007). The martini force field: Coarse grained model for biomolecular simulations. J. Phys. Chem., 111, 7812-7824. 
Marčelja, S. (1976). Lipid-mediated protein interaction in membranes. Biochim. $c$ Biophys. Acta, 455, 1-7.

Maskos, M. (2006). Influence of the solvent and the end groups on the morphology of cross-linked amphiphilic poly (1,2-butadiene)-b-poly(ethylene oxide) nanoparticles. Polymer, 47, 1172-1178.

May, S. (2002). Membrane perturbations induced by integral proteins: Role of comformational restrictions of the lipid chains. Langmuir, 18, 6356-6364.

McIntosh, T. J. and S.A.Simon (1994). Hydration and steric pressures between phospholipid bilayers. Annu. Rev. Biophys. Biomol.Struct., 23, 27-51.

Meyer, H., Wittmer, J. P., Kreer, T., Beckrich, P., Johner, A., Farago, J., and Baschnagel, J. (2008). Static rouse modes and related quantities: Corrections to chain ideality in polymer melts. Euro Phys. J. Lett., 26, 25-33.

Miao, L., Fourcade, B., Wortis, M. R. M., and Zia, R. K. P. (1991). Equilibrium budding and vesiculation in the curvature model of fluid lipid vesicles. Phys. Rev. A, 43, 6843-56.

Miao, L., Seifert, U., Wortis, M., and Dobereiner, H. (1994). Budding transitions of fluid-bilayer vesicles: the effect of area-difference elasticity. Phys. Rev. E, 49, $5389-407$.

Mooij, G. C. A. M. and Frenkel, D. (1994). The overlapping distribution method to compute chemical potentials of chain molecules. J. Phys.: Condens. Matter, 6, $3879-3888$.

Müller, M. (1999). Miscibility behavior and single chain properties in polymer blends: a bond fluctuation model study. Macromol. Theory Simul., 8, 343374.

Müller, M. and Schick, M. (1996). Structure and nucleation of pores in polymeric bilayers: A monte carlo simulation. J. Chem. Phys., 105, 8282.

Müller, M., Katsov, K., and Schick, M. (2006). Biological and synthetic membranes: What can be learned from a coarse-grained description? Phys. Rep, 434, 113-176.

Müller, M., Katsov, K., and Schick, M. (2003). A new mechanism of model membrane fusion determined from monte carlo simulation. Biophys J, 85, 1611-1623.

Müller, M., Smirnova, Y. G., Marelli, G., Fuhrmans, M., and Shi, A. C. (2012). Transition path from two apposed membranes to a stalk obtained by a combination of particle simulations and string method. Phys. Rev. Lett., 108, 228103.

Neder, J., West, B., Nielaba, P., and Schmid, F. (2010). Coarse-grained simulations of membrane under tension. Journal of Chemical Physics, 132, 115101.

Nielsen, C., Goulian, M., and Andersen, O. S. (1998). Energetics of inclusion-induced bilayer deformations. Biophys. J., 74, 1966-83.

Niemelä, P. S., Miettinen, M. S., Monticelli, L., Hammaren, H., Bjelkmar, P., Murtola, T., Lindahl, E., and Vattulainen, I. (2010). Membrane proteins diffuse as dynamics complexes with lipids. J. Am. Chem. Soc., 132, 7574-7575.

Norizoe, Y., Daoulas, K. C., and Müller, M. (2010). Measuring excess free energies of self-assembled membrane structures. Faraday Discussion: Multiscale Modelling of Soft Matter, 144, 363.

Ollila, O. H. S., Risselada, H. J., Louhivouri, M., Lindahl, E., Vattulainen, I., and Marrink, S. J. (2009). 3d pressure field in lipid membranes and membrane-protein complexes. Physical Review Letter, 102, 078101. 
Orlandini, E. (2008). Notes on the Lectures about Statistical Mechanics and Stochastic Processes. Università degli studi di Padova.

Pagonabarraga, I. and Frenkel, D. (2001). Dissipative particle dynamics for interacting systems. J. Chem. Phys, 115, 5015.

Partenskii, M. B. and Jordan, P. C. (2002). Membrane deformation and the elastic energy of insertion: perturbation of membrane elastic constants due to peptide insertion. J. Chem. Phys., 117, 10768-76.

Pastorino, C., Kree, T., Müller, M., and Binder, K. (2007). Comparison of dissipatice particle dynamics and langevin thermostats for out-of equilibrium simulations of polymeric system. Phys. Rev. E, 76(2), 026706.

Peliti, L. and Leibler (1985). Effects of thermal fluctuations on systems with small surface tension. Phys. Rev. Lett., 54, 1690-1693.

Plischke, M. and Bergensen, B. (1994). Equilibirium Statistical Physics. Cambdrigde University Press.

Portet, T. and Dimova, R. (2010). A new method for measuring edge tensions and stability of lipid bilayers: Effect of membrane composition. Biophysical Journal, 99, 3264-3273.

Prost, J. and Bruinsma, R. (1996). Shape fluctuations of active membranes. Europhys. Lett., 33, 321-326.

Rand, R. P. and Parsegian, V. A. (1989). Hydration forces between phospholipid bilayers. Biochim. Biophys. Acta, 988, 351-376.

Rand, R. P., Fuller, N., Parsegian, V. A., and Rau, D. C. (1988). Variation in hydration forces between neutral phospholipid bilayers: Evidence for hydration attraction. Biochemistry, 27, 7711-7722.

Reister, E. and Seifert, U. (2005). Lateral diffusion of a protein on a fluctuating membrane. Europhys. Lett., 71, 859-865.

Risselada, H., Marelli, G., Fuhrmans, M., Smirnova, Y., Grubmüller, H., Marrink, S., and Müller, M. (2012). Line tension controlled mechanism for influenza fusion. Plos One, 7, e38302.

Risselada, H. J. and Grubmüller, H. (2012). How snare molecules mediate membrane fusion: Recent insights from molecular simulations. Curr Opin Struc Biol, 22, 187-196.

Rudnick, J. and Gaspari, G. (1986). The aspherity of random walks. J. Phys. A: Math. Gen., 19, L191.

Rzepiela, A. J., Sengupta, D., Goga, N., and Marrink, S. J. (2009). Membrane poration by antimicrobial peptides combining atomistic and coarse-grained descriptions. Faraday Discussions, 144, 431-443.

Schick, M., Katsov, K., and Müller, M. (2005). The central role of line tension in the fusion of biological membranes. Mol Phys, 103, 3055.

Schmidt, U., Guigas, G., and Weiss, M. (2008). Cluster formation of transmembrane proteins due to hydrophobic mismatching. Physical Review Letter, 101, 128104.

Seifert, U. (1997). Configuration of fluid membranes and vesicles. Advanced in Physics, 46, 13-137. 
Seifert, U., Berndl, K., and Lipowsky, R. (1991). Shape transformations of vesicles: phase diagram for spontaneous-curvature and bilayer-coupling models. Phys. Rev. A, 44, 1182-202.

Shillcock, J. C. and Lipowsky, R. (2007). Visualiying soft matter: Mesoscopic simulations of membranes, vesicles and nanoparticles. Biophys. Rev. and Lett., 2, 33-55.

Shing, K. S. and Gubbins, K. E. (1981). The chemical potential in dense fluids and fluid mixtures via computer simulation. test particle method with umbrella sampling. Molecular Physics, 43, 7117-721.

Shing, K. S. and Gubbins, K. E. (1982). The chemical potential in dense fluids and fluid mixtures via computer simulation. Molecular Physics, 46, 1109-1128.

Shinoda, W. and Okazi, S. (1998). A voronoi analysis of lipid area fluctuation in a bilayer. Journal of Chemical Physics, 109, 1517.

Sintes, T. and Baumgärtner, A. (1997). Protein attraction in membranes induced by lipid fluctuatuions. Biophysical Journal, 73, 2251-2259.

Smirnova, Y. (2012). -. private communications, -, --

Smirnova, Y. G., Marrink, S.-J., Lipowsky, R., and Knecht, V. (2010). Solvent-exposed tails as prestalk transition states for membrane fusion at low hydration. J. Am. Chem. Soc., 132, 6710-6718.

Smit, B. (1995). Grand canonical monte carlo simulations of chains molecules: adsorption isotherms in zeolites. Molecular Physics, 85, 153-172.

Stiasny, K. and Heinz, F. X. (2004). Effect of membrane curvature-modifying lipids on membrane fusion by tick-borne encephalitis virus. J Virol., 16, 8536-42.

Szleifer, I., Kramer, D., Sen-Shaul, A., Gelbart, W. M., and Safran, S. A. (1990). Molecular theory of curvature elasticity in surfactants. J. Cjem. Phys., 92, 6800.

Szleifer, I., Kramer, D., Ben-Shaul, A., Gelbart, W. M., and Safran, S. A. (1992). Molecular theory of curvature elasticity in surfactant films. J. Chem. Phys., 92, 6800-6817.

Theodorou, D. N. and Suter, U. W. (1985). Shape of unperturbed linear polymers polypropylene. Macromolecules, 18, 1206.

Ting, C. L., Appelö, D., and Wang, Z.-G. (2011). Minimum energy path to membrane pore formation and rupture. Phys. Rev. Lett., 106, 168101.

Tolpekina, T. V., den Otter, W. K., and Briels, W. J. (2004). Simulations of stable pores in membranes: System size dependence and line tension. J. Chem. Phys., 121(16), 8014 .

Trominov, S. Y., Nies, E. L. F., and Michels, M. A. J. (2002). Thermodynamic consistency in dissipative particle dynamics simulations of strongly nonidel liquids and liquid mixtures. J. Chem. Phy., 117(20), 9383.

van der Spoel, D., Lindahl, E., Hess, B., Groenhof, G., Mark, A., and Berendsen, H. (2005). Gromacs: Fast, flexible, and free. J. Comp. Chem, 26, 1701.

Venturoli, M., Smit, B., and Sperotto, M. M. (2005). Simulation studies of proteininduced bilayer deformations, and lipid-induced protein tilting, on a mesoscopic model for lipid bilayers with embedded proteins. Biophysical Journal, 88, 17781798 . 
Venturoli, M., Sperotto, M. M., Kranenburg, M., and Smit, B. (2006). Mesoscopic models of biological membranes. Physics Reports, 437, 1-54.

Waheed, Q. and Edholm, O. (2009). Undulation contribution to the area compressibility in lipid bilayer simulations. Biophysical Journal, 97, 2754-2760.

Wang, Z.-J. and Frenkel, D. (2005). Pore nucleation in mechanically stretched bilayer membranes. The Journal of Chemical Physics, 123, 154701.

Weiß, K. and Enderlein, J. (2012). Lipid diffusion within black lipid membranes measuered with dual-focus fluorescence correlation spectroscopy. ChemPhysChem, 13, 990-1000.

West, B., Brown, F. L. H., and Schimdt, F. (2009). Membrane-protein interactions in a generic coarse-grained model for lipid bilayer. Biophysical Journal, 96, 101-115.

Widom, B. (1963). Some topics in the theory of fluids. J. Chem. Phys., 39, 2802.

W.Müller, Koynov, K., Fischer, K., Pierrat, S., Schärtl, W., Basche, T., and Maskos, M. (2008). Hydrophobic shell of pb-b-peo vescicles. Macromolecules, 42, 357-361.

Wohlert, J., den Otter, W. K., Edholm, O., and Briels, W. J. (2006). Free energy of a trans-membrane pore calculated from atomistic molecular dynamics simulations. The Journal of Chemical Physics, 124, 154905.

Wu, D., Fredrickson, G. H., Carton, J. P., Ajdari, A., and Leibler, L. (1995). Distribution of chain ends on a surface of polymer melt: Compensation effects and surface tension. Journal of Polymer science, 33, 2373-2389.

Yang, K. and Ma, Y.-Q. (2012). Computer simulations of fusion, fission and shape deformation in lipid membranes. Soft Matter, 8, 606-618.

Yang, L., Harroun, T. A., Weiss, T. M., and Huang, H. W. (2001). Barrel-stave model or toroidal model? a case study on melittin pores. Biophyisical Journal, 81, $1475-1485$.

Yu, Y., Vroman, J. A., Bae, S. C., and Granick, S. (2010). Vesicle budding induced by a pore-forming peptide. J. Am. Chem. Soc., 132, 195-201.

Zemel, A., Ben-Shaul, A., and May, S. (2008). Modulation of the spontaneous curvature and bending rigidity of lipid membranes by interfacially adsorbed amphipathic peptides. J. Phys. Chem., 112, 6988-6996. 


\section{acknowledgments}

The realisation of this work would not have been possible without the help of many people. First of all I want to thank my supervisor Prof. Dr. Marcus Müller for having offered me this chance. I've particularly profited of his scientific acumen and his large understandings of physics. I particularly remember the astonishment reading his e-mail during the Summer 2007 when he accepted me as master student in his group and when he confirmed me as a PhD student.

After that, my colleagues Kostas Daoulas and Martin Hömberg, were crucial during my discovery phase. I especially thank Kostas for his care and the rigour he was using to answer to my questions and Martin who has guided me in using the program and understand the model. His formality gave me an important hint to handle professional relationships and to learn cooperative work.

Successively, during my exploring phase, I remember at most the time spent discussing with Jelger Risselada as turning point of my PhD. Those discussions gave me a deep understanding on the physics of membranes and gave me the motivations and the ideas to continue my $\mathrm{PhD}$. The successive contributions of Gregory Bubnis enriched those discussions and started an interesting collaboration.

After this period I felt much more confident in discussing and understanding physics and in starting a collaborative phase. To start the collaborative phase was important to follow the meetings of my founding project, the Sondern Forschung Bereich under the project number SFB:803: "Funktionalität kontrolliert durch Organisation in und zwischen Membranen". From this meetings I've profited mostly discussing with Sebastian Äffner about stalk morphology and hydration, Gregory Bubnis about energy of bending, Wensi Gao about membrane fusion, Christopher Battle for line shape reconstruction, and Kerstin Weiß for lipid diffusion.

From my colleague was really important the input gave me from Yuliya Smirnova (most of the forth chapter), from Ulrich Welling (implementation of algorithms), Daniela Cadamuro (mathematical background), and from Yuki Norizoe, Veronica Chappa and Timo Fischer (Monte Carlo simulations). Strong support came as well from Fabién Leonforte, Andre Galuschko, Nikita Tretyakov, Murat Mülayim, Marc Fuhrmans, Israel Barragan Vidál, Anna Cavallo, and Claudio Pastorino.

Another really important source for discussions and collaborations came from my graduate school, the GGNB, and for that I thank the organisation and especially the particular care of Antje Erdmann to realize the formal intentions of the program and Frauke Bergmann for her patience and organisation skills. Most of the interesting collaboration and scientific discussion born in this framework were done with Marta Kocun about membrane stiffness, Sai Li about bending and curvature, and Plamen Dobrev about atomistic simulations and pca.

Aside from my program and my founding project, Alessio Quaglino was really helpful to let me understand continuum models and Tina Ghelani for the biological background.

I've largely appreciated the support of Prof. de Groot and his suggestions during the thesis committee meetings and the interesting discussions about synthetic membranes with Dr. Iwan Schaap and Prof. Jörg Enderlein.

It was particular illuminating to discuss during conferences and visits with Prof. Julian Shillcock (nanoparticle modeling), Prof. Michael Maskos (nanoparticle structure), Prof. David Morse (simulation techniques), Prof. Mark Sansom (protein induced modification), Prof. Michael Kozlov (energy contributions in bilayer membranes), and Dr Andrea Grafmüller (vesicle fusion). 
All the simulations run were performed on the clusters of the GWDG and HLRN.

I thank Alessandro Ferrantini and Prof. Enrico Carlon to have hosted me in the theoretical physics group of the university of Leuven.

I take this occasion to personally thank Alessio, Andrea, Daniela, David, Chris, Iliana, Ilaria, Marta, Mirko, Tina, and Vesna to have shared this scientific life and for their personal support. They have always been particularly careful to understand the particular situations and to give me a practical and priceless help. Their support kept me strong and motivated during this years and I was impressed by the intense friendships they have shown to me.

Apart from the scientific background I credit the moral support I always got from my WG during many years: Fabian, Jana, Jan Paul, Per, Marlene, Sarah, Axel, Lena, Theda, Britta, Chiara, Niko, KathaI, Patrick, KathaII, Moritz, Federico, Susi, and Ani.

Thanks to the people with whom, sharing a common interest in languages, we have found an important key to understand and appreciate cultural differences: Janine, Katja, Nina, and Tatiana.

Thanks to all the people of the water-polo and rugby teams, without the trainings with them my back would not have been able to bear the pain caused by being seated so many hours at day.

Thanks to Ahmed, Alfredo, Ana Paula, Andonia, Anja, Anna, Ciro, Debora, Derya, Eireann, Elena, Elisa, Emin, Ingrid, Kristina, Levke, Mario, Matthieu, Nadja, Nan, Nele, Samir, Sanaz, Vincenzo, YinYin, XiaoPeng for the kindness, the interesting discussions, and human warm.

Thanks a lot to Emily, Eva, Fred, Jan and Osamu for the shared interest in creating and playing music.

Thanks to my family for having always supported my decision, for having always let me feel home once back, and for not having stressed too much my lack of contacts.

Thanks to Caterina who has proven me that distances are relative.

Thanks to Maria for the deep understanding and communications.

Thanks to Ivan who have accompanied me playing the soundtrack of these years and donated me my first "concert". 


\section{list of publications}

2012

Transition path from two apposed membranes to a stalk obtained by a combination of particle simulations and string method M. Müller, Y.G. Smirnova, G. Marelli, M. Fuhrmans, and A.C. Shi, Phys. Rev. Lett. 108, 228103 (2012)

2012 Line tension controlled mechanism for influenza fusion H.J. Risselada, G. Marelli, M. Fuhrmans, Y. Smirnova, H. Grubmüller, S.J. Marrink, and M. Müller, PLoS ONE 7 e38302 (2012). 


\section{curriculum vitae}

personal data

$\begin{array}{ll}\text { name: } & \text { Giovanni Marelli } \\ \text { born: } & \text { 14/08/1983 Desenzano del Garda } \\ \text { address: } & \text { Kreuzbergring } 2137075 \text { Göttingen } \\ \text { nationality: } & \text { Italian } \\ & \\ \text { education } & \\ 1997 / 2002: & \text { Liceo Enrico Fermi, Salò } \\ 10 / 2002 & \text { Study of physics at the Università degli studi di Padova } \\ 12 / 2006 & \text { Bachelor in physics } \\ 03 / 2009 & \text { Master in physics } \\ 06 / 2009 & \text { PhD student at the Georg August Universität Göttingen }\end{array}$




\section{abstract}

Solvent-free soft coarse-grained models are particularly appropriate to investigate collective phenomena in lipid membranes. In this work we exploit such a model to show how modifying a few model parameters we can control the bending rigidity of the membrane, the hydration repulsion, and the macroscopic phases of self-assembled structures. Further, we investigate the lipid mediated interactions between fusion objects: transmembrane proteins, pores and stalks. The presence of such defects induces a perturbation in the shape of the membrane and in the conformations of lipids. The modifications induced by single defects superimpose and for some defect interaction (peptide-peptide, pore-peptide) we identify the equilibrium distance between these objects. The prediction of the results of the simulations are compared with the numerical solution of a continuum model parametrized from the analysis of the simulation snapshots.

The presence of transmembrane proteins with a large hydrophobic mismatch weakens the membrane over the direct interaction range decreasing slightly the membrane thickness. This involves the lowering of the line tension of the pore and for a particular number and spatial arrangement of proteins the line tension of the pore is negative and the pore is stable in tensionless membranes. Another agent that influences the line tension of the pore is oil (short hydrophobic chains). The oil has a configuration space larger than the one of the lipids and partitions to relax the frustration of the lipids at the interfaces and increases the line tension of the pore (the membrane is more resistant under lateral tension).

The model parameters have a large influence on the equilibrium properties of a stalk and we study the characteristic sizes of stalks depending on the hydration between two opposed bilayers and compare the results to other simulation models and experimental data. We show how hydration and lateral tension influence bilayer repulsion and how the combined effect of both contributions leads to membrane fusion. 


\section{Minimale Modelle für Lipidmembranen: lokale Änderung um Fusionsobjekte.}

\section{Zusammenfassung}

Lösungmittelfreie, weiche und vergröberte Modelle sind besonders geeignet um kollektive Phänomene von Lipidmembranen zu untersuchen. In dieser Arbeit werten wir solche Modelle aus, um zu zeigen wie wir, nach der Änderung von einigen Parametern, die Biegesteifheit, die Hydrationabstoßung und die makroskopischen Phasen von selbstassemblierten Strukturen steuern können.

Danach, untersuchen wir die lipidvermittelten Wechselwirkungen zwischen Fusionobjekten: transmembrane Proteine, Poren und Stalks. Die Anwesenheit von solchen

Störungsstellen schafft eine Änderung in der Lipidanpassung. Die Änderung wird durch einzelne Störungstellen erzeugt und überlappt in vielfältigen Störungstelleanordnungen. Wir bestimmen den Gleichgewichtsabstand zwischen diesen Objekten. Die Voraussagen dieser Simulationen werden mit der numerischen Lösung von kontinuierlichen Modellen verglichen, wobei das Modell durch die Analyse von Simulationschnapschüssen parametrisiert wurde.

Transmembrane Proteine mit großer hydrophober Unausgeglichenheit schwächen die Membran über den Wechselwirkungbereich hinaus und verringern leicht die Membrandicke. Das verursacht eine Abnahme der Porenlinienspannung, für eine bestimmte Anzahl und räumliche Anordnung von Proteinen wird die Porenlinienspannung negativ udn führt zu stabilen Poren in spannunglosen Membranen. Die Porenlinienspannung wird auch durch Öl (kurze hydrophobe Ketten) beeinflusst. Das Öl hat einen großeren Konfigurationraum als bei Lipiden und verteilt sich um die Frustration der Lipide an der Grenzfläche zu entspannen und um die Porenlinienspannung zu erhöhen (die Membran ist resistenter gegenüber lateralen Spannung).

Die Modellparameter haben einen großen Einfluss auf die Gleichgewichtseigenschaften eines Stalks. Wir untersuchen die charakteristischen Großen, die von der Hydration gegenseitiger Doppelschichten abhängig sind. Wir vergleichen die Ergebnisse von verschiedenen Simulationmodellen und experimentellen Daten. Wir zeigen wie die Doppelschichtabstoßung von Hydration und lateraler Spannung abhängig ist, und wie die kombinierten Effekte zur Membranfusion führen. 